\title{
Development of Nano-crystalline Doped-Ceramic Enabled Fiber Sensors for High Temperature In-Situ Monitoring of Fossil Fuel Gases
}

Final Report, March 2012

Prepared for:

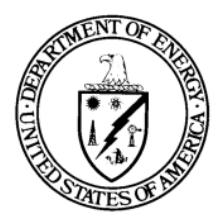

The United States Department of Energy National Energy Technology Laboratory

Under:

Contact No. DE-FC26-05NT42439

DOE Project Manager:

Susan Maley

Principal Investigators:

Prof. Hai Xiao, Department of Electrical and Computer Engineering,

Missouri University of Science and Technology, 573-342-6887, xiaoha@mst.edu

Prof. Junhang Dong, Department of Chemical and Materials Engineering

University of Cincinnati, 513-556-3992, junhang.dong@uc.edu

Prof. Jerry Lin, Department of Chemical and Materials Engineering

Arizona State University, 480-965-7769, jerry.lin@asu.edu

Prof. Van Romero, VP. for Research and Economic Development

New Mexico Institute of Mining and Technology, 505-835-5646,

vromero@admin.nmt.edu 
(This page intentionally left blank) 


\section{DISCLAIMER*}

This report was prepared as an account of work sponsored by an agency of the United States Government. Neither the United States Government nor any agency thereof, nor any of their employees, makes any warranty, express or implied, or assumes any legal liability or responsibility for the accuracy, completeness, or usefulness of any information, apparatus, product, or process disclosed, or represents that its use would not infringe privately owned rights. Reference herein to any specific commercial product, process, or service by trade name, trademark, manufacturer, or otherwise does not necessarily constitute or imply its endorsement, recommendation, or favoring by the United States Government or any agency thereof. The views and opinions of authors expressed herein do not necessarily state or reflect those of the United States Government or any agency thereof. 


\section{TABLE OF CONTENTS}

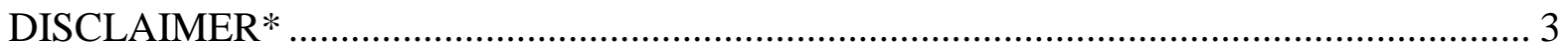

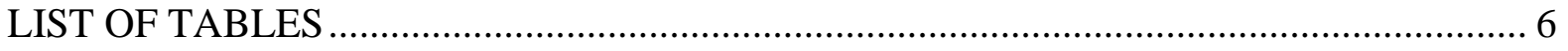

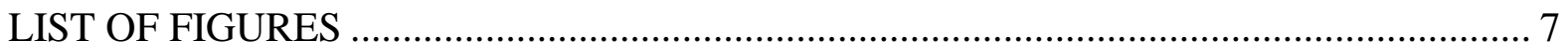

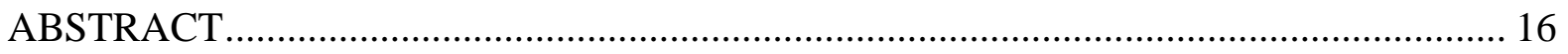

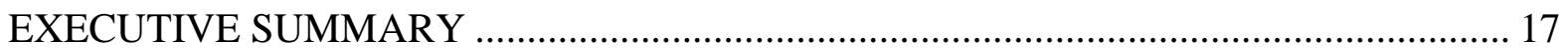

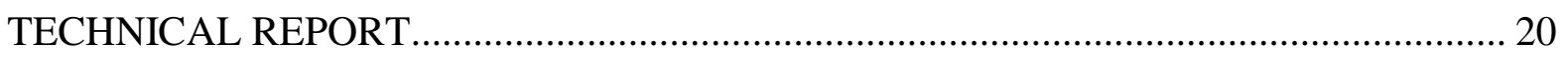

1. Research on Fiber Device Development............................................................... 20

1.1 Design and fabrication of evanescent wave attenuation (EWA) fiber device ......... 21

1.2 Design and fabrication of thermal LPFG fiber device .................................... 25

1.3 Fiber Inline Core-Cladding-Mode Mach-Zehnder Interferometer Fabricated by Two-

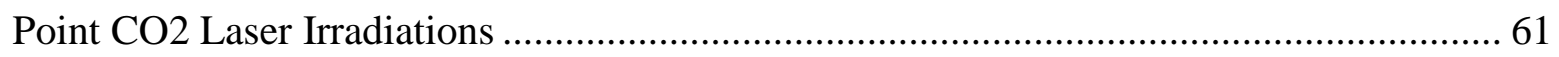

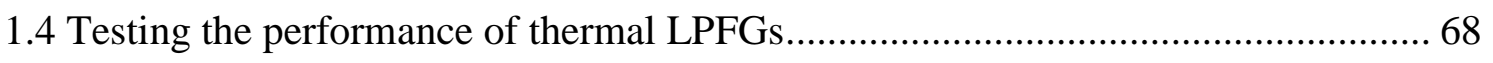

1.5 Fiber Ring Laser Interrogated Zeolite Coated SMS Structure for Trace Chemical

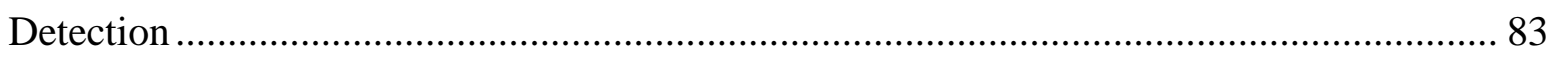

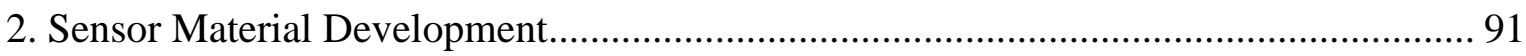

2.1 Synthesis and Characterization of proton-conducting Ceramics ........................... 93

2.2 Synthesis of nano-crystalline SDC/YSZ heterophase thin films on flat substrates . 96

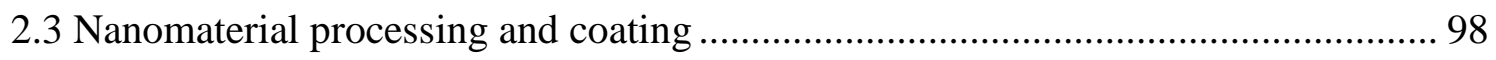

2.4 Pd thin film coating on optical fiber by sputtering deposition............................ 105

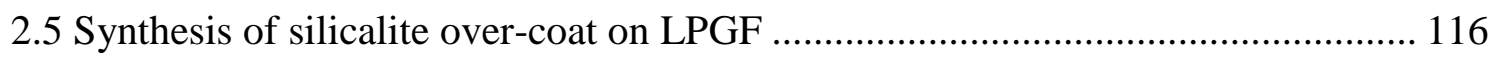


2.6 $\mathrm{Tb}$ doped $\mathrm{SrCeO}_{3}(\mathrm{SCTb})$ Hydrogen sensing material Development and

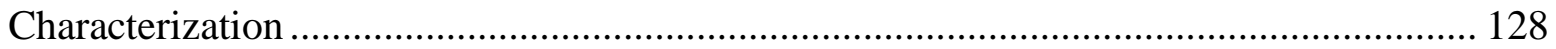

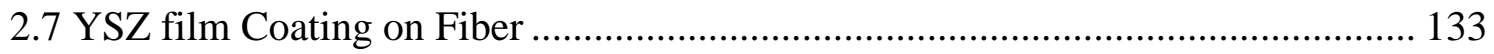

2.8 Material selection and development for gas Sensing ..................................... 135

2.9 Investigation of Nano-crystalline Copper-Doped Zirconia Thin Films for Optical Sensing of Carbon Monoxide at High Temperature ................................................... 139

2.10 Synthesis and Characterization of CDZ film ............................................. 141

3. Sensor Tests and Technology Demonstration...................................................... 144

3.1 Instrumentation and sensor testing of gas sensors ..................................... 144

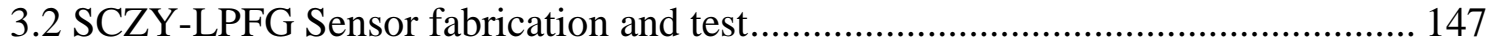

3.3 High temperature optical sensing with the CDZ-LPFG ................................. 154

3.4 Sensor performance enhancement by zeolite overcoate ................................. 176

3.5 Sensor installation and testing in an experimental gasifier............................. 181

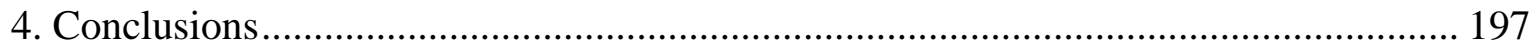

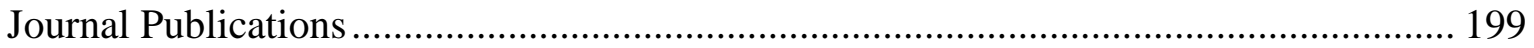

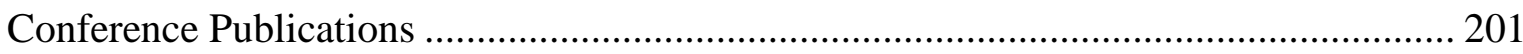

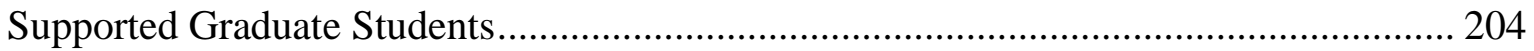




\section{LIST OF TABLES}

Table 1. Composition analysis on fiber side surface sputtered at 15mAmp ..................... 108

Table 2. Composition analysis on fiber end sputtered at $15 \mathrm{mAmp}$................................ 108

Table 3 Estimated grain size of $\mathrm{Pd}-\mathrm{Cu}$ annealed under different conditions ..................... 112

Table 4. Comparison of refractive index of Nano - crystalline $\mathrm{Pd}$ and bulk $\mathrm{Pd}$................ 115

Table $5 *$ Temperature testing using the ceramic adhesive ....................................... 183

Table 6 Pressure testing using the ceramic adhesive ................................................. 184

Table 7 Temperature testing using regular $\mathrm{Sn}-\mathrm{Pb}$ solder ........................................... 184

Table 8 Pressure testing using regular Sn-Pb solder ................................................. 185

Table 9 Gasification conditions for two separate sensor tests ..................................... 193

Table 10 Comparison of $\mathrm{H}_{2}$ composition by fiber sensor and $\mathrm{GC}$ analysis $(\mathrm{T}=500 \mathrm{oC}$ and $\mathrm{P}=$ 27.7 Psi) 194 


\section{LIST OF FIGURES}

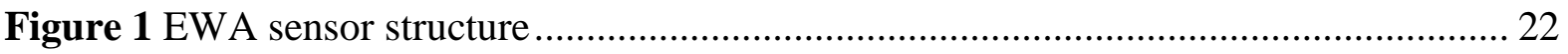

Figure 2 Fiber diameter as a function of etching time............................................ 23

Figure 3 Measurement of fiber diameter using an optical surface profiler........................ 23

Figure 4 Photo of the $\mathrm{CO}_{2}$ laser based fiber modification station. .................................. 24

Figure 5 Microscopic image of an optical fiber modified by $\mathrm{CO}_{2}$ laser .......................... 24

Figure 6 Microscopic image of an optical fiber etched after $\mathrm{CO}_{2}$ laser irradiation.............. 24

Figure 7 Schematic illustration of the CO2 laser based LPFG fabrication system............... 31

Figure 8 Photograph of the $\mathrm{CO}_{2}$ laser based LPFG fabrication system........................... 31

Figure 9 Thermal LPFG, (a) microimage of a fabricated thermal LPFG, (b) typical transmission spectrum of a thermal LPFG....................................................................... 33

Figure 10 Transmission spectrum of the typical LPFG fabricated in our lab.................... 34

Figure 11 LPFG resonance wavelength as a function of the grating period........................ 35

Figure 12 Transmission spectra of the LPFGs with different period corresponding resonance

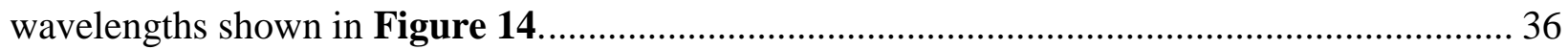

Figure 13 Periodic CO2 laser irradiation induced refractive index modulation in an optical fiber. 38

Figure 14 Fiber Fabry-Perot interferometer for measurement of refractive index modulation 40

Figure 15 Block diagram of CO2 laser based LPFG fabrication system 43

Figure 16 Optical length changes of fiber segment caused by $\mathrm{CO} 2$ laser irradiations with the power of $8 \mathrm{~W}$ and exposure time of $125 \mathrm{~ms}$; (a) interference spectra; (b) change in OL as a 
function of the number of laser exposures.

Figure $17 \mathrm{CO} 2$ laser irradiation induced fiber refractive index changes at various laser exposure times (50,75, 100 and $125 \mathrm{~ms}$, respectively); (a) OL change as a function of the number of laser exposures; (b) $\Delta$ nmax as a function of laser exposure time. 47

Figure 18 Comparison between the measured and simulated transmission LPFG spectra. Fiber parameters (Corning SMF-28e) from datasheet were used in the simulations. Inset table: detailed comparisons between measured and simulated resonant wavelengths and peak strengths.

Figure 19 Comparison between the simulated (with slightly modified core-cladding index contrast) and measured transmission LPFG spectra. Inset table: detailed comparisons between measured and simulated resonant wavelengths and peak strengths. 49

Figure 20 Phase matching curve for studied LP10 mode in corning SMF during various surrounding refractive index environments. 55

Figure 21 The grating spectra evolution of LP010 mode in respond to surrounding refractive index change (1.27 1.38) (top); the transmission power (TP LPFG) curve at different RI liquids (bottom left); the transmission power linear part at spectral RI range (bottom right) 57

Figure 22 The grating spectra evolution of LP010 mode in respond to different concentration of sucrose solution (0-30\%) in experiment with grating period $\Lambda=230 \mu \mathrm{m}$ (top); the transmission power curve at different RI liquids (bottom left); and the transmission power linear trend at specific range (bottom right). 59

Figure 23 Fiber inline core-cladding-mode MZI fabricated by 2-point CO2 laser irradiation: (a) schematic diagram; (b) microscopic image of laser induced micronotch; (c) illustration of the interference spectrum. 61 
Figure 24 Transmission spectra of fiber inline core-cladding-mode MZIs with different

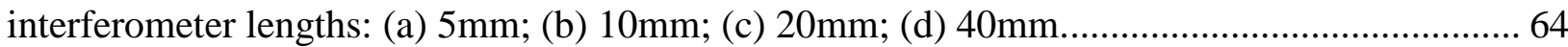

Figure 25 Temperature response of the fiber inline MZ-CCMI with a length of 5mm fabricated by 2-point $\mathrm{CO} 2$ laser irradiation. 65

Figure 26 Wavelength shift as a function of refractive index in MZ-CCMI and LPFGs with LP04 and LP05 cladding modes (Inset: transmission spectrum of LPFG with LP05 cladding mode). 66

Figure 27 LPFG transmission spectrum at various ambient temperatures. The LPFG had a period of $500 \mu \mathrm{m}$ and was fabricated using Corning SMF-28 single mode fibers. 70

Figure 28 Shifts in the LPFG resonance wavelength as a function of temperature. 70

Figure 29 LPFG transmission spectrum in air, water, and isopropanol. The LPFG has a period of $515 \mu \mathrm{m}$ and was fabricated using Corning SMF-28 single mode fibers. 71

Figure 30 Shift of the resonance wavelength caused by bending. The LPFG has a period of $515 \mu \mathrm{m}$ and was fabricated using Corning SMF-28 single mode fibers. 73

Figure 31 Shift of the LPFG transmission spectrum in response to annealing at $650^{\circ} \mathrm{C}$ for 8 hours. The LPFG had a period of $500 \mu \mathrm{m}$ and was fabricated using Corning SMF-28 single mode fibers. 74

Figure 32 Drift of the LPFG resonance wavelength caused by annealing at $650^{\circ} \mathrm{C}$. 75

Figure 33 Shift of the LPFG transmission spectrum in response to annealing at $700^{\circ} \mathrm{C}$ for 30 hours. The LPFG had a period of $515 \mu \mathrm{m}$ and was fabricated using SMF-28 single mode fibers. 76

Figure $34 \mathrm{LPFG}$ resonance wavelength as a function of annealing time at $700^{\circ} \mathrm{C}$. 76

Figure 35 LPFG peak transmission as a function of annealing time at $700^{\circ} \mathrm{C}$. 77

Figure 36 LPFG spectrum before and after 200 hours aging at $550^{\circ} \mathrm{C}$ 78 
Figure $37 \mathrm{LPFG}$ resonance wavelength change as a function of time at $550^{\circ} \mathrm{C}$.

Figure 38 LPFG spectrum at 0 hour and 200 hour at $550^{\circ} \mathrm{C}$ after annealing at $800^{\circ} \mathrm{C}$ for 1 hour 80

Figure $39 \mathrm{LPFG}$ resonance wavelength drift at $550^{\circ} \mathrm{C}$ after annealing at $800^{\circ} \mathrm{C}$ for 1 hour. 81

Figure 40 Drift in resonance wavelength of LPFG within 60 days in $550^{\circ} \mathrm{C}$. Inserts: transmission spectra of LPFG at the beginning and ending of the test, respectively. 82

Figure 41 Drift in peak strength of LPFG within 60 days in $550^{\circ} \mathrm{C}$. 82

Figure 42 Zeolite coated SMS fiber structure. (a) Device schematic, (b) and (c) SEM images of zeolite-coated optical fiber, (d) Transmission spectrum of the zeolite-coated SMS structure. 85

Figure 43 Experimental setup for sensor evaluation and schematic of fiber ring laser..... 87

Figure 44 Lasing spectra of the zeolite-coated SMS structure at various ethanol concentrations 88

Figure 45 Center wavelength of the zeolite-coated SMS fiber ring laser as a function of ethanol vapor concentration. 89

Figure 46. Electrical conductivity of synthesized perovskite type ceramics under He and air.

Figure 47 XRD patterns. Lines $\mathrm{a}, \mathrm{b}$ and $\mathrm{c}$ are YSZ, SDC and YSZ/SDC solid solution pellets (Ref. 8), respectively. $\mathrm{d}$ and e are films synthesized with untreated SDC: $\mathrm{d}$ - fired at $300^{\circ} \mathrm{C}$ and e - fired at $450^{\circ} \mathrm{C}$ (solid solution). f, $\mathrm{g}$ and $\mathrm{h}$ are films with SDC pretreated at $1200^{\circ} \mathrm{C}$ : f - dried at $80^{\circ} \mathrm{C}, \mathrm{g}$ - fired at $650^{\circ} \mathrm{C}$, and $\mathrm{h}$ - fired at $850^{\circ} \mathrm{C}$ 100

Figure 48 AFM image of the surface of the YSZ800/SDC heterophase film annealed at $750^{\circ} \mathrm{C}$ for 5 hours. 101

Figure 49. SEM image of the cross-section of the SDC800/YSZ heterophase film..... 101 
Figure 50. TEM observations of a SDC/YSZ hetero-phase film cross section. (a) TEM image; (b) STEM Z-contrast mapping (contrast inverted for better observations). 102

Figure 51. Electrical Conductivity as a function of temperature. $\bigcirc$ SDC800/YSZ heterophase film, $\times$ SDC1000/YSZ, SDC1200/YSZ hetero-phase film, $\diamond$ YSZ nano-film (this work), YSZ nano-film (grain size $20 \mathrm{~nm}$ ) (Ref. 10), - SDC nano-film, $\square$ SDC pellet (Ref. 8), + YSZ pellet. 103

Figure 52. Electrical Conductivity as a function of SDC volume fraction at different temperatures. $\left(\boldsymbol{\nabla} 450^{\circ} \mathrm{C}, \mathbf{\Delta} 550^{\circ} \mathrm{C}, 650^{\circ} \mathrm{C}, \bullet 750^{\circ} \mathrm{C}\right)$ 104

Figure 53. SEM images of Pd coated on optical fiber at the sputtering power of $15 \mathrm{mAmp}$. (a) and (b) side surface coating at different fiber sections, (c) fiber endface coating, and (d) Pt particles on fiber surface. 106

Figure 54. SEM image of $\mathrm{Pd} / \mathrm{Si}$ fiber interface. 107

Figure 55. SEM images of Pd coated on optical fiber at the sputtering power of 9mAmp. 109

Figure 56. Microscopic optical images of pd-coated optical fiber by electroless plating ... 110

Figure 57. SEM image of Pd particles on optical fiber by electroless plating 111

Figure 58. XRD patterns of $\mathrm{Pd}(58 \%)-\mathrm{Cu}$ alloy before and after annealing..... 112

Figure 59. SEM images of $\mathrm{Pd}-\mathrm{Cu}$ alloy (a) without treatment, (b) annealed at 500oC for 6 hours, (c) 12 hours and (d) annealed at $800 \mathrm{C}$ for 18 hours. 113

Figure 60. $\mathrm{n}$, $\mathrm{k}$ of Pd thin film deposited on alumina bar measured by ellipsometer 114

Figure 61. SEM images of the silicalite coated LPGF (left: surface; right: cross-section). 116

Figure 62. Monitoring of the zeolite overcoat synthesis process 117

Figure 63. Results of the zeolite LPGF sensor for detection of trace chemical vapors...... 118

Figure 64. Correlations between the sensor output signals and the toluene concentration . 119 
Figure $65 \mathrm{SEM}$ pictures of the $\mathrm{ZrO}_{2} / \mathrm{CuO}-\mathrm{LPFG}$ sensor

Figure 66 Resonant wavelength as a function of $\mathrm{CO}$ concenration in $\mathrm{CO}_{2}$ at $550^{\circ} \mathrm{C}$ and 1 atm.

Figure 67 SEM images of the MFI-LPFG sensor 122

Figure $68 \mathrm{MFI}-\mathrm{LPFG}$ sensor response to $\mathrm{H} 2$ concentration in $\mathrm{N}_{2}$ at $400^{\circ} \mathrm{C}$. 123

Figure 69 Sensor response to gas switching 126

Figure 70 Resonance wavelength of SCTb-LPFGs as a function of time at $600^{\circ} \mathrm{C}$ under different gas environments 128

Figure 71 Resonance wavelength of SCTb-LPFGs as a function of time under various $\mathrm{H}_{2}$ partial pressure at $600{ }^{\circ} \mathrm{C}$.

Figure 72 Resonance wavelength increase rate versus $\mathrm{H}_{2}$ partial pressure at $600{ }^{\circ} \mathrm{C}$ 129

Figure 73 Refractive index of $\mathrm{SCTb}$ film with and without $\mathrm{H}_{2}$ treatment. 130

Figure $74 \mathrm{XRD}$ pattern of as synthesized SCTb powder and one after treated in $4 \% \mathrm{H}_{2}$ at $600^{\circ} \mathrm{C}$ for 5 hours. 131

Figure 75 XPS spectra of as synthesized SCTb powder and after treated in $4 \% \mathrm{H}_{2}$ at $600^{\circ} \mathrm{C}$ for 5 hours 132

Figure 76 Optical fiber coated with YSZ thin film using YSZ sol 133

Figure 77 Optical fiber coated with YSZ thin film using YSZ suspension ..... 134

Figure $78 \mathrm{SCZY}$ coated LPFG in response to $\mathrm{H}_{2}$ concentration variations. 145

Figure 79 SEM images of single-time coated SCTb-polymeric thin film: (a) pre-fire; (b) post-fire. 148

Figure 80 SEM images of the 5-time coated SCTb-LPFG sensor showing $\sim 400 \mathrm{~nm}$ SCTb nano crystalline thin film on the LPFG. 149 
Figure 81 Results of SCTb-LPFG sensor tests in H2/N2, CO/N2 and CO2/N2 binary gas mixtures at 500oC and 1 atm: (a) Stabilized transmission spectra in $\mathrm{H} 2 / \mathrm{N} 2$ with various $\mathrm{H} 2$ concentrations; (b) $\lambda \mathrm{R}$ shift $(\Delta \lambda \mathrm{R})$ as a function of $\mathrm{H} 2, \mathrm{CO}$ and $\mathrm{CO}_{2}$ concentrations. 150

Figure 82 SEM images of pervoskite thin films on optical substrates: (a) SCTb thin film; (b) SCTm thin film; (c) SCZY thin film. 152

Figure 83 SCZY-LPFG sensor response $\left(\mathrm{Dl}_{\mathrm{R}}\right)$ to bulk $\mathrm{H}_{2}$ in $\mathrm{N}_{2}$ at different temperature (left) and the cross-section SEM image of the sensor tested. 154

Figure 84 Schematic diagram of the high temperature optical testing system 155

Figure 85 FTIR spectra of ethyl glycol (EG), poly ethyl glycol (PEG) metal-chaleted PEG (Metal-PEG). 157

Figure 86 SEM pictures of (a) cross-section of a single time coated precursor film after drying, (b) cross-section of the four-time coated CDZ film on fiber, and (c) surface of the CDZ film on fiber with insert showing TEM picture of CDZ particles after $96 \mathrm{~h}$ of annealing at $550^{\circ} \mathrm{C}$.

Figure 87 XRD pattern of the CDZ particles together with standard patterns of $\mathrm{T}-\mathrm{ZrO} 2, \mathrm{M}$ $\mathrm{ZrO} 2, \mathrm{CuO}$ and $\mathrm{Cu}_{2} \mathrm{O}$ 160

Figure 88 The transmission spectra of the LPFG before and after coating CDZ. 161

Figure 89 The CDZ-LPFG response to switching between air and 1,000 ppmv CO in N2 at 550oC: (a) transmission spectra in the two gases and (b) the single wavelength $(\lambda=1610 \mathrm{~nm})$ temporal responses to gas switching (arrows indicating gas switching points). 163

Figure 90 (a) The CDZ-LPFG single wavelength $(\lambda=1576 \mathrm{~nm})$ response to switch between air and 1vol\% $\mathrm{CO}$ in $\mathrm{N} 2$ at $550 \mathrm{oC}$ (arrows indicating points of gas switch) and (b) the transmission spectra at different times in contact with 1 vol\% $\mathrm{CO}$. 166 
Figure $91 \mathrm{XRD}$ spectra of the CDZ particles after being treated at $550 \mathrm{oC}$ in: a - fresh sample; $\mathrm{b}-2 \mathrm{~h}$ in 400 ppmv CO; $-2 \mathrm{~h}$ in air; $\mathrm{d}-$ after four cycles of switching between air and 400 ppmv CO with a final step of $10 \mathrm{~h}$ in 400 ppmv CO.

Figure 92 XRD spectra of the CDZ particles after being treated at 550oC: a - fresh sample, b - in $1 \mathrm{vol} \% \mathrm{CO}$ for $2 \mathrm{~h}, \mathrm{c}$ - after three cycles of switching between air and 1vol\% CO ending with $2 \mathrm{~h}$ in 1 vol\% $\mathrm{CO}, \mathrm{d}-$ after $2 \mathrm{~h}$ in air. 168

Figure 93 TEM images and electron diffraction patterns of the $\mathrm{Cu}$-doped $\mathrm{CDZ}$ nanoparticles: (a) and (b) - fresh CDZ particle; (c) and (d) after reduction in 1vol\% CO for $10 \mathrm{~h}$. 170

Figure $94 \mathrm{Zr}$ and $\mathrm{Cu}$ content distributions measured by EDX line scanning for (a) fresh CDZ nanoparticle and (b) CDZ nanoparticles after $3 \mathrm{~h}$ of treatment in $1 \mathrm{vol} \% \mathrm{CO}$ at $550 \mathrm{oC}$ (inserts are TEM images showing the scanning lines position). 172

Figure 95 CDZ-LPFG transmission spectra gases of different $\mathrm{CO}$ concentrations (a) and sensor output $\Delta \lambda \mathrm{R}$ as a function of $\mathrm{CO}$ concentration at $550^{\circ} \mathrm{C}$ (b). 174

Figure 96 Schematic showing the LPFG sensor placement arrangement 186

Figure 97 Chamber installation and testing, installed chamber is shown in a red circle ..... 187

Figure 98 Chamber installation and testing, installed chamber is shown in a red circle.... 188

Figure 99 Picture of field testing. 189

Figure 100 Pictures for the actual gasifier equipped with the sensor test unit. 190

Figure 101 Screenshots of the gasifer (top) and sensor (bottom) computer operating programs. 192

Figure 102 Transmission spectra shifts for the temperature sensor (LPFG) and the $\mathrm{H}_{2}$ sensor (SCZY-LPFG) at various stages of gasifier operation (in Test No. 2) 195

Figure 103 Online measured SCZY-LPFG resonant wavelength $\left(\lambda_{R}\right)$ and temperature as 
function of time during gasification:.

Figure 104 Wavelength shifts $\left(\Delta \lambda_{R}\right)$ of the SCZY-LPFG caused by temperature change and $\mathrm{H}_{2}$ concentration (partial pressure) change as function of operation time (online monitoring). 196 Figure 105 Hydrogen content monitored during gasification (partial pressure vs. time; and mole fraction vs. time) 197 


\begin{abstract}
This is a final technical report for the first project year from July 1, 2005 to Jan 31, 2012 for DoE/NETL funded project "DE-FC26-05NT42439: Development of Nanocrystalline DopedCeramic Enabled Fiber Sensors for High Temperature In-Situ Monitoring of Fossil Fuel Gases.” This report summarizes the technical progresses and achievements towards the development of novel nanocrystalline doped ceramic material-enabled optical fiber sensors for in situ and real time monitoring the gas composition of flue or hot gas streams involved in fossil-fuel based power generation and hydrogen production.
\end{abstract}




\section{EXECUTIVE SUMMARY}

This final report summarizes the technical progresses and achievements made towards the development of novel nano-crystalline doped ceramic material-enabled optical fiber sensors for in situ and real time monitoring the gas composition of flue or hot gas streams involved in fossilfuel based power generation and hydrogen production.

Research was conducted in the four areas including 1) optical fiber devices development, 2) sensor material development, synthesis and characterization, and 3) sensing testing. The technical progresses and achievements are summarized as follows. All the milestones set for the project has been successfully met.

The fiber device research was focused on the following: 1) design and fabrication of evanescent wave attenuation (EWA) fiber device, 2) design and fabrication of thermal long period grating fiber devices, 3) design and fabrication of fiber inline core-cladding mode interferometer, and 4) testing the performance of thermal long period fiber grating (LPFG). The objective is to design and develop a suitable fiber optic platform that can optimally work with the nanofilm materials to perform highly sensitive gas detection in harsh environments. Three methods have been investigated to fabricate the EWA fiber device, including chemical etching to remove the silica cladding, $\mathrm{CO}_{2}$ laser radiation to expend the core region, and $\mathrm{CO}_{2}$ laser mark assisted chemical etching. We found that $\mathrm{CO}_{2}$ laser irradiation and $\mathrm{CO}_{2}$ laser-mark assisted etching were the most promising methods to fabricate the proposed EWA fiber devices. Thermal LPFGs have also been successfully fabricated using the $\mathrm{CO}_{2}$ irradiation based methods. A rigorous theoretic model was developed to simulate the transmission spectrum with/without coating. The automated fabrication station for LPFG is fully functioning with real-time 
monitoring system. The index modulation was measured by a unique methodology developed based on an interferometer. LPFG can be well modeled and optimized by inputting the measured index modulation into the theoretic model. High order mode LPFG refractive index sensor based on intensity measurement was also developed, which allows resonance intensity based measurement instead of conventional used resonant peak shift based measurement for a LPFG. Multiplexing concept was developed, which allows LPFG multiplexing up to 10. Fiber inline core-cladding mode interferometer has been developed using the same fabrication stage as the LPFG. The fiber inline core-cladding mode interferometer uses the evanescent wave for detection, which has a similar sensitivity as LPFG. Our evaluations indicate that the thermal LPFG can survive high temperature conditions. In addition, we found that the annealing process drastically improved the thermal stability of LPFG devices.

The research in the area of material development was focused on four areas including 1) sensor material synthesis, identification, and characterization, 2) nanomaterial synthesis, processing and fiber coating, 3) optical fiber devices design, fabrication, and characterization, and 4) Laboratory and field testing the sensor devices. Appropriate nano-materials were identified, synthesized and integrated with the optical fibers for developing sensors for gas detection. The work was specifically focused towards $\mathrm{H}_{2}, \mathrm{CO}_{2}$, and $\mathrm{CO}$ gas detection.

The performance of the integrated sensors were tested and verified in laboratory simulated gas mixtures at high temperatures. The sensors have been tested in single-component as well as multi-component high temperature environments, showing high sensitivity $(\sim \mathrm{ppm})$, large dynamic range (ppm to $80 \%$ for hydrogen sensing), long lifetime (>60 days), and high operating temperature $\left(>500^{\circ} \mathrm{C}\right)$. The encouraging results lead to further planning the field tests.

Encouraged by the results in fundamental research, the research team has been tasked to 
move a step further into the applied research of testing the developed sensors in a university experimental gasifier. In this new task, we have conducted research in packaging of the sensors, modification of the gasifier for sensor installation, and development of instrumentation for onsite data logging. The developed sensors have been successfully survived the harsh conditions and operated for a long-period time. The $\mathrm{H}_{2}$ concentrations in the syngas obtained by the sensor and GC analyses are in a very good agreement. 


\section{TECHNICAL REPORT}

\section{Research on Fiber Device Development}

The fiber device research in this period was focused on the following: 1) design and fabrication of evanescent wave attenuation (EWA) fiber device, 2) design and fabrication of thermal long period grating fiber devices, 3) design and fabrication of thermal core-cladding mode interferometer, 4) Thermal stability test and optimization. The objective is to design and develop a suitable fiber optic platform that can optimally work with the nano-coating materials to perform highly sensitive gas detection in harsh environments. 


\subsection{Design and fabrication of evanescent wave attenuation (EWA) fiber device}

Evanescent wave (EW) is the standing wave formed at the fiber core and cladding interface, where a small portion of the propagating energy penetrates into the cladding region to a distance comparable with the wavelength of the guided light. If the evanescent field is exposed to the surrounding environment, the surrounding gas molecules will interact with the evanescent field, which changes the light propagating inside the fiber via frustrated total internal reflection (FTIR) or attenuated total internal reflection (ATIR). The FTIR is related to the change of the refractive index of the cladding while the ATIR is related to the change of the cladding absorption coefficient. Although these two mechanisms were investigated to make evanescent wave fiber gas sensors, the ATIR is more practical to work with the nanocrystalline coating. Therefore, our investigation has been mainly focused on the evanescent wave attenuation (EWA) sensor.

\subsubsection{EWA sensor design}

The design of EWA sensors was mainly focused on using multimode fibers. Multimode fibers have large core diameters. They have enough mechanical strength after the cladding is removed.

Figure 1 schematically illustrates the operating principle of the EWA sensor. The sensor is made by replacing the silica glass cladding of a multimode fiber with the nanocrystalline chemisober (doped ceramic) film. A thin layer of silicalite is overcoated to protect the chemisober film. In order to maintain the wave guiding property, the refractive index of the silicalite needs to be smaller than that of the fiber core. 


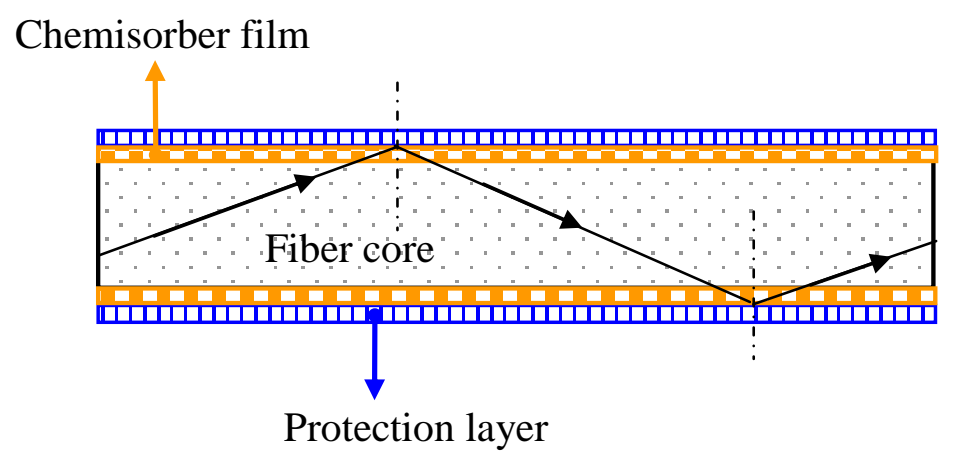

Figure 1 EWA sensor structure

When the light is guided through the sensing section, it is guided by the interface between the fiber core and the protection layer. At the interface between the fiber core and the chemisorber film, the light interacts effectively with the chemisorber film through the evanescent field. The unique microporous structure of the silicalite overcoating will allow the target gas molecules to pass through and contact the chemisober film, resulting in changes in its optical absorption (the imagery part of the refractive index). Therefore, the amount of target gas can be known by monitor the optical attenuation.

\subsubsection{EWA device fabrication}

Three methods have been investigated to fabricate the EWA sensor, which mainly involved developing methods to access the fiber evanescent field in a controlled fashion. These three methods include: chemical etching to remove the silica cladding, $\mathrm{CO}_{2}$ laser radiation to expend the core region, and $\mathrm{CO}_{2}$ laser mark assisted chemical etching.

Chemical etching: Optical fibers were etched using hydrofluoric (HF) acid to remove the cladding layer. The HF was $49 \%$ in concentration and purchased from Fisher Scientific. Five fibers were completely immersed into the solution and taken out from the solution one by one at a fixed time interval of one minute. The etched fibers were immediately rinsed using $5 \%$ sodium 
hydroxide $(\mathrm{NaOH})$ solution and further by deionized (DI) water.

After drying, the diameters of the etched fibers were measured using the optical profiler (Weeko, NT1000) as shown in Figure 3. To minimize the measurement error, a standard unetched fiber was used as reference. Figure 2 shows the fiber diameter as a function of the etching time. The average etching rate was calculated to be $2.1 \mu \mathrm{m} / \mathrm{min}$. This etching rate is slow enough for accurate diameter control.

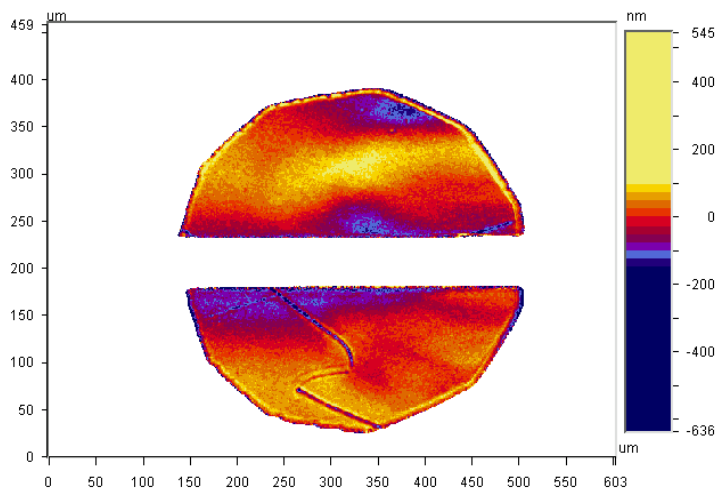

Figure 3 Measurement of fiber diameter using an optical surface profiler.

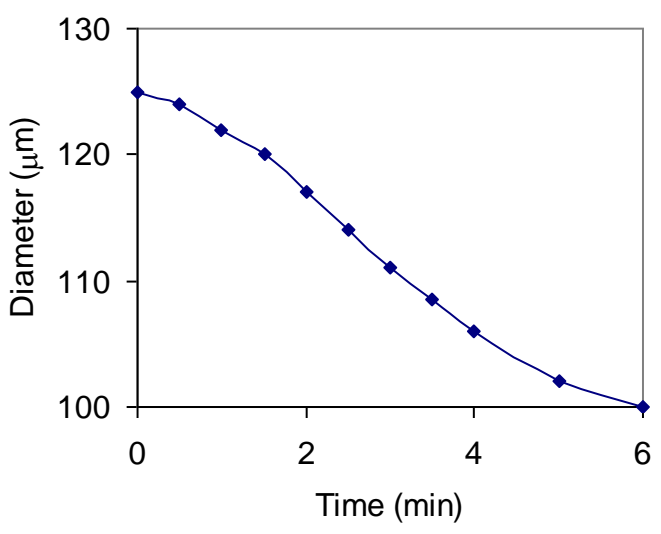

Figure 2 Fiber diameter as a function of etching time.

$\mathrm{CO}_{2}$ laser irradiation: An existing $\mathrm{CO}_{2}$ laser system was modified for fiber modification. As shown in Error! Reference source not found., the system included a pulsed $\mathrm{CO}_{2}$ laser (Universal Laser Systems, OEM-40), x-y-z stage systems, the fiber positioning mechanism, and a computer. Initial fiber modifications were tried using $\mathrm{CO}_{2}$ laser irradiation. Error! Reference source not found. shows the microscopic image of the modified fiber, where the modified section can be clearly identified. The initial results proved that $\mathrm{CO}_{2}$ could effectively change the refractive index profile of an optical fiber. Further experiments will be conducted to optimize the irradiation conditions to allow for a better control of the modification process. 


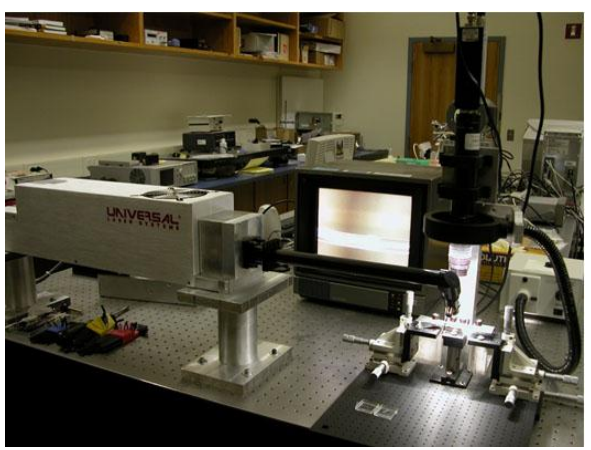

Figure 4 Photo of the $\mathrm{CO}_{2}$ laser based fiber modification station.

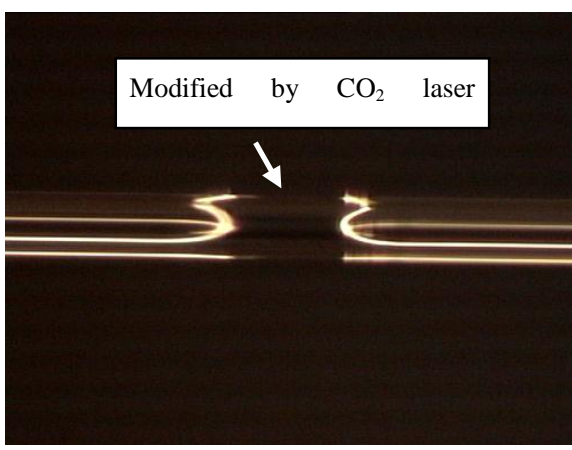

Figure 5 Microscopic image of an optical fiber modified by $\mathrm{CO}_{2}$ laser

$\mathrm{CO}_{2}$ laser-mark assisted fiber etching: Direct chemical etching has the disadvantage of nondirectional. To improve the etching directivity, we first used the $\mathrm{CO}_{2}$ laser irradiation to generate a mark on the optical fiber, where a higher stress was resulted from the thermal treatment. The laser marked fiber was then etched using HF solution. As shown in Error! Reference source not found., the laser-marked section has shown a much better directivity. The laser-mark assisted fiber etching thus provides an additional dimension to control the fiber device fabrication process.

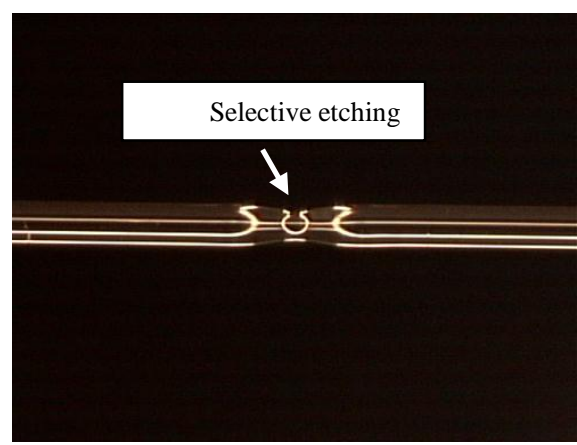

Figure 6 Microscopic image of an optical fiber etched after $\mathrm{CO}_{2}$ laser irradiation. 


\subsection{Design and fabrication of thermal LPFG fiber device}

A long-period fiber grating (LPFG) is a fiber device with induced periodic changes in the refractive index of optical fibers with a period many times higher than the light wavelength in the fiber. In a LPFG, there is one fundamental core mode and multiple cladding modes, which all propagate in the same (forward) direction. Long-period fiber gratings can couple light effectively the energy propagating in the fundamental core mode to the discrete cladding modes, resulting in the transmission spectrum of the fiber containing a series of attenuation bands centered at discrete wavelengths, which are called resonance wavelength. Each resonance wavelength corresponds to the coupling to a different cladding mode. The exact form of the spectrum, and the centre wavelengths of the attenuation bands, is sensitive to the period of the LPFG and the local environment: temperature, strain, bend radius and to the refractive index of the medium surrounding the fiber. Changes in these parameters can modify the phase matching conditions for coupling to the cladding modes, and results in significant peak position shifts.

LPFGs have attracted great interest in the optical telecommunication and sensor applications. Now the muti-cladding LPFGs with a film coated on the cladding area of the fiber grating has attracted much attention. In particular, if the coated film is gas sensitive, the LPFG can be used as gas sensors.

\subsubsection{LPFG Model}

For a long-period fiber grating, the phase-matching condition is

$$
\lambda=\left[n_{\text {eff }}{ }^{\text {core }}-n_{\text {op }}{ }^{\text {clad }}\right] \Lambda
$$

where $n_{\text {eff }}^{\text {core }}$ and $n_{o p}^{\text {clad }}$ are the respective core and cladding mode effective refractive indices, $\Lambda$ 
is the period of the grating and $\mathrm{p}$ is the order of the cladding mode.

The effective indices of the cladding modes are dependent upon the difference between the refractive index of the cladding and that of the medium surrounding the cladding. The central wavelengths of the attenuation bands thus show a dependence upon the refractive index of the cladding and the medium surrounding the cladding. Because the period of the grating is much larger than the resonance wavelength, LPFGs show sensitivity to change on the refractive index of the cladding, external medium or coating. This makes LPFGs useful as chemical sensors, because the fiber coating may be manufactured from an adsorptive material that absorbs any liquid or gas substance and effectively changes the ambient refractive index. If a grating is placed in a medium, the resulting shift in the resonance wavelengths due to the changes in ambient index can be used to determine the composition of the medium.

The interactions considered in this report occur mainly between the fundamental core mode (LP01 or HE11) and the cladding modes of a step-index fiber. Before we can apply the coupledmode theory, we must know how the light pregnant in code mode and cladding mode. In another word, we must know the all the necessary propagation constants. We assume that the fiber has a simple three-layer, step-index fiber geometry. With this assumption, we can readily calculate the fields of this structure and derive explicit expressions for the dispersion relations, the field profiles and intensity distributions, and the coupling coefficients. For the fundamental core mode, the linearly polarized (LP) approximation should be sufficient to describe a mode guided by the fiber core. We use this description to find the mode propagation constant. the dispersion relation that we solve to obtain the $\mathrm{LP}_{01}$ mode effective index is

$$
V \sqrt{1-b} \frac{J_{1}(V \sqrt{1-b})}{J_{0}(V \sqrt{1-b})}=V \sqrt{b} \frac{K_{1}(V \sqrt{b})}{K_{0}(V \sqrt{b})}
$$


where $\mathrm{J}$ is a Bessel function of the first kind, $\mathrm{K}$ is a modified Bessel function of the second kind, $V=(2 \pi / \lambda) a_{1} \sqrt{n_{1}^{2}-n_{2}^{2}}$ is the $\mathrm{V}$ number of the fiber at a wavelength $\lambda, \mathrm{b}$ is the normalized effective index, given by $b=\left(n_{\text {eff }}^{2}-n_{2}^{2}\right) / n_{1}^{2}-n_{2}^{2}, n_{1}$ and $n_{2}$ are the refractive index of the core and cladding, respectively. By solving the dispersion equation, we can get the effective refractive index of the fundamental core mode $\left(n_{2}<n_{e f f}^{c o}<n_{1}\right)$ and the pregnant constant. Then we can approximate the exact radial and azimuthal vector components of the $\mathrm{HE}_{11}$ mode fields in the core region of the fiber $(\mathrm{r}<\mathrm{a} 1)$ as

$$
\begin{aligned}
& E_{r}^{c o}=i A J_{0}\left(V \sqrt{1-b} r / a_{1}\right) \exp (i \phi) \exp [i(\beta z-\omega t)] \\
& E_{r}^{c o}=-A J_{0}\left(V \sqrt{1-b} r / a_{1}\right) \exp (i \phi) \exp [i(\beta z-\omega t)]
\end{aligned}
$$

where $A$ is the normalization constant. The cladding modes are somewhat more complicated than the core modes, since we may not neglect one of the interfaces. The dispersion equation of cladding modes for such a three-layer fiber have been given by Erdogan as following

$$
\zeta_{0}=\zeta_{0}^{\prime}
$$

where

$$
\zeta_{0}=\frac{1}{\sigma_{2}} \frac{u 2\left(J K+\frac{\sigma_{1} \sigma_{2} u_{21} u_{32}}{n_{2}^{2} a_{1} a_{2}}\right) p_{l}\left(a_{2}\right)-K q_{l}\left(a_{2}\right)+J r_{l}\left(a_{2}\right)-\frac{1}{u_{2}} s_{l}\left(a_{2}\right)}{-u_{2}\left(\frac{u_{32}}{n_{2}^{2} a_{2}} J-\frac{u_{21}}{n_{1}^{2} a_{1}} K\right) p_{l}\left(a_{2}\right)+\frac{u_{32}}{n_{1}^{2} a_{2}} q_{l}\left(a_{2}\right)+\frac{u_{21}}{n_{1}^{2} a_{1}} r_{l}\left(a_{2}\right)}
$$




$$
\zeta_{0}^{\prime}==\sigma_{1} \frac{u 2\left(\frac{u_{32}}{a_{2}} K-\frac{n_{3}^{2} u_{21}}{n_{2}^{2} a_{1}} K\right) p_{l}\left(a_{2}\right)-\frac{n_{32}}{a_{2}} q_{l}\left(a_{2}\right)-\frac{u_{21}}{a_{1}} r_{l}\left(a_{2}\right)}{u_{2}\left(\frac{n_{3}^{2}}{n_{2}^{2}} J K+\frac{\sigma_{1} \sigma_{2} u_{21} u_{32}}{n_{2}^{2} a_{1} a_{2}}\right) p_{l}\left(a_{2}\right)+\frac{n_{3}^{2}}{n_{1}^{2}} K q_{l}\left(a_{2}\right)+J r_{l}\left(a_{2}\right)-\frac{n_{2}^{2}}{n_{1}^{2} u_{2}} s_{l}\left(a_{2}\right)}
$$

The following definitions have been used in Eqs. (5) through (7):

$$
\begin{aligned}
& \sigma_{1}=i n_{e f f} l / Z_{0}, \\
& \sigma_{2}=i n_{e f f} l Z_{0}, \\
& u_{21}=\frac{1}{u_{2}^{2}}-\frac{1}{u_{1}^{2}}, \\
& u_{32}=\frac{1}{w_{3}^{2}}-\frac{1}{w_{3}^{2}} ; \\
& u_{j}^{2}=(2 \pi / \lambda)^{2}\left(n_{j}^{2}-n_{e f f}^{2}\right) \quad[j \in(1,2)], \\
& w_{3}=(2 \pi / \lambda)^{2}\left(n_{e f f}^{2}-n_{e f f}^{2}\right), \\
& J=\frac{J_{l}^{\prime}\left(u_{1} a_{1}\right)}{u_{1} J_{l}\left(u_{1} a_{1}\right)}, \\
& K=\frac{K_{l}^{\prime}\left(w_{1} a_{2}\right)}{w_{w} K_{l}\left(w_{3} a_{2}\right)}, \\
& s_{l}(r)=J_{l}^{\prime}\left(u_{2} r\right) N_{l}\left(u_{2} a_{1}\right)-J_{l}^{\prime}\left(u_{2} a_{1}\right) N_{l}^{\prime}\left(u_{2} r\right) \\
& \left.q_{l}(r)=J_{l}\left(u_{2} r\right) N_{l}\left(u_{2} r\right) N_{l}^{\prime} a_{1}\right)-J_{l}\left(u_{2} a_{1}\right)-J_{l}^{\prime}\left(a_{1}\right) N_{l}\left(u_{2} r\right), a_{1} r\left(u_{2} r\right), \\
& r_{l}(r)=J_{l}^{\prime}\left(u_{2} r\right) N_{l}\left(u_{2} a_{1}\right)-J_{l}\left(u_{2} a_{1}\right) N_{l}^{\prime}\left(u_{2} r\right),
\end{aligned}
$$

where $N$ is a Bessel function of the second kind, or the Neumann function. $l$ is azimuthal number. The vector components of the electric field for the cladding modes in the fiber core $(\mathrm{r}<$ a1) are given by 


$$
\begin{aligned}
& E_{r}^{c l}=i A \frac{u_{1}}{2}\left\{J_{2}\left(u_{1} r\right)+J_{0}\left(u_{1} r\right)-\frac{\sigma_{2} \zeta_{0}}{n_{1}{ }^{2}}\left[J_{2}\left(u_{1} r\right)-J_{0}\left(u_{1} r\right)\right]\right\} \exp (i \phi) \exp [i(\beta z-\omega t)] \\
& E_{\phi}^{c l}=A \frac{u_{1}}{2}\left\{J_{2}\left(u_{1} r\right)-J_{0}\left(u_{1} r\right)-\frac{\sigma_{2} \zeta_{0}}{n_{1}{ }^{2}}\left[J_{2}\left(u_{1} r\right)+J_{0}\left(u_{1} r\right)\right]\right\} \exp (i \phi) \exp [i(\beta z-\omega t)] \\
& E_{z}^{c l}=A \frac{u_{1}{ }^{2} \sigma_{2} \zeta_{0}}{n_{1}{ }^{2} \beta} J_{1}\left(u_{1} r\right) \exp (i \phi) \exp [i(\beta z-\omega t)]
\end{aligned}
$$

where $A$ is the field normalization constant.

We focus on the simple case of coupling between two fiber modes $j$ and $k$. At this case, we can assume that the electromagnetic fields of the coupled waveguide can be expressed assume of the eigen modes in each waveguide, such that:

$$
\left.\psi^{(\lambda)}=A(z) \psi_{j}^{(\lambda)} \exp \left(-i \beta_{j \lambda} z\right)+B(z) \psi_{k}^{(\lambda)} \exp \left(-i \beta_{k \lambda} z\right)\right)
$$

where $\psi_{j}^{(\lambda)}$ and $\beta_{j \lambda}$ represents the normalized modal field and propagation constant of the core mode of the fiber, and $\psi_{k}^{(\lambda)}$ and $\beta_{k \lambda}$ represents the normalized modal field and propagation constant of the cladding mode of the fiber for the wavelength $\lambda$. Then according to the coupledmode theory, the reformulated equations for evolution of amplitude of core mode as well as cladding mode are:

$$
\begin{aligned}
& \frac{d A}{d z}=i B(z)\left(K_{k j}{ }^{t}+K_{k j}{ }^{z}\right) \exp \left[-i\left(\beta_{k \lambda}-\beta_{j \lambda}\right) z\right] \\
& \frac{d B}{d z}=i A(z)\left(K_{j k}{ }^{t}+{K_{j k}}^{z}\right) \exp \left[-i\left(\beta_{j \lambda}-\beta_{k \lambda}\right) z\right]
\end{aligned}
$$

where $K_{j k}{ }^{t}$ is the transverse coupling coefficient between modes $\mathrm{j}$ and $\mathrm{k}$ given by

$$
K_{j k}{ }^{t}=\frac{\omega}{4} \int_{0}^{2 \pi} d \phi \int_{0}^{\infty} r d r \Delta \varepsilon(r, z) \cdot E_{j}^{t}(r, \phi) \cdot E_{k}^{t}(r, \phi)
$$

where $\Delta \varepsilon$ is the perturbation to the permittivity. The longitudinal coefficient $K_{j k}{ }^{z}$ is analogous to $K_{j k}{ }^{t}$, but generally $K_{j k}{ }^{2}<<K_{j k}{ }^{t}$ for fiber modes, and thus this coefficient is usually neglected. 
To investigate the coupled effect of the two modes, we need solve equations (2), (5), (12), (13). In most case, it is difficult to solve these equations directly. Numerical methods are used in the project. Newton method can be used to solve equations (2), (5) and a fourth order RungeKutta technique can be used to solve equations (12), (13).

A simulation program was developed based on above model to investigate the pitch length of LPFG. It was found that the period of the LPFG should be about $0.5 \mathrm{~mm}$ or $1.8 \mathrm{~mm}$ to give a resonance wavelength in the $1550 \mathrm{~nm}$ range.

\subsubsection{LPFGs fabrication}

The fabrication of LPFGs relies upon the introduction of a periodic modulation of the optical properties of the fiber. The modulation of the core refractive index has been achieved by ultraviolet (UV) irradiation, ion implantation, irradiation by femtosecond pulses in the infrared, irradiation by $\mathrm{CO}_{2}$ lasers, diffusion of dopants into the core, relaxation of mechanical stress and electrical discharges.

In this project, irradiation by $\mathrm{CO}_{2}$ lasers was used because this would result in thermal LPFGs to survive high temperatures. As shown in Figure 7, LPFG was fabricated by exposing a section of optical fiber to high power $\mathrm{CO}_{2}$ laser irradiation at the wavelength of $10.6 \mu \mathrm{m}$ to introduce periodic refractive index changes inside the optical fiber. The system included a high power $\mathrm{CO}_{2}$ laser (Universal Laser System, OEM-40), computer-controlled precision threedimensional translation stages (Newport, LTA-HS), fiber support mechanisms, an on-line signal monitoring system, and the computer program to coordinate the entire fabrication process. The picture of the $\mathrm{CO}_{2}$ laser based LPFG fabrication system is shown in Figure 8. 


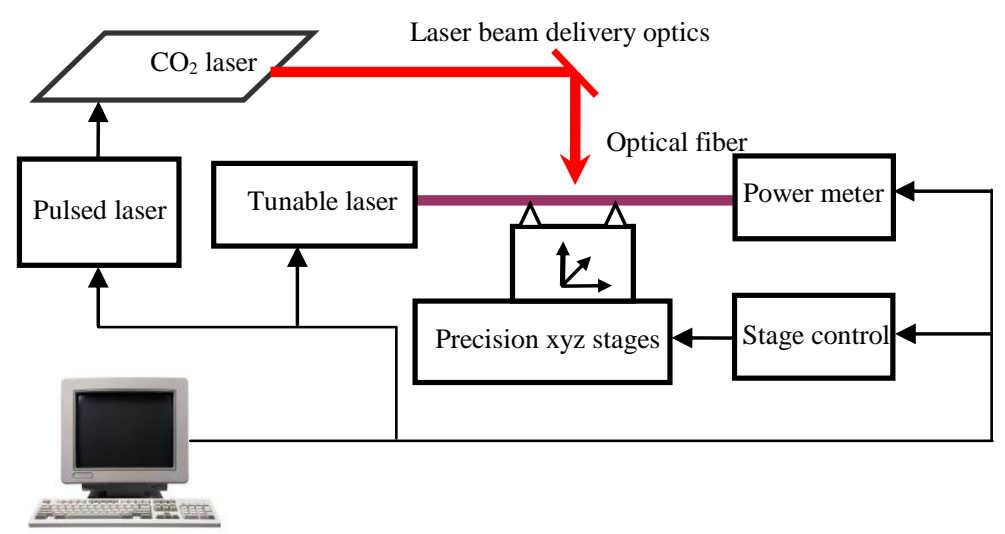

Figure 7 Schematic illustration of the $\mathrm{CO} 2$ laser based LPFG fabrication system.

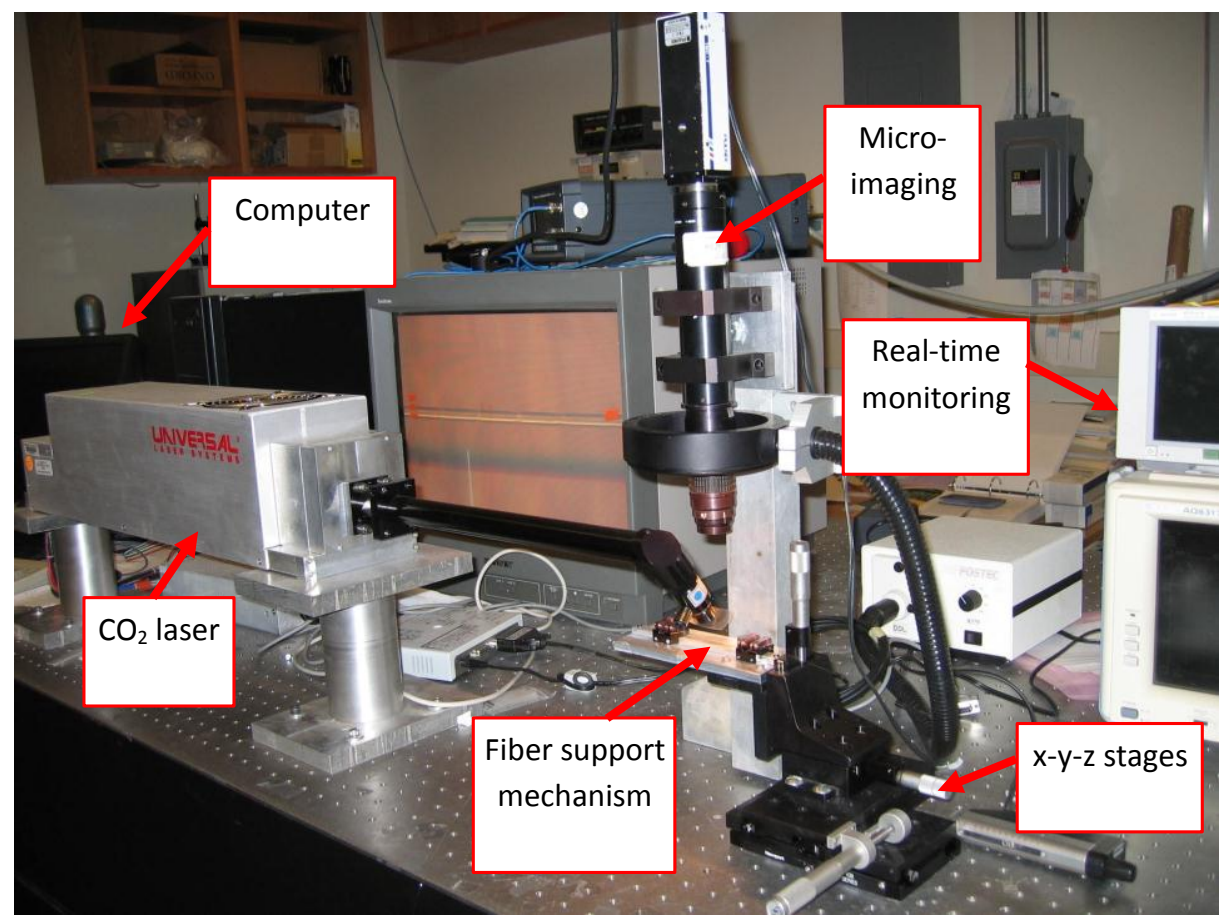

Figure 8 Photograph of the $\mathrm{CO}_{2}$ laser based LPFG fabrication system.

The $\mathrm{CO}_{2}$ laser power was controlled by a computer program so that it could be precisely controlled to step through a pre-designed power-time trajectory. It was found that the optimal power of $\mathrm{CO}_{2}$ laser irradiation was about $7 \mathrm{~W}$. Too strong power resulted in unacceptable damage of the fiber while too weak power did not provide enough refractive index modification.

The $x-y-z$ stage was controlled by the computer to allow accurate positioning of the optical 
fiber toward the laser beam. The current accuracy of the motorized stage was less than $1 \mu \mathrm{m}$, which was found high enough to fabricate high quality LPFGs.

The on-line monitoring system included a tunable laser (HP81640A) and a power meter (HP8163A). By stepping the laser wavelength from $1510 \mathrm{~nm}$ to $1640 \mathrm{~nm}$, the resonance spectrum of the fabricated LPFG was monitored as the motorized stage move the fiber to a pre-designed location for laser radiation.

Figure 9(a) shows the microimage of a typical thermal LPFG, where the laser irradiation marks can be clearly identified on the fiber. The corresponding transmission spectrum of this thermal LPFG is shown in Figure 9(b). High quality thermal LPFGs can now be fabricated using the system with very good repeatability. With a period of about $0.5 \mathrm{~mm}$ and about 50 points of irradiation, the fabricated thermal LPFGs had a typical low loss (typically $2 \mathrm{~dB}$ ), a resonance wavelength around $1550 \mathrm{~nm}$, and a resonance peak of above $12 \mathrm{~dB}$. If the number of exposure points increased to about 100 , the resonance peak could reach $20 \mathrm{~dB}$. The resonance wavelength could also be tuned in the range from $1510 \mathrm{~nm}$ to $1640 \mathrm{~nm}$ by slightly changing the period. 

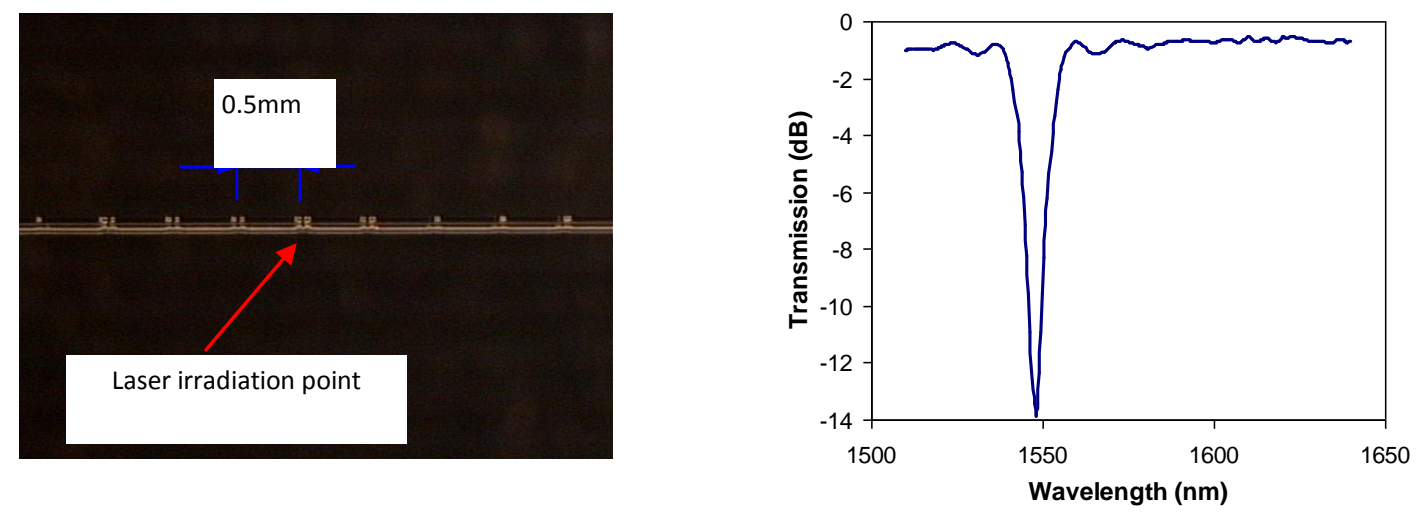

Figure 9 Thermal LPFG, (a) microimage of a fabricated thermal LPFG, (b) typical transmission spectrum of a thermal LPFG.

\subsubsection{LPFGs optimization}

The following works has been conducted to improve the quality of the grating as well as the controllability of the fabrication processes. These include: 1) investigate the best power-time trajectory, 2) investigate the optimal number of irradiation points to produce the maximum resonance strength, and 3) investigate the period-resonance wavelength relations.

With better control of $\mathrm{CO}_{2}$ laser irradiation and optimized grating periods, high quality LPFGs now can be fabricated routinely in our lab in less than 20 minutes. As shown in Figure 10, the fabricated LPFG has typical resonance strength higher than $25 \mathrm{~dB}$, a full-width-at-halfmaximum (FWHM) less than $2 \mathrm{~nm}$, and side ripples less than $2 \mathrm{~dB}$. The fabrication of these high quality LPFGs is essential to the success of the proposed program because it provides a robust platform for the sensor development. 


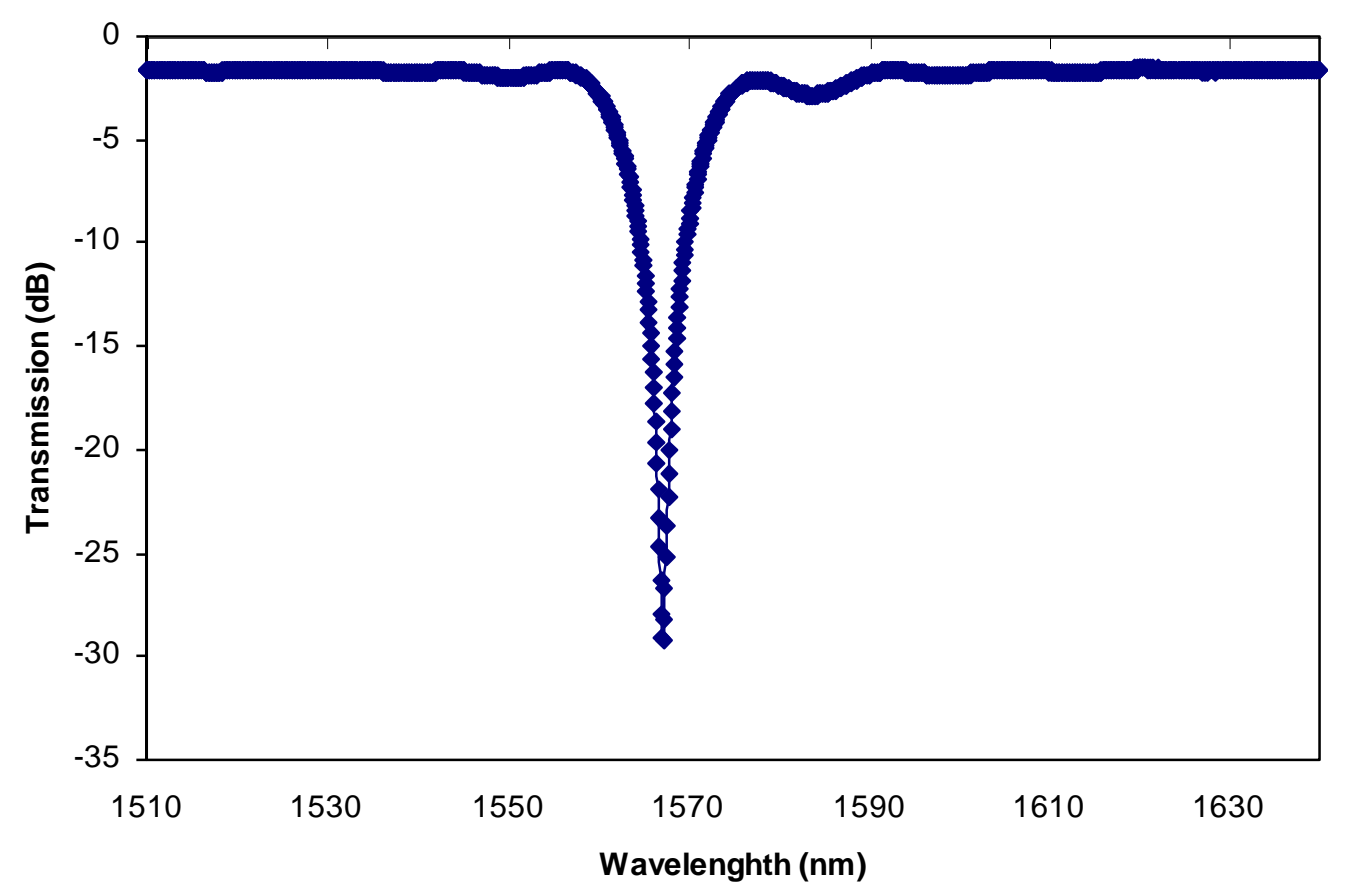

Figure 10 Transmission spectrum of the typical LPFG fabricated in our lab.

Figure 11 shows the relation between the grating period and the resonance wavelength. By varying the grating period in the range of $505-550 \mathrm{~nm}$, the resonance wavelength of the LPFG can be tuned to any chosen specific wavelength within the interrogation spectrum from 1510 to $1640 \mathrm{~nm}$. This spectrum range is mainly limited by the currently tunable laser available in our lab. Another tunable laser is currently under purchasing which will further expand the interrogation spectrum range. The transmission spectra of these LPFGs are shown in Figure 11. 


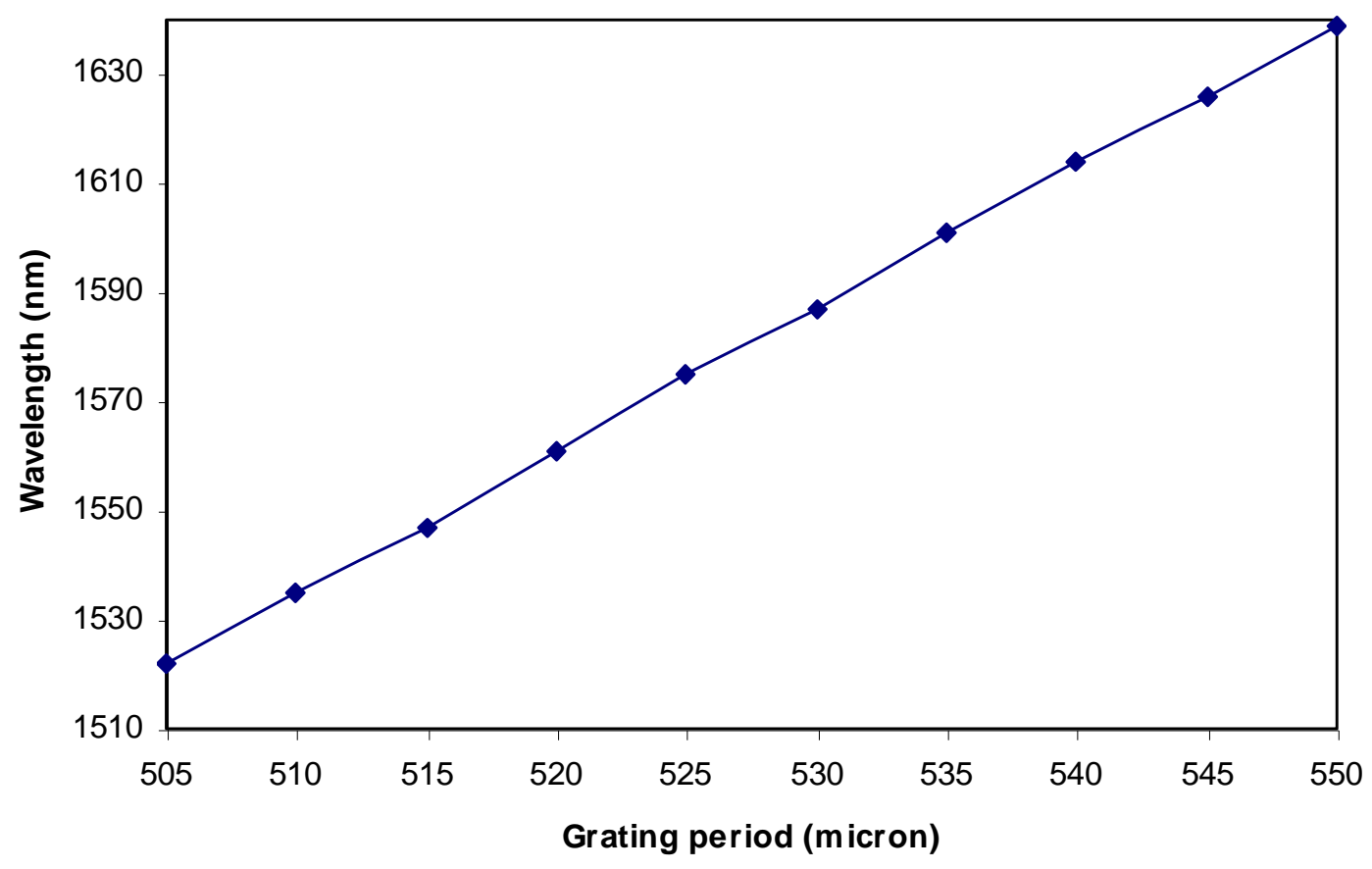

Figure 11 LPFG resonance wavelength as a function of the grating period.

Hundreds of these gratings with different periods (thus different resonance wavelengths) have been fabricated. Forty selected gratings have been sent to the ASU and UC teams for nanomaterial coating. The coated LPFGs will be either evaluated locally by these two teams, or will be sent back to Missouri S\&T for testing.

Besides using the Corning SMF-28 singleode fiber, fibers made by other venders (e.g., the Lucent Truewave singlemode fiber) is currently under investigation for LPFG fabrication. The reason for trying different fibers is to investigate potential better thermal stability of the LPFG. 


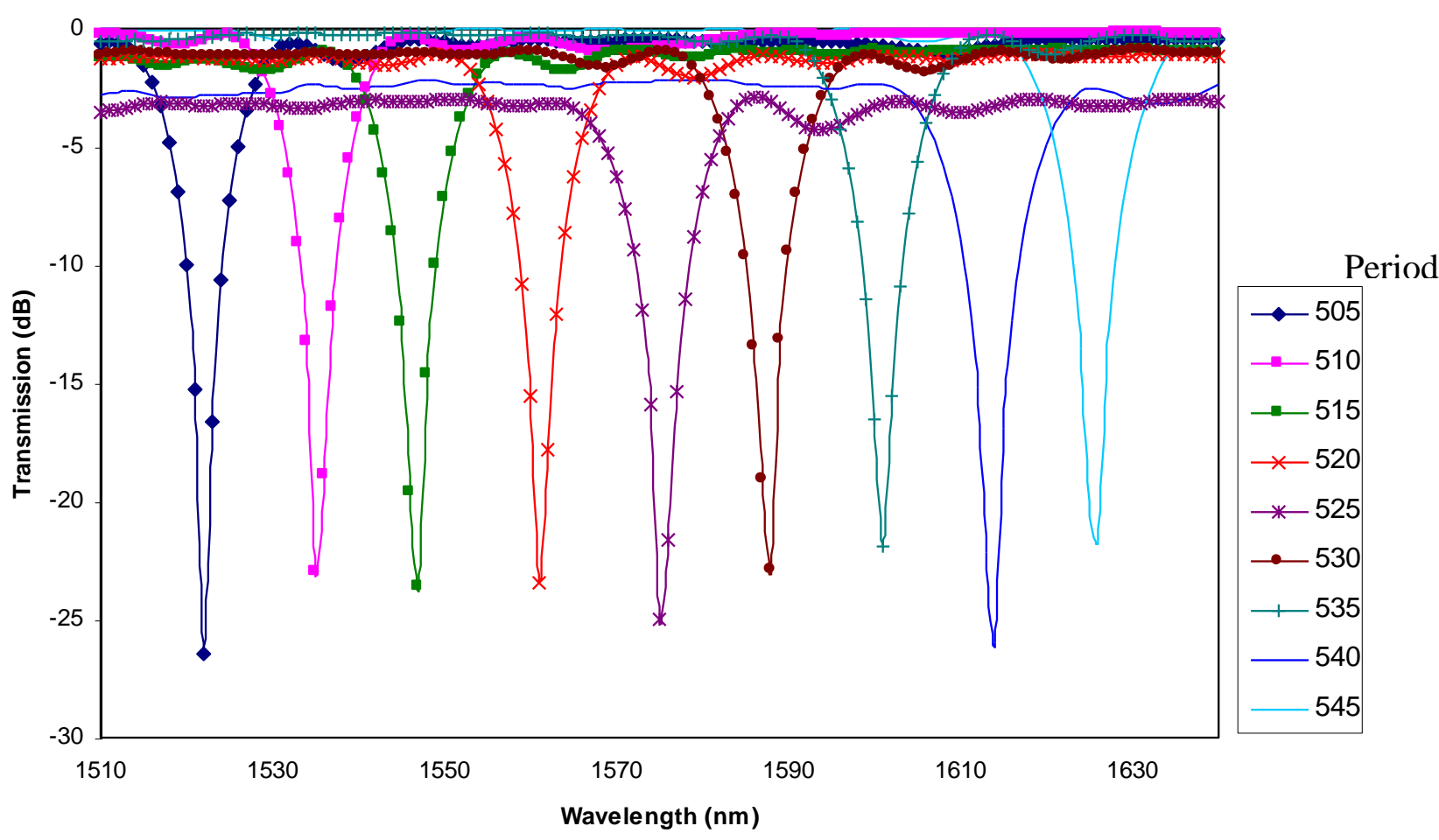

Figure 12 Transmission spectra of the LPFGs with different period corresponding resonance wavelengths shown in Figure 14.

\section{$\underline{\text { 1.2.4 Measurement of } \mathrm{CO}_{2}}$ Laser Irradiation Induced Refractive Index Modulation in}

\section{Singlemode Fibers for LPFG Design and Fabrication}

The amount of refractive index modulation inside a fiber core is one of the most critical variables that determine the transmission characteristic of an LPFG. Accurate knowledge of the $\mathrm{CO}_{2}$ laser irradiation caused refractive index changes can thus help us adjust the $\mathrm{CO}_{2}$ laser parameters such as the power and pulse duration during LPFG fabrication to achieve desired performance. Modeling of the fabricated LPFG predicted that the magnitude of refractive index change caused by $\mathrm{CO}_{2}$ laser irradiation was at a scale of $10^{-4}$. However, due to the very small change, accurate measurement of the refractive index modulation has been a great challenge. As 
a result, the process of using a $\mathrm{CO}_{2}$ laser to fabricate LPFGs still remains in the trail-and-error stage.

The $\mathrm{CO}_{2}$ laser irradiation induced refractive index change on an optical fiber is known as a result of residual stress relaxation caused by the localized thermo shocks. During the drawing process, residual stress is developed inside the fiber as a result of radial variations of the thermal expansion and viscoelastic properties. The refractive index of an optical fiber thus depends on the amount and distribution of the residual stress. Kim et al. used a Mach-Zehnder interferometer constructed by an LPFG pair to measure the $\mathrm{CO}_{2}$ laser induced refractive change. In the reported approach, the two LPFGs were separated by a section of fiber with a length of about $15 \mathrm{~cm}$. The first LPFG coupled part of the light energy into the cladding mode while the rest remained inside the fiber core. After passing the middle section, the cladding and core modes were recoupled and mixed at the second LPFG, resulting in interference fringes inside the transmission spectrum of the grating. The phase of the interference signal was proportional to the optical path difference (OPD) between the cladding and core modes. By monitoring the spectral shift of the interference fringe, it was found that the laser irradiation induced refractive index change was negative and in the range from $-8.0 \times 10^{-5}$ to $-2.1 \times 10^{-4}$, depending on the fiber type, laser power, as well as the applied tension.

In this paper, we report a new method based on a simple fiber Fabry-Perot interferometer to measure the $\mathrm{CO}_{2}$ laser irradiation-induced refractive index changes inside the fiber core with various laser pulse durations for the purpose of design and fabrication of LPFG. The measured refractive index modulations were used to simulate the LPFG transmission spectra. Tension free point-to-point laser exposure was used to fabricate LPFGs using the same conditions as in refractive index measurements. The simulated spectra were found in good agreement with those 
of real devices.

\section{Principle and theory}

\section{Refractive index modulation and LPFG modeling}

A LPFG can be formed by exposing a single mode optical fiber to periodic $\mathrm{CO}_{2}$ laser irradiations, which change the fiber refractive index in the exposed areas as a result of the localized laser heating. As shown in Figure 13, the shaded areas represent the portions exposed to $\mathrm{CO}_{2}$ laser beam with a Gaussian-shaped intensity distribution. Assuming that the laser irradiation produces a Gaussian-shaped index perturbation profile along the axial direction (z) of the optical fiber, the refractive modulation function is given by

$$
\Delta n=\Delta n_{\max } \exp \left(-\frac{z^{2}}{2 \sigma^{2}}\right)
$$

where $2 \sigma$ is the full width of the Gaussian-shaped index perturbation profile, and $\Delta \mathrm{n}_{\max }$ is the peak refractive index change in the core at the position corresponding to the center of the laser beam.

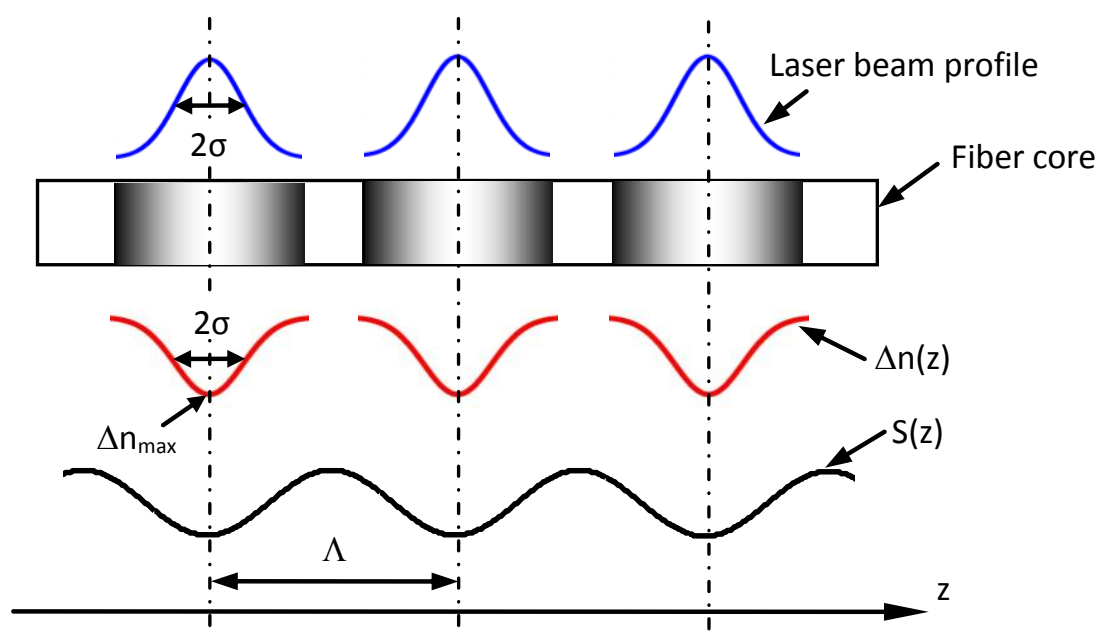

Figure 13 Periodic $\mathrm{CO} 2$ laser irradiation induced refractive index modulation in an optical fiber. 
Assuming that the period of laser exposures is $\Lambda$, the axial refractive index modulation function $\mathrm{S}(\mathrm{z})$ can be approximated by the first two terms of Fourier series of the periodic Gaussian-shaped perturbation in LPFG modeling. That is

$$
\Delta n(z) \approx S(z)=s_{0}+s_{1} \cos \left(\frac{2 \pi}{\Lambda} z\right)
$$

where $s_{0}$ and $s_{1}$ are the coefficients of the first two terms of the Fourier series, respectively, given by

$$
\begin{aligned}
& s_{0}=\Delta n_{\max } \frac{1}{\Lambda} \int_{-\Lambda / 2}^{\Lambda / 2} \exp \left(-\frac{z^{2}}{2 \sigma^{2}}\right) d z, \\
& s_{1}=\Delta n_{\max } \frac{2}{\Lambda} \int_{-\Lambda / 2}^{\Lambda / 2} \exp \left(-\frac{z^{2}}{2 \sigma^{2}}\right) \cos \left(\frac{2 \pi}{\Lambda} z\right) d z .
\end{aligned}
$$

The transmission characteristics of an LPFG can be determined once we know the fiber parameters, modulation function $\mathrm{S}(\mathrm{z})$, the period and the grating length (or the number of irradiation points). In Eqs. (3) and (4), $\sigma$ can be measured using a laser beam profiler, and the period $\Lambda$ is a variable to be controlled during grating fabrication. The only unknown to be measured is the peak refractive index change $\Delta \mathrm{n}_{\max }$.

\section{Measurement of refractive index modulation using an interferometer}

The peak refractive index change can be measured using a fiber Fabry-Perot interferometer as schematically shown in Figure 14. The light from a tunable laser is directed into a fiber segment through a $3 \mathrm{~dB}$ fiber coupler. With both ends cleaved, the fiber piece operates as a 
Fabry-Perot interferometer in which the reflections at the two endfaces, coupling back through the lead-in fiber and counter propagating through the $3 \mathrm{~dB}$ coupler, superimposed to form an interference signal at the optical power meter. By stepping the tunable laser through its available wavelength range and coordinating the signal detection at the power meter using a computer, the interference spectrum of the Fabry-Perot interferometer can be recorded. When exposed to $\mathrm{CO}_{2}$ laser irradiations, the fiber segment changes its refractive index, resulting in a phase shift in the interference signal. The $\mathrm{CO}_{2}$ laser induced fiber refractive index change can thus be calculated based on the amount of phase shift after laser irradiation.

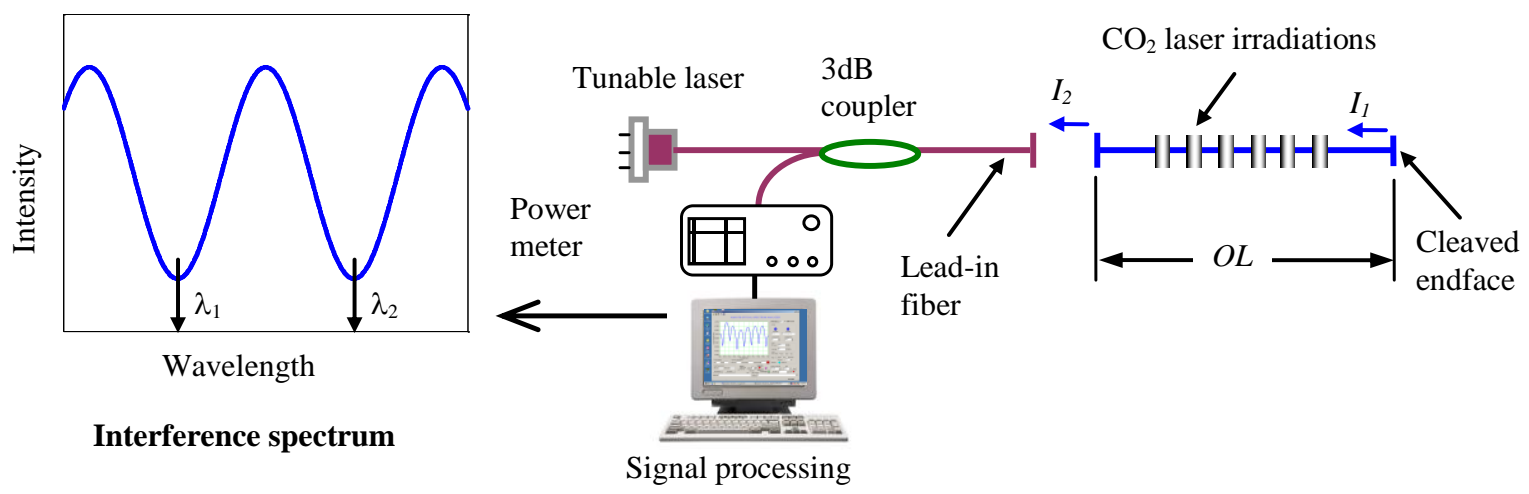

Figure 14 Fiber Fabry-Perot interferometer for measurement of refractive index modulation

Assuming that the two reflections from the cleaved fiber segment endfaces have the intensities of $\mathrm{I}_{1}$ and $\mathrm{I}_{2}$, respectively, the interference signal I generated by these two reflections is given by:

$$
I=I_{1}+I_{2}+2 \sqrt{I_{1} I_{2}} \cos \left(\frac{4 \pi}{\lambda}(O L)+\phi_{0}\right),
$$

where OL is the optical length of the fiber segment, defined as the product of the length and the refractive index of the fiber core; $\phi_{0}$ is the initial phase of the interference; $\lambda$ is the optical 
wavelength in vacuum.

As shown in Figure 14, the two adjacent valleys at $\lambda_{1}$ and $\lambda_{2}$ in the interference spectrum have a phase difference of $2 \pi$, that is:

$$
\left(\frac{4 \pi}{\lambda_{1}} O L\right)-\left(\frac{4 \pi}{\lambda_{2}} O L\right)=2 \pi
$$

Therefore, the initial optical length of the fiber segment can be calculated using the following equation:

$$
O L=\frac{\lambda_{1} \lambda_{2}}{2\left(\lambda_{1}-\lambda_{2}\right)}
$$

For LPFGs fabricated without applying tension to the optical fiber, the physical length of the fiber does not vary after laser irradiations. The change in optical length is mainly caused by the laser irradiation induced refractive index change inside the fiber core. Assuming a Gaussianshaped laser beam profile as denoted by Eq. (4), the single laser irradiation induced change in optical refractive index $\left(\Delta \mathrm{OL}_{\text {single }}\right)$ can be expressed as:

$$
\Delta O L_{\text {single }}=\int_{-\infty}^{+\infty} \Delta n_{\max } \exp \left(-\frac{z^{2}}{2 \sigma^{2}}\right) d z=\Delta n_{\max } \sigma \sqrt{2 \pi}
$$

For multiple-point laser irradiations (with the same laser conditions) at different locations, the accumulated change in optical length $(\Delta \mathrm{OL})$ along the fiber section is given by

$$
\Delta O L=m \cdot\left(\Delta O L_{\text {single }}\right)=\left(\Delta n_{\max } \sigma \sqrt{2 \pi}\right) m
$$

where $\mathrm{m}$ is the total number of laser irradiations.

When the amount of optical length change is small so that the phase shift is less than $2 \pi$, the phase ambiguity issue can be avoided. The relative optical length change can be calculated based 
on the spectral shift of the interferogram at the featured points such as the peak, valley and the center of the interference fringes, given by

$$
\Delta O L=\frac{\Delta \lambda_{1}}{\lambda_{1}} O L,
$$

where $\lambda_{1}$ and $\Delta \lambda_{1}$ are the wavelength and the shift of wavelength at the corresponding feature point, respectively.

Combining Eqs. (9) and (10), one finds the peak refractive index change, given by

$$
\Delta n_{\max }=\frac{1}{m \sigma \sqrt{2 \pi}} \frac{\Delta \lambda_{1}}{\lambda_{1}} O L
$$

An alternative way of calculating the peak refractive index change is directly based on Eq. (9) in which the change in optical length is a linear function of the number of laser exposures and the slope of the line is linearly proportional to $\Delta \mathrm{n}_{\max }$. Experimentally, one can measure the optical length change after a various number of laser exposures and curve-fit the measurement results into a line. The slope of the fitted line can thus be used to calculate $\Delta \mathrm{n}_{\max }$. This method uses multiple data points in calculation and effectively reduces the measurement uncertainty.

Once $\Delta \mathrm{n}_{\max }$ is determined, the refractive index modulation coefficients $\left(\mathrm{s}_{0}\right.$ and $\left.\mathrm{s}_{1}\right)$ can be calculated according to Eqs. (3) and (4), and the LPFG transmission spectrum can be determined by computer simulation.

\section{Experiments}

\section{LPFG fabrication}

The method used to write LPFGs in this study was similar to that has been reported by others. As shown in Figure 15, a focused $\mathrm{CO}_{2}$ laser beam (Synrad Inc., Firestar V20, $\lambda=10.6$ 
$\mu \mathrm{m}$ ) was transversely loaded onto the single mode optical fiber (Corning SMF-28e) mounted on a three-dimensional (3D) translation stage. The laser beam was focused by a cylindrical $\mathrm{ZnSe}$ lens with a focal length of $50 \mathrm{~mm}$. The cylindrical axis of the lens was aligned to produce a focused laser line crossing the fiber perpendicularly. The width $(2 \sigma)$ of the focused laser beam at the fiber position was measured to be about $180 \mu \mathrm{m}$. Controlled by a computer, the translation stage moved the fiber at fixed step for laser exposure, resulting in a periodic refractive index modulation in the fiber core. The laser power and the exposure time were also controlled by the computer. During grating fabrication, a tunable laser (HP 81642A) with a spectral range from 1510 to $1640 \mathrm{~nm}$ and an optical power meter (HP 81618A) were used to monitor the transmission spectrum of the grating.

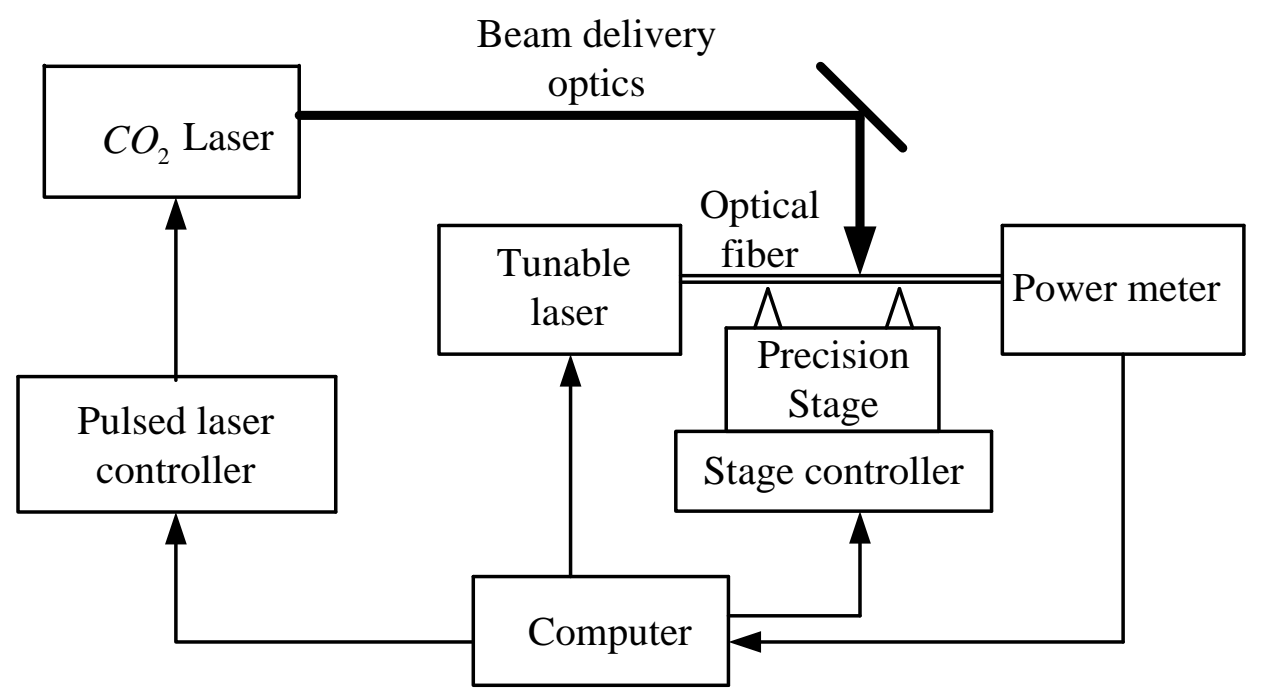

Figure 15 Block diagram of CO2 laser based LPFG fabrication system

\section{Measurement of $\mathrm{CO}_{2}$ laser induced refractive index changes}

A fiber Fabry-Perot interferometer was constructed according to Figure 14. The fiber used in the experiment was Corning SMF-28e single mode fiber. A small segment of fiber with a length of about $20 \mathrm{~mm}$ was prepared by cleaving both sides and cleaned carefully in an ultrasonic 
acetone bath. The fiber sample was mounted on the 3D translation stage for laser exposures. The lead-in fiber was brought in to a very close distance $(\sim 2 \mu \mathrm{m})$ to the fiber segment. After careful alignment, the interference spectrum of the fiber segment was taken by scanning the tunable laser with a resolution of $1 \mathrm{pm}$ per step.

The fiber segment was exposed to $\mathrm{CO}_{2}$ laser irradiations at different locations by moving the stage along the fiber axial direction. The distance from the laser to the fiber segment was kept to be the same as in the grating fabrication. The axial separation between two adjacent exposure locations was about $525 \mu \mathrm{m}$ but not exactly the same. In fact, the axial distance was varied randomly to avoid grating effects in the interference spectrum. The laser power was set to a constant value of $8 \mathrm{~W}$ for all experiments but the laser exposure time was varied to investigate the possibility of controlling the laser-induced refractive index changes.

For each fiber sample used in experiments, an initial spectrum was taken before laser irradiation and a spectrum was recorded after every eight (8) laser exposures. The reason of recording the spectrum after every eight laser exposures was to allow enough phase shift accumulation within the $2 \pi$ unambiguity limit. The length of the fiber segment $(\sim 20 \mathrm{~mm})$ allowed a total of 24 laser exposures separated at a distance of about $525 \mu \mathrm{m}$. Therefore, 4 interference spectra were recorded on each fiber sample. The initial optical length (OL) of the fiber segment was calculated using Eq. (7). The changes in optical length were calculated using Eq. (10) and the peak refractive index change $\left(\Delta \mathrm{n}_{\max }\right)$ was determined based on the slope of the line-fitted measurement data.

\section{LPFG modeling}

Modeling of the LPFG mainly followed the methods and procedures laid out in previous 
work, where the coupled-mode theory was applied to treat the interaction between the fundamental core mode to various cladding LP modes, and the transfer matrix method was used in the calculations. It has been pointed out that gratings produced by $\mathrm{CO}_{2}$ laser irradiation may have non-uniform azimuthal refractive index profile in the cross section of a fiber. However, the modeling work in this paper only considered an LPFG with uniform azimuthal refractive index profile for simplicity.

Here we only highlight the major steps. First, the propagation constants $(\beta)$ and the amplitude coefficients of the fundamental core mode $\left(\mathrm{LP}_{01}\right)$ and the cladding modes up to the eighth order $\left(\mathrm{LP}_{02}, \mathrm{LP}_{03}, \ldots, \mathrm{LP}_{08}\right)$ were calculated in the wavelength range from 1510 to $1640 \mathrm{~nm}$. Second, the modulation coefficients $s_{0}$ and $s_{1}$ in Eqs. (3) and (4) were calculated based on the measured $\Delta \mathrm{n}_{\max }$. The coupling coefficients of these modes were computed correspondingly. Third, the optical powers of the modes were computed in the wavelength range using the transfer matrix formed based on the coupled model equations. Finally, the transmission spectrum of the grating was calculated and plotted.

\section{Results and discussions}

Figure 16(a) shows the initial interference spectrum and those after 8, 16, and 24 points laser irradiations with the laser power of $8 \mathrm{~W}$ and exposure time of $125 \mathrm{~ms}$. The large fringe visibility indicated high-quality of the interference signals. Since each laser exposure induced a small amount of power loss, the fringe visibility of the interference signal reduced as the number of laser exposures increased. The initial optical length of this particular fiber segment was found to be $31.806 \mathrm{~mm}$, corresponding to a fiber length of $21.663 \mathrm{~mm}$.

The $\mathrm{CO}_{2}$ laser irradiation induces a negative refractive index change in an optical fiber as a 
result of residual stress relaxation. The interference spectrum shifts to the short wavelength as the number of laser exposures increases. By tracing the interferogram shifts, we calculated the changes in optical length of the fiber segment based on Eq. (9). The results, as a function of the number of laser exposures, are plotted in Figure 16(b), where the measured data points fit nicely into a line with a slope of $-160.035 \mathrm{~nm}$ per laser exposure. The laser induced $\Delta \mathrm{n}_{\max }$ was calculated to be $-7.12 \times 10^{-4}$. Based on Eq. (3) and (4), the modulation coefficients $s_{0}$ and $s_{1}$, were determined to be $-3.04 \times 10^{-4}$ and $-1.82 \times 10^{-4}$, respectively.
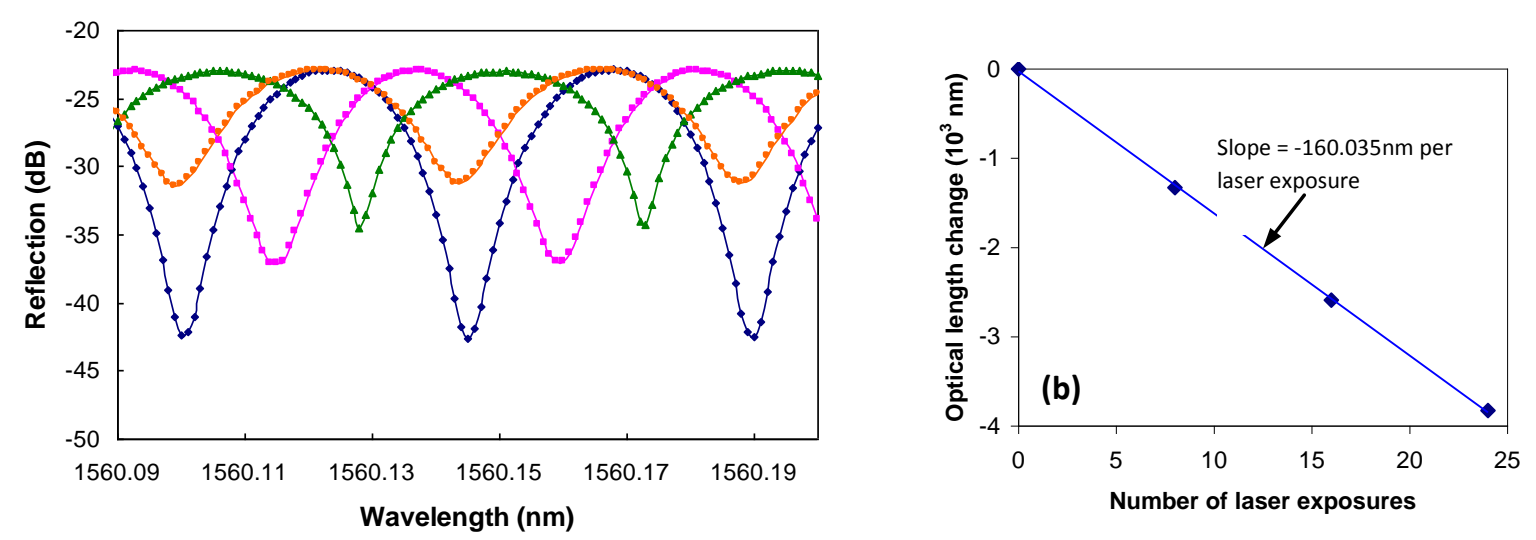

Figure 16 Optical length changes of fiber segment caused by $\mathrm{CO} 2$ laser irradiations with the power of $8 \mathrm{~W}$ and exposure time of $125 \mathrm{~ms}$; (a) interference spectra; (b) change in OL as a function of the number of laser exposures.

To investigate the possibility of controlling the laser-induced refractive index changes. We repeated above experiments by varying the exposure time of laser irradiation while keeping all other parameters the same. Figure 17(a) shows the change in fiber optical length as a function of the number of laser exposures with four different exposure times of 50,75, 100 and $125 \mathrm{~ms}$, respectively. As the laser exposure time increased, the change in fiber optical length became more negative (i.e., increasing magnitude). The corresponding peak refractive index changes $\left(\Delta \mathrm{n}_{\max }\right)$ are plotted in Figure 17(b). We found that the laser-induced refractive index change is a 
very sensitive function of the laser exposure time. The magnitude of $\Delta \mathrm{n}_{\max }$ increased with the increase of laser exposure time, following almost a linear relation. Because the laser exposure time can be accurately varied by computer control (e.g., at the resolution of $1 \mathrm{~ms}$ in our current laser system), it is possible to obtain a preferred refractive index modulation for fabrication of LPFGs with desired performance.
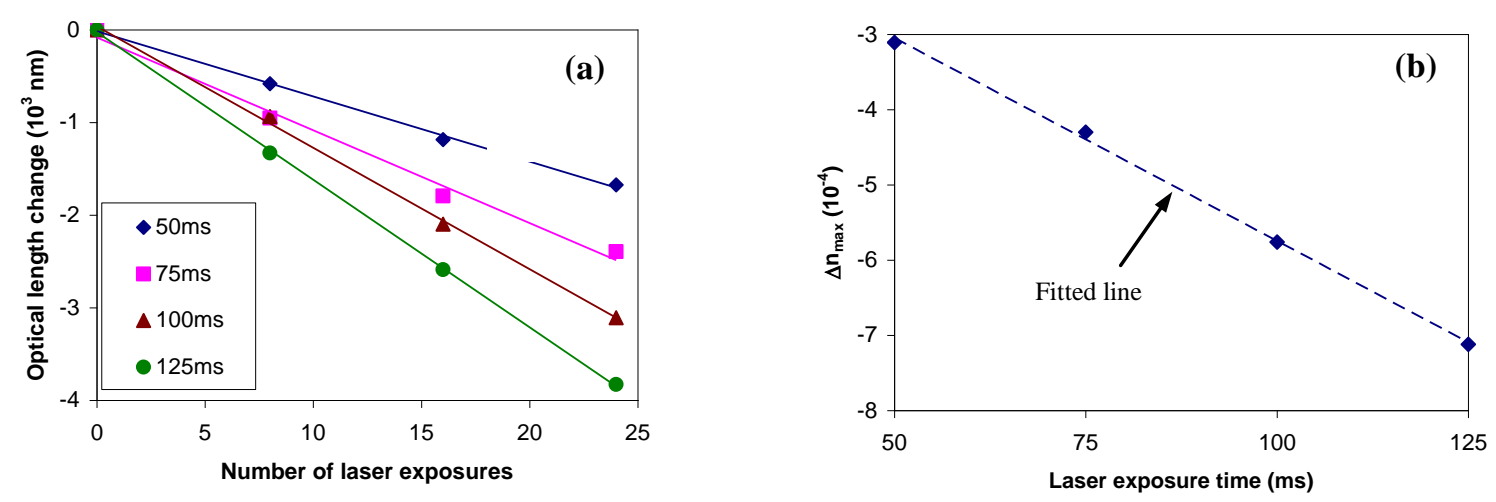

Figure $17 \mathrm{CO} 2$ laser irradiation induced fiber refractive index changes at various laser exposure times (50, 75, 100 and $125 \mathrm{~ms}$, respectively); (a) OL change as a function of the number of laser exposures; (b) $\Delta$ nmax as a function of laser exposure time.

The measured refractive index modulation coefficients were used to calculate the grating spectra in the wavelength range of 1510-1640 $\mathrm{nm}$. The following Corning SMF-28e fiber parameters from datasheet were used in the calculations: group effective refractive index of core $\mathrm{n}_{\mathrm{eff}}=1.4682$ at $1550 \mathrm{~nm}$, core and cladding index contrast $0.36 \%$, and the core and cladding diameters of 8.2 and $125 \mu \mathrm{m}$, respectively. The period and length of the LPFG were $525 \mu \mathrm{m}$ and $46.725 \mathrm{~mm}$ (total of 89 points), respectively. It was found that the resonance peak was a result of light coupling from the fundamental $\mathrm{LP}_{01}$ core mode to the $\mathrm{LP}_{05}$ cladding mode in the observing wavelength range. We also fabricated four LPFGs using the parameters corresponding to each simulation case for the purpose of verification. The simulated and measured transmission spectra are plotted in Figure $\mathbf{1 8}$ where the background losses of the fabricated gratings were around 1 
$\mathrm{dB}$ and subtracted from their original spectra for easy examination. There were large differences among the simulated and measured transmission spectra As detailed in the insert tables, the measured resonant wavelengths were $1608,1595,1579,1561 \mathrm{~nm}$ corresponding to the four different laser exposure times of 50, 75, 100 and $125 \mathrm{~ms}$, respectively The simulated resonant wavelengths, however, were at 1630, 1617, 1600, and $1580 \mathrm{~nm}$. In addition, large differences existed in the resonance peak strengths.

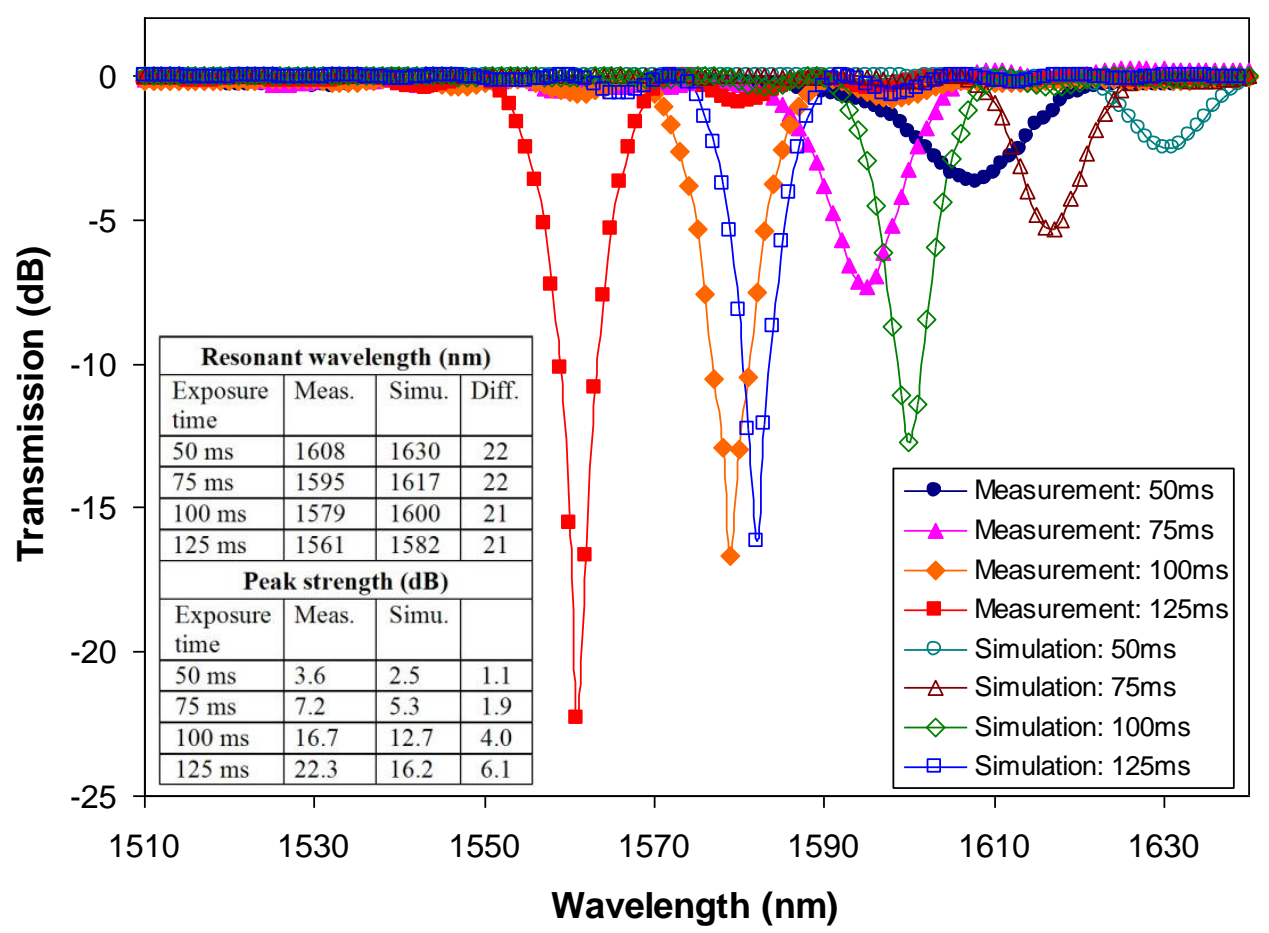

Figure 18 Comparison between the measured and simulated transmission LPFG spectra. Fiber parameters (Corning SMF-28e) from datasheet were used in the simulations. Inset table: detailed comparisons between measured and simulated resonant wavelengths and peak strengths.

However, it is interesting to note that the difference in resonant wavelength between the measured and simulated spectra of each corresponding case was almost constant, 21 or $22 \mathrm{~nm}$. The resonant wavelength is governed by the Bragg condition, given by: 


$$
\lambda_{\text {res_B }}=\Lambda\left(n_{\text {eff }, 01}-n_{\text {eff }, 05}\right)
$$

where $\lambda_{\text {res_B }}$ is the Bragg resonant wavelength, $n_{\text {eff, } 01}$ and $n_{\text {eff }, 05}$ are the effective refractive index of the $\mathrm{LP}_{01}$ core mode and $\mathrm{LP}_{05}$ cladding mode, respectively. A constant resonant wavelength offset between the simulation and the measured spectra in resonant wavelength suggests a offset in $\mathrm{n}_{\mathrm{eff}, 01}$ and $\mathrm{n}_{\mathrm{eff}, 05}$, which both depend on the fiber parameters. We modified the core-cladding index contrast slightly from the $0.36 \%$ to $0.355 \%$. We repeated the simulations using the new core-cladding index contrast value. The use of modified fiber parameter resulted in a much better matched between the simulated and measured spectra in all four cases. As detailed in the embedded table, the difference in resonance wavelength was within $2 \mathrm{~nm}$ and deviation of resonance peak strength was within $1.1 \mathrm{~dB}$.

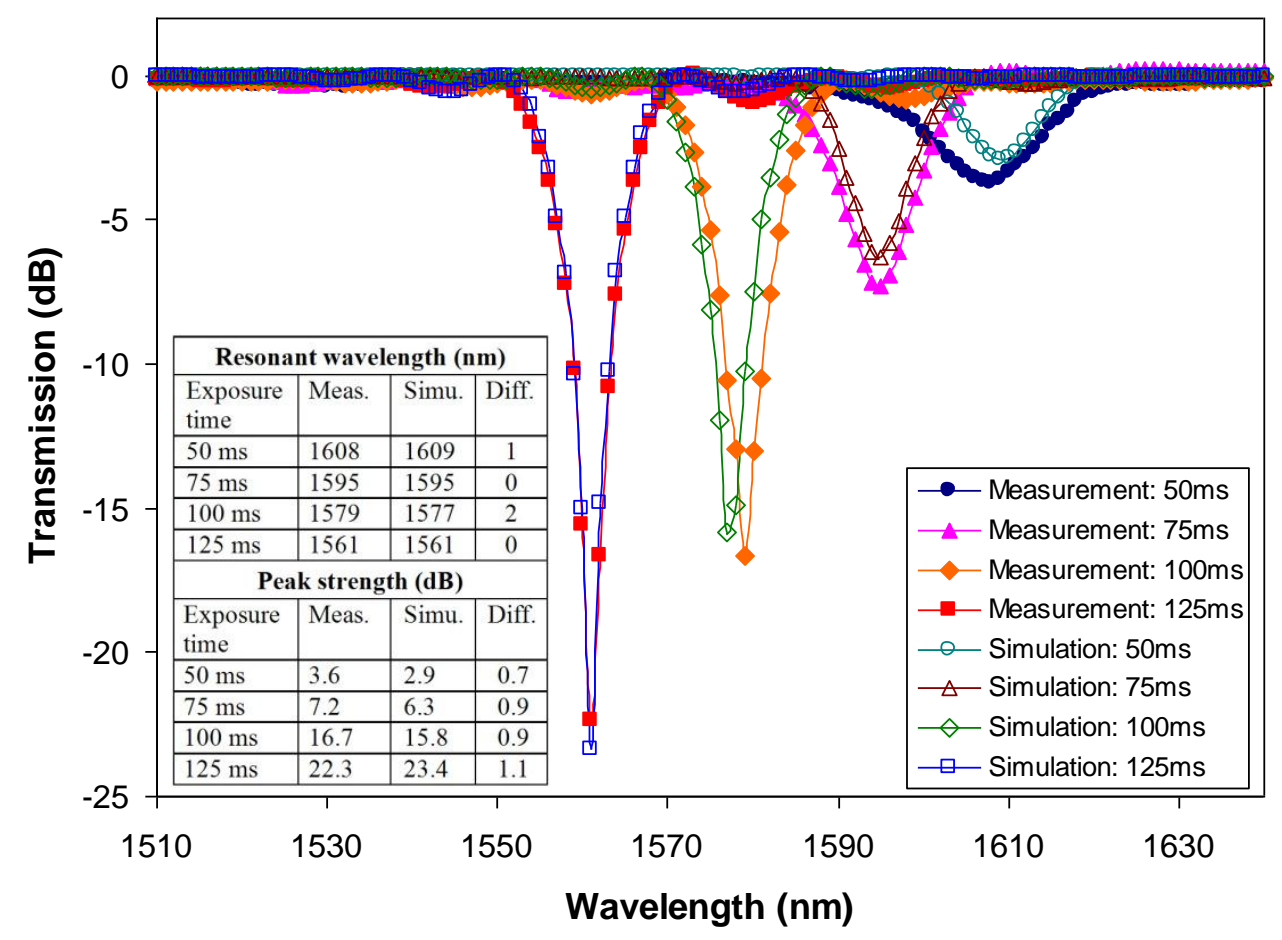

Figure 19 Comparison between the simulated (with slightly modified core-cladding index contrast) and measured transmission LPFG spectra. Inset table: detailed comparisons between measured and simulated resonant wavelengths and peak strengths. 
As indicated in Figure 19, the resonant wavelength shifted to a large wavelength and the peak strength reduced. This can be explained by the modify Bragg condition:

$$
\left(\frac{2 \pi}{\lambda_{\text {res }}} n_{\text {eff }, 01}+s_{0} \zeta_{01,01}(\lambda)\right)-\left(\frac{2 \pi}{\lambda_{\text {res }}} n_{\text {eff }, 05}+s_{0} \zeta_{05,05}(\lambda)\right)=\frac{2 \pi}{\Lambda}
$$

which can be rewritten as:

$$
\lambda_{\text {res }}=\frac{2 \pi\left(n_{e f f, 01}-n_{e f f, 05}\right)}{\frac{2 \pi}{\Lambda}-s_{0}\left(\zeta_{01,01}-\zeta_{05,05}\right)}
$$

where $\lambda_{\text {res }}$ is the resonant wavelength, $\zeta_{01,01}$ and $\zeta_{05,05}$ are the self-coupling coefficients of the $\mathrm{LP}_{01}$ and $\mathrm{LP}_{05}$ modes, respectively.

In Eq. (14), $\mathrm{n}_{\mathrm{eff}, 01}, \mathrm{n}_{\mathrm{eff}, 05}, \zeta_{01,01}$ and $\zeta_{05,05}$ are constants for the chosen fiber at a specific wavelength and the period $\Lambda$ is a fixed value. $\zeta_{01,01}$ is always larger than $\zeta_{05,05}$ due to its large amplitude inside the fiber core, and $\mathrm{s}_{0}$ is a negative value because of the negative value of laser induced refractive index change. As indicated in Figure 17(b), the magnitude of $\mathrm{s}_{0}$ deceases with the reduced laser exposure time. As a result, the LPFG resonant wavelength $\lambda_{\text {res }}$ moves to the long wavelength region (i.e., red shift) when the laser exposure time decreases.

There were some noticeable residual disparities in the simulated and measured LPFG spectra shown in Figure 19, especially in the width of resonance peaks, and the difference became more pronounced at shorter exposure times (or smaller refractive index modulations). In addition to the measurement errors, we believe that the mismatches can be attributed to the following two major reasons. First, in the current simulation model, we assume that the refractive index modulation has a uniform azimuthal distribution in the fiber cross section. However, it has been suggested that the $\mathrm{CO}_{2}$ irradiation induced refractive index modulation may have non-uniform 
azimuthal distributions. As the laser exposure time decreases, a non-uniform azimuthal refractive index modulation becomes more likely due to the non equilibrium heat distribution within the short heating time period. Second, the critical fiber parameters, such as the diameter and the refractive indices of the core and cladding, in commercial products may vary from time to time. As a result, the simulation may not be as accurate as one expects.

\section{Conclusions}

In this paper, we demonstrated a new method to measure the $\mathrm{CO}_{2}$ laser irradiation-induced refractive index modulation in the core of a single mode optical fiber for the purpose of design and fabrication of LPFGs with desired transmission characteristics. With the assumption of a Gaussian-shaped axial refractive index perturbation, the peak refractive index change was measured using an optical fiber Fabry-Perot interferometer. With an $8 \mathrm{~W}$ laser power, $180 \mu \mathrm{m}$ beam width and $125 \mathrm{~ms}$ exposure time, the $\mathrm{CO}_{2}$ laser irradiation generated a negative refractive index change in the fiber core with a peak value of $-7.12 \times 10^{-4}$. The laser induced refractive index change was also measured at four different laser exposure times including 50, 75, 100 and 125

ms. Using the first two terms of Fourier series approximation of the periodic Gaussian-shaped index perturbation profile, the axial refractive index modulations were computed to simulate the LPFG transmission spectra. LPFGs with the same laser exposure parameters were also fabricated for the purpose of verification. Using a slightly modified core-cladding refractive index contrast, the simulated spectra matched well with those of real devices. It was also found that the refractive index was a sensitive function of the laser exposure time following almost a linear relation. This relation can be used to guide the selection of a preferred refractive index modulation, through accurate control of the laser exposure time, for fabrication of LPFGs with 
desired performance.

\subsubsection{High order mode long-period fiber grating refractive index sensor based on intensity}

\section{measurement}

\section{Theory and simulations}

Couple mode theory

Couple mode theory is a powerful tool for simulating LPFG structure. There are three steps in the grating spectra simulation process. The first step is to calculate the propagation constants of different LP modes. It is based on scalar approximation analysis to obtain the LP mode of a cylindrical dielectric waveguide. Fiber core, fiber cladding and infinite surrounding refractive index medium are considered as three layers of cylindrical waveguide.

The transverse electrical field component propagating along $\mathrm{z}$-axis is given as:

$$
\begin{aligned}
& \mathrm{U}_{0 \mathrm{j}, \mathrm{i}}(\mathrm{r}, \phi, \mathrm{z})=\exp \left(-j \beta_{0 j} z\right) \psi_{0 j, i}(r, \phi)=\exp \left(-j \beta_{0 j} z\right) \Phi(\phi) R_{0 j, i}(r) \\
& =\exp \left(-j \beta_{0 j} z\right) \times\left\{\begin{array}{l}
A_{0 j, i} J_{0}\left(r \gamma_{0 j, i}\right)+B_{0 j, i} Y_{0}\left(r \gamma_{0 j, i}\right) \\
A_{0 j, i} I_{0}\left(r \gamma_{0 j, i}\right)+B_{0 j, i} K_{0}\left(r \gamma_{0 j, i}\right)
\end{array}\right\} \text { when }\left(\begin{array}{c}
\beta_{0 j}\left\langle k_{0} n_{i}\right. \\
\left.\beta_{0 j}\right\rangle k_{0} n_{i}
\end{array}\right)
\end{aligned}
$$

where $\beta_{0 \mathrm{j}}$ is the propagation constant of $\mathrm{LP}_{0 \mathrm{j}}$ mode, $\mathrm{R}(\mathrm{r})$ is the radial variation of the modal field, $\gamma_{0 j, i}=\sqrt{\left|k_{0}^{2} n_{i}^{2}-\beta_{0 j}^{2}\right|}$ is the magnitude of the transverse wavenumber, $\phi$ is the azimutal angle, and $\mathrm{A}_{0 \mathrm{j}, \mathrm{i}}$ and $\mathrm{B}_{0 \mathrm{j}, \mathrm{i}}$ are normalized filed expansion coefficients determined by the boundary conditions within each layer i. $\mathrm{J}_{0}$ and $\mathrm{Y}_{0}$ are the ordinary Bessel functions of first and second kind of order 0 , while $\mathrm{I}_{0}$ and $\mathrm{K}_{0}$ are the modified Bessel functions of first and second kind of order 0 .

The fiber core and cladding refractive indexes are computed based on sellmeier's equation. The scalar mode theory and transfer matrix method are applied to calculate the propagation 
constants of the three layer cylindrical waveguide structure. During this process, the transverse electromagnetic field distributions of LP modes can also be obtained.

$$
P_{0 j}=\frac{\beta_{0 j}}{2 \omega \mu_{0}} \int_{\phi=0}^{2 \pi} d \phi \int_{r=0}^{r_{1}} R_{0 j}^{2}(r) r d r
$$

After that, the coupling coefficients between modes are computed by combining grating structure permittivity variation and transverse EM fields into the $2 \mathrm{D}$ integration. Because the longitudinal coupling coefficients are too small compared with transverse coupling coefficients for fiber modes, the transverse mode is just chosen for computation.

$$
K_{v j, u k}=\frac{\omega}{4 P_{0}} \times \int_{\phi=0}^{2 \pi} \int_{r=0}^{\infty} \Delta \varepsilon(r, \phi, z) \psi_{v j}(r, \varphi) \psi_{u k}(r, \varphi) r d r d \phi
$$

where $\psi(\mathrm{r}, \phi)$ is the transverse filed for an LP mode and $\Delta \varepsilon(r, \phi, z)$ is the permittivity variation which is expressed as:

$$
\begin{aligned}
& \Delta \varepsilon(r, z) \approx 2 \varepsilon_{0} n_{0}(r) \Delta n(r, z)=2 \varepsilon_{0} n_{0}(r) p(r) \sigma(z) S(z) \\
& =2 \varepsilon_{0} n_{0}(r) p(r) \sigma(z)\left[s_{0}+s_{1} \cos ((2 \pi / \Lambda) z)\right]
\end{aligned}
$$

where $\varepsilon_{0}$ is the free space permittivity, $n_{0}(r)$ is the refractive index profile of the fiber structure, and $\Delta n(r, z)$ is the variation of the refractive index. $p(r)$ is the transverse refractive index perturbation, $\sigma(z)$ is the optional apodization factor, and $S(z)$ is the longitudinal refractive index perturbation factor with $s_{0}$ and $s_{1}$ as the Fourier series of two terms, and $\Lambda$ is the grating period.

Bring the equation (4) into equation (3), the coupling coefficients will be expressed as:

$$
K_{v j, u k}=\sigma(z)\left[s_{0}+s_{1} \cos ((2 \pi / \Lambda) z)\right] \frac{\omega \varepsilon_{0}}{2 P_{0}} \times \int_{\phi=0}^{2 \pi} \int_{r=0}^{\infty} n_{0}(r) p(r) \psi_{v j}(r, \varphi) \psi_{u k}(r, \varphi) r d r d \phi
$$

Once the propagation constants and coupling coefficients are deduced, the fiber grating 
spectra can be simulated by solving the introduced couple mode theory, in which the interaction between optical modes is proportional to the coupling coefficient.

The generalized coupled mode equation is described as:

$\frac{d F_{0 k}(z)}{d z}=-j \sum_{j=1}^{M} K_{0 j, 0 k} F_{0 j}(z) \exp \left(-j\left(\beta_{0 j}-\beta_{0 k}\right) z\right) \quad$ for $k=1,2, \ldots M$

where $F_{0 j}$ is the normalized amplitude of $j$ mode.

\section{Phase matching curve}

After the calculation of core mode and cladding modes propagation constants, the central wavelengths of the attenuation bands of LPFG can be determined simply by modified first order Bragg condition.

$$
\left(\beta_{01}(\lambda)+s_{0} \zeta_{01,01}(\lambda)\right)-\left(\beta_{v j}(\lambda)+s_{0} \zeta_{0 j, 0 j}(\lambda)\right)=\frac{2 \pi}{\Lambda}
$$

where $\zeta$ is the coupling constant which is simplified from equation (5):

$$
\zeta=\frac{\omega \varepsilon_{0}}{2 P_{0}} \times \int_{\phi=0}^{2 \pi} \int_{r=0}^{\infty} n_{0}(r) p(r) \psi_{v j}(r, \varphi) \psi_{u k}(r, \varphi) r d r d \phi
$$

The modified first order Bragg condition is induced to decrease the prediction error considering the perturbation from the core mode and cladding mode self coupling. Based on the phase matching condition, a graph called as phase matching curve (PMC) can be plotted; this graph can indicate the relationship between fiber grating period and coupling wavelength.

The phase matching curve can be applied to help to choose the cladding mode corresponding to the specific grating period. Also, this graph can easily explain and predict the LPFG performance for various sensing application Patrick investigated the grating sensor response to surrounding refractive index (RI) variation. The resonance wavelength shift can be predicted 
very well in lower cladding mode, and the resonance wavelengths are determined by the intersection points between the phase matching curves and grating period lines. As the refractive index changes, the effective index of cladding mode is modified. The coupling coefficient is also changed. Thus, the new modified PMC will be plotted to interact with the grating period lines to calculate the shift of resonance wavelength.

But for TP LPFG, the sensing manner is a total different story. There are three stages in the interaction process between PMC and grating period straight line. Two points, one point or no point will occur during this process. Two resonance wavelengths appear as the interaction points are two, and one big resonance band appears as the interaction point is one. If there is no intersection point, it does not mean there is no mode coupling, for the operation near the turnaround-point area, the coupling still exists and the coupling strength is strongly related to the distance between the grating period line and PMC. If the distance is very large, there will be no resonance coupling, but there is a large region that can be coupled. In this case, the sensor performance cannot directly be predicted from PMC, the couple mode theory should be applied.

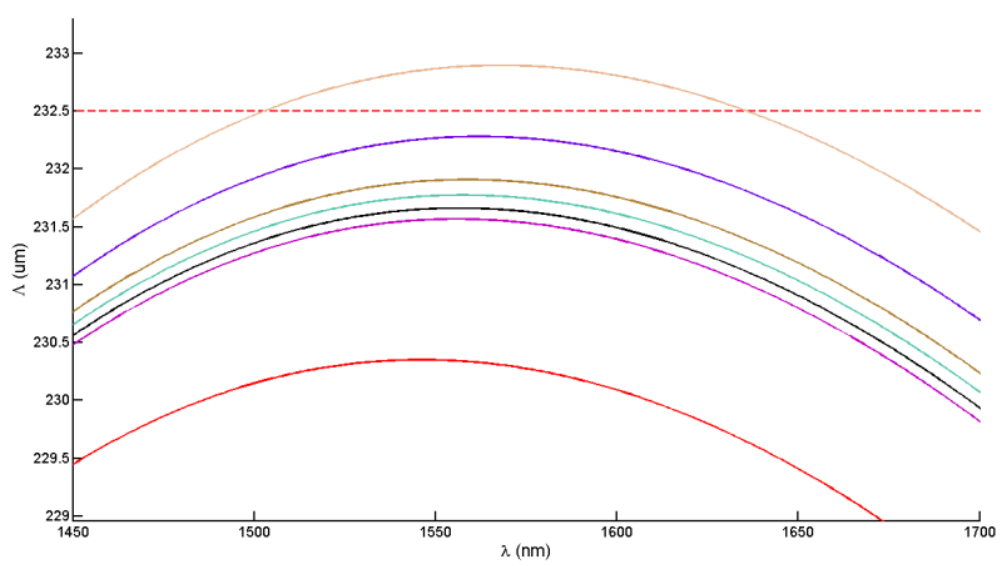

Figure 20 Phase matching curve for studied LP10 mode in corning SMF during various surrounding refractive index environments. 
Phase matching curves at different surrounding refractive index solutions are plotted in Figure 20. The fiber we used is corning SMF28e single mode fiber. The refractive index of fiber core and cladding are computed based on sellmeier's equation. We assume the index modulation parameter as $\mathrm{s} 0=\mathrm{s} 1=2.4 \times 10^{-4}$. The refractive index range is from $1 \sim 1.38$. The grating period is chosen as $232.5 \mu \mathrm{m}$.

As shown in Figure 20, the initial modified phase match curve in air is plotted as the red line. In that case, the phase match curve departs from the period a lot and there is no coupling from fiber core mode to $\mathrm{LP}_{0,10}$ mode. As the surrounding refractive index increases, the phase matching curve moves upwards, although the phase match curve and grating period line do not interact, there is already mode coupling between the core mode and cladding mode, at the refractive index equals to water, there is about $2 \mathrm{~dB}$ resonance dip shown up from the coupling mode simulation even there is still a gap between the matching curve (purple curve) and period line. Eventually, with the increase of RI value, PMC and period line will intersect each other, and the critical coupling happens, after that, two intersection points will occur and follow with two resonance wavelengths.

\section{Mode coupling simulation result}

Based on couple mode theory, the spectra evolution of TP LPFG can be simulated. Figure 21 (top) shows the simulation grating spectra evolution of $\mathrm{LP}_{0,10}$ mode with the surrounding $\mathrm{RI}$ change in $\mathrm{dB}$ range. From the beginning, there is no coupling happened, as the RI increase, the mode couple begin to increase and finally two resonance dips show up. To study the relationship between transmission strength and different RI value, the normalized transmission power trend in terms of different RI values is plotted in Figure 21 (bottom left). From this Figure, we can 
find there is linear like range from 0.6 to 0.1. To make it clear, Figure 21 (bottom right) shows the linear part of the sensor. This phenomenon makes the TP LPFG a good candidate as intensity based refractive index sensor, moreover, the linearity can help to simplify the signal modulation.
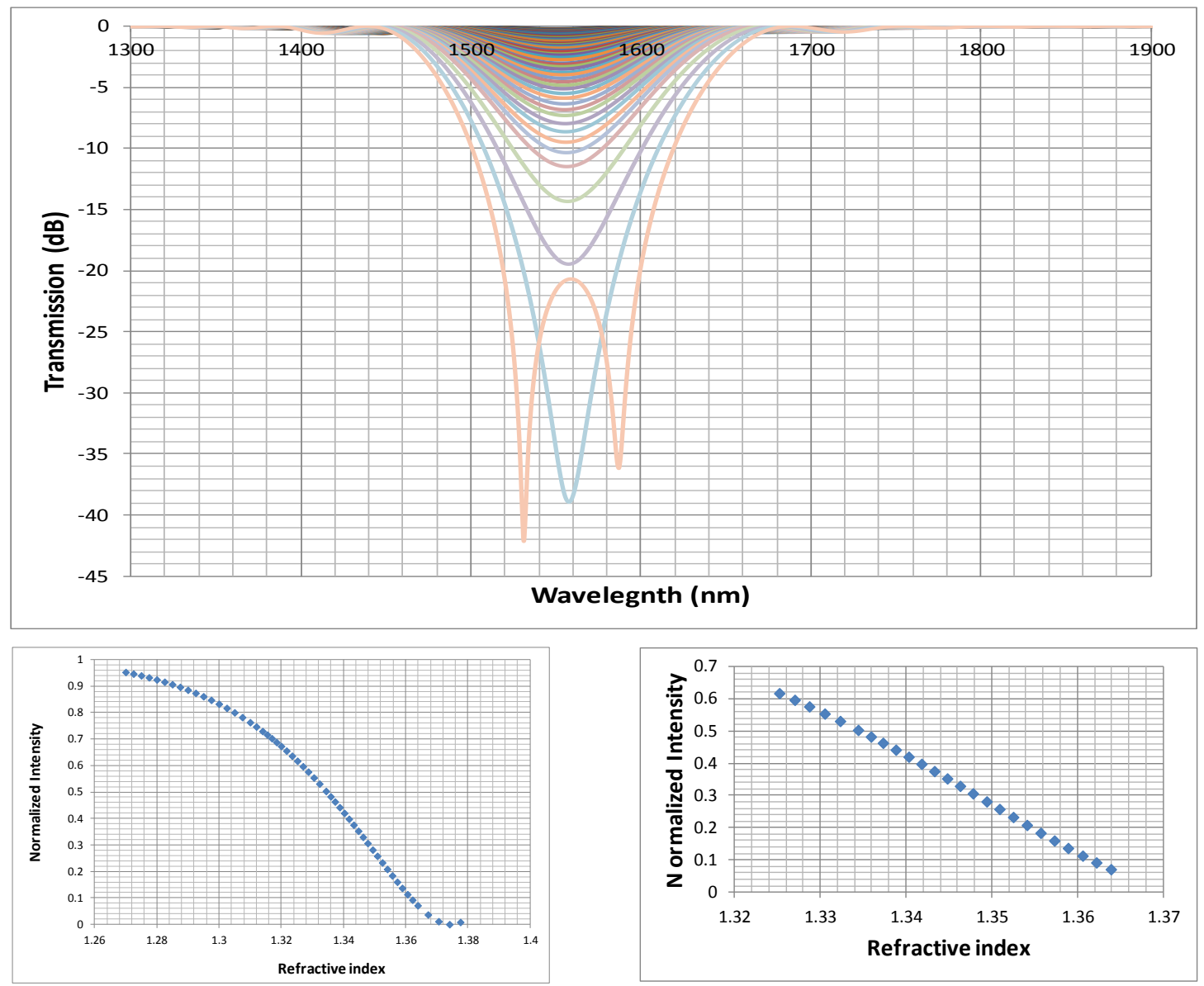

Figure 21 The grating spectra evolution of LP010 mode in respond to surrounding refractive index change (1.27 1.38) (top); the transmission power (TP LPFG) curve at different RI liquids (bottom left); the transmission power linear part at spectral RI range (bottom right)

\section{Experiment results}

By controlling the grating period and $\mathrm{CO}_{2}$ laser power, high order mode LPFG can be precisely fabricated to work at the turn around point for sensing application. The TP LPFGs are 
fabricated by point by point $\mathrm{CO}_{2}$ laser irradiation method.

The tested grating was fabricated using period $230 \mu \mathrm{m}$. The laser power is $6.8 \mathrm{~W}$, exposure time is $100 \mathrm{~ms}$, and the grating period for critical coupling at turning point is $227.5 \mu \mathrm{m}$. The refractive index solution is prepared by diluting sucrose crystal into de-ionized water; different concentration has its individual RI value. Figure 22 plots the evolution of grating spectra in different concentration of sucrose solutions in $\mathrm{dB}$ range. The inset picture plots the intensity value response to different RI in normalized unit. As shown in this Figure, there is linearity between the intensity values to refractive index at a range from 1.3333 to 1.37 . The experiment results correspond to simulation results very well. 

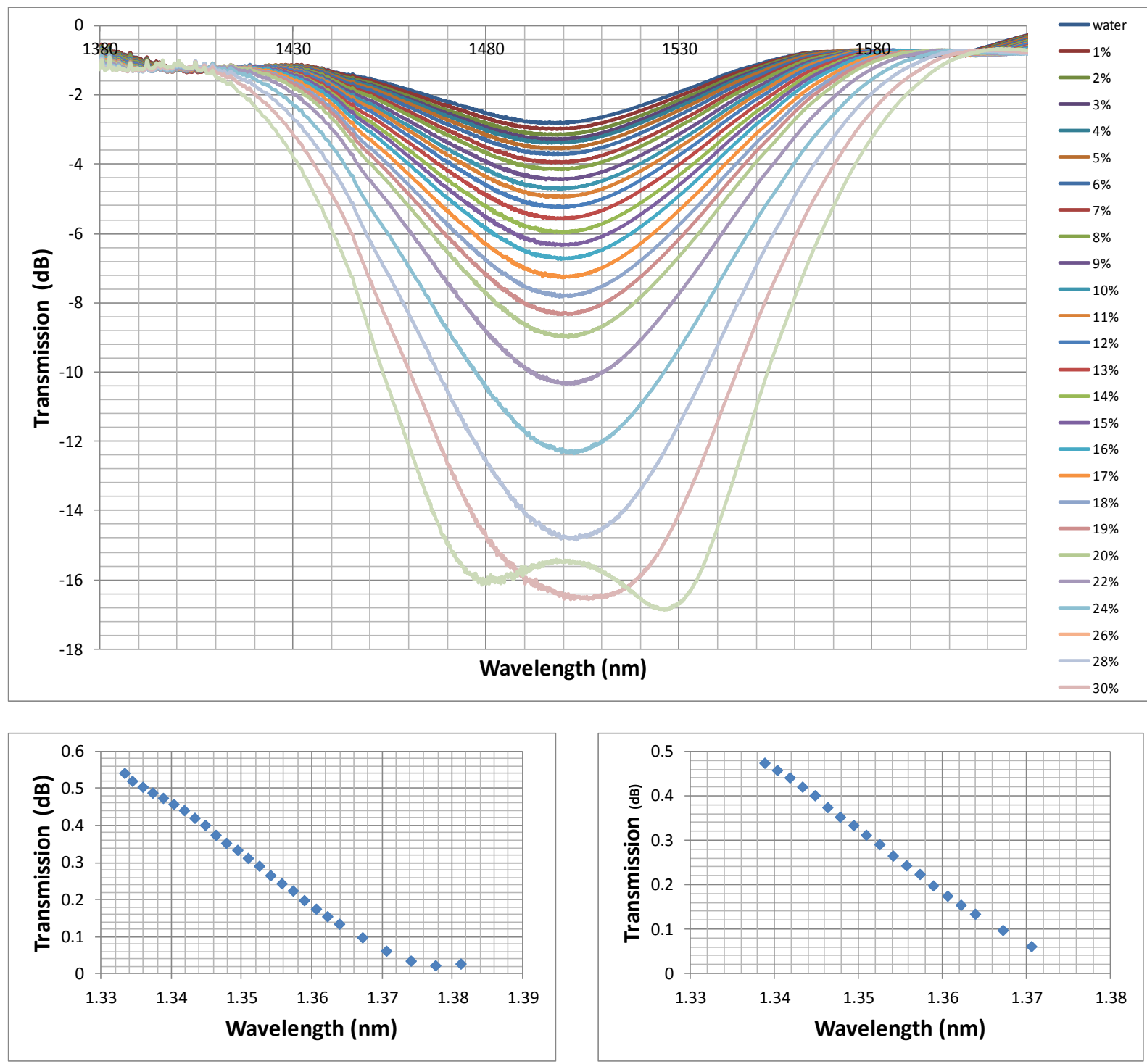

Figure 22 The grating spectra evolution of LP010 mode in respond to different concentration of sucrose solution (0-30\%) in experiment with grating period $\Lambda=230 \mu \mathrm{m}$ (top); the transmission power curve at different RI liquids (bottom left); and the transmission power linear trend at specific range (bottom right).

Due to the linear response, this kind of sensor can be demodulated very easily. Compared with nonlinear resonance wavelength shift of normal LPFG, the demodulation process is much easier in intensity based measurement. Once one gets two points of value of established RI value, 
he can deduce the other value from easy linear line approximation.

Glucose concentration is also tested with the intensity based LPFG in our lab. Using this kind of sensor, $0-40 \mathrm{mM}$ glucose concentration can be detected with high signal to noise ratio. Further research is still going on.

\section{Summary and conclusion}

Intensity based long period fiber grating has been studied by theoretical simulation and experimental RI liquids detection. There exists linear like range for RI detection. Compared with wavelength shift based LPFG measurement, this intensity based new method provides a fast, simple and cost effective solution for the RI detection, which can provide potential application in chemical and bio-sensing areas.

\subsubsection{Multiplexed sensor system design and instrumentation}

Based on the evaluation results of sensor devices, we have designed and implemented two sensor characterization systems using commercially available instruments. Control software and signal processing algorithms have been fully developed to process the LPFG sensor signals. The algorithms were based on the mathematic models of the nanomaterial-coated gratings. One system is placed at the Missouri University of Science and Technology, and the other at the University Cincinnati. These two systems are currently used for testing sensors.

The typical spectral width of the gratings was about $15 \mathrm{~nm}$. In the entire spectral range of the tunable laser (1510-1640nm), we were able to multiplexing 9 gratings. These gratings were multiplexed in a single fiber and interrogated by a measurement system for simultaneous measurement of multiple parameters. 


\subsection{Fiber Inline Core-Cladding-Mode Mach-Zehnder Interferometer Fabricated by Two-Point CO2 Laser Irradiations}

\subsubsection{Device Principle}

Figure 23(a) shows the structural diagram of a fiber inline MZ type CCMI, made by two consecutive $\mathrm{CO}_{2}$ laser irradiations on a single mode fiber, separated by a distance of $\mathrm{L}$. The $\mathrm{CO}_{2}$ laser irradiation induces a microbend on the fiber with its microscopic image shown in Fig. b. The first microbend couples part of the light guided by the fiber core into the cladding. Passing through the interferometer section, the light traveling inside cladding is coupled back to the core at the second laser irradiation induced microbend, mixing with the core mode component to generate an interference signal.

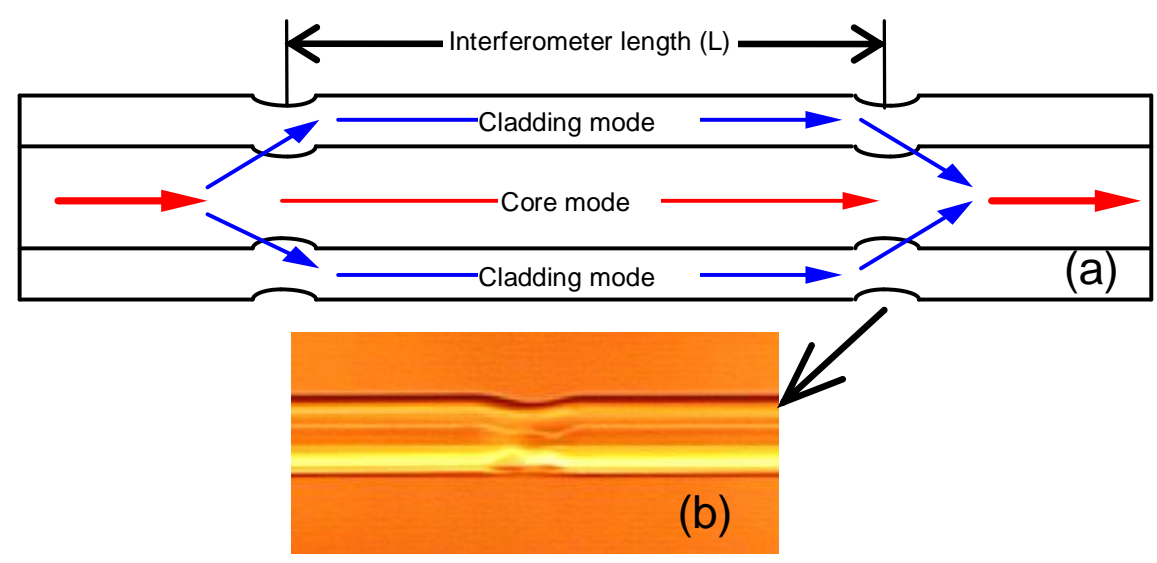

Figure 23 Fiber inline core-cladding-mode MZI fabricated by 2-point $\mathrm{CO} 2$ laser irradiation: (a) schematic diagram; (b) microscopic image of laser induced micronotch; (c) illustration of the interference spectrum. 
The device can be modeled using two-beam interference equation:

$$
I=I_{1}+I_{2}+2 \sqrt{I_{1} I_{2}} \cos \left[\frac{2 \pi\left(n_{c}^{e f f}-n_{c l}^{e f f}\right) L}{\lambda}\right]
$$

where, $I$ is the intensity of the interference signal; $I_{1}$ and $I_{2}$ are the intensity of light propagating in the fiber core and cladding, respectively; $L$ is the interferometer length; $\lambda$ is the free space wavelength; $\mathrm{n}_{\mathrm{c}}^{\text {eff }}$ and $\mathrm{n}_{\mathrm{cl}}^{\text {eff }}$ are the effective refractive indices of the core and cladding, respectively. The effective refractive index $\left(n^{\text {eff }}\right)$ is related to the propagation constant $(\beta)$ by $\beta=2 \pi n^{\text {eff }} / \lambda$.

According to Equation (1), the interference signal reaches its minimum $\left(\mathrm{I}_{\min }\right)$ when the phase of the cosine term becomes an odd number of $\pi$. That is

$$
I=I_{\min }, \quad \text { when } \quad \frac{2 \pi\left(n_{c}^{e f f}-n_{c l}^{e f f}\right) L}{\lambda_{v}}=(2 k+1) \pi
$$

where $\mathrm{k}$ is an integer and $\lambda_{\mathrm{v}}$ is the center wavelength of the interference valley of $\mathrm{k}$-th order.

The effective index of the cladding mode $\left(\mathrm{n}_{\mathrm{cl}}{ }^{\text {eff }}\right)$ is a function of the refractive index of the surrounding medium. On the other hand, the core mode is shielded by the cladding layer so its effective index does not vary with the ambinet index change. In Equation (2), taking the derivative of $\mathrm{n}_{\mathrm{cl}}^{\text {eff }}$ with respect to $\lambda_{\mathrm{v}}$, one finds:

$$
\frac{d n_{c l}^{e f f}}{d \lambda_{v}}=-\frac{(2 k+1) \pi}{2 \pi L}=-\frac{n_{c}^{e f f}-n_{c l}^{e f f}}{\lambda_{v}} .
$$

The interferometer can thus be used as a sensor to monitor the environmental refractive index change based on the wavelength shift of a particular interference valley. It is worth noting that the sensitivity of the interferometer is independent on its length. Equation (3) is also applicable to other characteristic spectral positions such as the interference peak and the center point of the interferogram. In addition, curve fitting of the interference fringe can also improve the 
measurement accuracy.

\subsubsection{Experiments, Results and Discussion}

$\mathrm{A} \mathrm{CO}_{2}$ laser (SYNRAD, Inc.) with a free space wavelength of $10.6 \mu \mathrm{m}$ was used to fabricate the sensor. A ZnSe cylindrical lens with a focal length of $50 \mathrm{~mm}$ was used to shape the $\mathrm{CO}_{2}$ laser beam into a narrow line with a linewidth of around $220 \mu \mathrm{m}$. The $\mathrm{CO}_{2}$ laser was controlled by a computer so that the output power and exposure duration could be accurately adjusted. A three dimensional (3D) motorized translation stage was used to position the singlemode optical fiber (Corning SMF-28e) to the center of the laser beam with the fiber axis perpendicular to the laser line. During fabrication, the $\mathrm{CO}_{2}$ laser heats the fiber and created the first microbend. Controlled by the computer, the translation stage moved the fiber with a certain distance. Then the laser was fired again to create the second microbend.

Using the described system, we made several fiber inline MZ type CCMIs with different lengths. The laser power was set to $10 \mathrm{~W}$ and the exposure duration was $300 \mathrm{~ms}$. Figure 24 shows the interference spectra of MZ-CCMIs with the length of 5, 10, 20 and 40mm, respectively. The typical interference fringe had a visibility around $20 \mathrm{~dB}$, which was sufficient for most sensing applications. The background loss varied among devices with a typical value around $12 \mathrm{~dB}$. With the increase of the interferometer length, the spectral separation between two adjacent interference fringes decreased in approximately an inversely proportional fashion. 

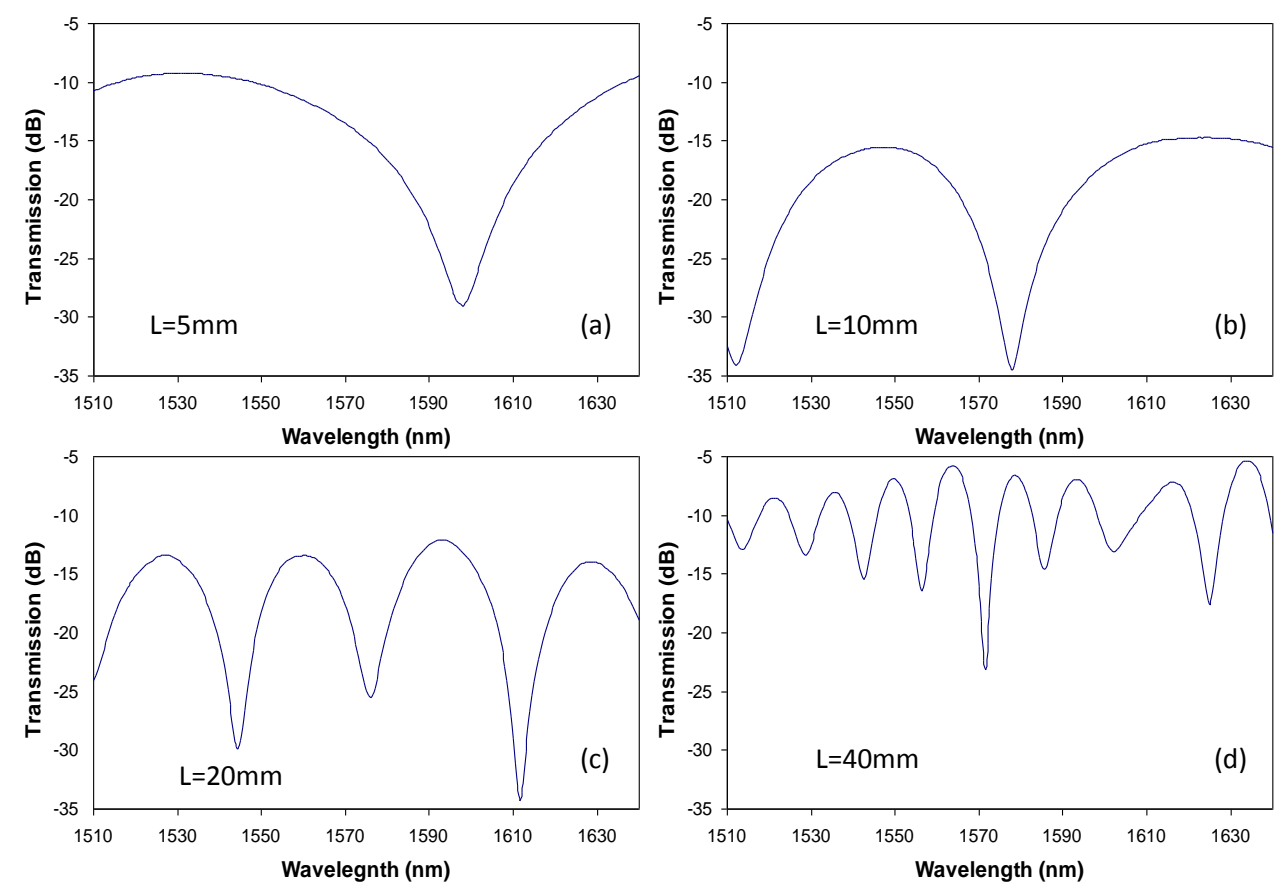

Figure 24 Transmission spectra of fiber inline core-cladding-mode MZIs with different interferometer lengths: (a) $5 \mathrm{~mm}$; (b) $10 \mathrm{~mm}$; (c) $20 \mathrm{~mm}$; (d) $40 \mathrm{~mm}$.

The MZ-CCMI with a length of $5 \mathrm{~mm}$ was employed to evaluate its sensitivity towards temperature variation. The interferometer was placed in a quartz tube without stress, fixed in a tubular electric furnace (Lindberg/blue M). The device was first heated up to $120^{\circ} \mathrm{C}$ read from the built-in thermometer of the furnace. The furnace was then turned off to allow the temperature cool down while the interference spectrum was recorded at every $10^{\circ} \mathrm{C}$ of temperature dropping. The recorded interference spectra were processed to find the spectral position of the interference minima. The test ended till the temperature inside the furnace reached $50^{\circ} \mathrm{C}$. Fig. 3 shows the wavelength shift of the interference minimum as a function of the furnace temperature where the relation is roughly linear. Linear-fitting of the experiment data indicated that the temperature sensitivity of the device was $0.0817 \mathrm{~nm} /{ }^{\circ} \mathrm{C}$. The temperature sensitivity of the device can be attributed to the different thermo-optic dependence of the fiber core and cladding $[5,7] . \mathrm{n}_{\mathrm{c}}{ }^{\text {eff }}$ and 
$\mathrm{n}_{\mathrm{cl}}^{\text {eff }}$ changes differently when temperature varies, causing a phase shift of the interference fringe.

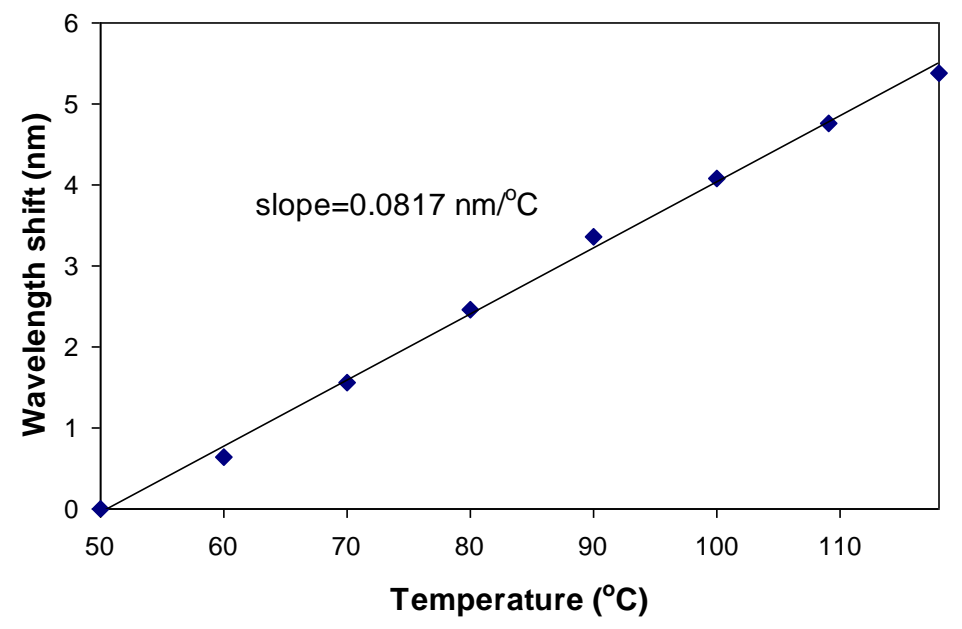

Figure 25 Temperature response of the fiber inline MZ-CCMI with a length of $5 \mathrm{~mm}$ fabricated by 2point $\mathrm{CO} 2$ laser irradiation.

The same MZ-CCMI was evaluated for its capability for refractive index measurement at room temperature. Various liquids were used in the tests, including deionized (DI) water, isopropanol (IPA) and refractive index liquids ranging from 1.40 to 1.44 . The device was fixed on a glass plate to avoid any bending induced signal change. We then immersed the glass plate into different liquids and recoded the interference fringes for each test. The device was carefully cleaned by subsequent acetone and water ultrasonic baths and air dried between tests to avoid any liquid residue from previous test.

Figure 26 shows the wavelength shift of the interferometer with respect to environmental refractive index change. The increase of ambient refractive index caused a shift of the interference spectrum towards the short wavelength. In addition, the sensitivity grew as the 
ambient index approaching that of the cladding. This can be explained by the well known waveguide theory that the effective index of the cladding mode increases nonlinearly with the index of the surrounding medium.

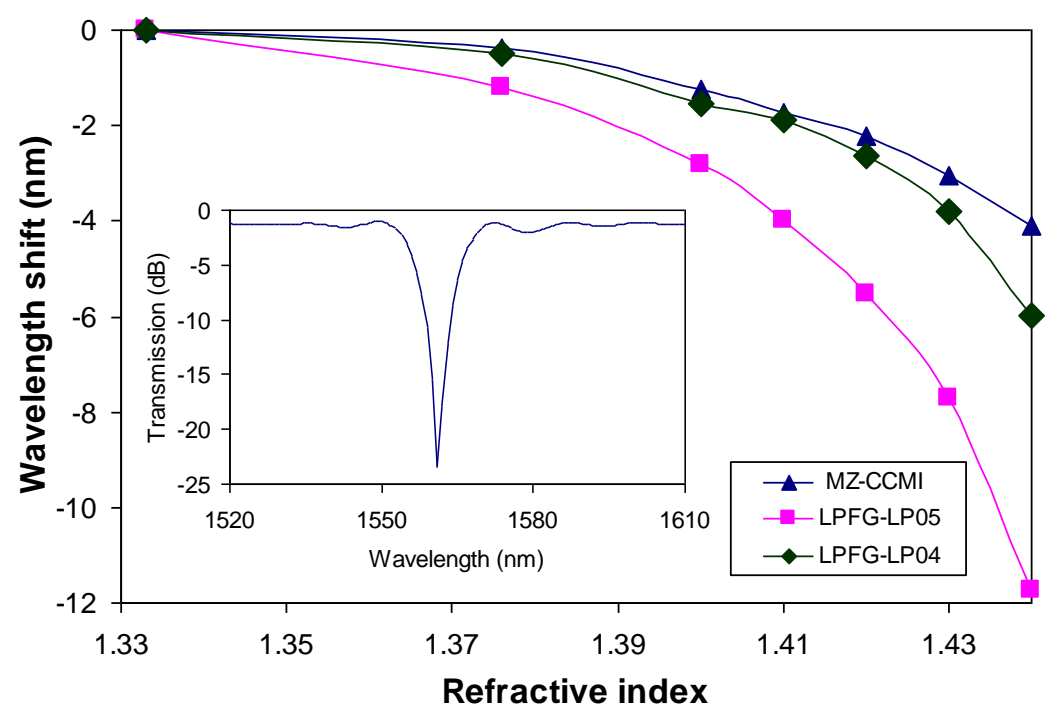

Figure 26 Wavelength shift as a function of refractive index in MZ-CCMI and LPFGs with LP04 and LP05 cladding modes (Inset: transmission spectrum of LPFG with LP05 cladding mode).

We also compared the device with LPFGs in terms of refractive index measurement sensitivity. Two LPFGs with $\mathrm{LP}_{04}$ and $\mathrm{LP}_{05}$ cladding modes were used for comparison. The LPFGs were fabricated by point-by-point $\mathrm{CO}_{2}$ laser irradiations. The LPFG-LP $\mathrm{L}_{04}$ had a period of $610 \mu \mathrm{m}$, length of $50 \mathrm{~mm}$, and resonance wavelength of $1570 \mathrm{~nm}$ in air at room temperature. The LPFG-LP $_{05}$ (with its transmission spectrum in air given in the insert of Figure 26) had a period of $525 \mu \mathrm{m}$, length of $50 \mathrm{~mm}$, and resonance wavelength of $1561 \mathrm{~nm}$. The shifts in resonance wavelength of LPFGs in response to the refractive index changes are shown in Figure 26 for comparison.

The resonance wavelength $\left(\lambda_{\text {res }}\right)$ of a LPFG can be roughly estimated by its phase matching 
condition $\lambda_{\text {res }}=\left(\mathrm{n}_{\mathrm{c}}{ }^{\text {eff }}-\mathrm{n}_{\mathrm{cl}}\right.$ eff $) \Lambda$, where $\Lambda$ is the period of the grating. Accordingly, the relative effective index change of the cladding mode $\left(\mathrm{dn}_{\mathrm{cl}}{ }^{\text {eff }}\right)$ is proportional to the relative resonance wavelength shift $\left(\mathrm{d} \lambda_{\text {res }}\right)$, given by:

$$
\frac{d n_{c l}^{\text {eff }}}{d \lambda_{\text {res }}}=\frac{n_{c}^{e f f}-n_{c l}^{\text {eff }}}{\lambda_{\text {res }}}
$$

Comparing Equations (3) and (4), one finds that a LPFG and a CCMI operate based on the same mechanism that the effective index of the cladding mode is a function of the ambient index. The higher the order of the cladding mode, the more sensitive to ambient index change. From Figure 26, we find that the sensitivity of the CCMI is smaller than LPFG-LP 04 and LPFG-LP 05 , but very close to $\mathrm{LPFG}-\mathrm{LP}_{04}$. This suggested that the order of the cladding mode excited in this CCMI was lower than but very close to $\mathrm{LP}_{04}$. It is possible for the laser exposure to excite more than one cladding mode. However, the high-quality interference fringes of different interferometer lengths indicated that one cladding mode dominated.

\subsubsection{Conclusion}

To summarize, we report a new method to fabricate fiber inline MZ type CCMI using 2-point $\mathrm{CO}_{2}$ laser irradiations. High quality interference spectra with fringe visibility of about $20 \mathrm{~dB}$ were obtained for four different lengths $(5,10,20$ and $40 \mathrm{~mm})$. The MZ-CCMI with a length of 5mm has a temperature sensitivity of $0.0817 \mathrm{~nm} /{ }^{\circ} \mathrm{C}$. The $\mathrm{CCMI}$ had a sensitivity for refractive index measurement smaller than but very close to that of a LPFG of $\mathrm{LP}_{04}$ cladding mode, indicating that the $\mathrm{CO}_{2}$ laser irradiation mainly promotes light coupling from the core mode to the low order cladding modes. The $\mathrm{CO}_{2}$ laser method had the advantage of fabricating CCMIs with short and precisely controlled length. The easy fabrication and simple structure make the device useful for monitoring refractive index changes. 


\subsection{Testing the performance of thermal LPFGs}

The thermal LPFGs were tested preliminarily in our lab to evaluate their temperature sensitivity, refractive index sensitivity, strain sensitivity, and high temperature stability and annealing effects. These data are important to optimize the fabrication process as well as to optimize the design of gas sensors.

\subsubsection{Temperature sensitivity}

The origin of the temperature sensitivity of LPFGs can be described by a differentiating equation.

$$
\frac{d \lambda}{d T}=\frac{d \lambda}{d\left(\delta n_{e f f}\right)}\left(\frac{d\left(\delta n_{e f f}\right)}{d T}-\frac{d\left(\delta n_{c l}\right)}{d T}\right)+\Lambda \frac{d \lambda}{d \Lambda} \frac{1}{L} \frac{d L}{d T}
$$

where $\lambda$ is the central wavelength of the attenuation band is the temperature, $n_{\text {eff }}$ is the effective refractive index of the core mode, $n_{c l}$ is the effective refractive index of the cladding mode, $\delta n_{\text {eff }}=\left(n_{\text {eff }}-n_{c l}\right), L$ is the length of the LPFG and $\Lambda$ is the period of the LPFG.

The first term on the right-hand side of equation (15) is the material contribution, and is related to the change in the differential refractive index of the core and cladding. This contribution is dependent upon the composition of the fiber and is dependent upon the order of the cladding mode. The material effect dominates for coupling to low order cladding modes. For coupling to higher-order cladding modes, the material effect can be negligible.

The second term is the waveguide contribution as it results from changes in the LPFG's period. The magnitude and sign of the term depend upon the order of the cladding mode. For 
coupling to low-order cladding modes $d \lambda / d \Lambda$ is positive, while for the higher-order cladding modes this term is negative.

A thermal LPFG was experimentally tested for its thermal dependence. The grating was placed in an electric furnace where a thermocouple was placed side by side with the LPFG. The transmission spectra of the LPFG at the temperatures of 90F, 300F, 400F, 500F, 600F, $700 \mathrm{~F}$, and 800F are shown in Figure 27. The experiment skipped 200F because the electric furnace was not stable when the set temperature was below 300F. The LPFG resonance wavelength shifted towards the long wavelength as the temperature increased, indicating that the grating mainly coupled the core mode to low-order cladding modes.

Figure 28 shows the resonance wavelength of the LPFG calculated at different temperatures. The resonance wavelength had an almost linear relation with temperature. This means that we can potentially design uncoated LPFGs to compensate for the temperature dependence of the gas sensor. 


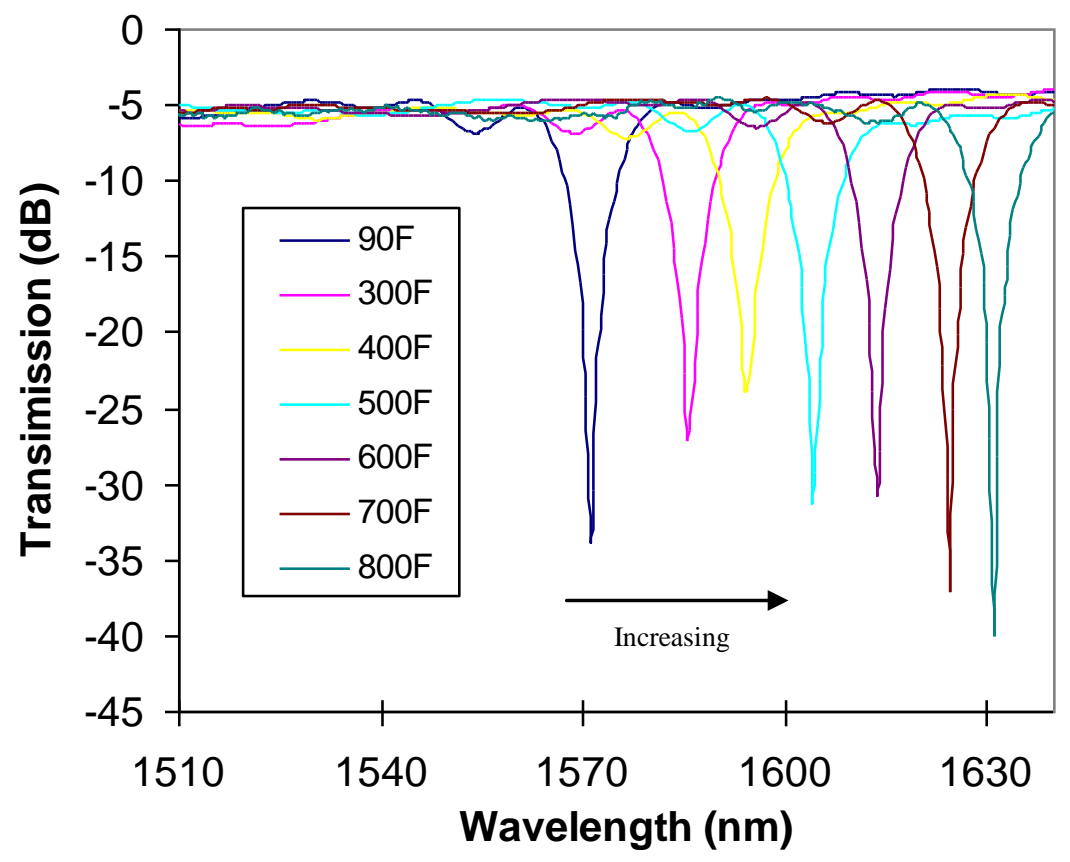

Figure 27 LPFG transmission spectrum at various ambient temperatures. The LPFG had a period of $500 \mu \mathrm{m}$ and was fabricated using Corning SMF-28 single mode fibers.

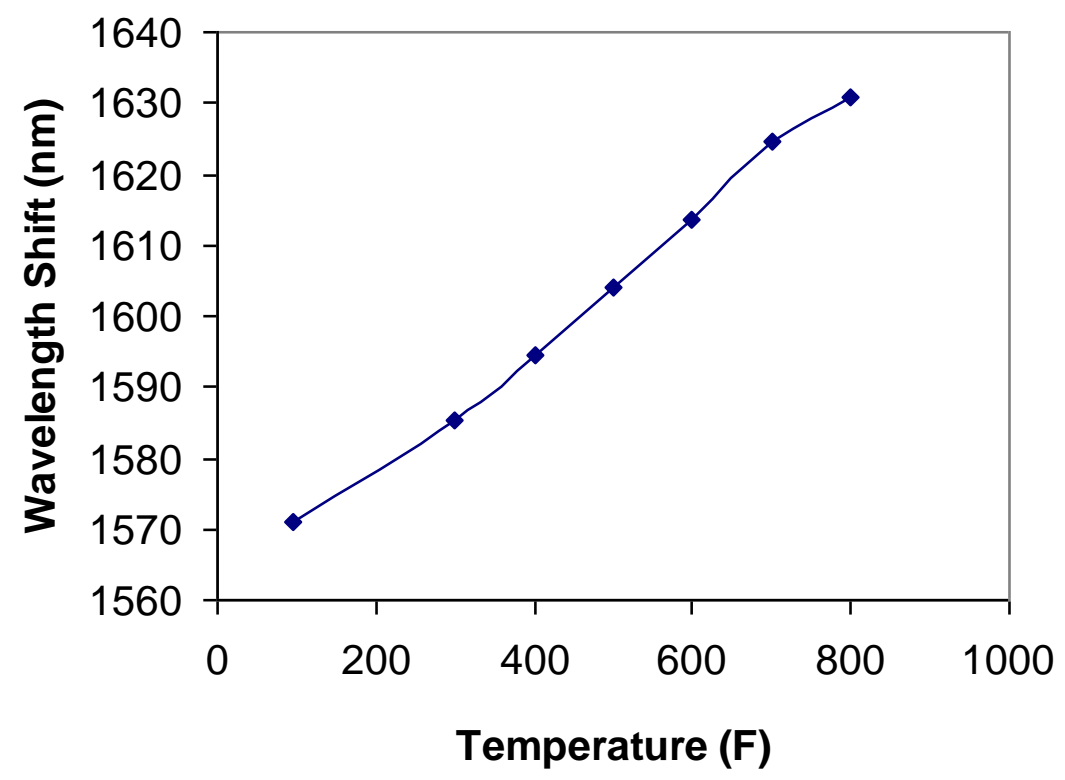

Figure 28 Shifts in the LPFG resonance wavelength as a function of temperature. 


\subsubsection{Refractive index sensitivity}

Because the phase matching condition are dependent upon the effective refractive indices of the cladding modes which are dependent upon the difference between the refractive index of the cladding and that of the medium surrounding the cladding, the central resonance wavelengths of the attenuation bands thus show a dependence upon the refractive index of the medium surrounding the cladding.

The sensitivity of the thermal LPFG resonance wavelength to the refractive index of the surrounding medium was preliminarily evaluated in our lab. The LPFG was immersed in air, water, and isopopanol solution and the spectrum was recorded as shown in Figure 29, where it is clear that the resonance wavelength shifts as the medium changed. As the refractive index of the surrounding medium increased, the resonance spectrum shift towards the short wavelength.

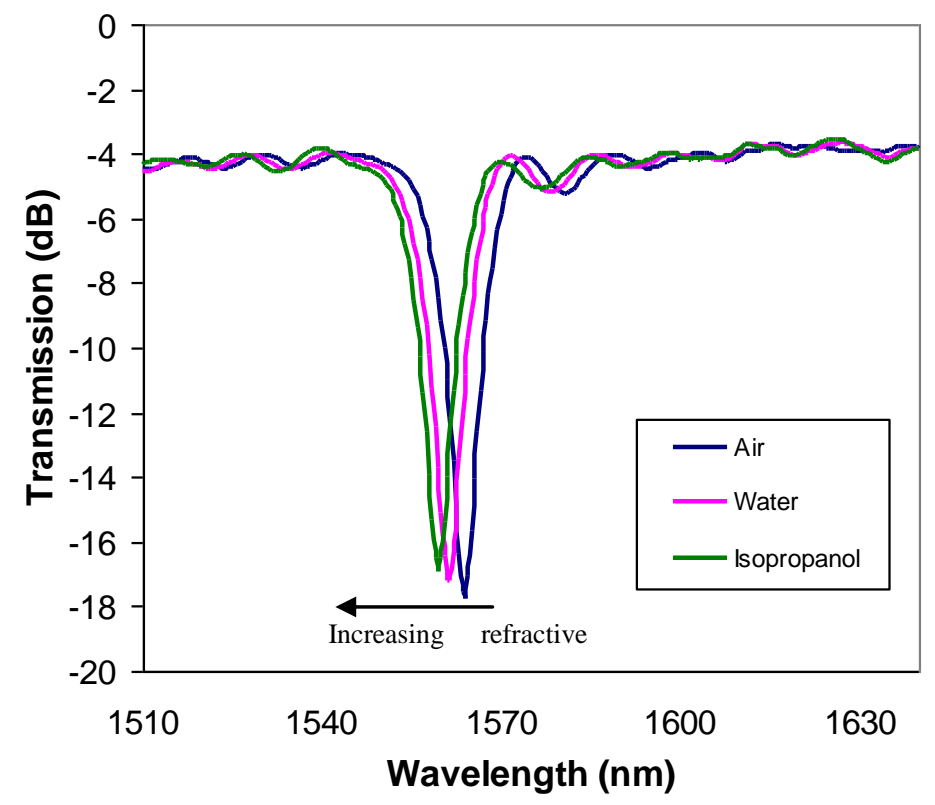

Figure 29 LPFG transmission spectrum in air, water, and isopropanol. The LPFG has a period of 515 $\mu \mathrm{m}$ and was fabricated using Corning SMF-28 single mode fibers. 


\subsubsection{Sensitivity to strain/fiber bending}

LPFGs are also sensitive to strain. The axial strain sensitivity of LPFGs may be described by the following differentiating equation

$$
\frac{d \lambda}{d \varepsilon}=\frac{d \lambda}{d\left(\delta n_{e f f}\right.}\left(\frac{d\left(\delta n_{e f f}\right)}{d \varepsilon}-\frac{d\left(\delta n_{c l}\right)}{d \varepsilon}\right)+\Lambda \frac{d \lambda}{d \Lambda}
$$

The sensitivity comprises material and waveguide effects, the material effects being the change in dimension of the fiber and the strain-optic effect, with the waveguide effects arising from the slope of the dispersion term $d \lambda / d \Lambda$. Appropriate choice of grating period and fiber composition will thus allow the generation of attenuation bands with positive, negative or zero sensitivity to strain.

As the fiber stretches or bends, the period of the LPFG changes correspondingly, causing the shift of the resonance wavelength. To verify this, a thermal LPFG was mounted on a steel plate so that it would bend following the deformation of the steel plate. Figure 30 shows the fiber bending effects on the LPFG transmission spectrum. It can be seen that the fiber bending shifted the resonance wavelength towards the long wavelength. It was also noticed that the shift in the resonance wavelength was accompanied by a reduction in the loss and a broadening of the spectral dropout. 


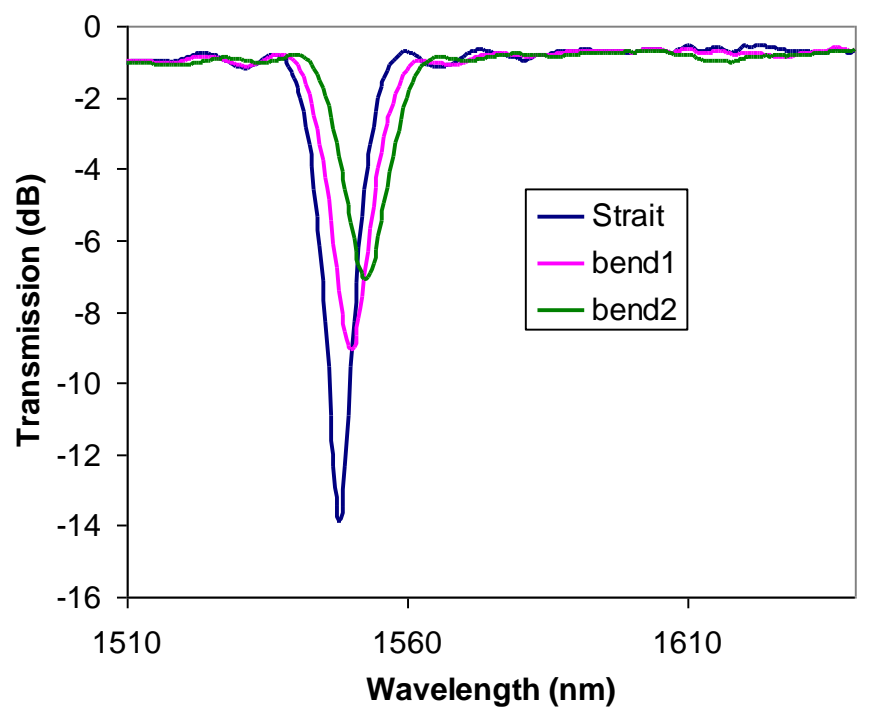

Figure 30 Shift of the resonance wavelength caused by bending. The LPFG has a period of $515 \mu \mathrm{m}$ and was fabricated using Corning SMF-28 single mode fibers.

\subsubsection{High-temperature stability and annealing effects}

The stability of LPFGs at high temperatures was investigated as they were designed to be used in high temperature environments. Thermally induced refractive index modulations intend to be decrease when the grating is subject to very high temperatures. This is mainly caused by the annealing process as the built-in thermal stresses intend to release under long time exposure to very high temperatures. The potential decrease of the refractive index modulation during the annealing process could be a major practical constraint in such applications.

The gratings' thermal stability was studied experimentally by placing the LPFG in high temperature environments. At temperatures below $500^{\circ} \mathrm{C}$, no obvious drift of the transmission spectrum was observed for the duration of 8 hours. However, as the temperature increased to $650^{\circ} \mathrm{C}$, the whole transmission spectrum started to drift towards the short wavelength as shown in Figure 31. The resonance wavelength as a function of the duration is plotted in Figure 32. 
Although there was about 9nm drift in the resonance wavelength drifted for the entire duration of 8 hours, it was encouraging to notice that the drifting slowed down as the exposure time increased. It was also found that the peaks position changed about $1 \mathrm{~nm}$ after cooling down to room temperature. This irreversible change was attributed to the stress relief and the plastic deformation of the fiber after annealing at such high temperatures. It was also worth noting that there was no obvious degradation of the LPFG quality after 8 hours exposure to $650^{\circ} \mathrm{C}$ because the LPFG attenuation band maintained the same strength and shape compared to what were before.

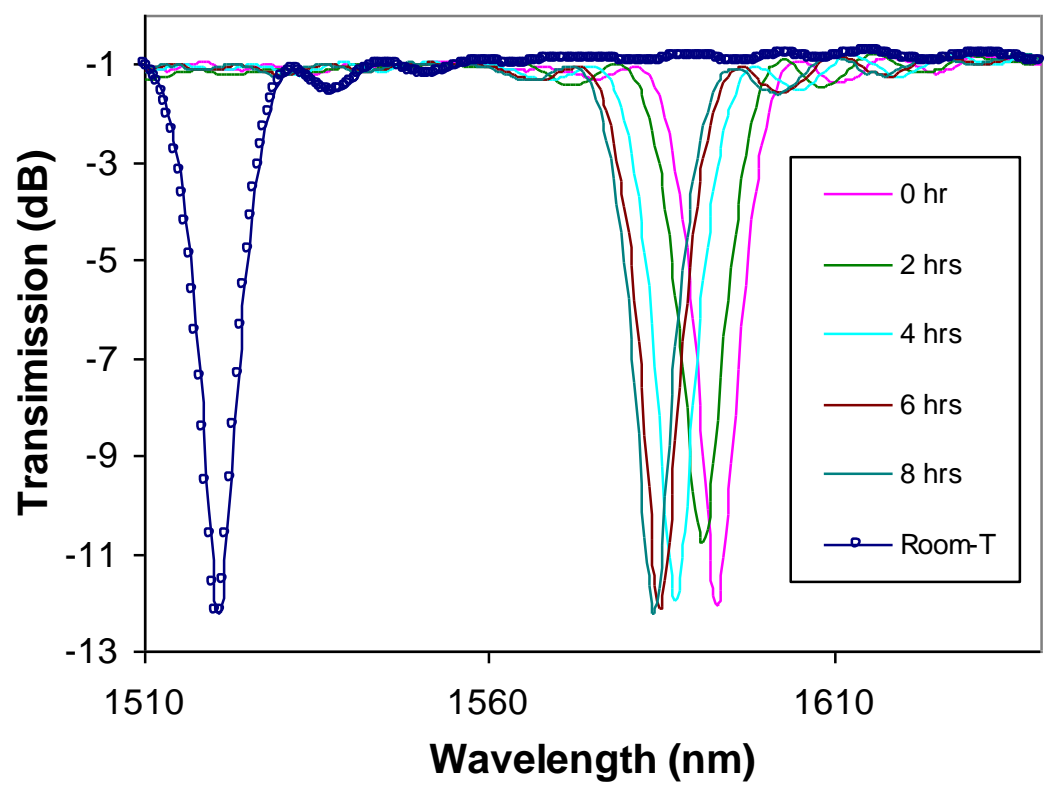

Figure 31 Shift of the LPFG transmission spectrum in response to annealing at $650^{\circ} \mathrm{C}$ for 8 hours. The LPFG had a period of $500 \mu \mathrm{m}$ and was fabricated using Corning SMF-28 single mode fibers. 


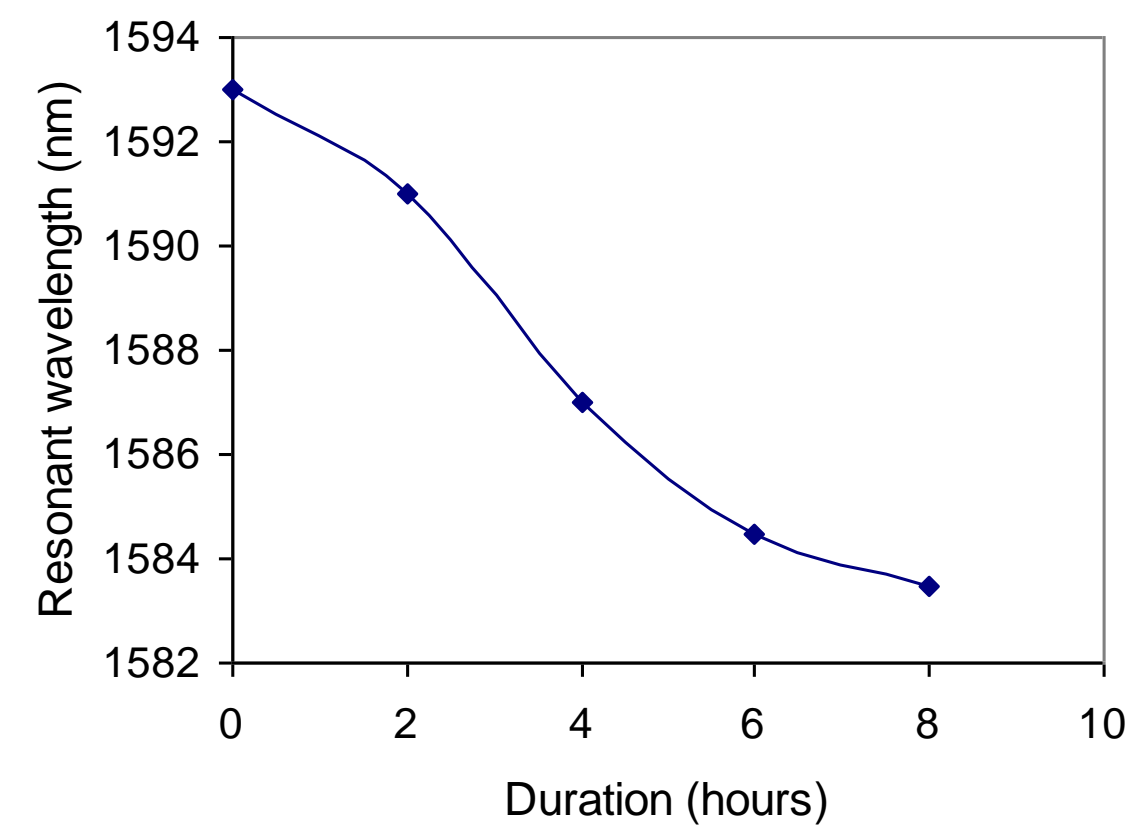

Figure 32 Drift of the LPFG resonance wavelength caused by annealing at $650^{\circ} \mathrm{C}$.

The experiment was repeated at $700^{\circ} \mathrm{C}$ for 8 hours. The transmission spectrum of the LPFG is shown in Figure 33. Figure 34 shows the LPFG resonance wavelength as a function of the duration of annealing at $700^{\circ} \mathrm{C}$. It can be seen that the resonance wavelength continued drift towards the short wavelength with the drifting rate decreased slightly after several hours annealing. However, this decrease in drift slop was not as obvious compared to the case of $650^{\circ} \mathrm{C}$. In addition to spectrum shift, there was a noticeable degradation in the strength of the resonance peak. As shown in Figure 35, the transmission of the LPFG resonance peak was decreased by $3 \mathrm{~dB}$ after 8 hours annealing at $700^{\circ} \mathrm{C}$. All these indicate that a higher operating temperature would have more severe effect on the LPFG stability. 


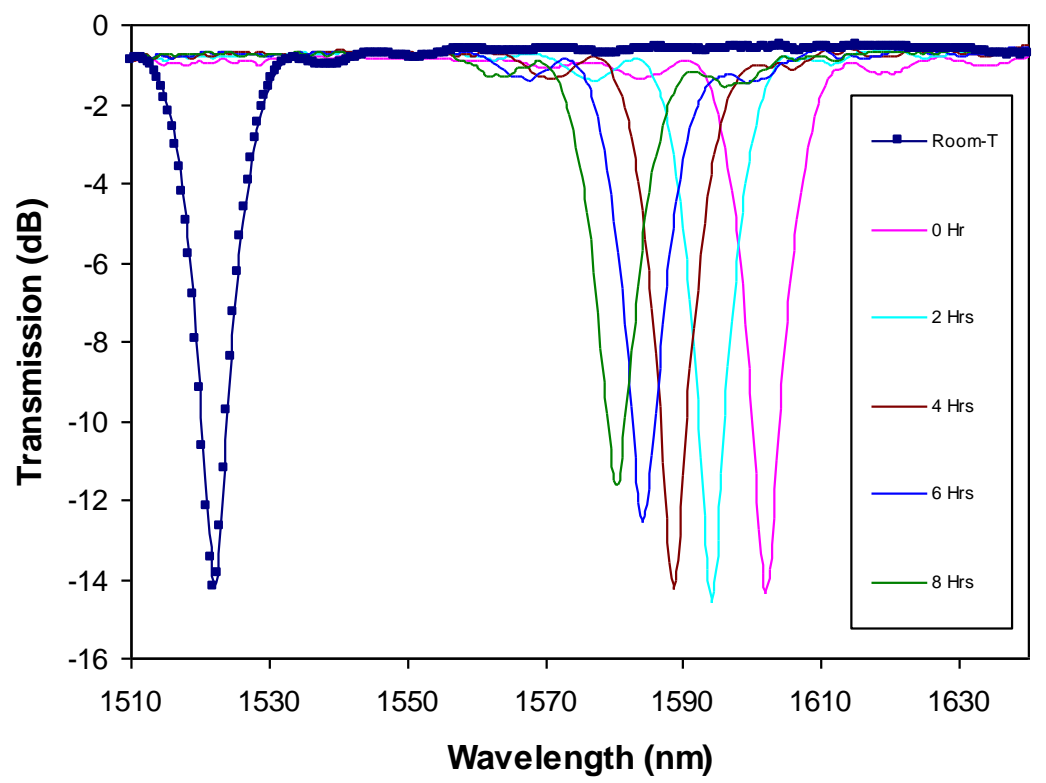

Figure 33 Shift of the LPFG transmission spectrum in response to annealing at $700^{\circ} \mathrm{C}$ for 30 hours. The LPFG had a period of $515 \mu \mathrm{m}$ and was fabricated using SMF-28 single mode fibers.

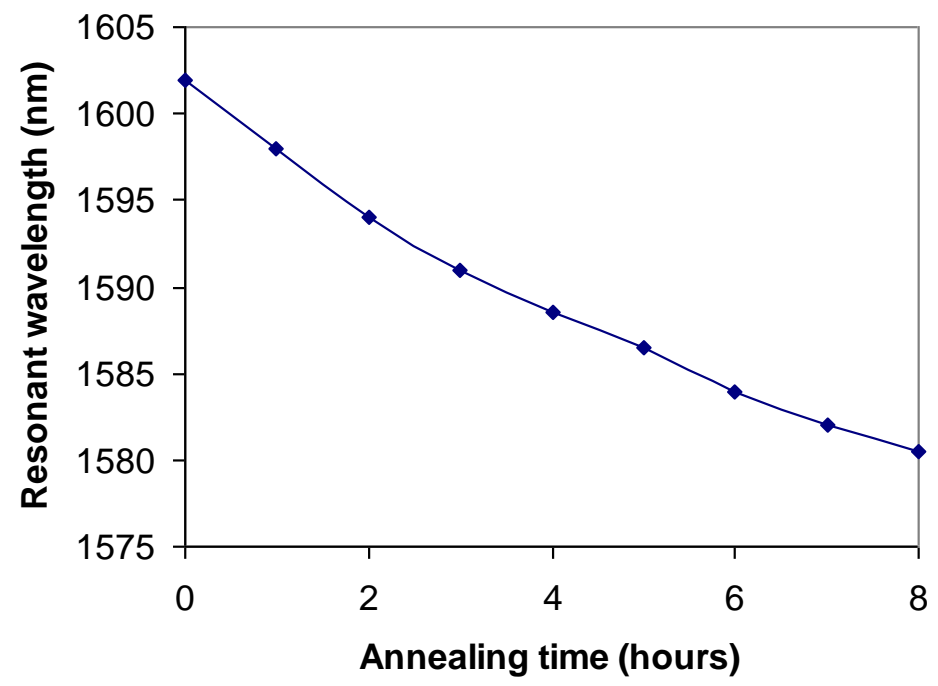

Figure $34 \mathrm{LPFG}$ resonance wavelength as a function of annealing time at $700^{\circ} \mathrm{C}$. 


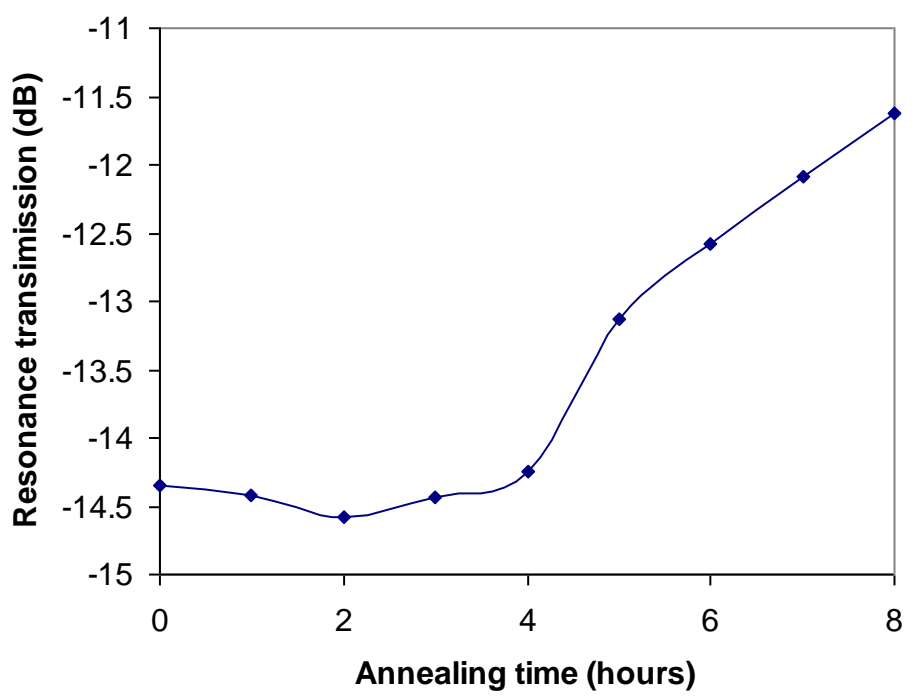

Figure 35 LPFG peak transmission as a function of annealing time at $700^{\circ} \mathrm{C}$.

\subsubsection{Long term thermal stability test and improvement.}

Experiments were also conducted at UMR to test the long term stability of the fabricated LPFGs. The first experiment was performing using as a LPFG fabricated using Corning SMF-28 singlemode fiber. The LPFG was hosted inside a $1 / 8$-inch stainless steel tubing to keep it straight. The testing temperature was set to be $550^{\circ} \mathrm{C}$ by an electric furnace. The testing time was 200 hours and the spectrum data was collected in every 5 hours. Figure 36 shows the transmission spectra of the LPFG at the 0 hour and 200 hour, respectively. Figure 37 plots the resonance wavelength changes as the time. We were happy to see that the grating kept its strength of resonance after the 200 hours test. However, its resonance wavelength drift quite bit from $1578.8 \mathrm{~nm}$ to $1553.5 \mathrm{~nm}$. We also noticed that the resonance wavelength changed much faster at the beginning than that at the end of the test. We believe this is due to the annealing effects which release the built in stress resulted from the laser irradiations. We are currently investigating the reasons that caused the resonance wavelength drifting towards the short 
wavelength, which might be the stress relief has different amount in the core vs. that in the cladding.

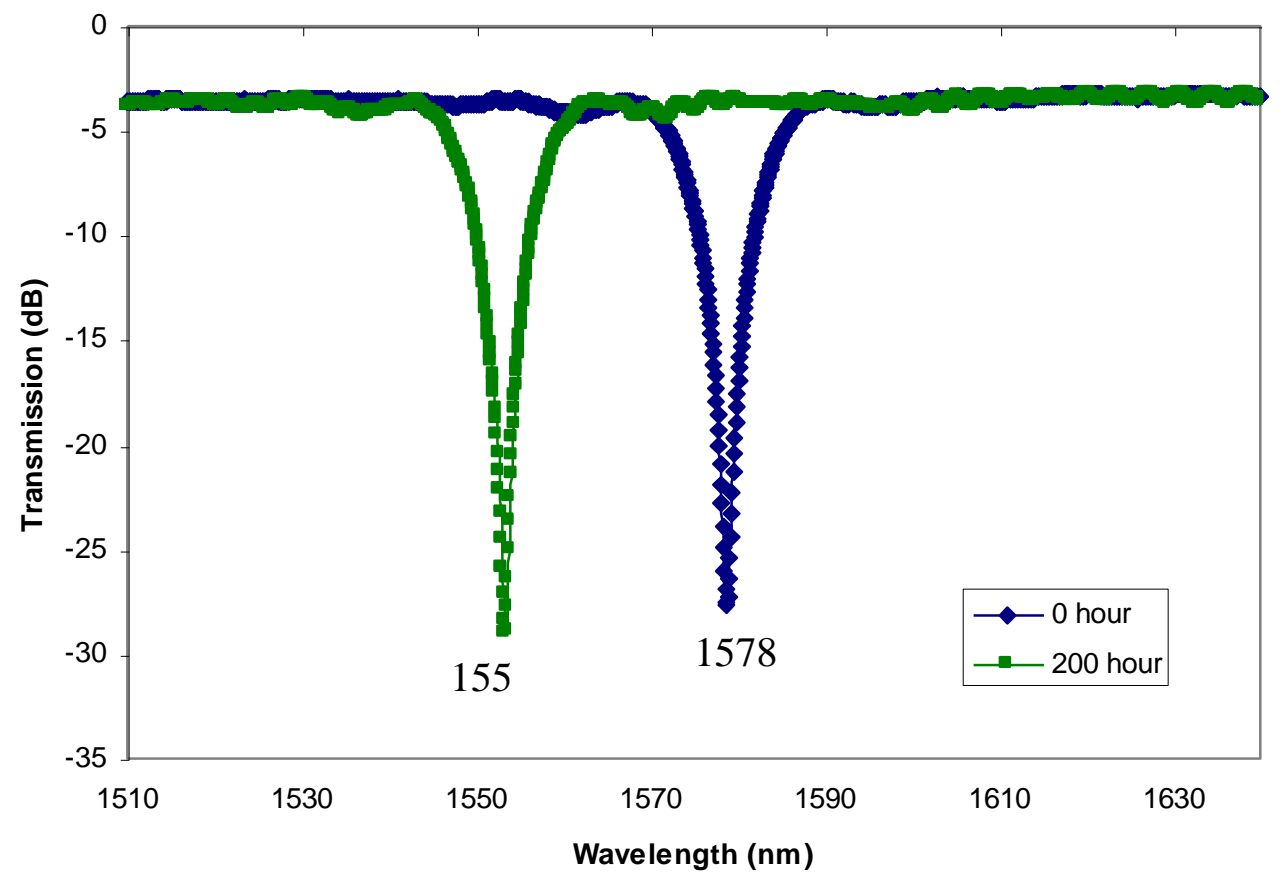

Figure $36 \mathrm{LPFG}$ spectrum before and after 200 hours aging at $550^{\circ} \mathrm{C}$ 


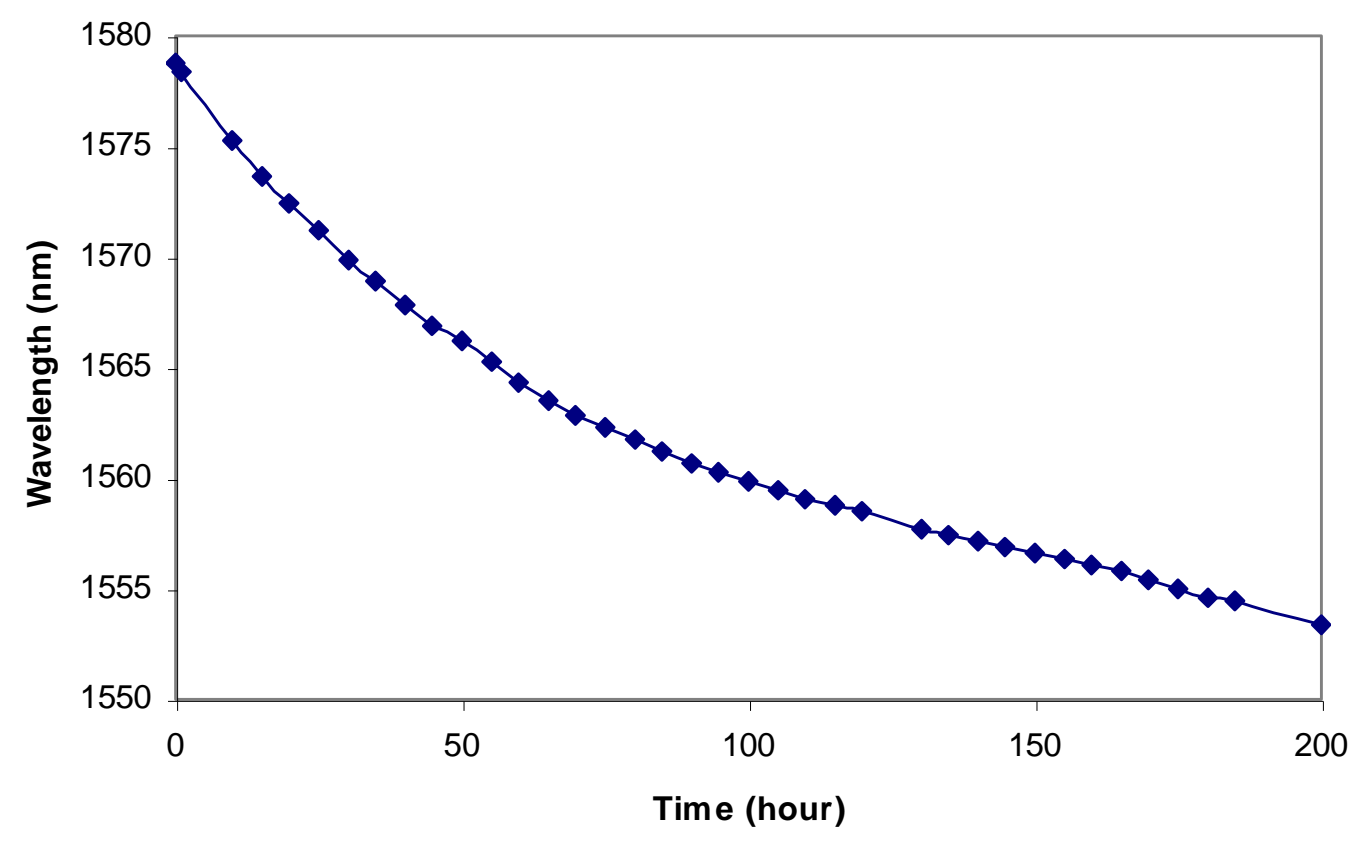

Figure $37 \mathrm{LPFG}$ resonance wavelength change as a function of time at $550^{\circ} \mathrm{C}$

We also investigated the method of improving the thermal stability of the LPFGs. Knowing that the lager thermal drift is most likely caused by the stress relief, we could first annealing the grating at a higher temperature, for example at $800^{\circ} \mathrm{C}$. We conducted the experiment by first annealing the grating at $800^{\circ} \mathrm{C}$ for one hour, then repeated the stability test at $550^{\circ} \mathrm{C}$ for 200 hours. The grating kept its resonance spectrum after the annealing process and showed a much smaller drift. Figure $\mathbf{3 8}$ shows the grating transmission spectrum at 0 hour and 200 hour at $550^{\circ} \mathrm{C}$. Figure 39 plots the LPFG resonance wavelength as a function of time. We can see that the resonance wavelength drifted from $1593.9 \mathrm{~nm}$ to $1591.0 \mathrm{~nm}$ for 200 hours at $550^{\circ} \mathrm{C}$, which is much smaller than that without annealing. 


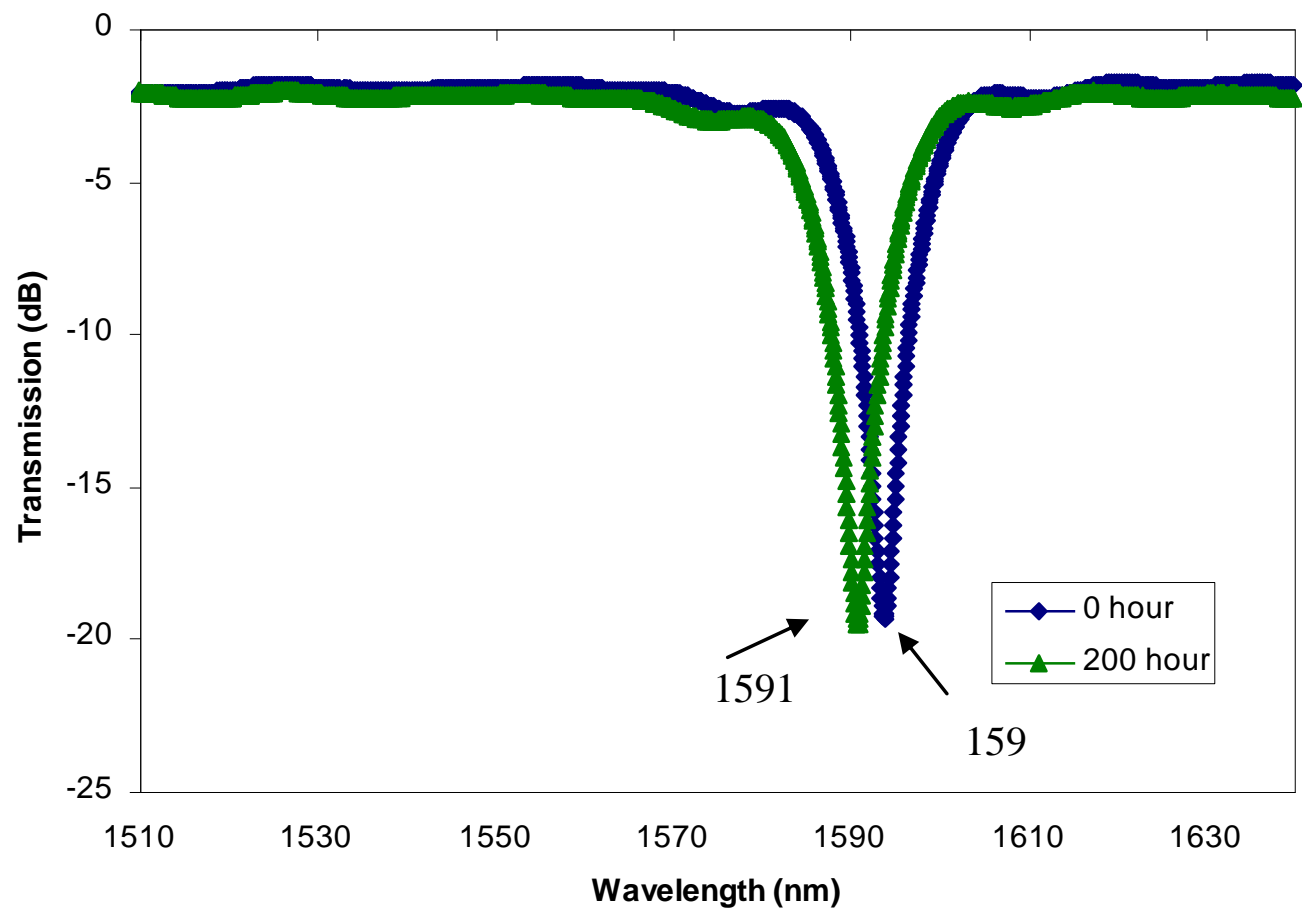

Figure $38 \mathrm{LPFG}$ spectrum at 0 hour and 200 hour at $550^{\circ} \mathrm{C}$ after annealing at $800^{\circ} \mathrm{C}$ for 1 hour

We believe there is still some room for improvement, which is currently under investigation both theoretically and experimentally. 


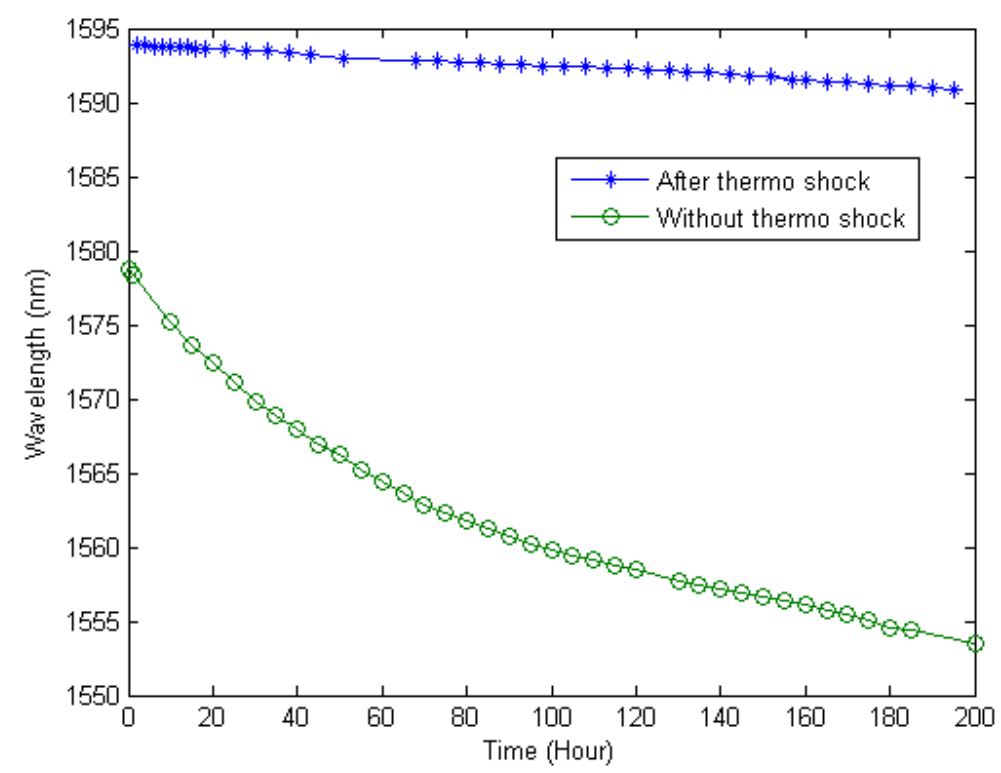

Figure $39 \mathrm{LPFG}$ resonance wavelength drift at $550^{\circ} \mathrm{C}$ after annealing at $800^{\circ} \mathrm{C}$ for 1 hour

Other experiments with longer duration time were conducted at MST to test the long term stability of the fabricated LPFGs. The first experiment was performing using as a LPFG fabricated using Corning SMF-28 singlemode fiber. The LPFG was hosted inside a 1/8-inch stainless steel tubing to keep it straight. The testing temperature was set to be $550^{\circ} \mathrm{C}$ by an electric furnace. The testing time was 60 days and the spectrum was collected in every day. Figure 40 plots the resonance wavelength changes as the time. Figure 41 shows the peak strength of the grating within the test period. We were happy to see that the grating survived the entire test duration of 60 (1440 hours) days even with two times of unexpected power outrage. This means that the sensor still operates after subjected to the high temperature harsh environment for two months. However, its resonance wavelength drift quite bit from. We also noticed that the resonance wavelength changed much faster at the beginning than that at the end of the test. We believe this is due to the annealing effects which release the built in stress resulted from the laser irradiations. 


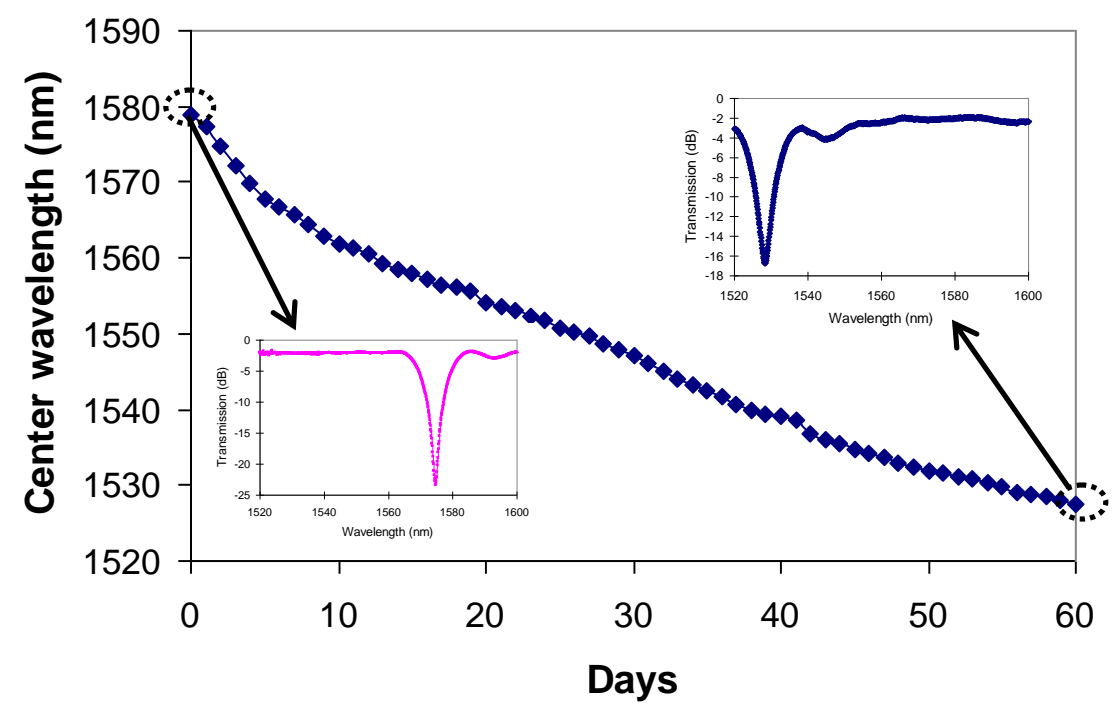

Figure 40 Drift in resonance wavelength of LPFG within 60 days in $550^{\circ} \mathrm{C}$. Inserts: transmission spectra of LPFG at the beginning and ending of the test, respectively.

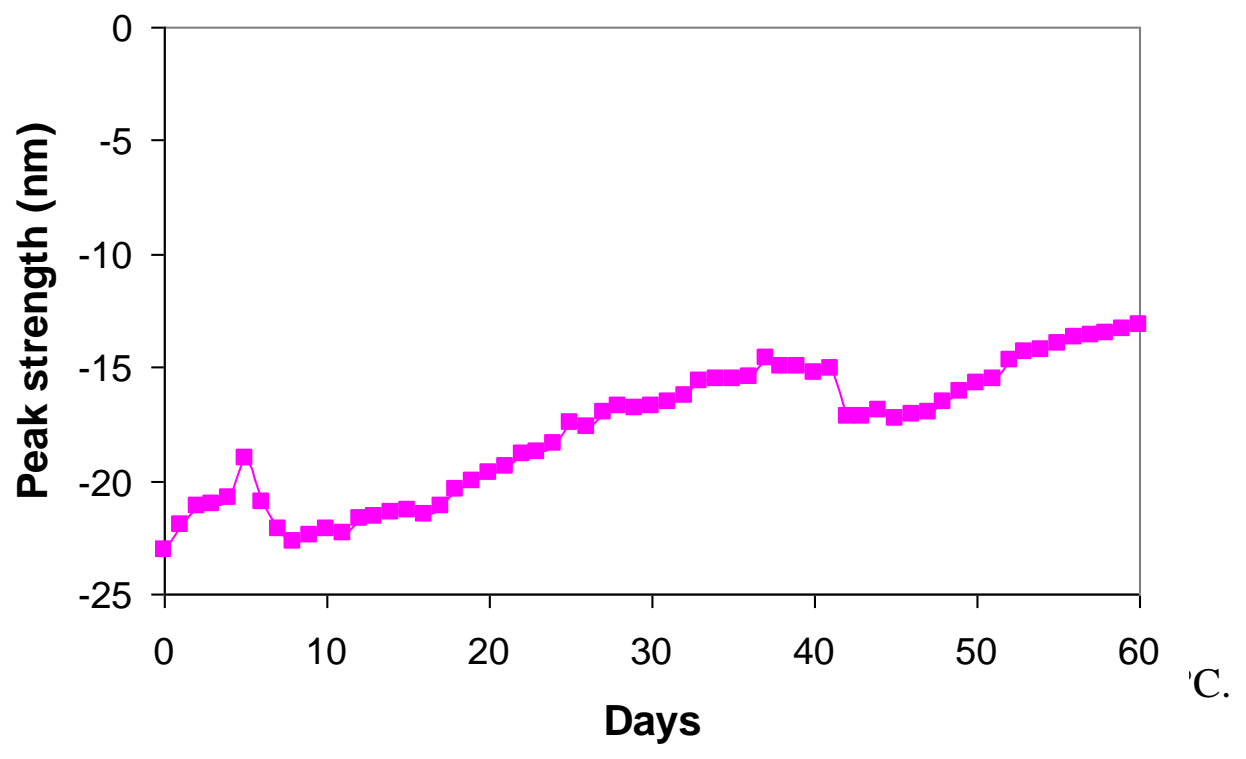

Figure 41 Drift in peak strength of LPFG within 60 days in $550^{\circ} \mathrm{C}$. 


\subsection{Fiber Ring Laser Interrogated Zeolite Coated SMS Structure for Trace Chemical}

\section{Detection}

Low-cost sensors capable of fast and sensitive detection of trace chemicals have attracted increasing interests in recent years due to their broad applications in environmental management, biological analysis, industry process control, energy production and homeland security. Among the many potential techniques, fiber optic chemical sensors have shown the advantages of small size, fast response, high sensitivity and immunity to electromagnetic interference (EMI). One of the directions in fiber optic chemical sensor development is to integrate chemically functional materials with various fiber optic devices. The chemical molecules interact with the functional material to cause its property changes (e.g., refractive index) that can be further amplified by the fiber device for sensitive detection.

Various porous materials have been explored for fiber optic chemical sensor development. The pore size of these materials ranges from a few angstroms to a few hundreds nanometers. Among the various porous materials, zeolites, a series of crystalline aluminosilicate materials with uniform subnanometer- or nanometer-scale pores, have shown the unique combination of chemical and optical properties for optical chemical sensing. Molecules from the surrounding environment can be efficient adsorbed and concentrated into the zeolitic pores for sensitive detection. Zeolite thin films have been synthesized onto optical fiber endfaces and the surface of long period fiber gratings (LPFGs) to develop fiber chemical sensors. Upon loading and unloading the guest molecules in the zeolitic pores, the coated zeolite thin film changes the refractive index that is measured by the fiber device. By modifying the material chemistry and surface properties of zeolite, the selectivity and sensitivity of the zeolite-fiber sensors can further be enhanced. 
In this section, we demonstrated a singlemode-multimode-singlemode (SMS) fiber structure coated with MFI zeolite thin film and interrogated by a fiber ring laser for highly sensitive chemical vapor detection. The SMS structure is very easy to fabricate, simply by fusion-splicing a section of multimode fiber between two singlemode fibers. Based on multimode interference and underlying self-imaging effect, the SMS structure functions as an optical band filter that has been widely explored for optical communication and sensing applications. The multimode section of the SMS structure can be made claddingless. As such, the light propagating inside can directly interact with the surrounding medium to monitor the changes in the refractive index of the environment.

One of the advantages of using SMS structure to fabricate the proposed zeolite-fiber chemical sensor is the fusion-spliced solid SMS structure can survive the high temperature, high pressure conditions that are required during the hydrothermal based zeolite synthesis/coating process. However, the SMS structure produces a broad optical band in spectrum, resulting in a small Q-factor and thus low resolution in the measurement of spectral shift. In this paper, we propose a fiber ring laser configuration to interrogate the zeolite-coated SMS sensor for improved detection accuracy.

The schematic configuration of proposed zeolite-coated SMS fiber sensor is shown in Figure 42(a). The SMS structure was made by fusion-splicing an input singlemode fiber (SMF), a section of multimode fiber (MMF) with the length of $5.25 \mathrm{~cm}$, and an output SMF together. To make the structure sensitive to ambient refractive index, the sandwiched MMF was made claddingless by dipping a section of MMF $(105 \mu \mathrm{m}$ in core diameter and $125 \mu \mathrm{m}$ in cladding diameter, Fiberguide Industries, Inc.) into a hydrofluoric acid solution (55\% concentration) for about two minutes to remove the fiber cladding. The diameter of the etched MMF was about 
$100 \mu \mathrm{m}$. After etching, the MMF was coated with a thin layer of MFI zeolite film on the surface according to synthesis process in the section 2. As shown in scanning electron microscope (SEM) images in Figure 42(b) and (c), the MMF was covered with a dense polycrystalline zeolite thin film. The thickness of the coated zeolite layer was about $3 \mu \mathrm{m}$, measured from the cross-sectional SEM image of the coated fiber.

The SMS structure is optical band pass filter as a result of the multimode excitation and interference inside the MMF, as well as the mode-filtering effect at the receiving single mode fiber. The peak wavelength of the band pass filter can be expressed as:

$$
\lambda_{0}=p\left(\frac{n_{M M F} D_{M M F}^{2}}{L}\right), p=0,1,2 \ldots
$$

where $\mathrm{n}_{\mathrm{MMF}}$ and $\mathrm{D}_{\mathrm{MMF}}$ are the effective refractive index and diameter of the fundamental mode of the MMF, $\mathrm{p}$ is an integer corresponding to the self-image number, and $\mathrm{L}$ is the length of the MMF.

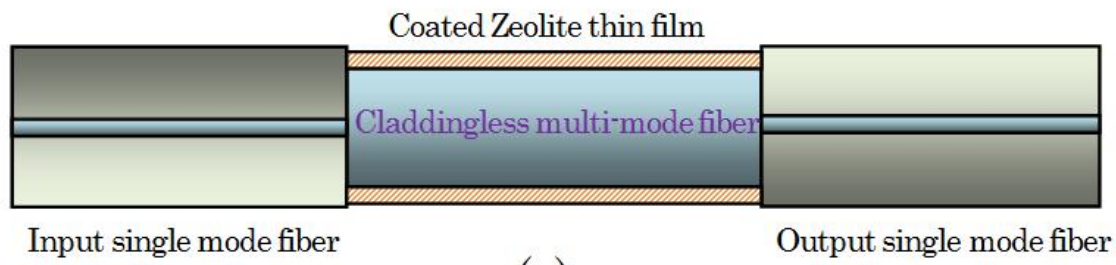

(a)

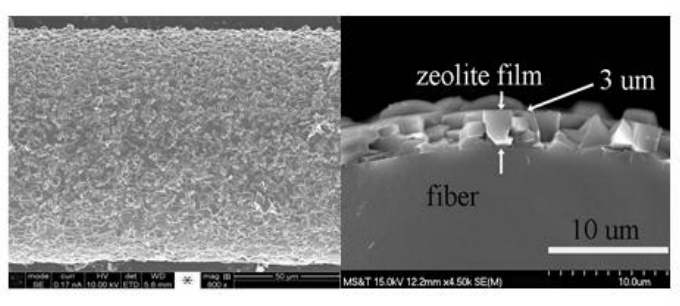

(b) (c)

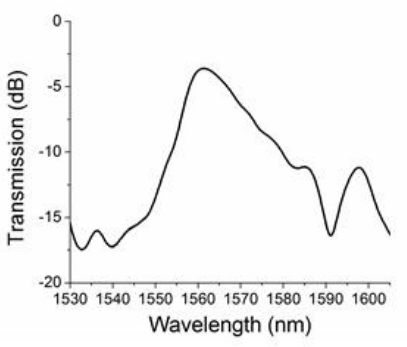

(d)

Figure 42 Zeolite coated SMS fiber structure. (a) Device schematic, (b) and (c) SEM images of zeolite-coated optical fiber, (d) Transmission spectrum of the zeolite-coated SMS structure. 
The coated zeolite thin film acts as the fiber cladding which can change its refractive index by adsorbing from and de-adsorbing chemical molecules to the surrounding environment. When molecules load into the zeolite cavity, the effective refractive index and the effective diameter of the fundamental mode of the MMF will be increased due to Goos-Hanchen shift and evanescent

filed penetration increase, respectively. The higher the chemical concentration is, the larger the adsorption and thus the larger the changes become. According to Eq. (1), when $\mathrm{n}_{\mathrm{MMF}}$ and $D_{M M F}$ change, the peak wavelength of the band pass filter shifts correspondingly. The amount of wavelength shift can thus be measured to find the chemical concentration in the local environment.

However, the SMS based band pass filter has a broad spectrum (or low Q-factor) as shown in Figure 42(d). The broad spectral width results in a poor resolution for measurement of spectral shift. As such, the as-constructed zeolite-SMS chemical sensor is expected to have a poor detection limit. To improve the detection limit, a fiber ring laser is constructed to interrogate the zeolite coated SMS as schematically illustrated in Figure 43.

A section of erbium-doped fiber (EDF, Lucent HP980) with a length of 5m was used as the gain medium. A C-band optical fiber isolator was inserted into loop to keep unidirectional light propagation. The laser was pumped using a $974 \mathrm{~nm}$ wavelength laser diode with a power of 50mW through a 980/1550 nm wavelength-division-multiplexing (WDM) fiber coupler. A 2/98 fiber coupler was used to monitor the peak wavelength of the laser. At the $2 \%$ output port of the coupler was connected to an optical spectrum analyzer (OSA, AQ 6370), where the laser spectrum was recorded by the computer continually. 


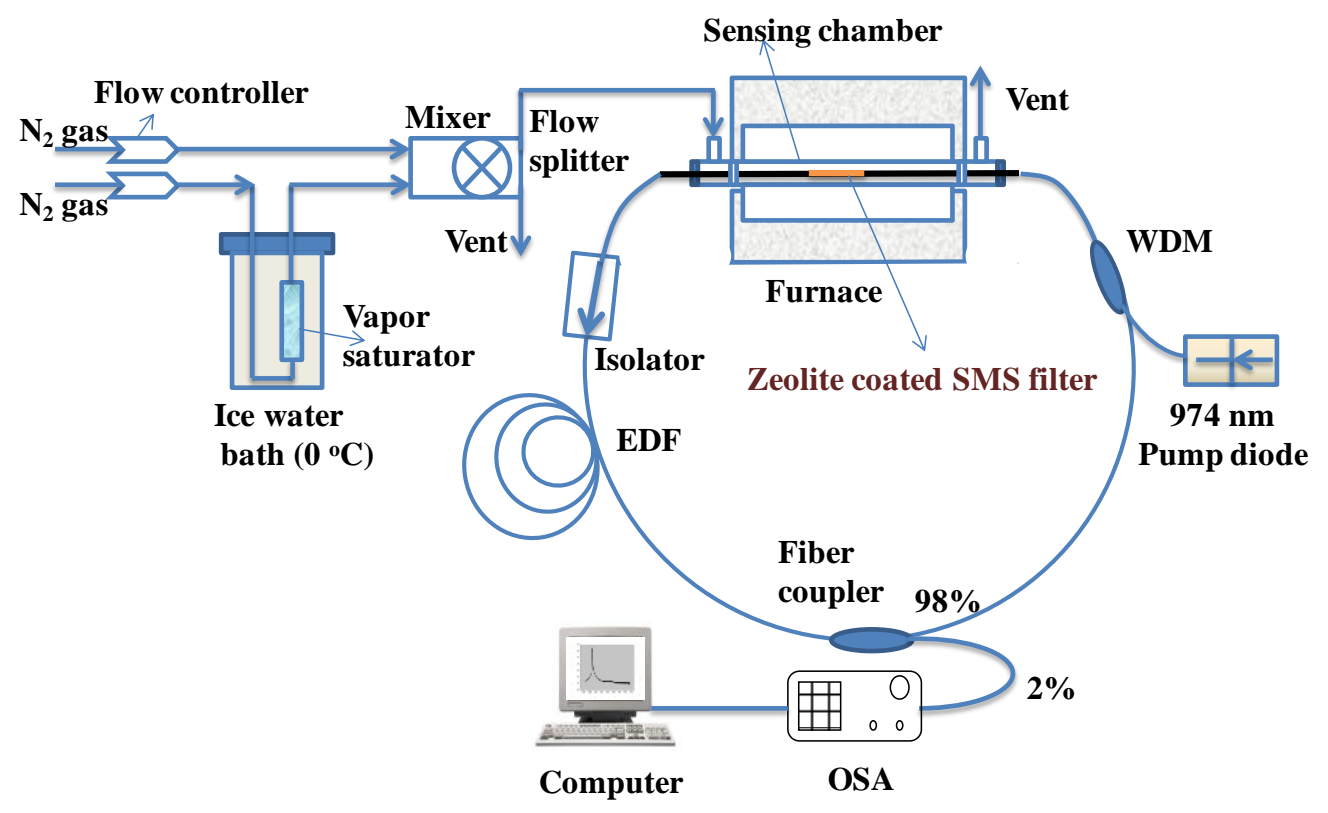

Figure 43 Experimental setup for sensor evaluation and schematic of fiber ring laser

The experiment setup for testing the zeolite-coated SMS structure in response to vapor is also schematically shown in Figure 43. The zeolite coated SMS structure was placed in a test chamber made of 1/4-in I.D. stainless-steel tube, which was placed horizontally in a tubular furnace. The two ends of the stainless stell tube were connected to the sample gas/vapor supply system and ventilation, resprectively. $\mathrm{N}_{2}$ gas from a cynlinder was used as the carrying gas. One path was connected to a liquid bubbler placed in a $0{ }^{\circ} \mathrm{C}$ ice-water bath. In this paper, ethanol (sigma-aldrich, $\geq 99.8 \%$ ) vapor was used to evaluate the sensor. The other path was directly connected to the vapor path through a mixer to further dilute the concentration. Flow controlers were used to regulate the gas flow rate so that the vapor concentration could be precisely varied. The concentration of the ethanol vapor was calculated based on the vapor pressure at $0{ }^{\circ} \mathrm{C}$ and diluting gas flow rate. All the tests were conducted at room temperature and local atmospheric pressure. 
After the thermal activation of zeolite at $500^{\circ} \mathrm{C}$ in the furnace shown in Figure 43, the zeolite-coated SMS fiber ring laser sensor was tested for detection of low concentraion of ethanol vapor in $\mathrm{N}_{2}$. Figure 44 shows the transmission spectra of the zeolite-coated SMS structure at various ethanol vapor concentrations. Compared with the non-lasing spectrum shown in Figure 42(d), the lasing spectrum had a much narrower bandwidth and much higher signal-tonoise ratio. The side mode suppression ratio was estimated to be $55 \mathrm{~dB}$ and the $3 \mathrm{~dB}$ bandwidth was about $0.2 \mathrm{~nm}$ measured by the OSA. With the high signal-to-noise ratio and narrow laser bandwidth, this fiber ring laser can work as a chemical vapor sensor with a low detection limit.

As the vapor concentraion increased, the fiber laser peak would shifted to longer wavelength. This observation is in good agreement with that predicted by Eq. (1). The wavelength shift is resulted from the increase of the effective refractive index of zeolite film induced by the increasing sorption of organic molecules into the zeolite cavity. In addition, the output laser peak powers were almost at the same value, indicating that there was no obvious transmission loss during vapor adsorbtion in this experiment.

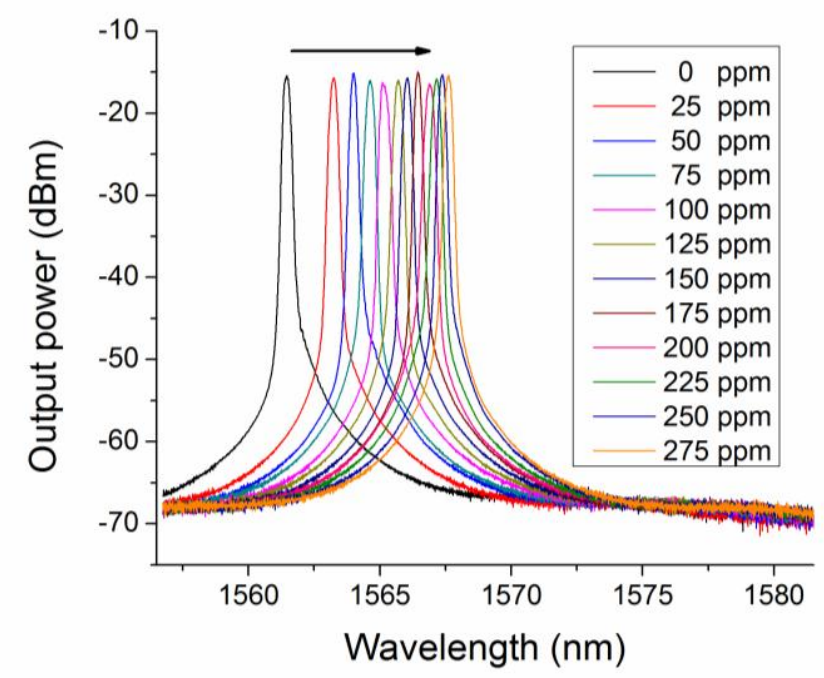

Figure 44 Lasing spectra of the zeolite-coated SMS structure at various ethanol concentrations. 


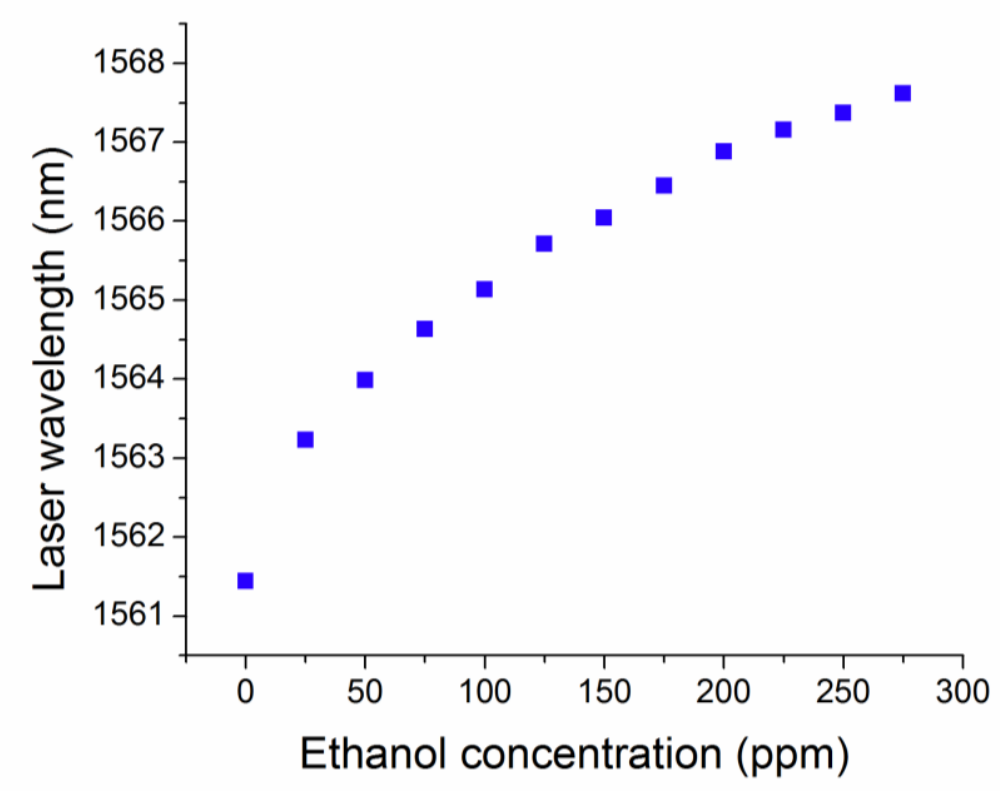

Figure 45 Center wavelength of the zeolite-coated SMS fiber ring laser as a function of ethanol vapor concentration.

Figure 45 plots the lasing wavelength as a function of the ethanol vapor concentrations, which varied from $0 \mathrm{ppm}$ to $275 \mathrm{ppm}$. The relation between the lasing wavelength and the concentration shown in Figure 45 exhibits a non-linear behavior. This is mainly because the adsorption of molecules into the zeolite pores is not linear. The adsorption approaches a saturation state when the concentration keeps increasing. In general, the sensor has higher sensitivity at a lower concentration level. Nevertheless, the sensor device responded to the vapor concentration monotonically, with a clear correlativity for quantitative measurement after calibration.

To test the stability of the device, the peak wavelength of the laser was monitored for three hours of operation at room temperature. The standard deviation of the peak wavelength was found to be $\pm 0.022 \mathrm{~nm}$, indicating a good stability of the system. 
In summary, zeolite thin films have been successfully synthesized on the surface of a claddingless optical fiber in a SMS structure to construct a chemical vapor sensor. The zeolitecoated SMS structure was used as a band pass filter and inserted into an Erbium fiber loop to generate a laser line with narrow spectrum width and high signal-to-noise ratio. The nanoporous zeolite adsorbs chemical molecules from the surrounding environment to increase its effective refractive index of the coated zeolites, producing a wavelength shift of the SMS filter, and correspondingly the change in lasing wavelength. The sensor has been demonstrated for detection of ethanol vapors at room temperature. Combining the strong molecular adsorption characteristics of zeolite and the high signal-to-noise ratio of the laser signal, the device can be used as a highly sensitive in situ sensor with a low vapor detection limit. This novel fiber based sensor platform can also be found useful in the study of molecular adsorption dynamics in zeolite structures which may benefit the design and characterization of new zeolitic materials for applications in chemical separation and process control. 


\section{Sensor Material Development}

The research in the area of material development was focused on four areas including 1) sensor material synthesis, identification, and characterization, 2) nanomaterial synthesis, processing and fiber coating, 3) optical fiber devices design, fabrication, and characterization, and 4) Laboratory and field testing the sensor devices. Initial phases of the research were focused on processing and characterizing nano-crystalline YSZ, SDC, and SDC/YSZ hetero-phase thin films. These materials were established to be suitable for detection of $\mathrm{H}_{2}$, and $\mathrm{CO}$ based on oxygen partial pressure changes. Dense nano-crystalline SDC/YSZ hetero-phase thin films were successfully synthesized on $\mathrm{MgO}$, sapphire, and silicon wafers from SDC-nanoparticleincorporated polymeric precursors. At reduced temperatures, the nanostructured YSZ/SDC hetero-phase film exhibited total electrical conductivity significantly higher than that of the phase-pure YSZ and SDC nano-crystalline thin films with comparable grain sizes. The refractive indices of the doped-ceramic nano films indicate that the materials are appropriate for use as optical sensor materials.

The sensing materials for $\mathrm{H}_{2}$, and $\mathrm{CO}_{2}$ gases at high temperatures were identified by the ASU team. The nanomaterial processing and coating methods were successfully demonstrated on flat surface substrates as well as on optical fibers by Dong's group at NMT.

In the later phases of the project, research was focused on 1) processing the identified sensing material into nano-crystalline thin films, 2) integrating these nano films with the fiber devices to construct high temperature gas sensors, and 3) testing and evaluating the sensor performance. The research on sensor material development specifically was focused on 1) coating $\mathrm{Pd}$ (Palladium) thin film on fiber by sputtering deposition and electroless plating, 2) grain size 
modification of the coated $\mathrm{Pd}$ particles by thermal treatment, and 3) refractive index measurement of Pd thin film deposited on a dense alumina disk.

Several perovskite type ceramics exhibit proton-conductivity in hydrogen containing atmosphere at high temperatures. Thus, the proton-conducting perovskite type ceramics offer potential as hydrogen sensing material in the optical, electrochemical and resistor type sensor devices. For optical sensor application, the proton-conductivity change of perovskite type ceramics in response to a change in hydrogen partial pressure should be related to their optical properties. 


\subsection{Synthesis and Characterization of proton-conducting Ceramics}

The materials studied included $\mathrm{SrCeTm}_{0.05} \mathrm{O}_{3-\delta} \quad(\mathbf{S C T m}), \quad B a C e N d_{0.1} \mathrm{O}_{3-\delta} \quad(\boldsymbol{B C N d})$, $\mathrm{SrCeTb}_{0.05} \mathrm{O}_{3-\delta}(\mathbf{S C T b}), \mathrm{SrCeTb}_{0.1} \mathrm{O}_{3-\delta}(\mathbf{S C T b})$ and $\mathrm{BaCeTb}_{0.05} \mathrm{O}_{3-\delta}(\boldsymbol{B C T b})$. These materials were selected to study the effects of the dopant on the electrical properties of strontium cerate and barium cerates - the two best studied proton-conducting ceramics. Citrate method was identified to be effective for synthesis of these ceramics. These materials with relative density larger than 95\% were obtained by the citrate method. XRD analysis shows that the materials synthesized have desired perovskite phase structure.

A high temperature electrical conductivity apparatus was set up and used to measure the electrical conductivity of these materials in different atmospheres at high temperatures (by the four point method). The conductivity data of these materials under He or air atmospheres in $500-900^{\circ} \mathrm{C}$ are shown in Figure 46. Due to safety requirement, a special hydrogen cylinder storage cabinets and hydrogen transport pipeline were built at the ASU team's lab. The system was used for $\mathrm{H}_{2}$ experiments.

As shown in Figure 46, a change in the measuring environment from He to air has almost no effect on the conductivity of $\mathrm{SrCeTb}_{0.05} \mathrm{O}_{3-\delta,} \mathrm{SrCeTb}_{0.1} \mathrm{O}_{3-\delta}$, and $\mathrm{BaCeTb}_{0.05} \mathrm{O}_{3-\delta}$ as compared to $\mathrm{SrCeTm}_{0.05} \mathrm{O}_{3-\delta}$ and $\mathrm{BaCeNd}_{0.1} \mathrm{O}_{3-\delta}$. This indicates that when $\mathrm{Tb}$ is used as the dopant for the strontium or barium cerate, electronic conductivity is quite low. This was consistent with the results reported by X. Qi and Y.S. Lin [solid State Ionics 120 (1999) 85-93]. Tb doped strontium cerate also showed good proton-conductivity. Conductivity was measured in $\mathrm{H}_{2}$ to investigate the effect of $\mathrm{H}_{2}$ partial pressure on the conductivity of these materials.

Pd based hydrogen optical or resistor sensors have been studied extensively in the last decade. Once exposed to $\mathrm{H}_{2}$, formation of $\mathrm{PdH}$ will change both the electrical conductivity and 
optical property of the metal. A literature search shows that most studies were focused on the application of $\mathrm{Pd}$ based $\mathrm{H}_{2}$ sensors for the $\mathrm{H}_{2}$ leak detection $\left(\mathrm{H}_{2}\right.$ concentration lower than the $4 \%$ ) at relative low temperatures. The studies of its application at high temperatures for high $\mathrm{H}_{2}$ concentration (usually $10-50 \%$ in fossil fuel gases) were rarely reported.
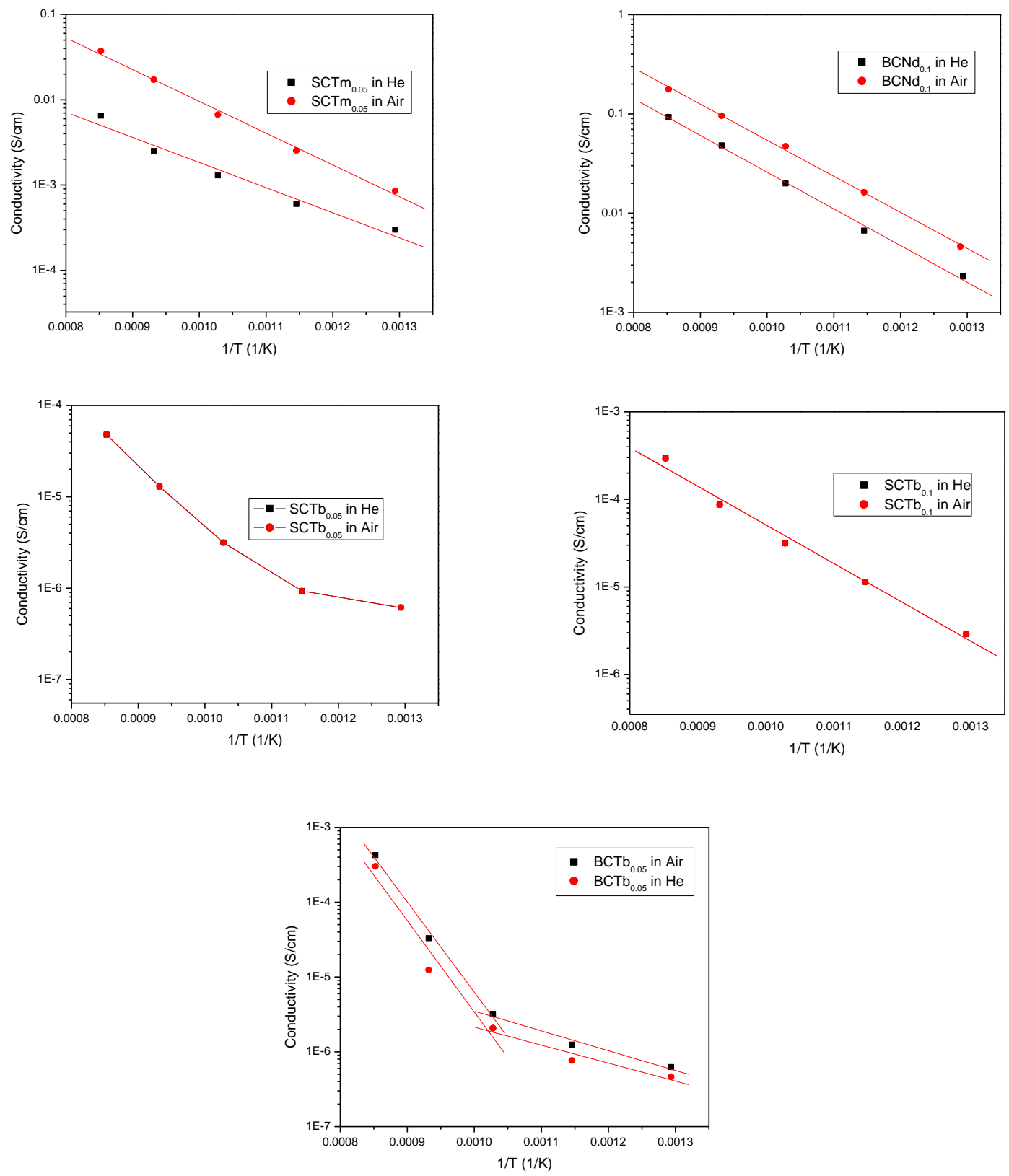

Figure 46. Electrical conductivity of synthesized perovskite type ceramics under $\mathrm{He}$ and air. 
Electroless plating and sputter deposition methods were selected for synthesis of Pd and Pdalloy of different nanostructure. Once nanostructured Pd and Pd alloy were synthesized, the ASU team studied the electrical conductivity, lattice parameters, and hydrogen solubility and mobility of the Pd and Pd-alloy at various hydrogen partial pressures. The study also included the effects of nanostructure (e.g., grain size) on these properties of Pd and Pd alloy. These properties are directly related to the performance of the optical sensor using the metals. 


\subsection{Synthesis of nano-crystalline SDC/YSZ heterophase thin films on flat substrates}

The SDC/YSZ heterophase thin film electrolytes were synthesized on MgO and Si substrates by spin coating and thermal treatment of SDC-nanoparticle-incorporated polymeric precursors. The SDC nano-powders were obtained commercially from NexTech, Ohio. The as-received nano-powders had a composition of $\left(\mathrm{Ce}_{0.85} \mathrm{Sm}_{0.15}\right) \mathrm{O}_{1.925}$ and grain size of $5-10 \mathrm{~nm}$. The SDC powders underwent pretreatments by firing in air for $10 \mathrm{~h}$ at different temperatures. The average SDC crystallite sizes increased to 25,35 , and $45 \mathrm{~nm}$ after firing at 800,1000 , and $1200^{\circ} \mathrm{C}$, respectively.

The SDC-nanoparticle-incorporated polymeric precursor was synthesized by the following procedure: (1) The YSZ polymeric precursor, $\left(\mathrm{Zr}^{4+}, \mathrm{Y}^{3+}\right)$-chelated poly ethyl glycol, was synthesized by polymerization of an aqueous solution containing ethylene glycol, zirconyl chloride, yttrium nitrate, and glycine. The precursor had a $\mathrm{Y}: \mathrm{Zr}$ atomic ratio of 16:84. The detailed polymeric precursor synthesis procedure can be found in the literature. (2) A stable SDC colloidal suspension (1wt\% solid content) prepared by ultrasonic dispersion under controlled $\mathrm{pH}$ (= 3 4, adjusted by $0.1 \mathrm{~N} \mathrm{HNO}_{3}$ ) was added to the YSZ polymeric precursor drop-wise under stirring. (3) The mixture was then heated at $80^{\circ} \mathrm{C}$ under stirring for five hours to remove water and improve the viscosity for film coating. The SDC-nanoparticle-incorporated precursor was then cooled to room temperature under stirring and used to deposit thin films on <100> magnesium oxide $(\mathrm{MgO}, 18 \mathrm{~mm} \times 18 \mathrm{~mm} \times 0.5 \mathrm{~mm})$ and $<001>$ silicon wafers.

The typical spin-coating process included a 5 -second spinning at $600 \mathrm{rpm}$ followed by a 20 second spinning at $2500 \mathrm{rpm}$ using a two-stage spin-coater (KW-4A, CheMat, CA). After coating, the film was dried at $80^{\circ} \mathrm{C}$ and fired at $300^{\circ} \mathrm{C}$ for one minute on a hot plate. The film 
was then fired at $650^{\circ} \mathrm{C}$ in air for four hours. After firing, the films were further annealed at $750^{\circ} \mathrm{C}$ for five hours. The purpose of the $750^{\circ} \mathrm{C}$-annealing step was to prevent grain growth during the conductivity measurements in a temperature range of $400-750^{\circ} \mathrm{C}$. The film coating process was repeated for three more times that resulted in a final oxide film thickness of $\sim 140$ nm. Thicker films were prepared by additional coatings under identical conditions to obtain strong XRD peak intensities for examining the crystal phase and grain sizes in the films.

The phase-pure YSZ and SDC nano crystalline thin films were synthesized using similar procedures as described in the literatures. The atomic ratios of $\mathrm{Y}: \mathrm{Zr}$ and $\mathrm{Sm}: \mathrm{Ce}$ in their respective polymeric precursors were 16:84 and 15:85, respectively. Thus the chemical composition of the phase-pure YSZ and SDC films were $\left(\mathrm{Zr}_{0.84} \mathrm{Y}_{0.16}\right) \mathrm{O}_{1.92}$ and $\left(\mathrm{Ce}_{0.85} \mathrm{Sm}_{0.15}\right) \mathrm{O}_{1.925}$, respectively. The YSZ and SDC nanocrystalline films were obtained by the same thermal treatments as used in the preparation of the heterophase films, namely firing at $650^{\circ} \mathrm{C}$ for four hours followed by annealing at $750^{\circ} \mathrm{C}$ for five hours. Both the pure YSZ and SDC films had a film thickness of $\sim 100 \mathrm{~nm}$ using a total of four coating steps. After annealing at $750^{\circ} \mathrm{C}$, the grain sizes of the $\mathrm{YSZ}$ and SDC thin films were both $\sim 15 \mathrm{~nm}$. 


\subsection{Nanomaterial processing and coating}

Nanocrystalline ceramic matrices with desired physical, chemical, mechanical and optical properties have been developed for gas sensing. The methods of integrating these material matrices with fiber sensor platforms were also developed.

Nanocrystalline thin films (thickness $100 \sim 200 \mathrm{~nm}$ ) of the identified doped-ceramics and their heterophases have been successfully synthesized on the cylindrical surface of the LPFG by the polymeric precursor method. The microscopic images (SEM and TEM) of the coatings indicated excellent film integrity, uniformity, continuity, and film-fiber interface. The coated thin films successfully survived high temperatures up to $700^{\circ} \mathrm{C}$ without observable sign of performance degradations. The nano crystalline nature and the hetero-phase structure of the film were studied and confirmed by total electric conductivity measurements at high temperatures. The key to success of coating the nano films on the fibers is to control the precursor viscosity appropriate for film formation. For both YSZ and SDC coating, a polymerization time of 2 to 15 hours and reaction temperature of $80-90^{\circ} \mathrm{C}$ were found appropriate.

Micro-porous zeolite overcoat thin films (thickness $2 \sim 10 \mu \mathrm{m}$ ) were successfully synthesized on the end face and cylindrical surface of optical fibers using the in-situ hydrothermal crystallization method. SEM images of the coated fiber indicated excellent quality of the zeolite coat. The coated device survived high temperatures up to $650^{\circ} \mathrm{C}$ with no sign of physical degradation. The zeolite-LPGF structure was also tested to be thermally stable at $550^{\circ} \mathrm{C}$. By continuously monitoring the LPFG signal, the zeolite film synthesis and calcination processes were also investigated. 


\section{$\underline{\text { 2.3.1 Characterization and results }}$}

The SDC/YSZ heterophase films were first synthesized using SDC nano-crystals fired at different temperatures with a fixed SDC:YSZ molar ratio of 1:1, which gave a SDC volume fraction of 0.587 (SDC:YSZ volume ratio of 1:0.704), in the final oxide films. The pretreated SDC nano-powders and the as-received powders were used to prepare the SDC nanoparticleincorporated polymeric precursors. For all these powders including the untreated one, the SDC crystal structure was not changed in the precursor films after being dried at $80^{\circ} \mathrm{C}$ and further heated at $300^{\circ} \mathrm{C}$ according to the XRD tests.

The precursor films containing SDC particles without pretreatment and particles pretreated at $700^{\circ} \mathrm{C}$ were found to form YSZ-SDC solid solutions after firing at temperatures as low as $450^{\circ} \mathrm{C}$. Lines (d) and (e) in Figure 47 XRD patterns. Lines a, b and c are YSZ, SDC and YSZ/SDC solid solution pellets (Ref. 8), respectively. d and e are films synthesized with untreated SDC: d - fired at $300^{\circ} \mathrm{C}$ and e - fired at $450^{\circ} \mathrm{C}$ (solid solution). $\mathrm{f}, \mathrm{g}$ and $\mathrm{h}$ are films with SDC pretreated at $1200^{\circ} \mathrm{C}$ : f - dried at $80^{\circ} \mathrm{C}$, g - fired at $650^{\circ} \mathrm{C}$, and h - fired at $850{ }^{\circ} \mathrm{C}$.are $\mathrm{XRD}$ patterns of the precursor film containing untreated SDC powders after being fired at $300^{\circ} \mathrm{C}$ and the solid solution film after further firing at $450^{\circ} \mathrm{C}$. The precursor film containing SDC powders pretreated at 800 and $1200^{\circ} \mathrm{C}$ formed a SDC/YSZ hetero phase structure after firing at $650^{\circ} \mathrm{C}$. The hetero phase structure of these films was stable during further heat treatment at $850^{\circ} \mathrm{C}$. The lines (f), (g), and (h) are XRD patterns of the precursor film containing SDC powders pretreated at $1200^{\circ} \mathrm{C}$ after being fired at $300^{\circ} \mathrm{C}$ (for $1 \mathrm{~min}$ ), $650^{\circ} \mathrm{C}$ (for $4 \mathrm{hr}$ ), and $850^{\circ} \mathrm{C}$ (for $100 \mathrm{hr}$ ), respectively.

In the hetero phase thin films, the YSZ grain size was $\sim 15 \mathrm{~nm}$ after annealing at $750^{\circ} \mathrm{C}$ for five hours. The SDC grain sizes remained unchanged from their values after firing (pretreatment) 
at 800,1000 , and $1200^{\circ} \mathrm{C}$ which were 25,35 , and $45 \mathrm{~nm}$, respectively. The grain sizes in the films were estimated by XRD technique. In the following text, SDC800/YSZ, SDC1000/YSZ, and SDC1200/YSZ were used to denote the hetero phase films containing SDC particles pretreated at 800,1000 , and $1200^{\circ} \mathrm{C}$, respectively. No inter crystal pores or pinholes were found in the hetero phase films by surface examinations using Atomic Force Microscopy as shown in Figure 48The four-coat films had a thickness of $\sim 140 \mathrm{~nm}$ according to the observation by a Field Emission Scanning Electron Microscope (SEM, Hitachi S-5200 Nano-SEM) for the cross section of a Si-supported film as shown in Figure 49. A Si substrate was used here because it provided a better cross section sample for clearer SEM observations compared to the $\mathrm{MgO}$.

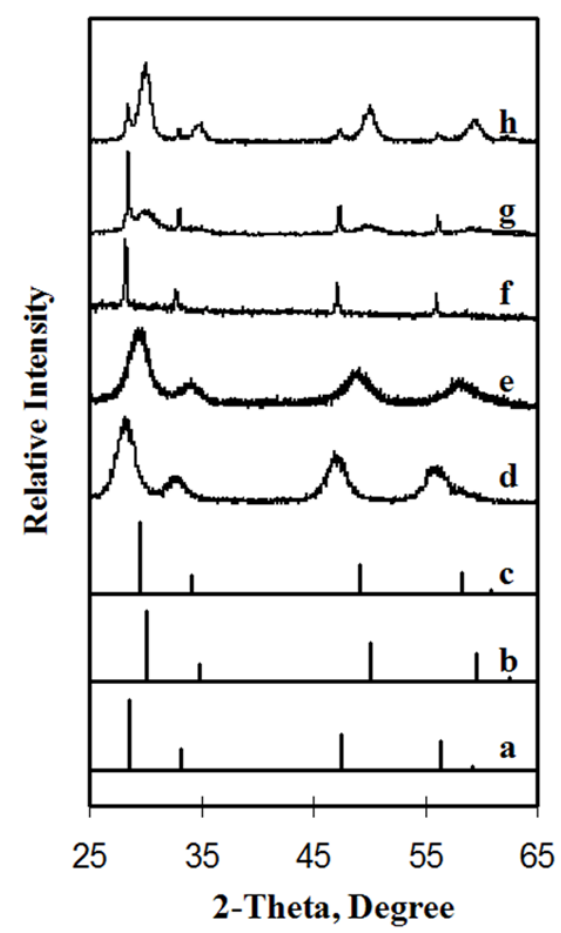

Figure 47 XRD patterns. Lines a, b and $\mathrm{c}$ are YSZ, SDC and YSZ/SDC solid solution pellets (Ref. 8), respectively. $\mathrm{d}$ and e are films synthesized with untreated SDC: $\mathrm{d}$ - fired at $300^{\circ} \mathrm{C}$ and e - fired at $450^{\circ} \mathrm{C}$ (solid solution). $\mathrm{f}, \mathrm{g}$ and $\mathrm{h}$ are films with SDC pretreated at $1200^{\circ} \mathrm{C}$ : $\mathrm{f}$ - dried at $80^{\circ} \mathrm{C}, \mathrm{g}$ - fired at $650^{\circ} \mathrm{C}$, and $\mathrm{h}$ - fired at $850^{\circ} \mathrm{C}$. 


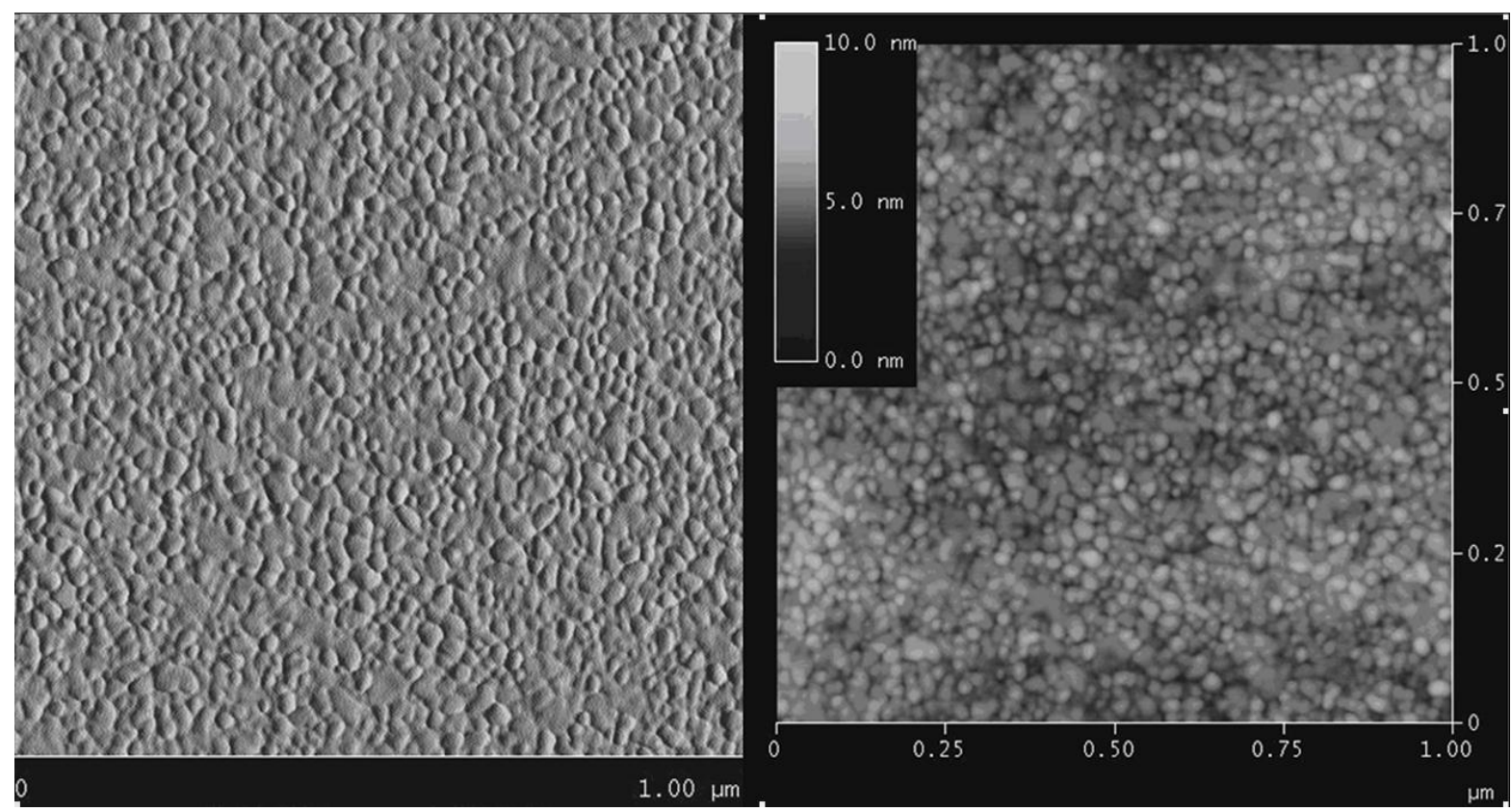

Figure 48 AFM image of the surface of the YSZ800/SDC heterophase film annealed at $750^{\circ} \mathrm{C}$ for 5 hours.

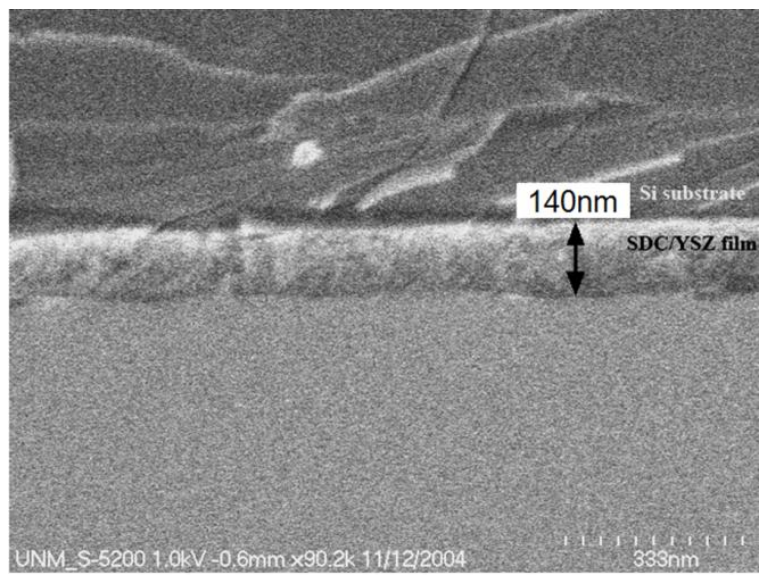

Figure 49. SEM image of the cross-section of the SDC800/YSZ heterophase film.

The grain size and density of the hetero-phase thin films were further confirmed by transmission electron microscopy (TEM) as shown in Figure 50(a) for a film containing SDC nano-crystals pretreated at $800^{\circ} \mathrm{C}$. Figure 50(b) is the image of Scanning TEM Z-contrast 
mapping (JEOL 2010 FEG TEM STEM - Oxford EDS, Gatan Imaging Filter ) for the cross section of a SDC800/YSZ hetero-phase film. The Z-contrast mapping shows that the SDC particles are uniformly dispersed in a matrix of YSZ nano-crystallites.
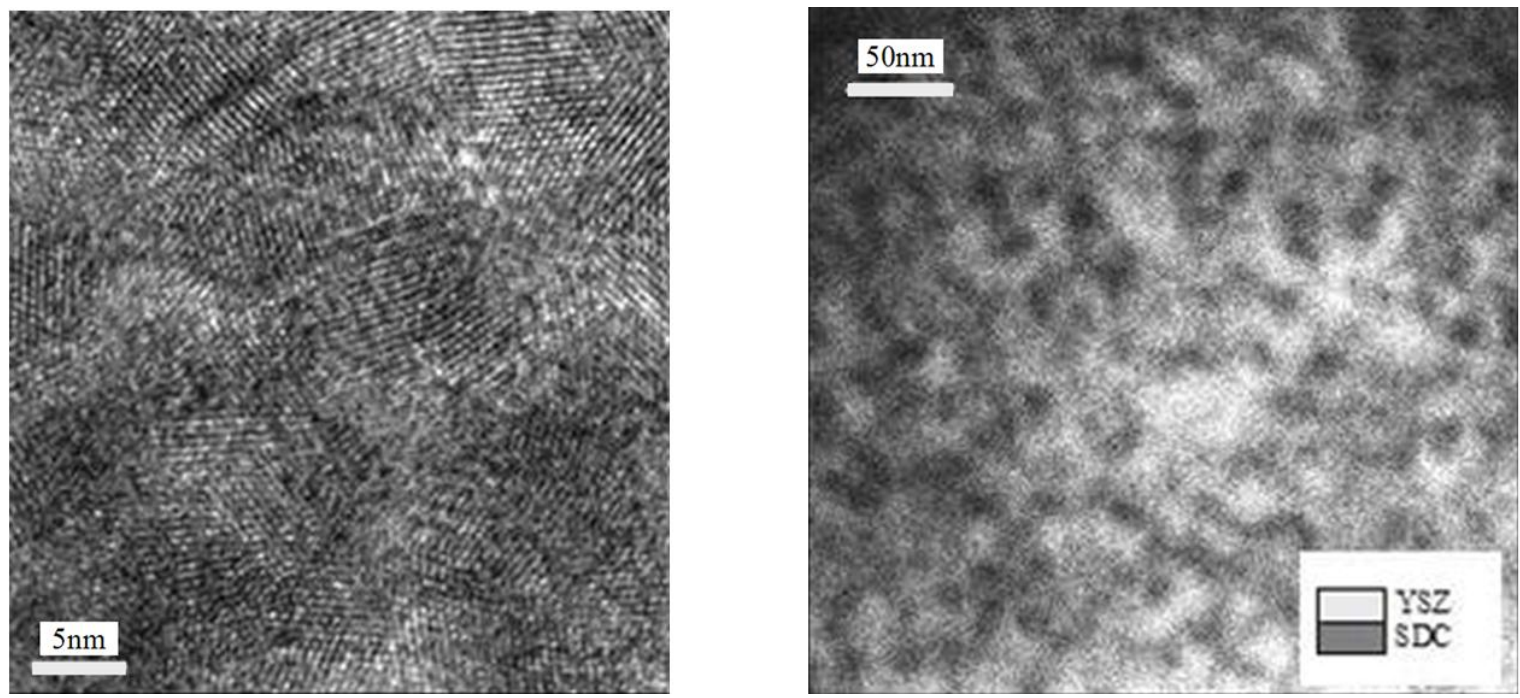

Figure 50. TEM observations of a SDC/YSZ hetero-phase film cross section. (a) TEM image; (b) STEM Z-contrast mapping (contrast inverted for better observations).

The total electrical conductivities of the MgO-supported nano-crystalline thin films were measured in air using the four-probe technique. The results are presented in Figure 51. The conductivity of the solid solution thin film was not measured because the potentiostat was unable to maintain a stable current under its maximum output voltage $(10-\mathrm{V})$ for the highly resistive thin film. The total conductivity of the nano-crystalline YSZ thin film obtained in this study agreed well with the literature value for a YSZ film synthesized under similar conditions with comparable grain size. With comparable grain sizes, the total electrical conductivity of the SDC800/YSZ hetero-phase thin film was 3 to 20 times that of the nano-crystalline SDC film in a temperature range of 450 to $700^{\circ} \mathrm{C}$ and was 2 to 12 times that of the $\mathrm{YSZ}$ nano-crystalline film in 
a temperature range of $500-700^{\circ} \mathrm{C}$. The extraordinarily high conductivity of the hetero-phase film at reduced temperatures may be attributed to the fast conduction in both the grain boundaries of homo-phase regions and the mesoscopic hetero-junctions in the nanostructure. Increasing the SDC particle size from 25 to $45 \mathrm{~nm}$ caused a moderate decrease in the conductivity because the density of YSZ/SDC hetero-junctions is reduced in films with larger SDC nanoparticles.

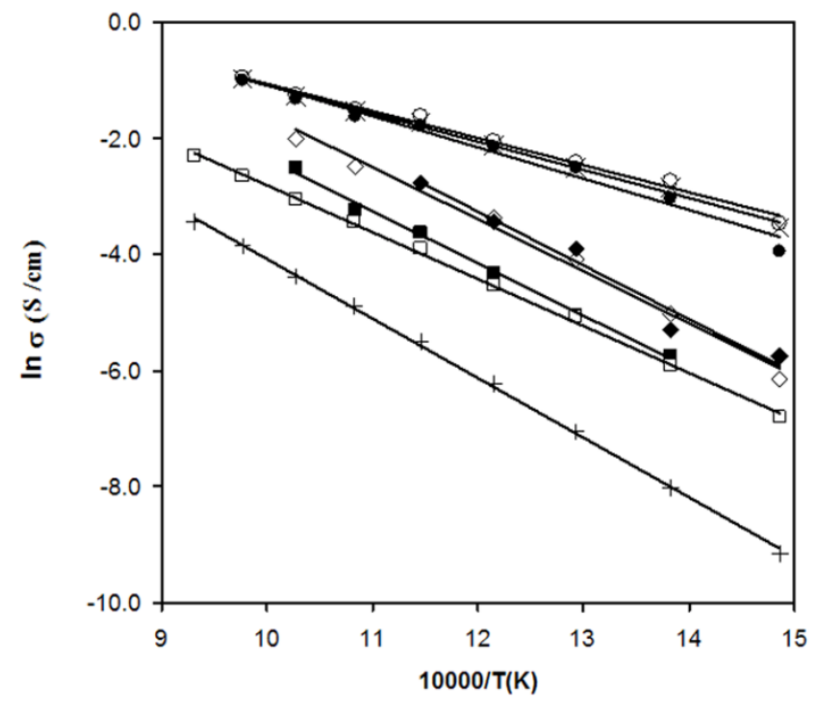

Figure 51. Electrical Conductivity as a function of temperature. $\bigcirc$ SDC800/YSZ hetero-phase film, $\times$ SDC1000/YSZ, $\bullet$ SDC1200/YSZ hetero-phase film, $\diamond$ YSZ nano-film (this work), $\bullet$ YSZ nano-film (grain size $20 \mathrm{~nm}$ ) (Ref. 10), - SDC nano-film, $\square$ SDC pellet (Ref. 8), + YSZ pellet.

Because the SDC/YSZ interface density $\left(\mathrm{m}^{2} / \mathrm{m}^{3}\right)$ in the hetero phase film depends on the SDC:YSZ volume ratio (or SDC volume fraction) and their particle sizes, additional experiments were conducted to study the effect of the SDC volume fraction on the conductivity of the heterophase film. The SDC volume fraction was varied in a range from 0.136 to 0.587 by adjusting the amount of SDC powders added to the polymeric precursors. The SDC powders fired at $800^{\circ} \mathrm{C}$ 
were used for all the films with different SDC volume fractions. The electrical conductivities of the films are presented as a function of SDC volume fraction in Figure 52, which also includes the data of phase-pure YSZ and SDC films. The highest conductivity was obtained on the film with a SDC volume fraction of 0.495 , which possessed the highest interface area among the samples studied. A SDC volume fraction of 0.68 or greater resulted in a porous structure as indicated by the reduced transparency of the film and drastically decreased conductivity.

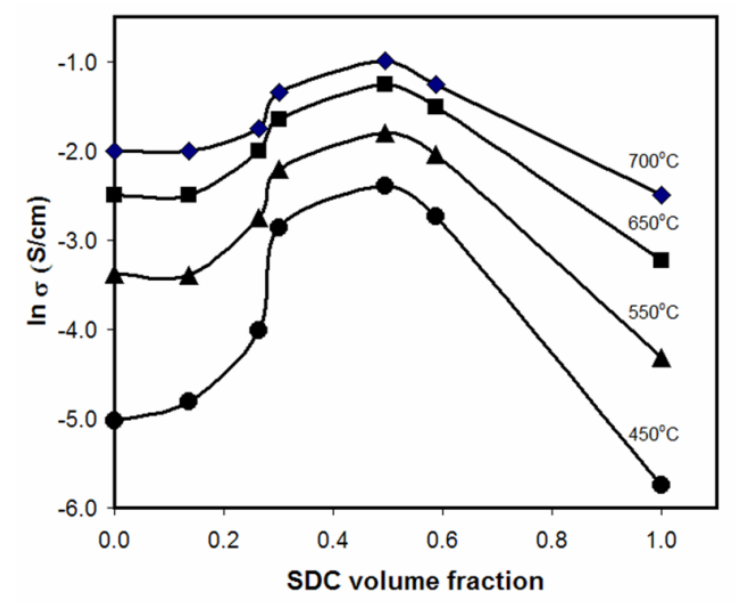

Figure 52. Electrical Conductivity as a function of SDC volume fraction at different temperatures. $\left(-450^{\circ} \mathrm{C}, \Delta 550^{\circ} \mathrm{C}, \bullet 650^{\circ} \mathrm{C}, \bullet 750^{\circ} \mathrm{C}\right)$ 


\subsection{Pd thin film coating on optical fiber by sputtering deposition}

During the sputtering deposition, the fiber was placed in the chamber with a pressure of 5mTorr. $99.9 \%$ Ar was used as the working gas. The plasma was turned off at a certain time after the deposition started depending on the film thickness required. Then, the fiber was turned 180 degree to make the other side coated with Pd also. The second step also lasted for the same amount of time. Certain gun power was applied during the deposition. After the deposition, the film quality was characterized by SEM and EDS.

\subsubsection{Pd deposition at the sputtering current of $15 \mathrm{mAmp}$}

Figure 53 a and Figure 53 b show the SEM images taken at two different areas on an optical fiber. The films are uniform with a few defects (black spot on the side surface). Figure $53 \mathbf{c}$ is the SEM image taken on the fiber end. The film looks rougher compared to the one on the body. It could be due to the uneven end cut without the appropriate tool (Later on, fibers prepared by Prof. Xiao's group using fiber cleaver will be utilized). The grain size of Pd thin was around 15-20nm as shown in Figure 53 d. 

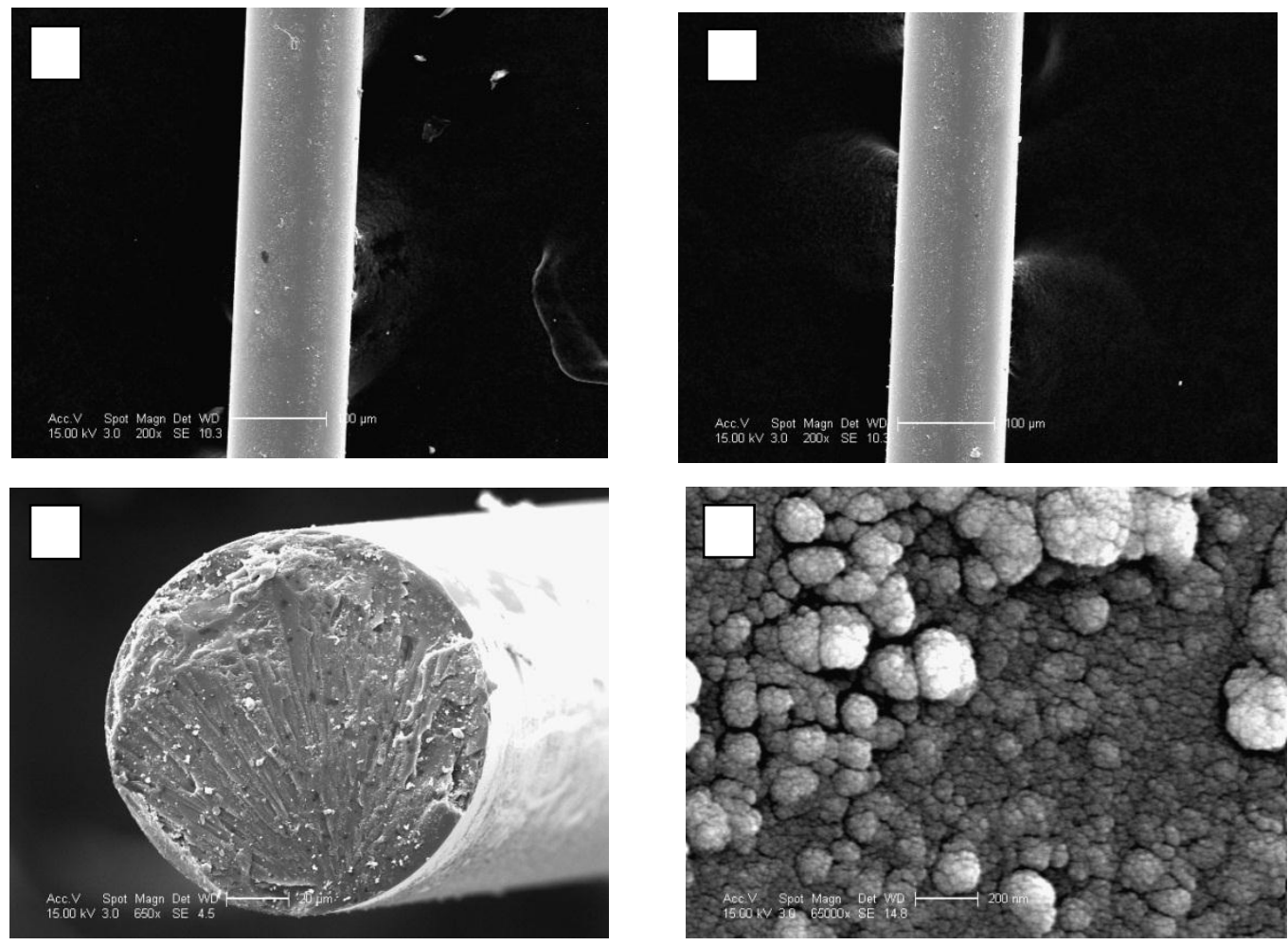

Figure 53. SEM images of Pd coated on optical fiber at the sputtering power of $15 \mathrm{mAmp}$. (a) and (b) side surface coating at different fiber sections, (c) fiber endface coating, and (d) Pt particles on fiber surface.

SEM combined with focus ion beam (FIB) was utilized to analyze the Pd film thickness. FIB was applied to mill off the Pd layer on the top so that the interface could be observed clearly by SEM. The film was uniform but had a few voids. The size of the void is much smaller than the optical wavelength. The image is shown in Figure 54. Based on this image, the film thickness is around $200 \mathrm{~nm}$. 


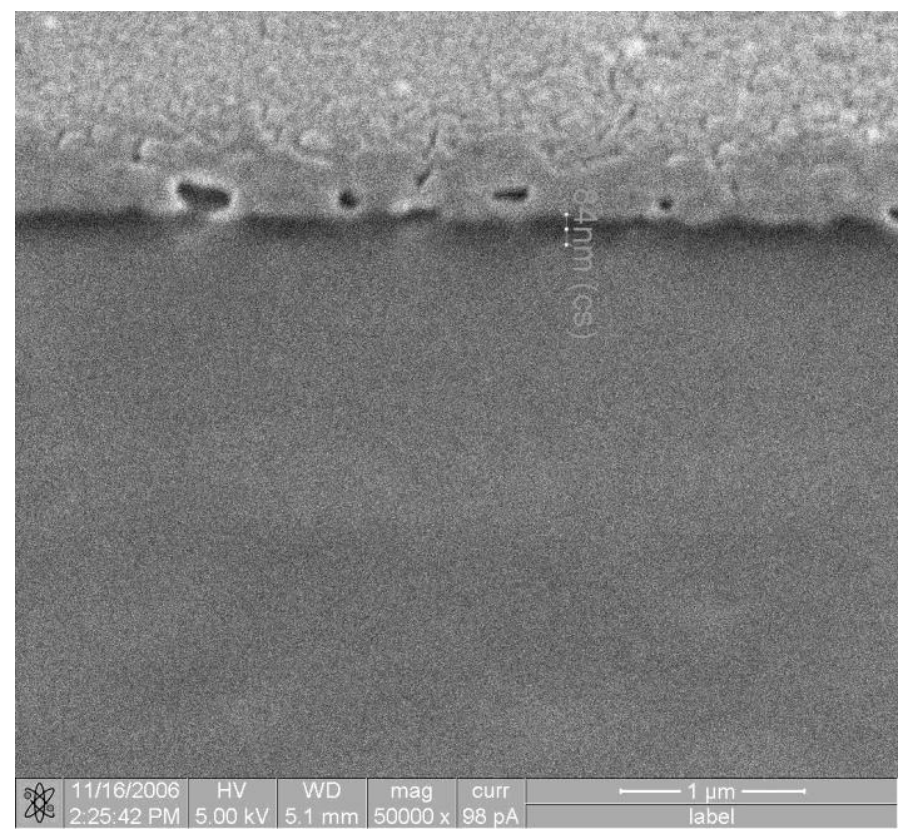

Figure 54. SEM image of $\mathrm{Pd} / \mathrm{Si}$ fiber interface

EDS was applied in analyzing the composition of the film coated on the fiber side surface and end face. The uniformity of the film was estimated at six different spots along $1 \mathrm{~cm}$ long fiber section. These spots were selected randomly. The data is shown in Table 1. Composition analysis on fiber side surface sputtered at $15 \mathrm{mAmp}$. As seen, the composition difference among these areas is quite small, which indicates the good uniformity of the Pd film. 
Table 1. Composition analysis on fiber side surface sputtered at 15mAmp

\begin{tabular}{|c|l|l|l|l|l|l|}
\hline Element & Spot 1 & Spot 2 & Spot 3 & Spot 4 & Spot 5 & Spot 6 \\
\hline $\mathrm{Si}$ & 30.83 & 30.95 & 29.27 & 28.15 & 32.31 & 34.12 \\
\hline $\mathrm{wt} \%)$ & 69.17 & 69.05 & 70.73 & 71.85 & 67.69 & 65.88 \\
\hline $\mathrm{wt} \%)$ & & & & & & \\
\hline
\end{tabular}

Table 2 lists the composition on the coated fiber end face, where five different sites were chosen randomly. Compared to the Pd content on the side surface, the end face had less amount of $\mathrm{Pd}$. We believe that this is mainly due to the orientation of which the fiber placed in the puttering chamber. It could also be caused by the coarse quality of the fiber cut.

Table 2. Composition analysis on fiber end sputtered at 15mAmp

\begin{tabular}{|c|c|c|c|c|c|}
\hline Element & Spot 1 & Spot 2 & Spot 3 & Spot 4 & Spot 5 \\
\hline $\mathrm{Si}$ & 72.37 & 62.48 & 84.20 & 70. & 69.59 \\
$(\mathrm{wt} \%)$ & & & & 12 & \\
\hline $\mathrm{Pd}$ & 27.63 & 37.52 & 15.80 & 29. & 30.41 \\
\hline$(\mathrm{wt} \%)$ & & & & 88 & \\
\hline
\end{tabular}




\subsubsection{Pd deposited at the sputtering current of 9mAmp}

Similarly, Figure 55. SEM images of Pd coated on optical fiber at the sputtering power of 9mAmp. shows the SEM images of Pd film sputtered on fiber side surface and end face at the current of 9mAmp. Compared to the one sputtered at a higher current (Figure 55), more defects occurred on this film. Because the film on the end was so thin, the SEM image on the end is not clear due to the charging effect. The grain size shown in Figure $\mathbf{5 5} \mathrm{d}$ is in the range of 15-20nm.
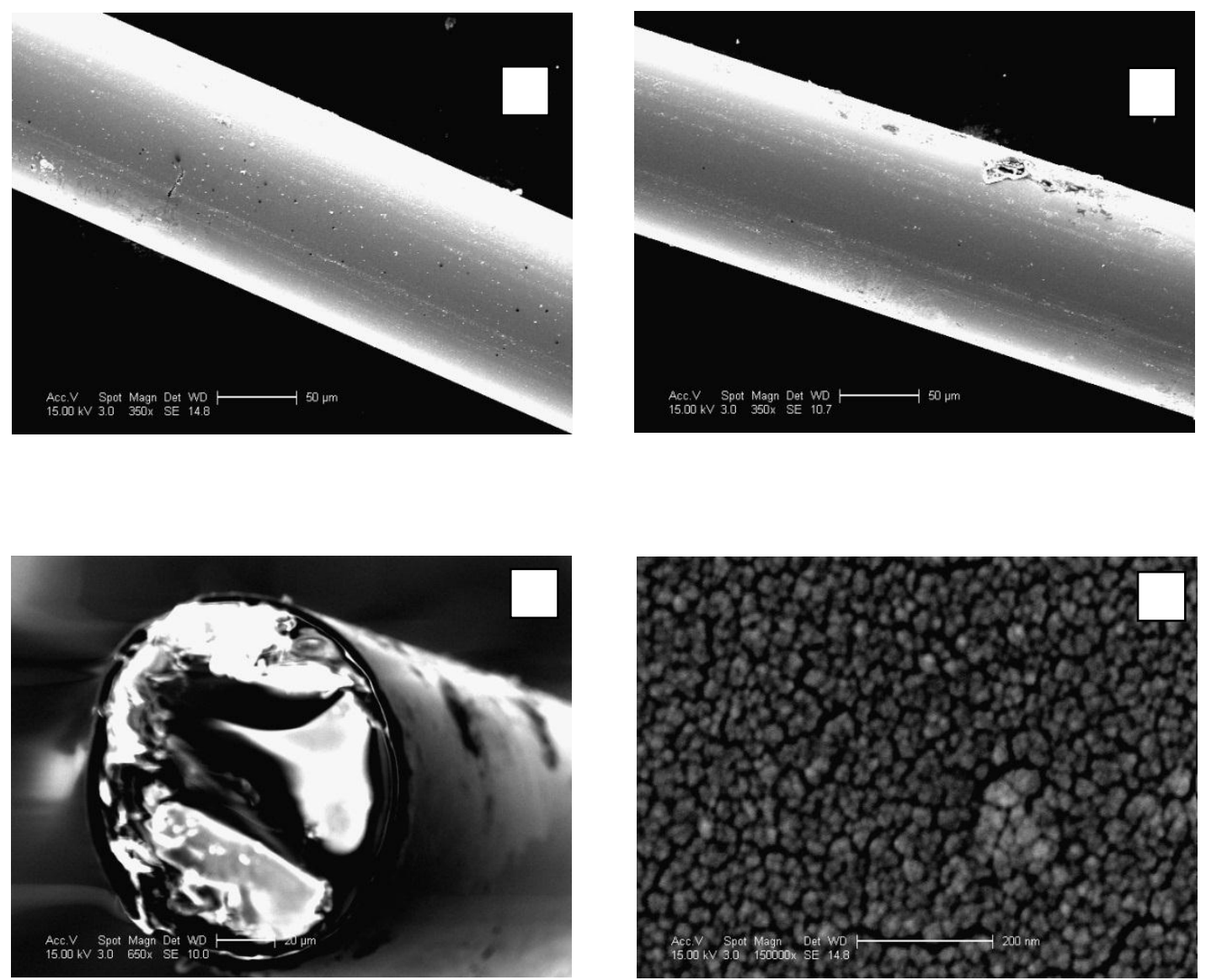

Figure 55. SEM images of Pd coated on optical fiber at the sputtering power of 9mAmp. (a) and (b) side surface coating at different fiber sections, (c) fiber endface coating, and (d) pt grain size. 


\subsubsection{Pd thin film coating on optical fiber by electroless plating}

Pd deposition by electroless plating onto optical fibers was investigated. This method consists of two steps namely activation, and electroless plating. An optical fiber with its polymer buffer stripped was immersed into 0.2M Pd acetate solution. Once dried and reduced in hydrogen at $500^{\circ} \mathrm{C}$, the $\mathrm{Pd}$ deposition was carried out overnight. We found that the film prepared by electroless plating was quite thick and not uniform. Some Pd layer even peeled off during the deposition process. Two microscopic optical images of Pd/optical fiber after electroless plating are shown in Figure 56. In an effort to find out the reason for the formation of low quality film, Pd thin film was deposited on flat $\alpha$-alumina support by electroless plating. The SEM image is shown in

Figure 57. As seen, the Pd film has the grain size distributed at around 200nm, which is almost equal to the film thickness required on the fiber. The poorer film quality was thus attributed to the larger grain size.
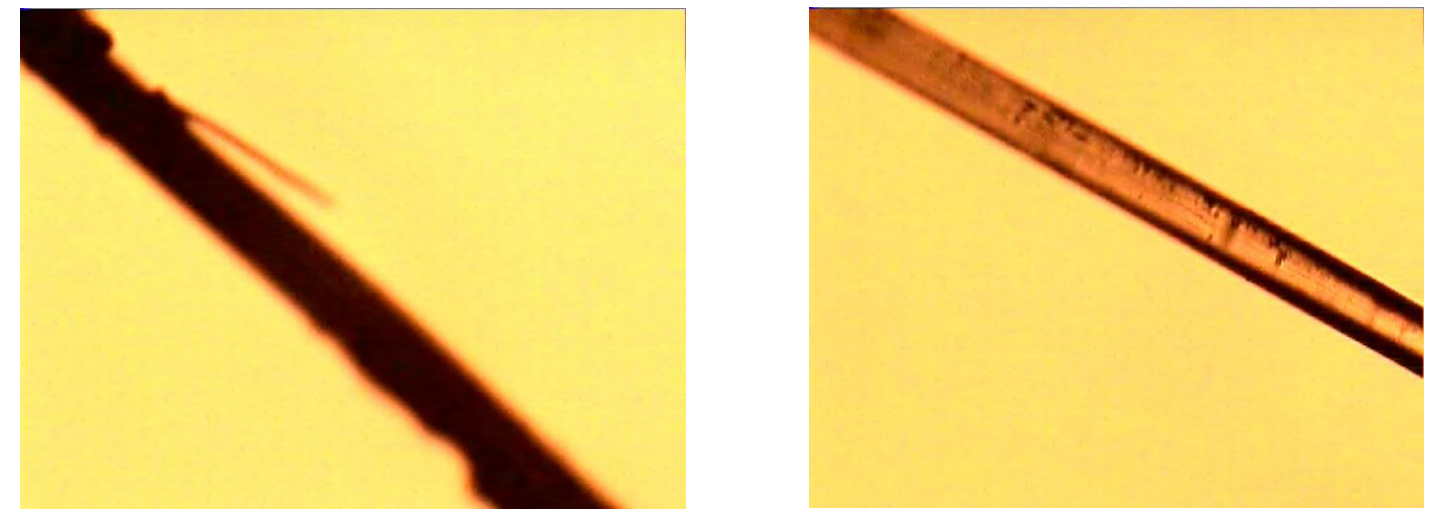

Figure 56. Microscopic optical images of pd-coated optical fiber by electroless plating 


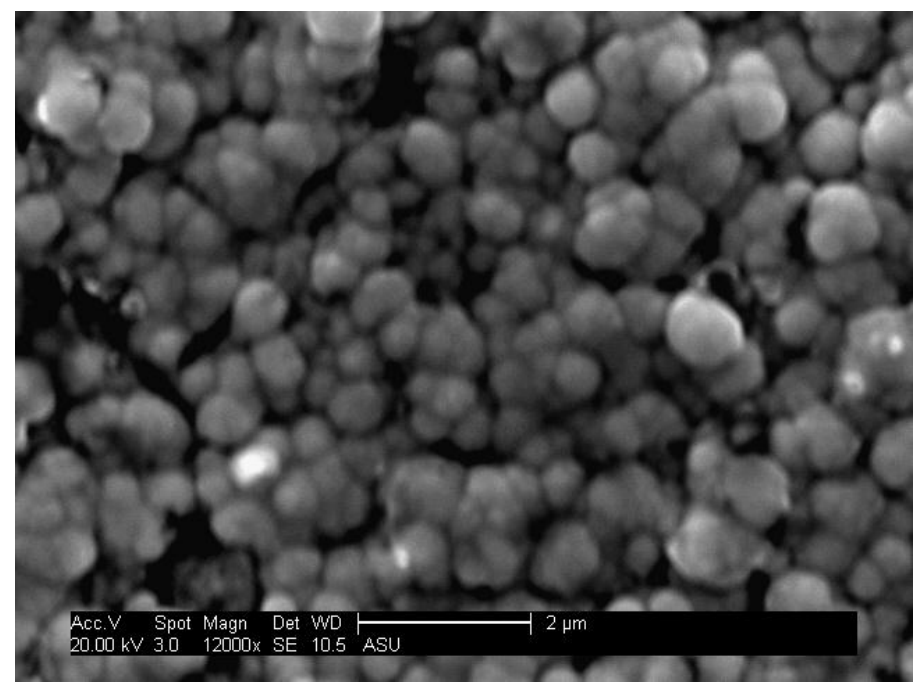

Figure 57. SEM image of Pd particles on optical fiber by electroless plating

\subsubsection{Grain size modification by thermal treatment}

Pd-Cu was prepared by sputtering deposition. The composition was characterized by EDS. The foil contains $58 \% \mathrm{Pd}$ and $42 \% \mathrm{Cu}$. Samples were annealed at $500^{\circ} \mathrm{C}$ for a period of 3.5, 6 and 10 hours and $800^{\circ} \mathrm{C}$ for 18 hours, respectively. Table 3 shows the data for estimated grain size.

The XRD patterns of untreated and annealed samples are shown in Figure 58. As seen in the results, without thermal treatment, the sample contains a predominant fcc phase. The phase diagram shows that the stable phase for the $\mathrm{Pd}-\mathrm{Cu}$ alloy this composition is of bcc structure. This suggests that the sputter deposited $\mathrm{Pd}-\mathrm{Cu}$ is at the unstable fcc structure. All samples after the thermal treatment exhibit the bcc phase. This is because annealing at a high temperature allows 
Pd-Cu to convert from the unstable fcc structure to the stable bcc structure.

Table 3 Estimated grain size of $\mathrm{Pd}-\mathrm{Cu}$ annealed under different conditions

\begin{tabular}{|c|c|}
\hline Sample & Grain size \\
\hline Without annealing & Too small to be characterized by SEM \\
\hline $500^{\circ} \mathrm{C} 6$ hours & $250 \mathrm{~nm}$ \\
\hline $500^{\circ} \mathrm{C} 12$ hours & $250 \mathrm{~nm}$ \\
\hline $800^{\circ} \mathrm{C} 18$ hours & $800 \mathrm{~nm}$ \\
\hline
\end{tabular}

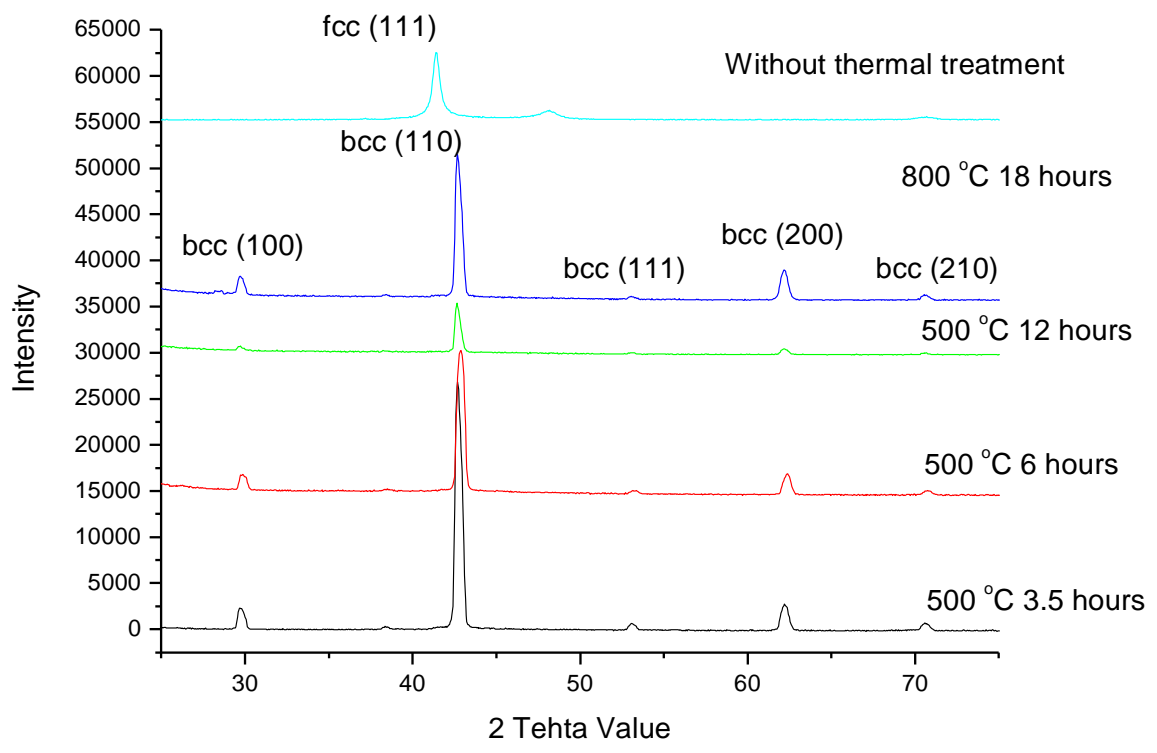

Figure 58. XRD patterns of $\mathrm{Pd}(58 \%)-\mathrm{Cu}$ alloy before and after annealing 
Figure 59 shows the SEM images of the Pd-Cu alloy at different annealing conditions. After annealed at $500^{\circ} \mathrm{C}$, the apparent increase in the grain size was observed. Increase in the annealing time at $500^{\circ} \mathrm{C}$ did not have significant effect on the grain size growth. The sample annealed at $800^{\circ} \mathrm{C}$ for 18 hours had the largest grain size compared to other ones. These results indicate that samples with different grain sizes could be obtained by thermal treatment under controlled conditions. Moreover, the change of structure could be avoided.
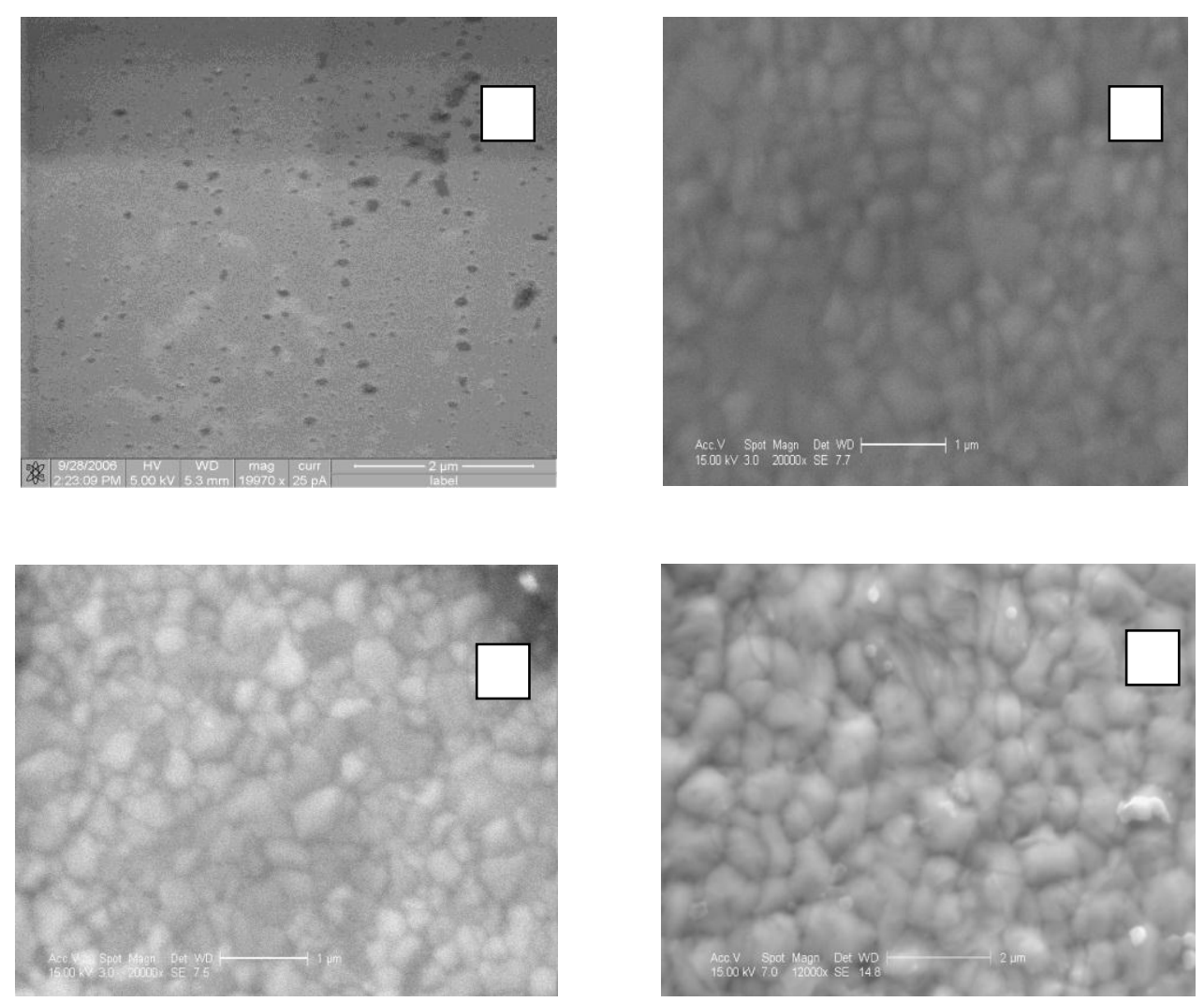

Figure 59. SEM images of Pd-Cu alloy (a) without treatment, (b) annealed at 500oC for 6 hours, (c) 12 hours and (d) annealed at $800 \mathrm{C}$ for 18 hours. 


\section{$\underline{\text { 2.4.5 Refractive index of Pd thin film deposited on dense alumina disk }}$}

Ellipsometer was utilized to measure the refractive index of Pd thin film by sputter deposited on dense alumina disk. The result is shown in the Figure 60. In the Figure, the black line shows refractive index (n) and the blue line shows the extinction coefficient (k).

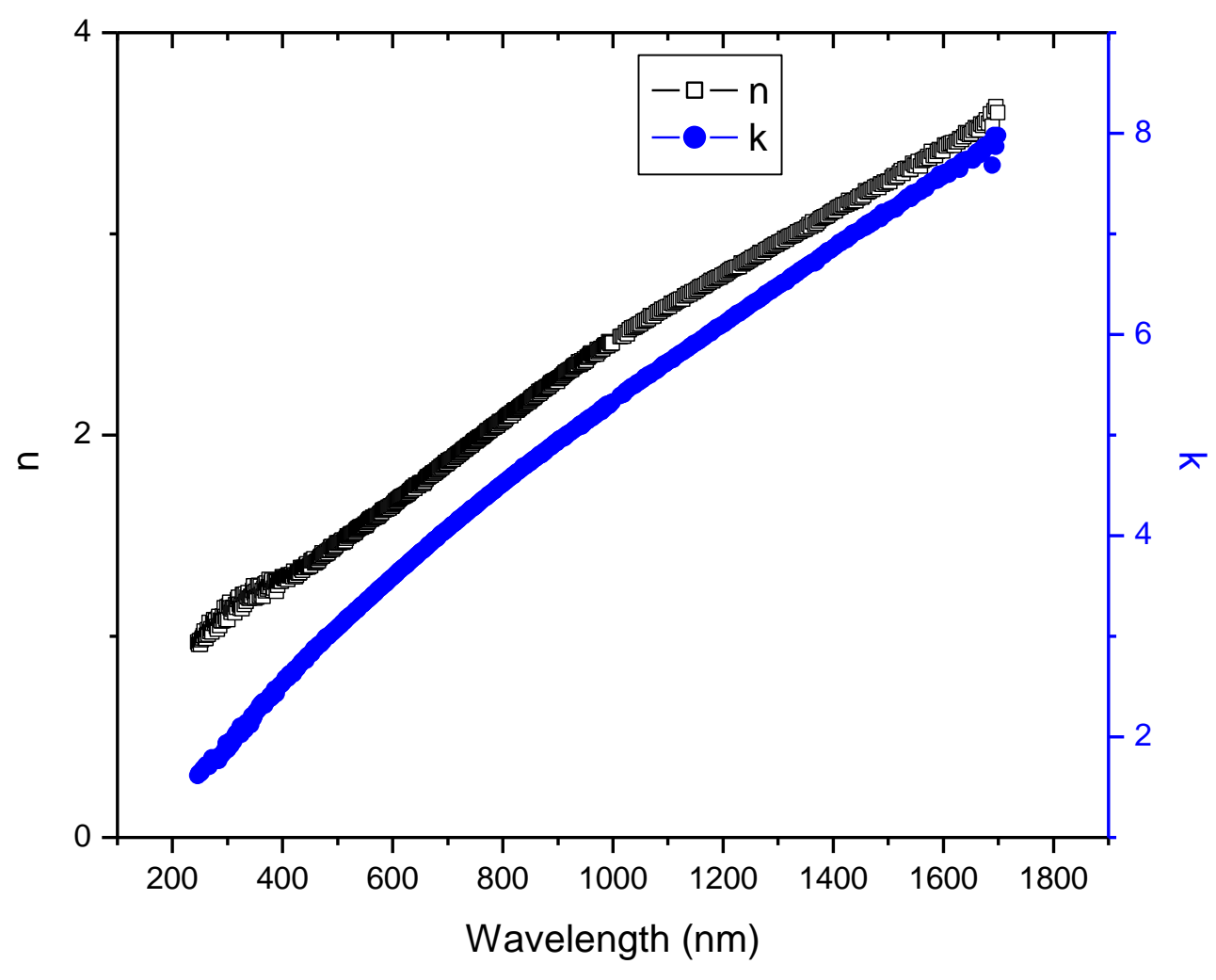

Figure 60. $\mathrm{n}, \mathrm{k}$ of Pd thin film deposited on alumina bar measured by ellipsometer

The data was compared to the one for bulk Pd reported by P. B. Johnson and R. W. Christy [Johnson P. B. and Christy R. W. "Optical constants of transition metals: Ti, V. Cr, Mn, Fe, Co, Ni and Pd", Phys. Rev. B 9 (1974) 9] and is listed in Error! Reference source not found.. As seen in the table, the refractive index acquired was higher than the one reported in the literature. 
Table 4. Comparison of refractive index of Nano - crystalline Pd and bulk Pd

\begin{tabular}{|c|c|c|c|c|}
\hline \multirow{2}{*}{$\mathrm{eV}$} & $\mathrm{n}$ & $\mathrm{k}$ & $\mathrm{n}$ & $\mathrm{k}$ \\
\hline 0.77 & 3.45 & 7.63 & 3.01 & 8.59 \\
\hline 1.64 & 1.99 & 4.32 & 1.95 & 4.89 \\
\hline 2.50 & 1.45 & 3.07 & 1.52 & 3.54 \\
\hline 3.25 & 1.23 & 2.40 & 1.26 & 2.83 \\
\hline 4.24 & 1.08 & 1.84 & 1.18 & 2.23 \\
\hline 4.98 & 0.98 & 1.64 & 1.08 & 1.99 \\
\hline
\end{tabular}




\subsection{Synthesis of silicalite over-coat on LPGF}

Pure silica MFI zeolite (silcalite) overcoat was successfully synthesized on the LPGF provided by Xiao's group. SEM examination has indicated that the zeolite overcoat has a dense layer on the fiber surface although the outer surface shows discontinuous zeolite crystal coverage. The crystals in the continuous layer (at zeolite/fiber interface) were primarily boriented as shown in Figure $\mathbf{6 1}$ below. The zeolite overcoat synthesis and zeolite activation processes were continuously monitored measuring the optical spectrum. The results indicated that the same monitoring approach can be utilized to assist the coating of nano crystalline ceramic films for high temperature gas sensing. The zeolite-LPGF structure was also tested to be thermally stable at $550^{\circ} \mathrm{C}$.
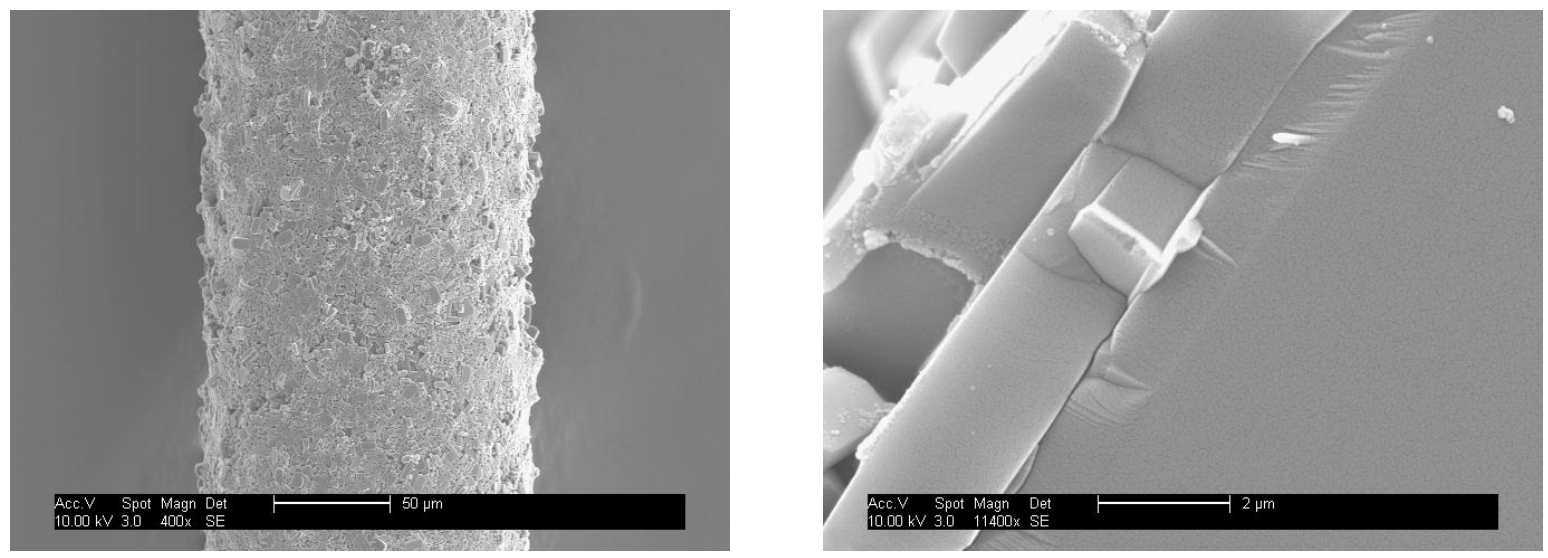

Figure 61. SEM images of the silicalite coated LPGF (left: surface; right: cross-section). 


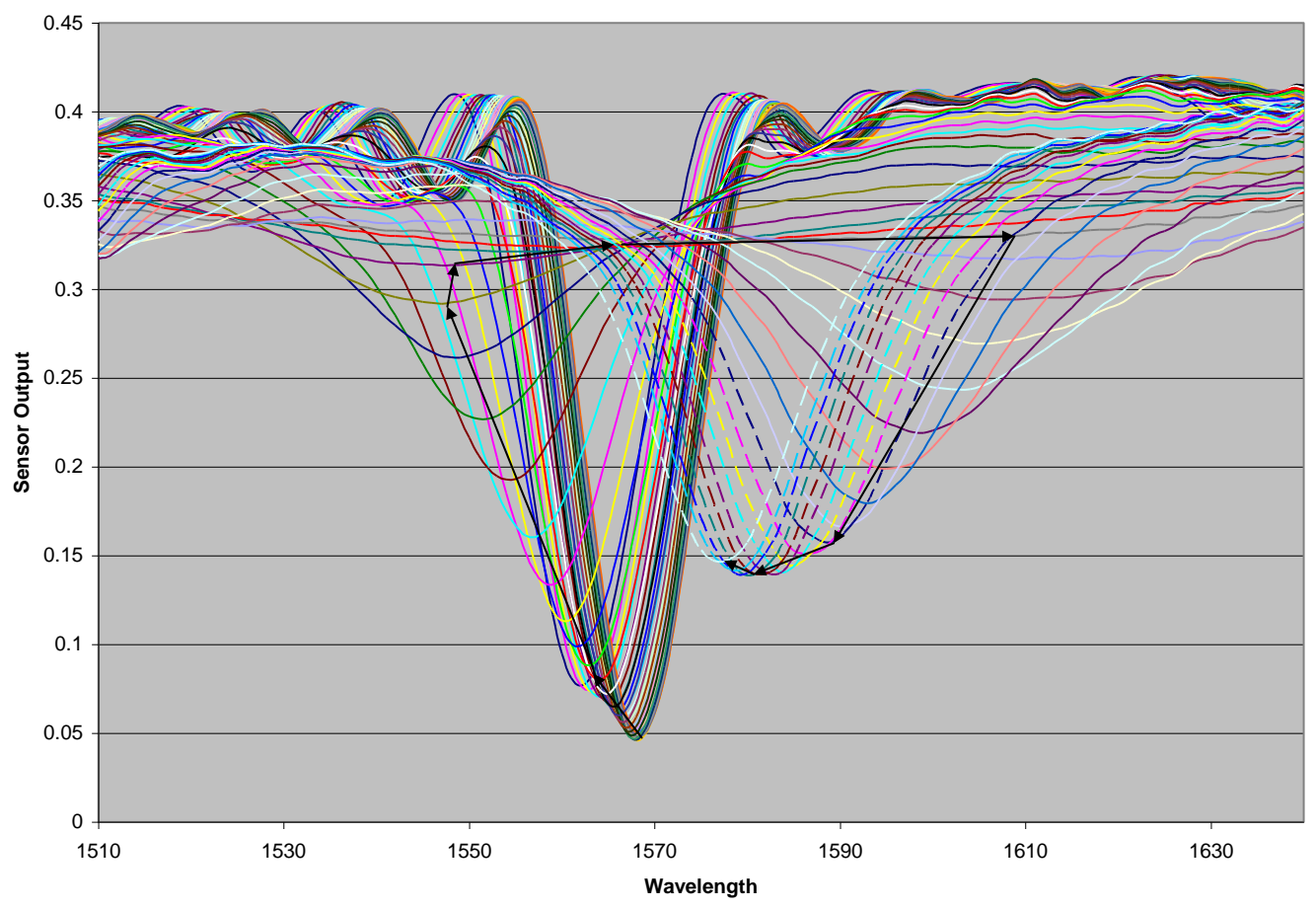

Figure 62. Monitoring of the zeolite overcoat synthesis process

\subsubsection{Test of LPGF sensor for detecting chemical vapors}

For demonstration of the general sensing mechanism of the proposed nanomaterial-LPGF sensors, the silicalite-LPGF was tested for detection of isopropanol and toluene vapors. Figure 63 shows the results of the measurements. The LPGF show very high sensitivity for chemical vapor detection. For example, a $0.7 \mathrm{~nm}$ shift of the responding (notch) wavelength was obtained for a toluene vapor concentration (in nitrogen) of 220 parts per billion (ppb) when the resolution of the LPGF sensor can be at $0.01 \mathrm{~nm}$. The correlation between the toluene concentration and the sensor output signal is presented in Figure 63. Results of the zeolite LPGF sensor for 
detection of trace chemical vapors. These results prove that the proposed ceramic nanomaterial-coated LPGF sensors will possess the sensitivity and resolution for measurement of gas composition in a wide range of concentration.

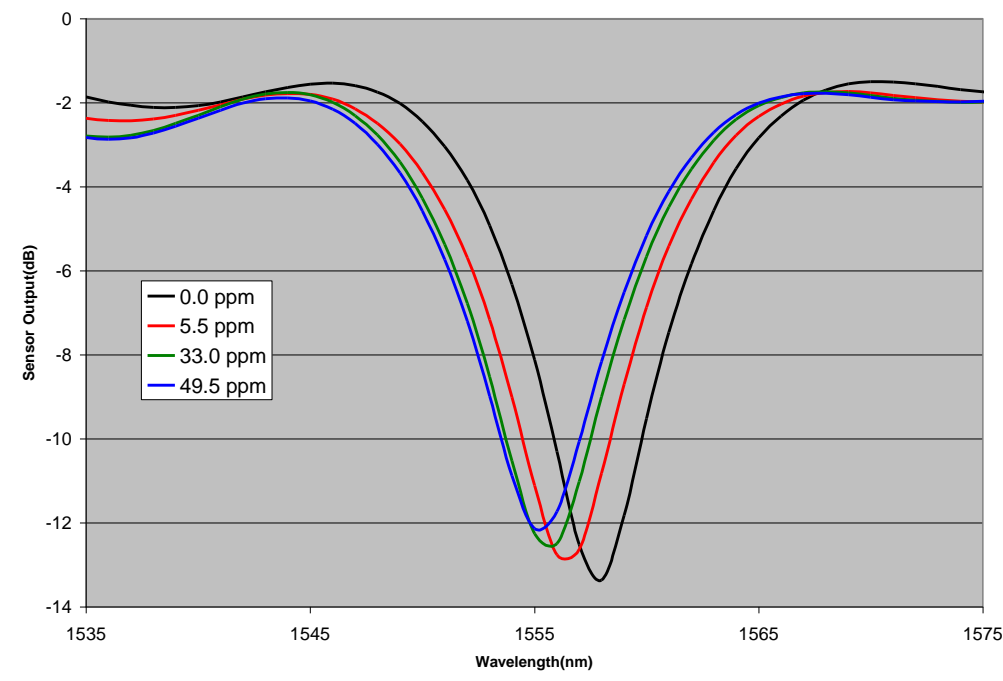

(a) Results of sensor response to isopropanol vapor in a range of $0-50 \mathrm{ppm}$.

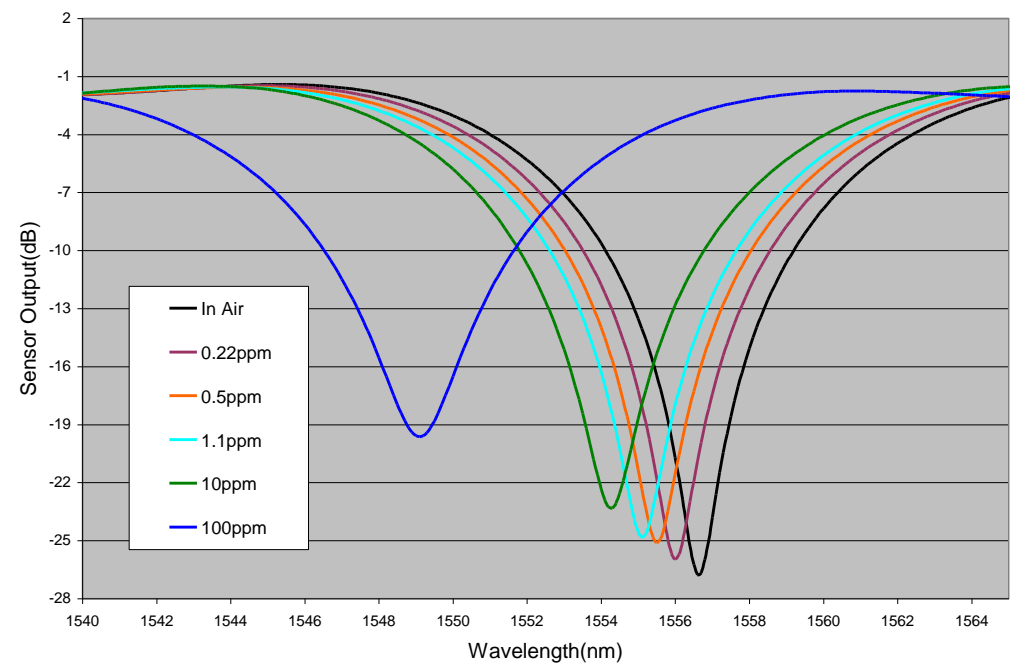

(b) Results of sensor response to toluene vapor in a concentration range of $0-100 \mathrm{ppm}$.

Figure 63. Results of the zeolite LPGF sensor for detection of trace chemical vapors. 


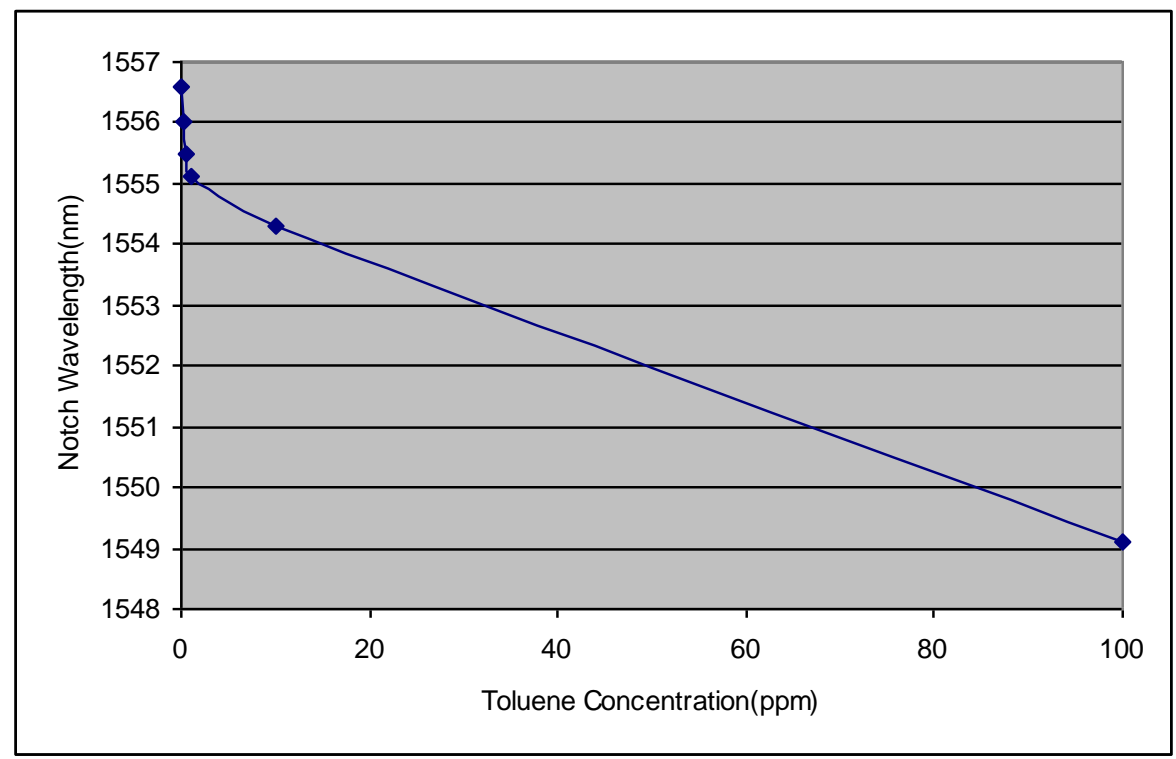

(a) Notch wavelength as a function of toluene concentration

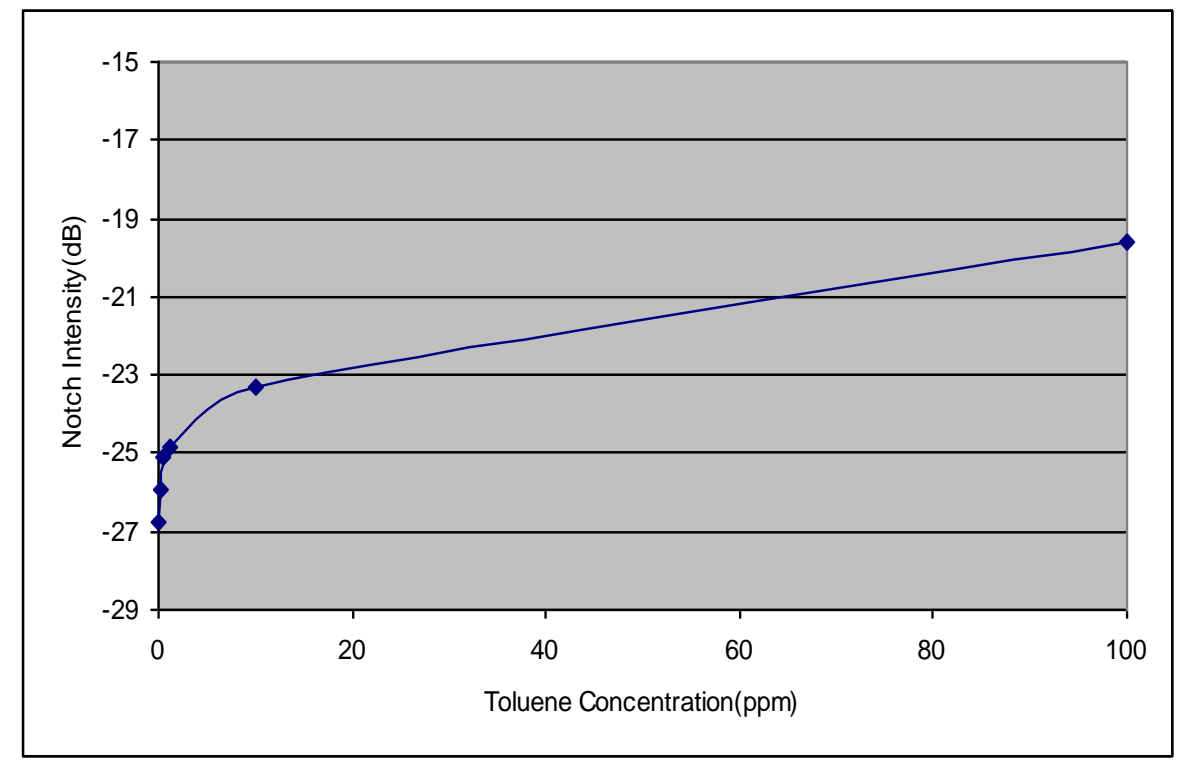

(b) Notch intensity as a function of toluene concentration

Figure 64. Correlations between the sensor output signals and the toluene concentration 


\section{$\underline{2.5 .1 \mathrm{ZrO}_{2}} / \underline{\mathrm{CuO} \text {-LPFG Sensor fabrication and characterization }}$}

Nano-crystalline $\mathrm{ZrO}_{2} / \mathrm{CuO}$ composite thin films $(120-150 \mathrm{~nm}$ thick $)$ have been synthesized on LPFG cylindrical surface. The crystallite size of the metal oxide was estimated to be $20-30 \mathrm{~nm}$ according to XRD and HRSEM examinations. Figure 65 shows the microscopic images and EDS elementary analysis results of the $\mathrm{ZrO} 2 / \mathrm{CuO}-\mathrm{LPFG}$ sensor.
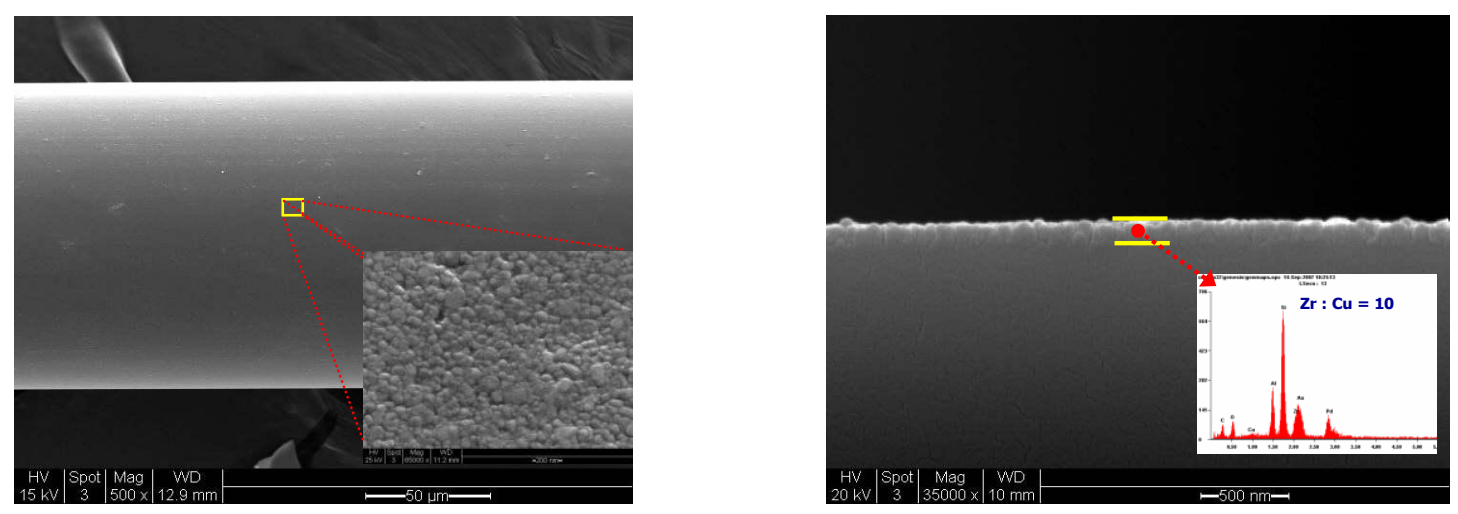

Figure $65 \mathrm{SEM}$ pictures of the $\mathrm{ZrO}_{2} / \mathrm{CuO}-\mathrm{LPFG}$ sensor 


\section{$\underline{\text { 2.5.2. Test of } \mathrm{ZrO}_{2}} \underline{2 \mathrm{CuO}-\mathrm{LPFG} \text { sensor for low concentration } \mathrm{CO} \text { monitoring in } \mathrm{CO}_{2}}$}

The $\mathrm{ZrO}_{2} / \mathrm{CuO}-\mathrm{LPFG}$ sensors were tested for $\mathrm{CO}$ detection in $\mathrm{CO}_{2}$ at $550^{\circ} \mathrm{C}$ and atmospheric pressure for more than $250 \mathrm{hr}$. The sensor is capable of detecting CO concentration in a range from 0 to $\sim 10,000 \mathrm{ppm}(1 \%)$ with excellent senitivity but had problems with reversibility at higher $\mathrm{CO}$ concentrations $(>0.5 \%)$. Figure $\mathbf{6 6}$ presents the sensor output (i.e. resonant wavelength) as a function of $\mathrm{CO}$ concentration in $\mathrm{CO}_{2}$. The sensor's speed of measurement (i.e. response time) was found to be relatively long (six minutes).

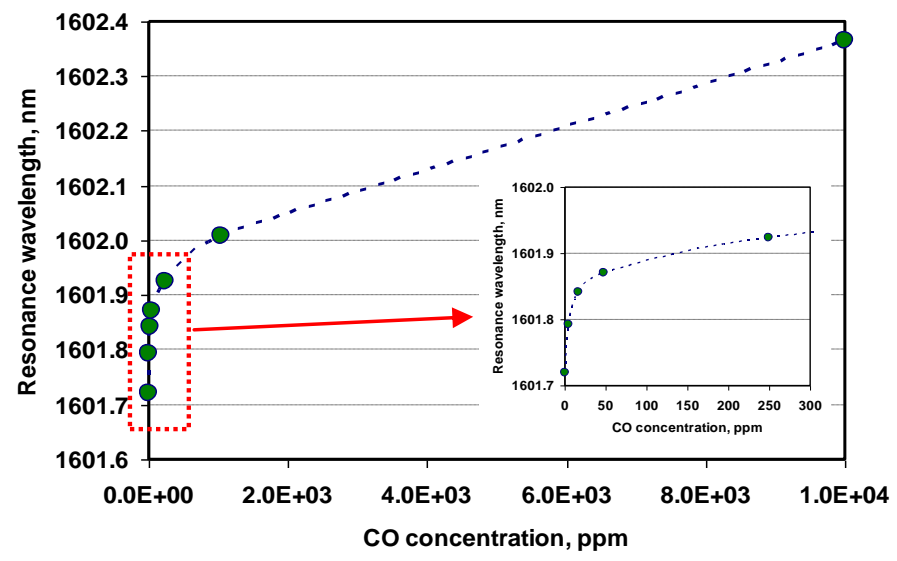

Figure 66 Resonant wavelength as a function of $\mathrm{CO}$ concenration in $\mathrm{CO}_{2}$ at $550^{\circ} \mathrm{C}$ and $1 \mathrm{~atm}$. 


\section{$\underline{\text { 2.5.3 MFI zeolite-LPFG sensors fabrication and characterization }}$}

Figure 67 shows the microscopic images of the MFI zeolite-LPFG (MFI-LPFG) sensor. The MFI zeolite coating was synthesized from an aluminum-free synthesis solution and activated by calcination at $550^{\circ} \mathrm{C}$ in air for $4 \mathrm{hr}$.
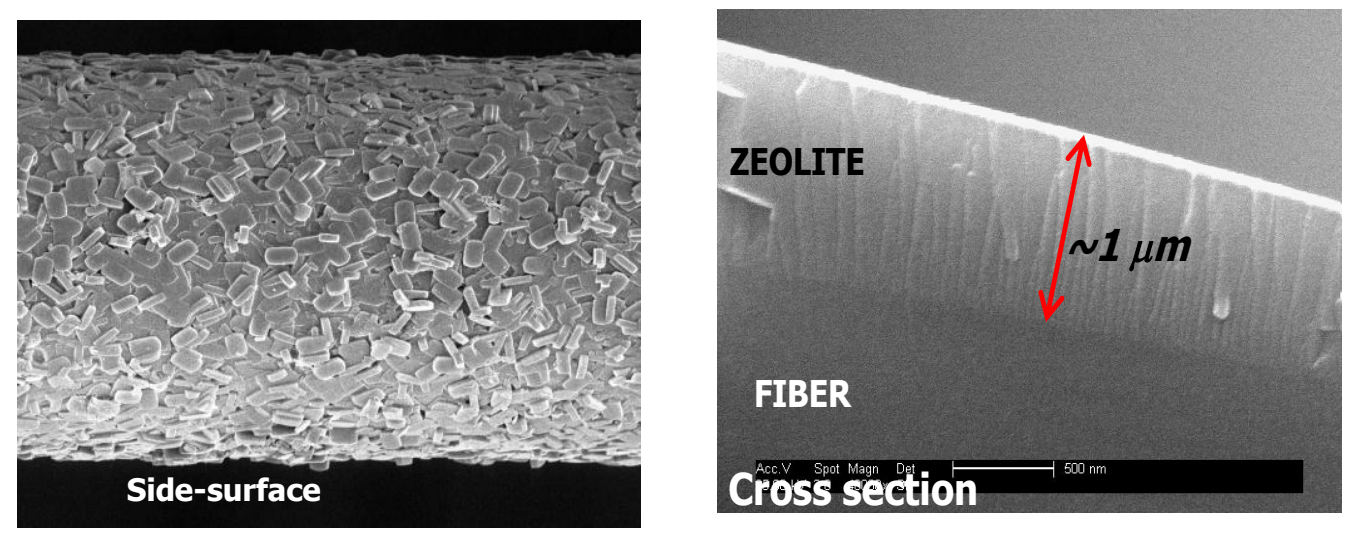

Figure 67 SEM images of the MFI-LPFG sensor 


\subsubsection{Test of the MFI-LPFG sensor for bulk $\mathrm{H}_{2}$ measurement in $\mathrm{N}_{2}$}

Figure 68 presents the results of measurements of bulk $\mathrm{H}_{2}$ concentration in $\mathrm{N}_{2}(10-33 \%$ $\mathrm{H}_{2}$ ) The sensor response time was short (less than three seconds). The experiments were performed at $400^{\circ} \mathrm{C}$ and atmospheric pressure due to the limit of gas supply system. Because the sensor works on a "P-V-T" mechanism, it is likely that similar or better sensitivity will be achieve when applying to high pressure gases. In addition, we did not test at higher temperatures in order to minimize the interference from the thermal drafting of the fiber grating.

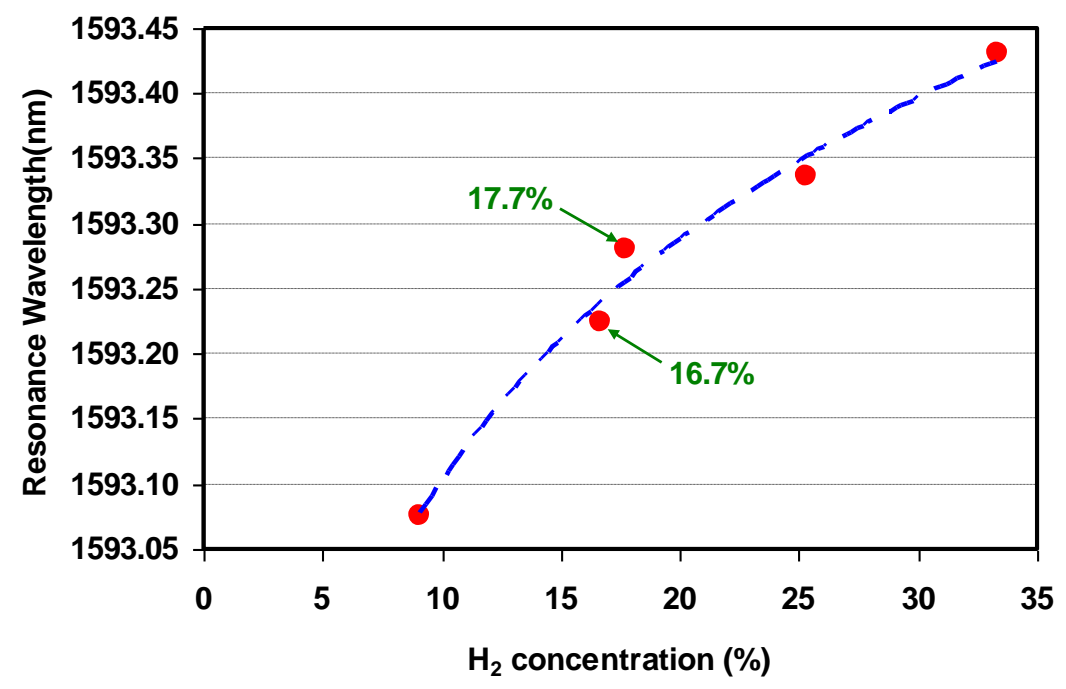

Figure $68 \mathrm{MFI}-\mathrm{LPFG}$ sensor response to $\mathrm{H} 2$ concentration in $\mathrm{N}_{2}$ at $400^{\circ} \mathrm{C}$.

Figure 69 presents the results of responses time to concentration (or gas density) change on an end face sensor (single wavelength measurement). Since both the LPFG and end face sensor response times are determined by the same diffusion-controlled equilibrating time, the 
LPFG sensor is expected to have similar response speed.

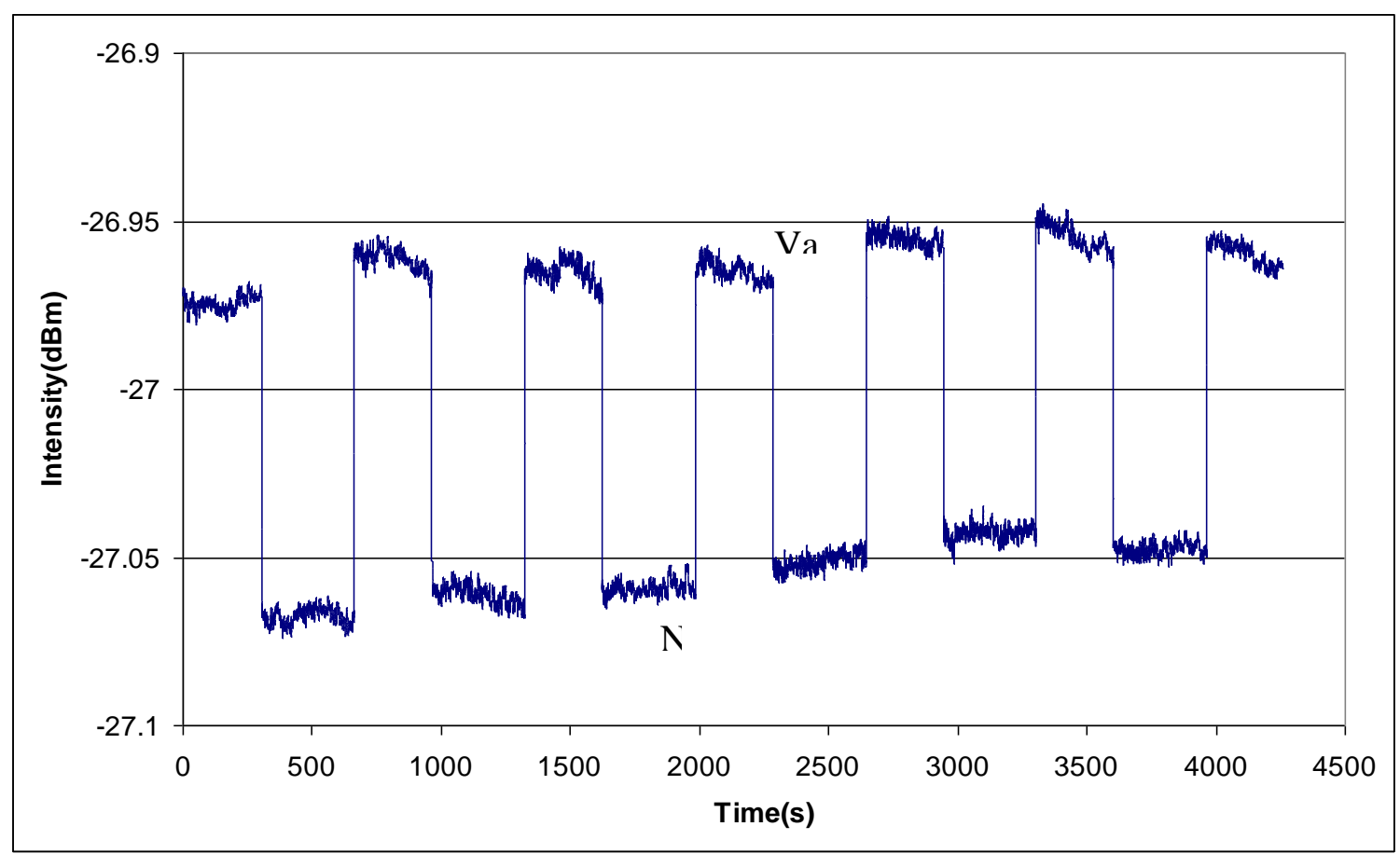

(a). Switch between $\mathrm{N}_{2}(107 \mathrm{kPa})$ and Vacuum( $(9 \mathrm{kPa}), \lambda=1578 \mathrm{~nm}$ 


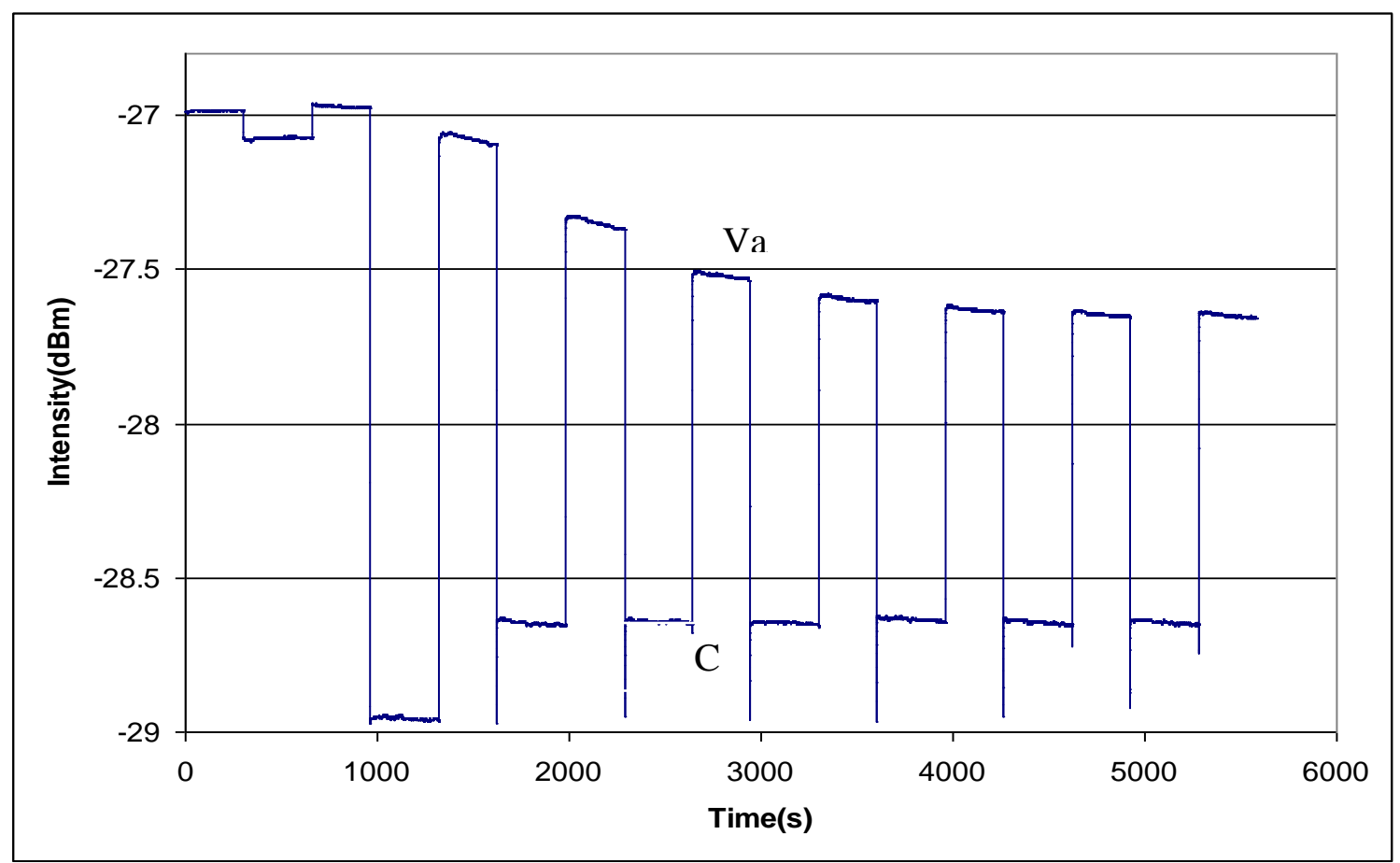

(b). Switch between $\mathrm{CO}_{2}(112 \mathrm{kPa})$ and Vacuum $(9 \mathrm{kPa}), \lambda=1578 \mathrm{~nm}$

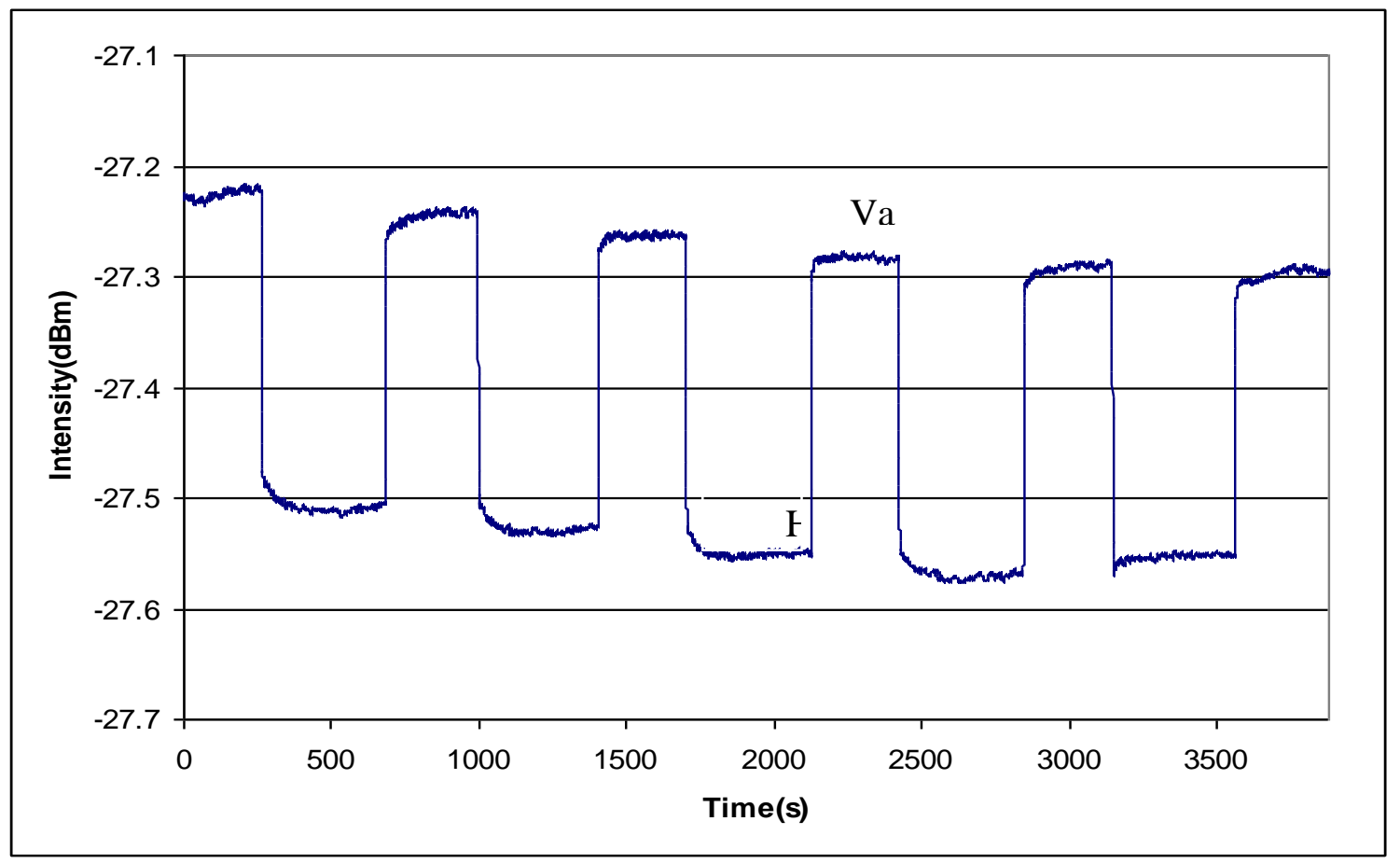

(c). Switch between $\mathrm{H}_{2}(134 \mathrm{kPa})$ and Vacuum $(9 \mathrm{kPa}), \lambda=1588 \mathrm{~nm}$ 
Figure 69 Sensor response to gas switching

\subsubsection{Organic Vapor Detection}

The MFI zeolite-LPFG sensor was tested for detection of iso- propanol (HPLC 99.9\%, Acros) and toluene (HPLC >99.9\%, Aldrich) vapors in air. The measurements were conducted at room temperature under atmospheric pressure for low concentrations of organic vapors. For both iso-propanol and toluene vapors, increasing the vapor concentration caused the $\Delta \mathrm{R}$-shift toward smaller wavelength. Such a shift of $\Delta \mathrm{R}$ is resulted from the increase of zeolite film refractive index induced by the increasing sorption of organic molecules in the zeolite cavity. The amount of analyte sorption increases as its concentration increases in the atmosphere. This observation is well explained by the three-layer model.

The $\Delta \mathrm{R}$ shifted $1.45 \mathrm{~nm}$ for an iso-propanol vapor concentration of $5.5 \mathrm{ppm}$ and shifted as much as $0.7 \mathrm{~nm}$ for a toluene vapor concentration of $0.22 \mathrm{ppm}$ (i.e. $220 \mathrm{ppb}$ ). The sensitivity and lower limit of the measurement depend on the type of analyte molecule.

For the same concentration, the amount of molecular sorption, sorbate-framework interaction, and molecular sorption-induced changes in the zeolite structure and density are different for molecules with different chemical properties such as mass and polarity. These analyte-specific adsorbing behaviors affect the magnitude of variations in the zeolite refractive index and optical thickness. It should be noted that the lowest concentration tested in this study was constrained by the sample gas generation technique used but not the limit for sensor detection. With today's technical ability to detect $1 \mathrm{pm}$ wavelength displacement, it is anticipated that the sensor's limit of detection for toluene would be at least in the range of lower ppb level.

Results of this research demonstrate that zeolites are excellent materials for coating LPFG to 
develop highly sensitive sensors for quantitative measurement of trace organic vapors in gas phases. In the zeolite-LPFG sensor, the zeolite film is multifunctional, not only generating optical signals upon contacting the analyte gas but also effectively collecting/concentrating analyte molecules from trace level gas mixtures. In the past decades, a large number of synthetic zeolitic materials, including the crystalline micro porous alumino-silicates and their structural analogs of pure silica, borosilicates and alumino-phosphates, have been developed with pore sizes ranging from $0.3 \mathrm{~nm}$ to a few nanometers. The concept of physically and functionally integrating zeolite films with the LPFG may offer new opportunities to develop a variety of chemical sensors for detection of chemical and biological molecular species with high sensitivities. There are significant opportunities for the zeolite-LPFG sensors to achieve high selectivity as well. It is well known that the zeolite surface chemistry and adsorbing properties can be finely tuned by ion exchange, surface functionalization, and pre-loading of metal clusters and molecular species. The optical detection mechanism of the LPFG sensors has the advantages such as operation simplicity, electromagnetic immunity, low backward reflection, and compactness, which are desirable for portable device applications. 


\section{6 $\mathrm{Tb}$ doped $\mathrm{SrCeO}_{3}$ (SCTb) Hydrogen sensing material Development and}

\section{Characterization}

Hydrogen sensing by SCTb coated LPFGs was studied at $600^{\circ} \mathrm{C}$. In the first test, when the gas environment was switched from $\mathrm{He}$ to a mixture containing $4 \% \mathrm{H}_{2}$, the resonance wavelength of the LPFGs started increasing as shown in Figure 70. The resonance wavelength could not stabilize after a long period of time. The results indicate that SCTb coated LPFGs can detect $\mathrm{H}_{2}$ at $600^{\circ} \mathrm{C}$. However, the resonance wavelength can not be used as the sensing signal.

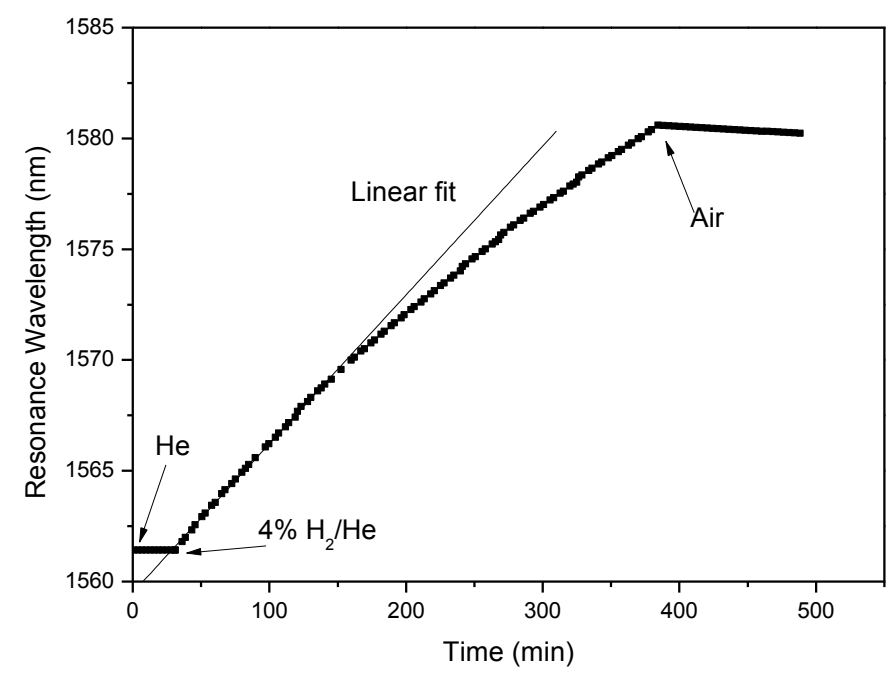

Figure 70 Resonance wavelength of SCTb-LPFGs as a function of time at $600^{\circ} \mathrm{C}$ under different gas environments.

Based on Figure 70, one can see that the resonance wavelength shift rate stays constant during the initial 100 mins. Therefore, in the second test, the effect of hydrogen partial pressure on the resonance wavelength increase rate was studied. As seen in Figure 71, the resonance wavelength increases faster under higher hydrogen partial pressure. The resonance wavelength 
increase rate as a function of hydrogen partial pressure is shown in Figure 72. This secondary data can be used as the sensing signal to monitor hydrogen partial pressure at high temperature.

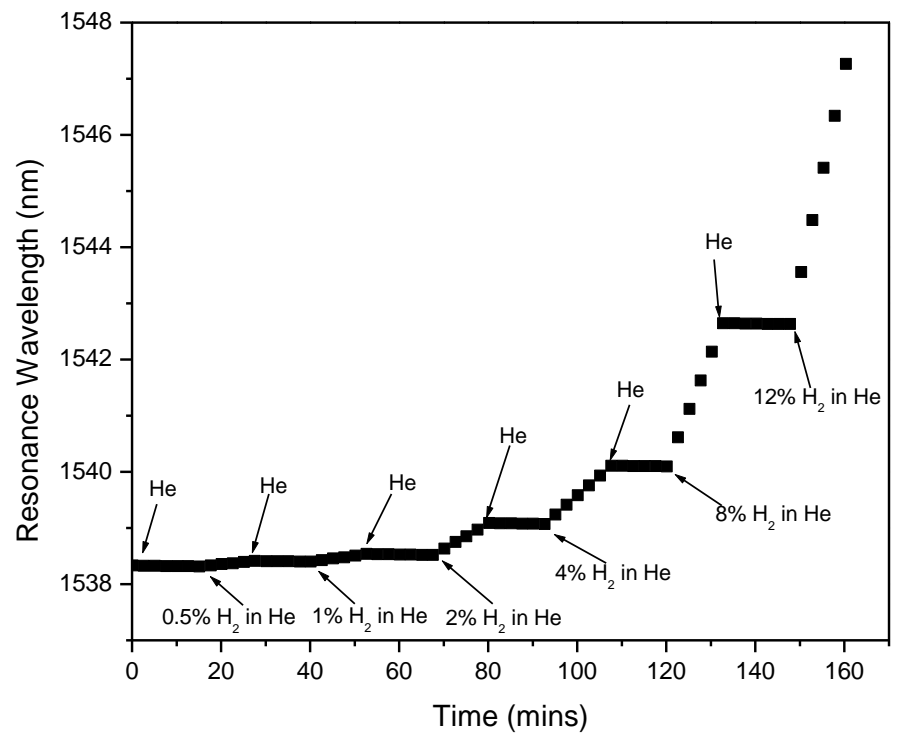

Figure 71 Resonance wavelength of SCTb-LPFGs as a function of time under various $\mathrm{H}_{2}$ partial pressure at $600{ }^{\circ} \mathrm{C}$.

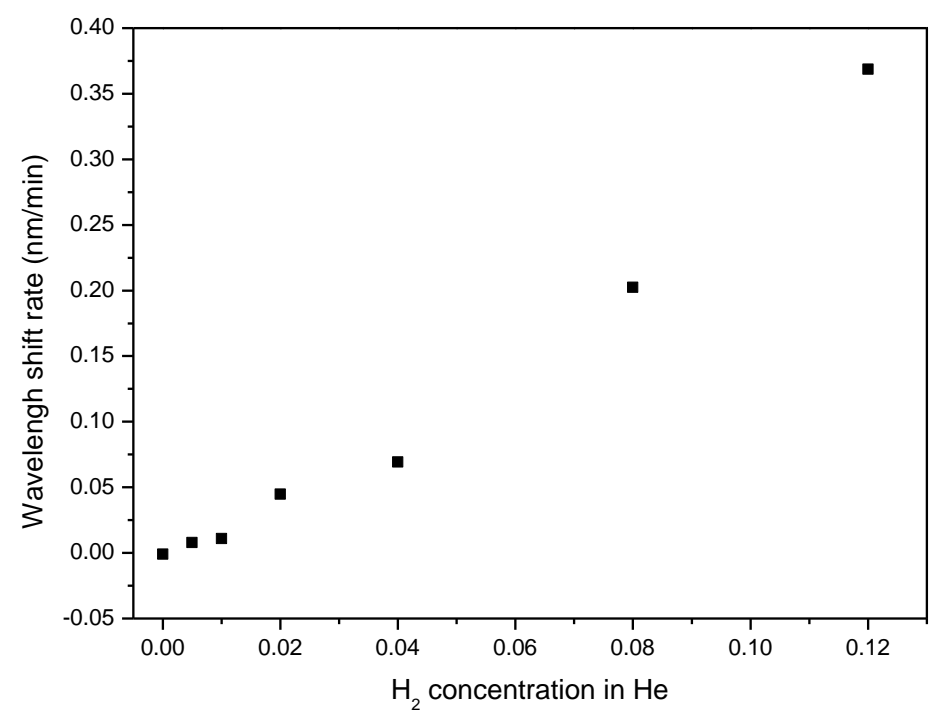

Figure 72 Resonance wavelength increase rate versus $\mathrm{H}_{2}$ partial pressure at $600{ }^{\circ} \mathrm{C}$ 


\subsubsection{Hydrogen Sensing Principle Investigation of SCTb}

\subsubsection{Refractive index study of $\mathrm{SCTb}$ with and without $\mathrm{H}_{2}$ treatment.}

A thin layer of $5 \% \mathrm{~Tb}$ doped $\mathrm{SrCeO}_{3}(\mathrm{SCTb})$ with a thickness of $150 \mathrm{~nm}$ was deposited onto a silicon waver by pulsed laser deposition. The refractive indices of SCTb before and after $\mathrm{H}_{2}$ treatment were quantified using spectroscopic ellipsometry (M-2000, J. A. Woollam) at room temperature. The relation between the refractive index and wavelength for the SCTb thin film prepared by pulsed laser deposition is shown in Figure 73. As seen in the figure, the refractive indices of the film gradually decrease from the near-UV region to infrared region, exhibiting the typical shape of a dispersion curve near an electronic interband transition. The same sample was then treated in $\mathrm{H}_{2}$ and $\mathrm{He}$ mixture containing $4 \% \mathrm{H}_{2}$ at $600^{\circ} \mathrm{C}$ for 5 hours followed by quenching down to room temperature in a short period of time without changing the gas environment to preserve the structure. As seen in Figure 73, the refractive indices of the treated sample decrease at all wavelength as compared to the fresh one.

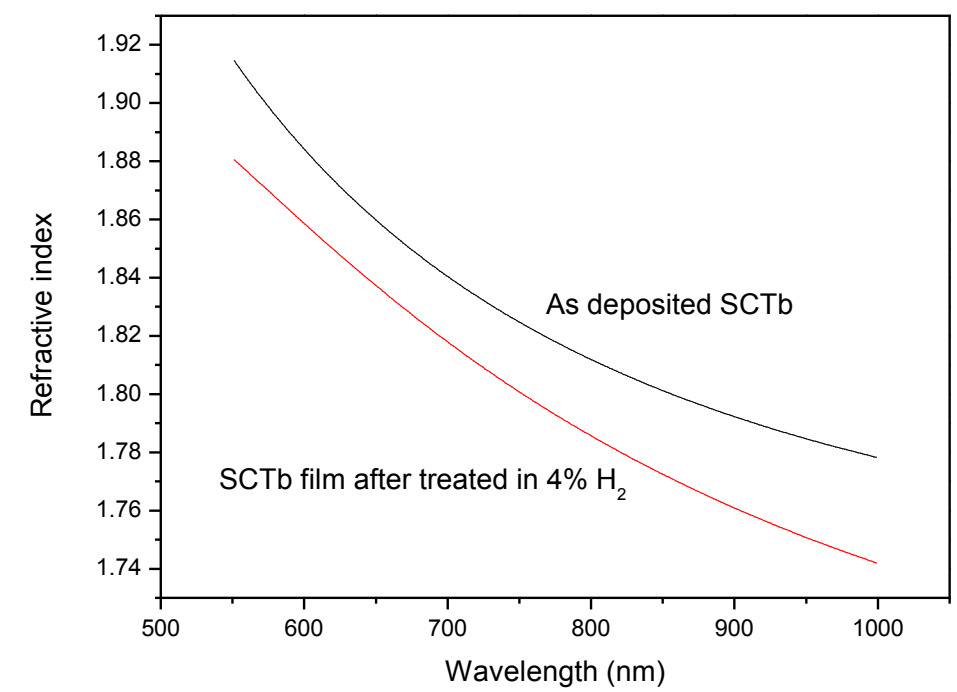

Figure 73 Refractive index of SCTb film with and without $\mathrm{H}_{2}$ treatment. 


\subsubsection{XRD pattern of SCTb powders with and without $\mathrm{H}_{2}$ treatment.}

It is well known that the refractive index of the materials is related to their crystallization defects and packing density. To characterize the crystal structure change of SCTb after treated in reducing environment, $\mathrm{X}$-ray diffraction (XRD) measurements with $\mathrm{Cu} \mathrm{K}_{\alpha}$ radiation were performed on SCTb powders. The powder was treated under same conditions as mentioned above. As seen in Figure 74, no secondary phase can be observed from the XRD pattern as compared to the as synthesized one.

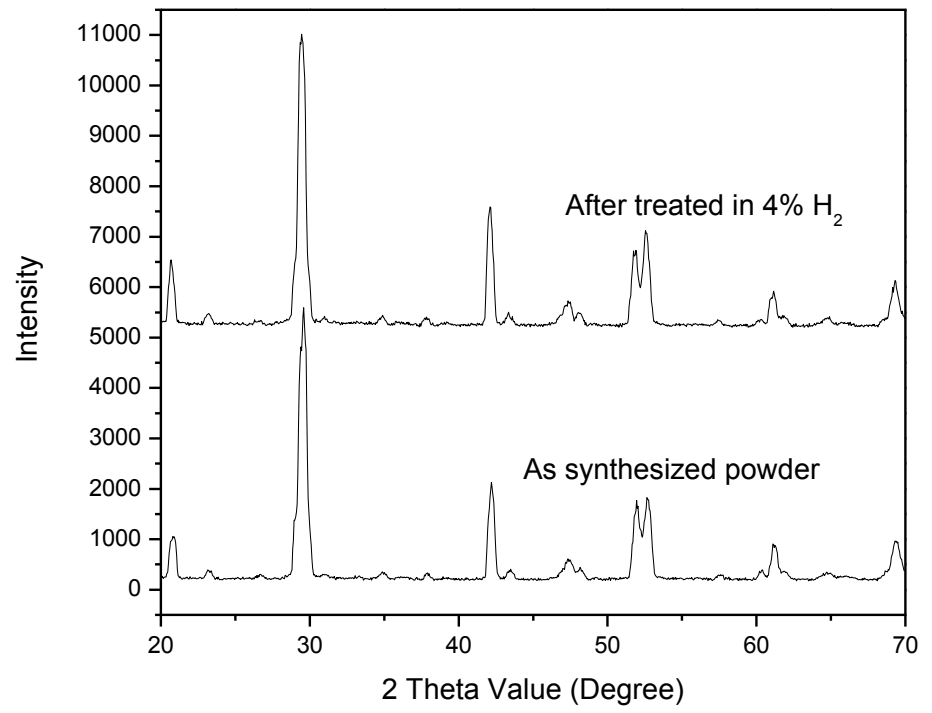

Figure 74 XRD pattern of as synthesized SCTb powder and one after treated in $4 \% \mathrm{H}_{2}$ at $600^{\circ} \mathrm{C}$ for 5 hours. 


\subsubsection{Surface Ce valence study by XPS}

To characterize the surface atom valence change, x-ray photoelectron spectroscopy (XPS) measurements were performed on SCTb powders. The depth of XPS analysis is estimated to be around $3 \mathrm{~nm}$. Figure 75 presents photoemission spectra for Ce before and after $\mathrm{H}_{2}$ treatment. For the as synthesized SCTb, the presence of the Ce $3 \mathrm{~d} 3 / 2$ and $\mathrm{Ce} 3 \mathrm{~d} 5 / 2$ are observed and the material is close match to photoemission spectra of $\mathrm{CeO}_{2}$. The peaks appear at lower EB indicating the relatively weaker $\mathrm{Ce}-\mathrm{O}$ bond in the perovskite structure as compared to the pure $\mathrm{CeO}_{2}$ fluoride structure. After treated in $\mathrm{H}_{2}$ under the same conditions as mentioned above, the relative intensity of the peaks decreases especially at 914 and $893 \mathrm{eV}$. This can be attributed to the reduction of $\mathrm{Ce}^{4+}$ into $\mathrm{Ce}^{3+}$.

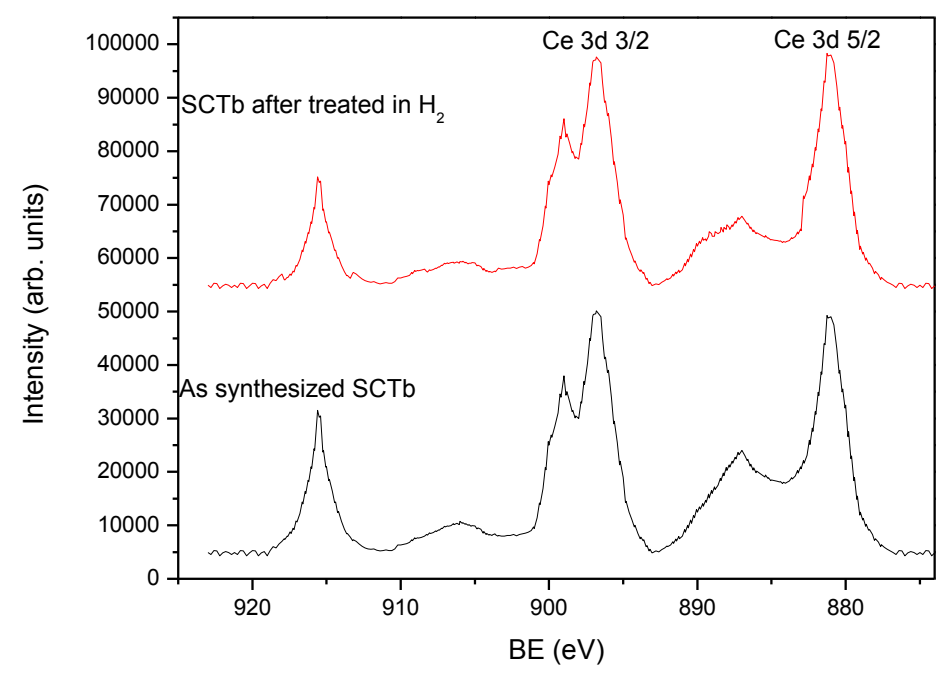

Figure 75 XPS spectra of as synthesized SCTb powder and after treated in $4 \% \mathrm{H}_{2}$ at $600^{\circ} \mathrm{C}$ for 5 hours 


\subsection{YSZ film Coating on Fiber}

Molten carbonate was selected as the $\mathrm{CO}_{2}$ sensing material because of its ability to serve as a media in which $\mathrm{CO}_{3}{ }^{2-}$ ion can transport. In order to form $\mathrm{CO}_{3}{ }^{2-}$ without the presence of $\mathrm{O}_{2}$, the molten carbonate needs to be attached to the material with high oxygen ionic conductivity which can supply $\mathrm{O}_{2}$. Molten carbonate also needs to be held by this material onto the optical fiber.

Among the material investigated before in our lab, YSZ seems to be more promising because of its high oxygen ion conductivity and chemical stability in harsh environment. Several approaches to coat YSZ film on the optical fiber have been investigated.

\subsubsection{YSZ thin film coating by sol-gel method}

YSZ sol containing YSZ particle size of 5nm was used to coat an YSZ thin layer on optical fiber. The SEM image is shown in Figure 76. As seen in the figure, the YSZ thin film contains very small pore size, which would inhibit the infiltration of the molten carbonate.
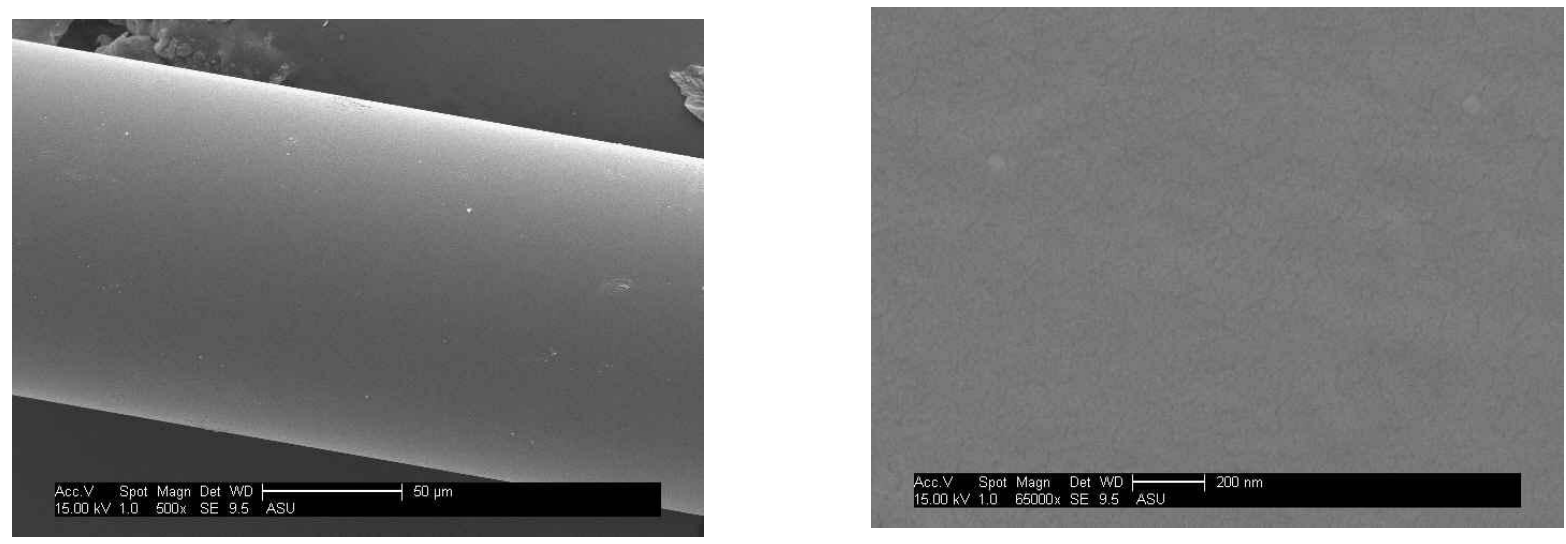

Figure 76 Optical fiber coated with YSZ thin film using YSZ sol 


\subsubsection{YSZ thin film coating by YSZ suspension}

As an alternative, a $10 \mathrm{wt} \%$ YSZ suspension with YSZ partial size of $200 \mathrm{~nm}$ was prepared by using isopropanol as the solvent. After multiple times coating, a film with much larger pore size was obtained (Figure 77). The film is able to hold the molten carbonate inside the pore due to the capillary force. The fiber will be tested at Missouri for $\mathrm{CO}_{2}$ sensing in Aug. 2008.
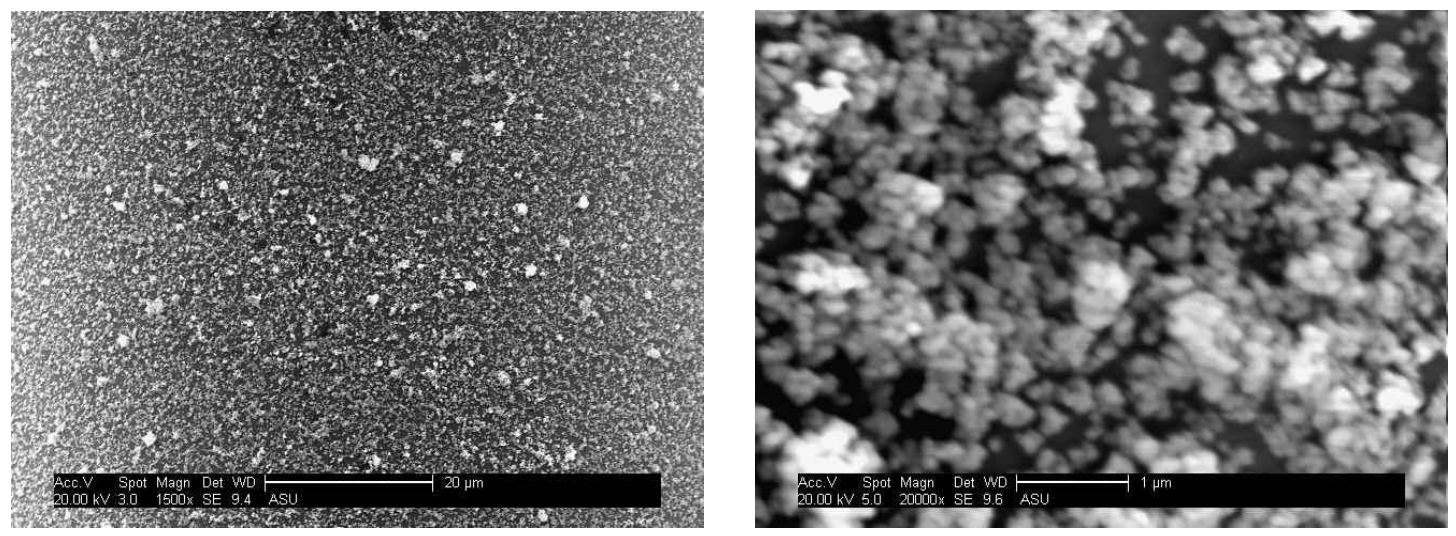

Figure 77 Optical fiber coated with YSZ thin film using YSZ suspension 


\subsection{Material selection and development for gas Sensing}

Various doped ceramic materials have been identified and engineered for selective gases detections at high temperatures. These materials have been characterized using various methods and the mechanisms of the sensing materials have been investigated.

\section{$\underline{2.8 .1 \mathrm{CO}_{2}} \underline{\text { Sensing : }}$}

For $\mathrm{CO}_{2}$ sensing, we investigated $\mathrm{BSCF}\left(\mathrm{Ba}_{0.5} \mathrm{Sr}_{0.5} \mathrm{Co}_{0.8} \mathrm{Fe}_{0.2} \mathrm{O}_{3-\delta}\right)$ type perovskite material and the dual phase, molten carbonate infiltrated in the pores of YSZ material. The molten carbonate phase was made of $42.5 \%$ lithium carbonate, $32.5 \%$ sodium carbonate and $25 \%$ potassium carbonate and a lanthanum cobaltite was the ceramic phase. We found that molten carbonate could selectively adsorb $\mathrm{CO}_{2}$ because $\mathrm{CO}_{2}$ could transport into the molten carbonate phase in the form of $\left[\mathrm{CO}_{3}=\right]$ with the help of electron and oxygen ion $(\mathrm{O}=)$ transport in the perovskite-type ceramic phase. To avoid the potential problem of phase changes during the carbonation reaction, we investigated the dual-phase, molten carbonate infiltrated in the pores of YSZ ceramic material. We found that the dual phase material helped avoid the formation of defect after several cycles of $\mathrm{CO}_{2}$ uptake.

We also found that BSCF $\left(\mathrm{Ba}_{0.5} \mathrm{Sr}_{0.5} \mathrm{Co}_{0.8} \mathrm{Fe}_{0.2} \mathrm{O}_{3-\delta}\right)$ could react with $\mathrm{CO}_{2}$ at high temperature to form $\mathrm{Ba}_{0.4} \mathrm{Sr}_{0.6} \mathrm{CO}_{3}$ with higher refractive index. Its perovskite type structure could be regained by recovering the material in Air. Therefore, BSCF can be used as an optical sensing material to monitor $\mathrm{CO}_{2}$ at high temperatures. 


\subsubsection{CO Sensing:}

For CO sensing, we synthesized and characterized the copper-doped zirconia (CDZ, $\mathrm{Cu}: \mathrm{Zr}=16: 84)$. XRD characterizations were performed to investigate the sensing mechanisms at various $\mathrm{CO}$ concentrations. We found that the CDZ nano materials were stable in the $200 \mathrm{ppm}$ $\mathrm{CO}$ in $\mathrm{N} 2 / \mathrm{CO} 2$ gas mixture at $550^{\circ} \mathrm{C}$. The sensor response was caused by the reversible Red-Ox reactions occurring when the atmosphere was switched between air and $\mathrm{CO} / \mathrm{CO}_{2} / \mathrm{N}_{2}$. However, when $\mathrm{CO}$ concentration is high $(>1 \%)$, complete reduction of $\mathrm{Cu}^{2+}$ to $\mathrm{Cu}^{0}$ occurs at high temperature. The metal $\mathrm{Cu}$ segregates from the $\mathrm{CDZ}$ solid solution to the crystal boundaries as indicated by the appearance of $\mathrm{Cu}$ peaks. This caused the irreversibility of the $\mathrm{CDZ}$ based $\mathrm{CO}$ sensor in high concentrations.

\section{$\underline{2.8 .3 \mathrm{H}_{2}} \underline{\text { S Sensing: }}$}

For $\mathrm{H}_{2} \mathrm{~S}$ sensing, we mainly evaluated the tin oxide which is common used in current $\mathrm{H}_{2} \mathrm{~S}$ sensors. The $\mathrm{SnO}_{2}$ nano-crystalline thin films were successfully synthesized on the LPFG surface by nanoparticle incorporated polymeric precursors and direct sol-gel methods. The sensors were demonstrated to respond to $\mathrm{H}_{2} \mathrm{~S}$ in $\mathrm{N}_{2}$. The Pd-LPFGs sensor was also tested in the temperature range of $30-250{ }^{\circ} \mathrm{C}$ under various hydrogen concentrations. After hydrogen was introduced into the system, the shift of the resonance wavelength towards the lower wavelength was observed. In our work, the shift of resonance wavelength is found to be $+35 \mathrm{pm} /{ }^{\circ} \mathrm{C}$ in He. When tested in the temperature range of $30-200^{\circ} \mathrm{C}$, the resonance wavelength of the Pd-LPFGs shifted towards the lower wavelength in $8 \% \mathrm{H} 2 / \mathrm{He}$ as compared to $\mathrm{He}$. At $250^{\circ} \mathrm{C}$, the amount of hydrogen dissolved into Pd is too small to cause any observable and reproducible wavelength shift in the hydrogen concentration range studied in this work (0-16\%). 
It was observed that the resonance wavelength decreases as the hydrogen concentration increases at all temperatures. At $30^{\circ} \mathrm{C}$, there is a sharp decrease in the resonance wavelength when hydrogen concentration increases from $1 \%$ to $2 \%$. This is due to a jump in hydrogen concentration (and lattice parameter) in $\mathrm{Pd}$ as a result of $\beta$ to $\alpha$ phase transition. These changes cause drastic changes in refractive index, resulting in the pronounced shift of the resonance wavelength. Further increasing the hydrogen concentration from $2 \%$ to $16 \%$ leads to a much less significant wavelength shift because $\mathrm{PdH}$ is in the $\alpha$ phase, in which hydrogen concentration increases continuously with the gas phase hydrogen concentration. The extent of resonance wavelength shift highly depends on the temperature. As explained in the principle, the hydrogen absorption capacity of Pd decreases significantly as the temperature increases, causing a less change in the refractive index of Pd at higher temperatures. It should be noted that at $200^{\circ} \mathrm{C} \mathrm{a}$ shift of $-11 \mathrm{pm}$ in the resonance wavelength can be observed when exposed to $4 \% \mathrm{H} 2$ even though the ratio between $\mathrm{H}$ and $\mathrm{Pd}$ is smaller than 0.01 in $\mathrm{PdH}_{\mathrm{x}}$. This indicates the high resolution of this technique for hydrogen sensing up to $200^{\circ} \mathrm{C}$. However, the change is smaller than the response induced by $-1^{\circ} \mathrm{C}$ temperature variation. Therefore, it is necessary to take into account the gas temperature variations in the operation of such a sensor.

The response and recovery time of the sensor is mainly determined by the surface reaction and hydrogen diffusion process in Pd. The transport of hydrogen through the Pd layer involves three steps. Once the Pd thin layer is exposed to hydrogen, hydrogen is dissociated into two hydrogen atoms or forms protons and then the atoms diffuse into the bulk metal. In the bulk, there is a net transport of hydrogen atoms driven by the hydrogen concentration gradient. The hydrogen atoms recombine and form hydrogen molecules again during the desorption process. The recovery time is longer than the response time at lower temperatures. This indicates a slower 
hydrogen desorption rate corresponding the phase change from $\beta$ to $\alpha$. It is attributed to the macroscopic energy barrier between the transforming phases arising from the coherent strains, which opposes both the forward and reverse iso-structural hydriding transformations. At elevated temperatures, the recovery time is much faster for the sensor without going through the phase change.

The use of Pd-coated LPFGs as a hydrogen sensor in the temperature range of $30-200^{\circ} \mathrm{C}$ is demonstrated in this work. The exposure of the sensor to hydrogen leads the attenuation band of the transmission spectrum of LPFGs to shift towards the lower wavelength at all the temperatures. The resonance wavelength decreases as the hydrogen concentration increases in the range from $0 \%$ to $16 \%$. There is a significant wavelength shift of the sensor when the Pd film goes through the $\alpha$ to $\beta$ phase change. The sensor has short response time with relatively long recovery time at low temperatures. Multiple cycle test confirms the stability of the sensor for hydrogen detection. The results show feasibility of using LPFGs, when coated with an appropriate sensing material, offering the potential for sensing hydrogen at high temperatures in the IGCC streams. Future work will focus on exploration of other sensing materials with improved sensitivity and chemical stability for detection of hydrogen and other gases in IGCC streams. 


\subsection{Investigation of Nano-crystalline Copper-Doped Zirconia Thin Films for Optical Sensing of Carbon Monoxide at High Temperature}

Recently, the long period fiber grating (LPFG) devices have been considered with great potential for constructing refractive index gas sensors by coating with gas-sensitive films. The LPFG is a fiber device containing a refractive index modulation in the core of the fiber that can be created by laser irradiation. When light propagates through the LPFG, a series wavelength (i.e. resonant wavelength, $\lambda_{R}$ ) are coupled from core mode to cladding modes. The $\lambda_{\mathrm{R}}$ of a specific LPFG is determined by the difference between the effective indices of core mode $\left(n_{\text {eff,co }}\right)$ and cladding mode $\left(n_{\text {eff,clad }}\right)$ and the grating period $(\Lambda)$ typically in a range of $100-1000 \mu \mathrm{m}$. The $\lambda_{R}$ of the LPFG shifts when the refractive index of the surrounding medium changes because the effective indices of the cladding modes, $n_{\text {eff,clad }}$, depend on the cladding index and the index of the surrounding. Thus, LPFG-based sensors can be built by coating a material whose refractive index varies sensitively when contacting specific chemicals.

We demonstrated a proton-conducting perovskite-type $\operatorname{Sr}\left(\mathrm{Ce}_{0.8} \mathrm{Zr}_{0.1}\right) \mathrm{Y}_{0.1} \mathrm{O}_{2.95}$ (SCZY) thin film coated LPFG for hydrogen sensing at around $500^{\circ} \mathrm{C}$. At high temperature, the types and populations of the ionic defects in the SCZY solid will change when the surrounding hydrogen partial pressure varies. The change in defect states affects the refractive index of the SCZY film that makes the SCZY-LPFG capable of detecting hydrogen at high temperature by monitoring its resonant wavelength shift $\left(\Delta \lambda_{R}\right)$.

Nano - crystalline copper-doped zirconia (CDZ) thin films were synthesized and investigated as a potential high temperature CO-sensing material for coating the LPFG. Many doped zirconia materials are known as good redox catalysts that exhibit reversible changes of material properties 
under reducing-oxidizing cycles at high temperature. The hypothesis of this research is that the reversible $\mathrm{Cu}^{2+} \Leftrightarrow \mathrm{Cu}^{+}$redox activities in the crystalline structure will change the $\mathrm{CDZ}$ refractive index to allow for optical sensing of $\mathrm{CO}$ at high temperatures. 


\subsection{Synthesis and Characterization of CDZ film}

Chemicals used in this study include zirconyl chloride hydrate $\left(\mathrm{ZrOCl}_{2}-8 \mathrm{H}_{2} \mathrm{O}, 99.99 \%\right.$, Aldrich), glycine (99+\%, Aldrich), ethylene glycol (99+\%, Aldrich), and $\mathrm{CuCl}_{2}-8 \mathrm{H}_{2} \mathrm{O}(99.99 \%$, Aldrich). The polymeric precursor, i.e. $\left(\mathrm{Zr}^{4+}, \mathrm{Cu}^{2+}\right)$-chelated poly ethyl glycol, was synthesized by polymerization of an aqueous solution containing ethylene glycol, zirconyl chloride, copper chloride, and glycine. Glycine was used as a $\mathrm{pH}$ control agent $(6<\mathrm{pH}<7)$ to inhibit the formation of precipitates during polymerization upon heating. The polymeric precursor synthesis procedure was similar to that reported in the literature and used in our previous work. The $\mathrm{Cu}: \mathrm{Zr}$ atomic ratio of the precursor was 16:84 which is the same as the ratio in final CDZ oxides. The molar ratio of ethyl glycol to the total amount of metal ions was 1:1. After about 60 minutes of vigorous stirring, the starting solution is of green color free of precipitates. The solution was contained in a $125-\mathrm{ml}$ glass flask with open mouth and placed in an oven at $80^{\circ} \mathrm{C}$ to polymerize for a certain period of time, typically $144 \mathrm{~h}$. The polymerized solution was cooled and placed statically at room temperature for $48 \mathrm{~h}$ before coating thin films. The polymerization duration and room temperature aging time were determined through a series of trials and found to give a precursor viscosity suitable for coating uniform precursor films that can result in crack-free solid oxide thin films. The thus obtained precursor remains good for coating for one week when stored at room temperature in a closed flask. The polymeric precursor was examined by FTIR to confirm the metal-chelated polymer structure.

The LPFG used in this work was the same as those used in our previous studies. The LPFG was made from Corning SMF-28 fiber (refractive index 1.4682, $\varphi 125-\mu \mathrm{m}$ pure silica cladding and $\varphi 9-\mu \mathrm{m}$ Ge-doped silica core) by $\mathrm{CO}_{2}$-laser irradiation with a grating period $(\Lambda)$ of $520 \mu \mathrm{m}$ 
and a total grating length of $\sim 5 \mathrm{~cm}$. The fifth coupling wavelength is specifically used for $\lambda_{R}$ monitored in a near IR wavelength range of $1510-1640 \mathrm{~nm}$. The LPFG has good long-term thermal stability at $550^{\circ} \mathrm{C}$ with a $\lambda_{R}$ thermal drift rate of about $-0.0095 \mathrm{~nm} / \mathrm{h}$ and a linear temperature dependence of $\sim 0.1 \mathrm{~nm} /{ }^{\circ} \mathrm{C}$ for $\lambda_{\mathrm{R}}$ in a wide temperature range from 100 to $800^{\circ} \mathrm{C}$. The detailed LPFG fabrication conditions and procedure have been reported elsewhere.

The polymeric precursor film coating on the LPFG fiber was accomplished by pulling a drop of the polymer solution held by the tip of a small brush along the LPFG fiber segment suspended horizontally in a tubular furnace. The drop sliding speed is about $1 \mathrm{~cm} / \mathrm{s}$ and this brush coating process was repeated three times. The precursor coated fiber was then dried at $100^{\circ} \mathrm{C}$ for $5 \mathrm{~min}$ or cured at room temperature for a few hours and fired at $650^{\circ} \mathrm{C}$ for $2 \mathrm{~h}$ using a rapid heating rate of $\sim 50^{\circ} \mathrm{C} / \mathrm{min}$. The CDZ film coated LPFG used for gas sensing test were obtained by four cycles of the polymer coating and firing process. The four-time coated CDZ-LPFG was treated by a final step of annealing at $750^{\circ} \mathrm{C}$ for $30 \mathrm{~min}$ to stabilize the $\mathrm{CDZ}$ nano-grains and enhance the thermal stability of the LPFG for application at a lower temperatures of $500-600^{\circ} \mathrm{C}$. It should be noted that the present work focuses primarily on the chemical and microstructural properties of the CDZ sensing materials but does not involve optimization of the coating times or the film thickness which is known to affect the sensitivity of the film-coated LPFG sensors.

For materials characterization and properties studies, CDZ thin films on silicon wafers and particle samples were also prepared from the polymeric precursors under the thermal treatment procedure identical to that of the CDZ-LPFG fabrication process. The CDZ films on silicon wafers was prepared by spin-coating at a speed of 2,000 rpm followed by drying on a hot plate at $100^{\circ} \mathrm{C}$ and $300^{\circ} \mathrm{C}$, respectively, and firing at $650^{\circ} \mathrm{C}$. The $\mathrm{CDZ}$ powder samples were prepared by firing a 1 2-mm thick layer of precursor in a flat bottom crucible at $650^{\circ} \mathrm{C}$. The $\mathrm{CDZ}$ crystal 
phase was identified by X-ray diffraction (XRD). The CDZ film thicknesses on the fiber and Si wafer were determined by scanning electron microscope (SEM) and the grains sizes of the oxides were measured by high resolution transmission electron microscope (HRTEM). An ellipsometer was used to measure the refractive index of the CDZ films in a near IR wavelength range of 1510 to $1640 \mathrm{~nm}$, which was the same wavelength range used for optical sensing test for the CDZ-LPFG. 


\section{Sensor Tests and Technology Demonstration}

\subsection{Instrumentation and sensor testing of gas sensors}

The performance of the integrated sensors were tested and verified in laboratory simulated gas mixtures at high temperatures. Although all the developed sensors have been tested, our primary efforts have been focused on evaluating the $\mathrm{H}_{2}$ sensors. The sensors have been tested in single-component as well as multi-component high temperature environments, showing high sensitivity ( ppm), large dynamic range (ppm to $80 \%$ for hydrogen sensing), long lifetime (>60 days), and high operating temperature $\left(>500^{\circ} \mathrm{C}\right)$. Our encouraging laboratory test results suggested that the $\mathrm{H}_{2}$ sensors are now ready for real world tests.

Instrumentation of the sensor system was successfully developed and implemented in two locations (University of Cincinnati and Missouri University of Science and Technology) to support the sensor development, test and evaluations. Signal processing algorithms have been developed based on mathematic and physical models of the sensor device.

\section{$\underline{\text { 3.1.1 H}} \underline{2}_{2}$ sensors}

The SCZY-LPFG sensors were first tested in $\mathrm{H}_{2} / \mathrm{N}_{2}$ and then CO/ $\mathrm{N}_{2}$ binary gas mixtures, respectively. The measurements were conducted at $500^{\circ} \mathrm{C}$ and 1 atm total pressure. The SCZYLPFG was able to respond sensitively to the change of $\mathrm{H}_{2}$ concentration in a range of 0 to $80 \%$, indicating its ability to quantify bulk $\mathrm{H}_{2}$ concentrations.

The sensor's responses to $\mathrm{CO}, \mathrm{CO}_{2}, \mathrm{H}_{2} \mathrm{~S}$, and $\mathrm{H}_{2} \mathrm{O}$ were tested in binary mixtures with $\mathrm{N}_{2}$ to evaluate the potential interferences of these gases to the quantitative detection of $\mathrm{H}_{2}$. The results 
indicated that sensor's responses to these gases were insignificant as compared to the response to $\mathrm{H}_{2}$.

The SCZY-LPFG sensors were also tested in two multicomponent mixtures (as shown in Figure 78), i.e. (1) $\mathrm{H}_{2}$ in $\mathrm{N}_{2}+10 \% \mathrm{CO}_{2}+30 \% \mathrm{CO}+2.8 \% \mathrm{H}_{2} \mathrm{O}$, and (2) $\mathrm{H}_{2}$ in $\mathrm{N}_{2}+20 \% \mathrm{CO}_{2}+20 \% \mathrm{CO}+2.8 \% \mathrm{H}_{2} \mathrm{O}$, where the concentrations of coexisting gases were on the basis of the amount excluding $\mathrm{H}_{2}$. The sensor responses to $\mathrm{H}_{2}$ concentration variations in the multicomponent mixture were in excellent agreement with that in the $\mathrm{H}_{2} / \mathrm{N}_{2}$ binary mixture. These results indicate that the SCZY-LPFG sensors have a great potential for measurement of bulk $\mathrm{H}_{2}$ in syngas with minimal interference from the reducing $\mathrm{CO}$ content.

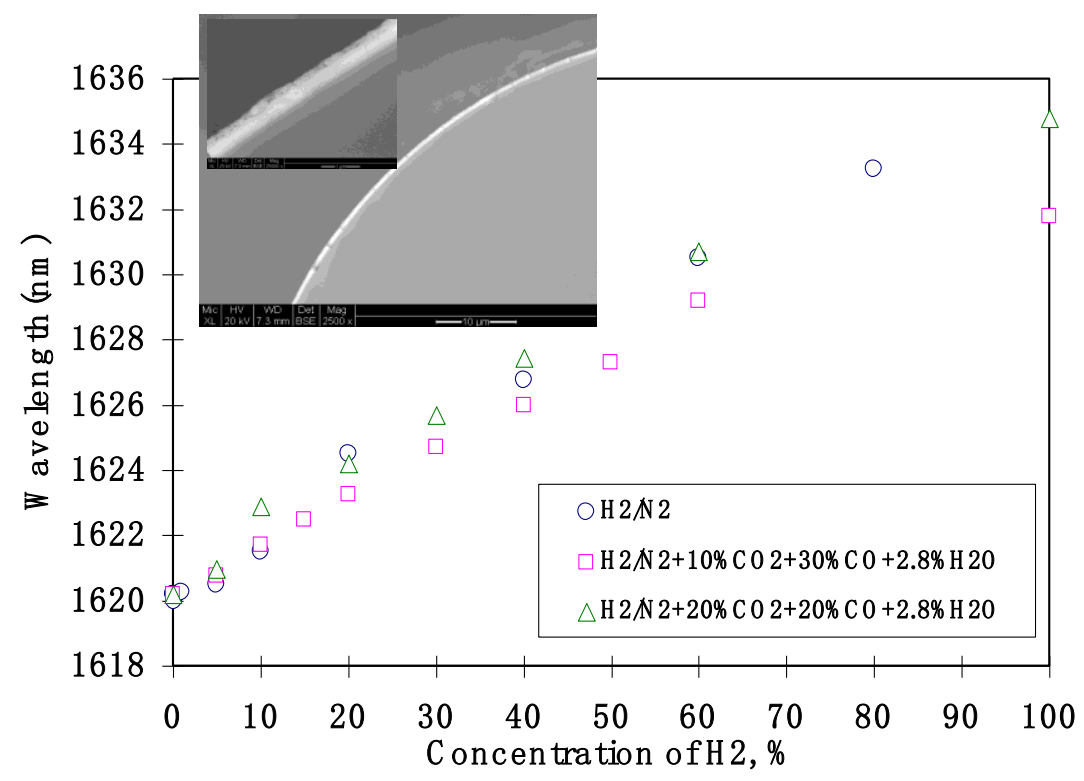

Figure $78 \mathrm{SCZY}$ coated LPFG in response to $\mathrm{H}_{2}$ concentration variations 


\subsubsection{CO sensors}

The $\mathrm{CDZ}\left(\mathrm{ZrO}_{2} / \mathrm{CuO}\right)-\mathrm{LPFG}$ sensors were tested for $\mathrm{CO}$ detection in $\mathrm{CO}_{2}$ at $550^{\circ} \mathrm{C}$ and atmospheric pressure for more than $250 \mathrm{hr}$. The sensor was capable of detecting $\mathrm{CO}$ concentration in a range from 0 to $\sim 1,000 \mathrm{ppm}(0.1 \%)$ with excellent sensitivity. However, when the $\mathrm{CO}$ concentration was $>1 \%$, the sensor response became irreversible. Testing continues to be performed on the sensor.

\section{$\underline{\text { 3.1.3 } \mathrm{CO}_{2}} \underline{\text { sensors }}$}

BSCF $\left(\mathrm{Ba}_{0.5} \mathrm{Sr}_{0.5} \mathrm{Co}_{0.8} \mathrm{Fe}_{0.2} \mathrm{O}_{3-\delta}\right)$-LPFG sensors have been tested for $\mathrm{CO}_{2}$ sensing in $\mathrm{CO}_{2} /$ Air mixture at temperatures higher than $500^{\circ} \mathrm{C}$. The sensor responded reversibly to the variations of $\mathrm{CO}_{2}$ concentrations. The response time was on the order of a few minutes.

\section{$\underline{3.1 .4 \mathrm{H}_{2}} \underline{\mathrm{S} \text { sensors }}$}

The $\mathrm{SnO}_{2}$ nano-crystalline film coated LPFG sensors were tested for $\mathrm{H}_{2} \mathrm{~S} / \mathrm{N}_{2}$ binary mixtures at $550^{\circ} \mathrm{C}$. The sensors responded to $\mathrm{H}_{2} \mathrm{~S}$ with a red shift in resonant wavelength. Although the shift of resonant wavelength appeared to have quantitative relationship with the $\mathrm{H}_{2} \mathrm{~S}$ concentration at ppm levels, the response was found to be irreversible. The $\mathrm{SnO}_{2}$ - $\mathrm{LPFG}$ sensors are therefore not suitable for monitoring $\mathrm{H}_{2} \mathrm{~S}$ concentration fluctuation but maybe useful in "ONOFF" detections.

So far, the proposed research tasks have been performed according to the schedule and all the major milestones of the project have been accomplished successfully. Our fundamental research has demonstrated the feasibility of the new type of fiber optic gas sensors in laboratory tests. 


\subsection{SCZY-LPFG Sensor fabrication and test}

For the planned test of the high temperature $\mathrm{H}_{2}$ sensor in actual gasification systems, we prepared total six SCZY-LPFG sensors. The sensor preparation procedure was the same as described in previous reports to DOE and that in our publications. Three of the sensors were found to have excellent quantitative dependence of sensor output on the $\mathrm{H} 2$ concentration at 500 ${ }^{\circ} \mathrm{C}$ and high selectivity for $\mathrm{H}_{2}$ over other gases, e.g. $\mathrm{CO}, \mathrm{CO}_{2}, \mathrm{H}_{2} \mathrm{O}$, and $\mathrm{CH}_{4}$. These sensors are now installed in $1 / 4$ " stainless steel tube ready for retest in lab condition. The sensors will be shipped to MST (Xiao's group) for packaging for the test on gasifier.

\subsubsection{Synthesis of SCTb nanofilm on the LPFG}

Nanocrystalline $\mathrm{SrCe}_{0.95} \mathrm{~Tb}_{0.05} \mathrm{O}_{3-\mathrm{a}}(\mathrm{SCTb})$ thin films were synthesized on the LPFG cylindrical surface by the polymeric precursor route. The fabrication process mainly includes three steps: (1) synthesis of the metal-chelated polymeric precursor by polymerizinga solution (under acidic condition) containing $\mathrm{Sr}^{2+}, \mathrm{Ce}^{4+}, \mathrm{Tb}^{3+}$, anions, $\mathrm{H}_{2} \mathrm{O}$, ethylene glycol and glycine. The composition of the starting solution is consistent with the stoichiometric ratio of metal ions in the oxide. The polymerization is conducted at $80^{\circ} \mathrm{C}$ for $72-144$ hours followed by $24-120$ hours of aging period at room temperature. The film was obtained by five-time coating which resulted in a thickness of $\sim 400 \mathrm{~nm}$ after firing at $650^{\circ} \mathrm{C}$. The SEM pictures of a single-time coated polymeric precursor film and the SCTb-LPFG are shown in Figure 79 and Figure 80 respectively. 


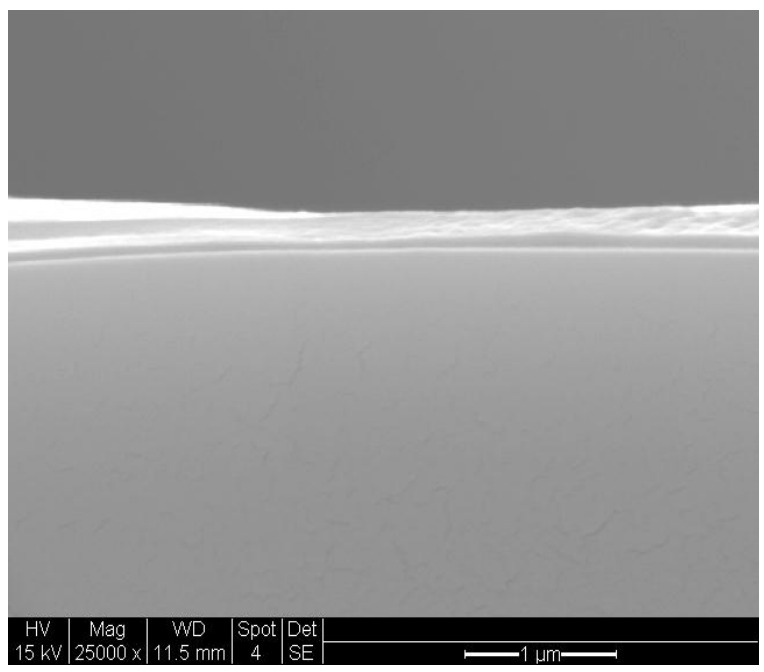

Figure 79 SEM images of single-time coated SCTb-polymeric thin film: (a) pre-fire; (b) post-fire.

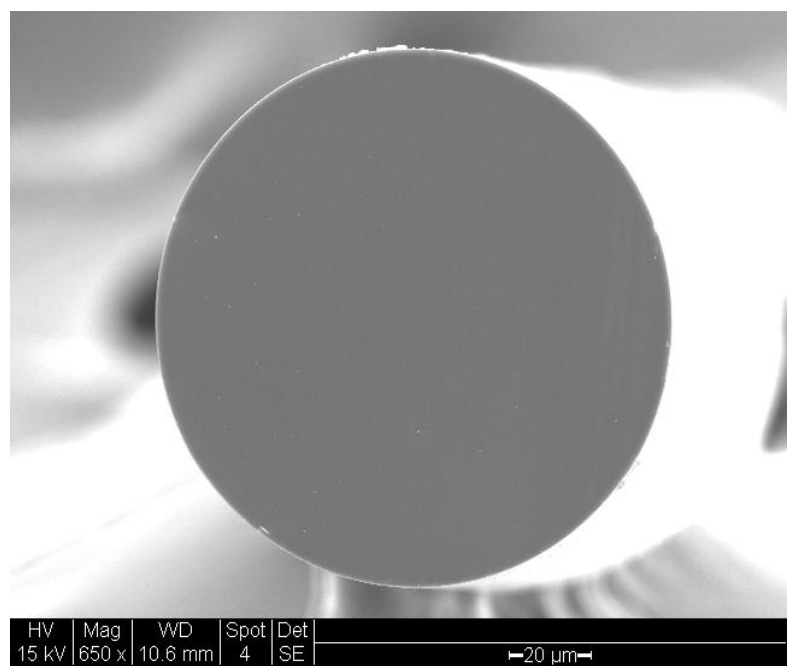

(a) 


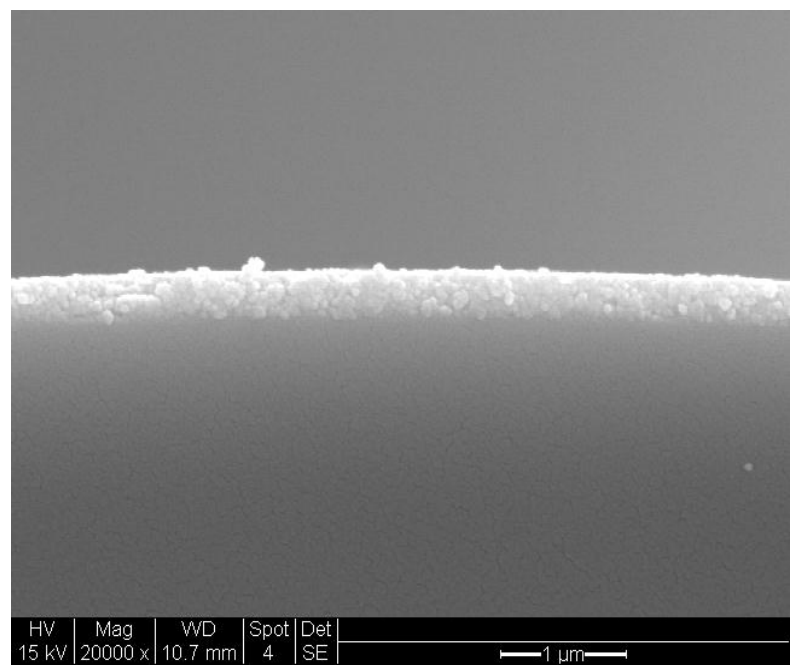

(b)

Figure 80 SEM images of the 5-time coated SCTb-LPFG sensor showing 400nm SCTb nano crystalline thin film on the LPFG.

\subsubsection{SCTb-LPFG sensor response to $\mathrm{H}_{2}, \mathrm{CO}_{2}$, and $\mathrm{CO}$}

The SCTb-LPFG sensor was tested in $\mathrm{H}_{2} / \mathrm{N}_{2}, \mathrm{CO}_{2} / \mathrm{N}_{2}$ and $\mathrm{CO} / \mathrm{N}_{2}$ binary gas mixtures, respectively. The measurements were conducted at $500^{\circ} \mathrm{C}$ and 1 atm total pressure. Figure 81 shows the results of the measurement. The SCTb-LPFG responded with red shifts of $\lambda_{R}$ (Figure 81 a) as the $\mathrm{H}_{2}$ concentration increased from 0 to $100 \%$ (Figure 81 (b)) indicating its ability to quantify bulk $\mathrm{H}_{2}$ concentrations. The SCTb-LPFG exhibited very small response to the variation of $\mathrm{CO}$ and $\mathrm{CO}_{2}$ concentration in the full range from 0 to $100 \%$ (Figure 81(b)). The SCTb-LPFG sensor responses to $\mathrm{H}_{2} / \mathrm{N}_{2}, \mathrm{CO}_{2}$ and $\mathrm{CO} / \mathrm{N}_{2}$ binary gas mixtures were very similar to those of the SCZY-LPFG sensor. In a $\mathrm{H}_{2}$ concentration range of $5-40 \%$, the sensor's average selectivity for $\mathrm{H}_{2}$ over other gases was $\sim 11.5$. 


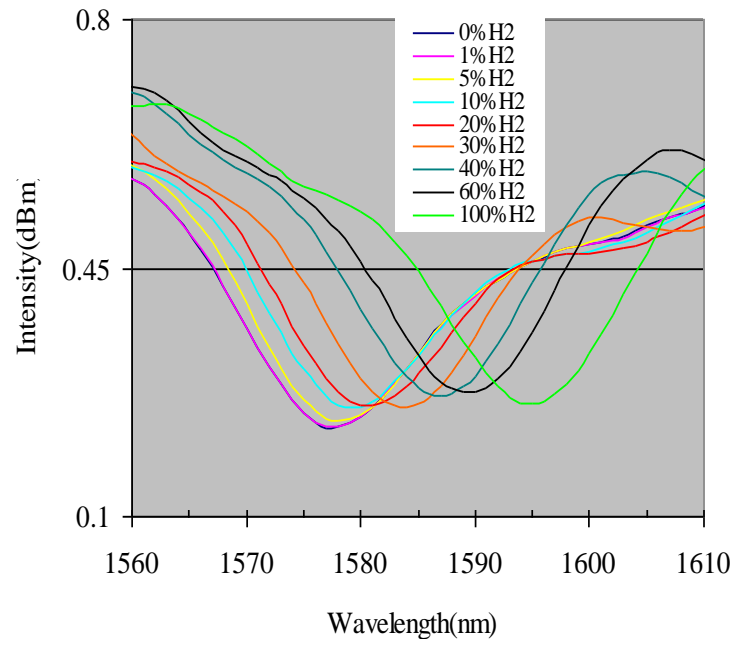

(a)

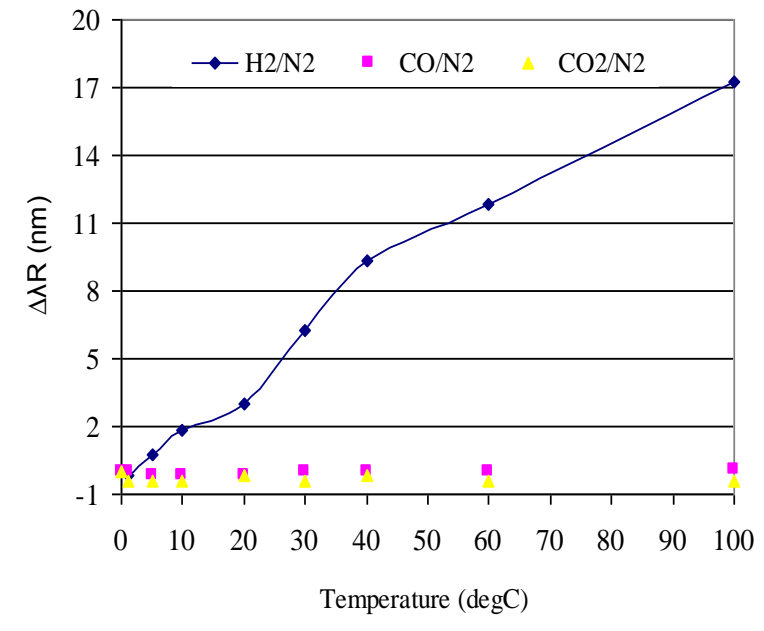

(b)

Figure 81 Results of SCTb-LPFG sensor tests in $\mathrm{H} 2 / \mathrm{N} 2, \mathrm{CO} / \mathrm{N} 2$ and CO2/N2 binary gas mixtures at $500 \mathrm{oC}$ and $1 \mathrm{~atm}$ : (a) Stabilized transmission spectra in $\mathrm{H} 2 / \mathrm{N} 2$ with various $\mathrm{H} 2$ concentrations; (b) $\lambda \mathrm{R}$ shift $(\Delta \lambda \mathrm{R})$ as a function of $\mathrm{H} 2, \mathrm{CO}$ and $\mathrm{CO}_{2}$ concentrations.

\subsubsection{SCZY, SCTb, and SCTm Nano film coated LPFG sensor fabrication}

Five $\operatorname{Sr}\left(\mathrm{Ce}_{0.8} \mathrm{Zr}_{0.1}\right) \mathrm{Y}_{0.1} \mathrm{O}_{2.95}(\mathrm{SCZY})$ coated LPFG sensors have been fabricated, tested on bench top system and sent to MST for packaging; $\mathrm{SrCe}_{0.95} \mathrm{~Tb}_{0.05} \mathrm{O}_{3-\mathrm{a}}(\mathrm{SCTb})$ coated LPFG sensors have been fabricated and tested in the lab; and $\mathrm{SrCe}_{0.95} \mathrm{Tm}_{0.05} \mathrm{O}_{3-\mathrm{a}}(\mathrm{SCTm})$ coated LPFG sensors have been fabricated but not tested yet.

The $\operatorname{Sr}\left(\mathrm{Ce}_{0.8} \mathrm{Zr}_{0.1}\right) \mathrm{Y}_{0.1} \mathrm{O}_{2.95}(\mathrm{SCZY}), \mathrm{SrCe}_{0.95} \mathrm{~Tb}_{0.05} \mathrm{O}_{3-\mathrm{a}}(\mathrm{SCTb})$, and $\mathrm{SrCe}_{0.95} \mathrm{Tm}_{0.05} \mathrm{O}_{3-\mathrm{a}}$ (SCTm) nano films have been coated on LPFGs using the polymeric precursor synthesis route. The SCZY-LPFG and SCTb-LPFG have been previously reported and the SCTm-LPFG sensors are synthesized for the first time by slightly modified fabrication procedure. The $\mathrm{SrCe}_{0.95} \mathrm{Tm}_{0.05} \mathrm{O}_{3-\mathrm{a}}(\mathrm{SCTb})$ nano crystalline thin film fabrication process includes three steps similar to those used in SCZY films synthesis: (1) synthesis of the metal-chelated polymeric precursor by polymerizing solution (under acidic condition) containing $\mathrm{Sr}^{2+}, \mathrm{Ce}^{4+}, \mathrm{Tm}^{3+}$, anions, 
$\mathrm{H}_{2} \mathrm{O}$, ethylene glycol and glycine. The composition of the starting solution is consistent with the stoichiometric ratio of metal ions in the oxide. The polymerization is conducted at $80^{\circ} \mathrm{C}$ for $100-$ 150 hours followed by one day of aging period at room temperature. The film was obtained by five-time coating which resulted in a thickness of $\sim 400 \mathrm{~nm}$ after firing at $650^{\circ} \mathrm{C}$.

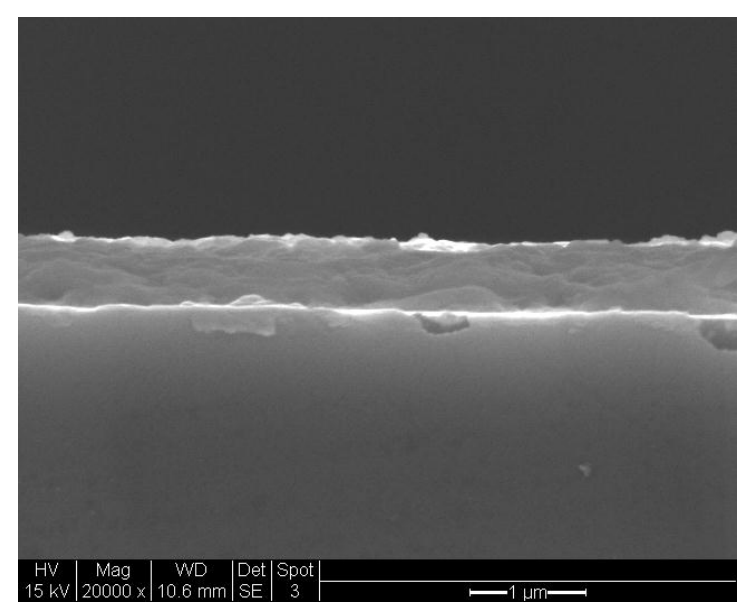

(a) $\mathrm{SCTb}$

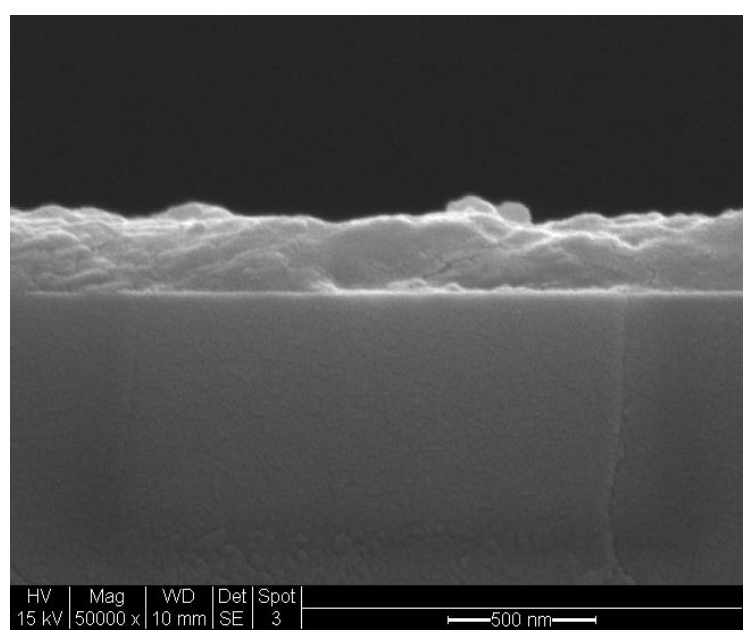

(b) SCTm 


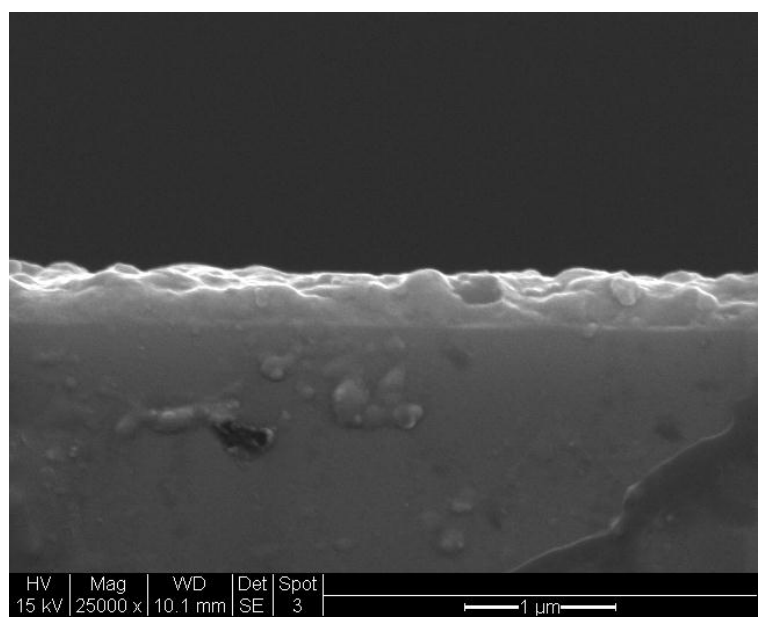

(c) SCZY

Figure 82 SEM images of pervoskite thin films on optical substrates: (a) SCTb thin film; (b) SCTm thin film; (c) SCZY thin film.

To ensure the preservation of the optical function of the SCZY- LPFG device, the transmission spectrum was measured in different stages of the film synthesis. The final SCZYLPFG exhibits a strong resonant peak which is necessary for quantitative measurement of $\Delta \lambda_{R}$. The stability of the SCZY- LPFG at $500^{\circ} \mathrm{C}$ in terms of $\lambda_{\mathrm{R}}$ thermal drift was $\sim-6.5 \times 10-3 \mathrm{~nm} / \mathrm{h}$ as measured over a period of $240 \mathrm{~h}$. The experimental values of $\lambda_{\mathrm{R}}$ presented later in this technical note were compensated with this thermal drifting rate. The TEM examination of the unsupported SCZY samples showed that the SCZY grain size increased from 5 to $10 \mathrm{~nm}$ after firing at $550^{\circ} \mathrm{C}$ to $10-15 \mathrm{~nm}$ after firing at $700{ }^{\circ} \mathrm{C}$. The grain size of the SCZY fired at $700^{\circ} \mathrm{C}$ showed no appreciable change after further calcination at $500{ }^{\circ} \mathrm{C}$ for more than $100 \mathrm{~h}$. The unsupported sample fired at $700{ }^{\circ} \mathrm{C}$ was also ground and used in the XRD tests mentioned above.

The cross-section scanning electron microscopic pictures of these nano-film coated LPFG sensors are shown in Figure 82. Five SCZY-LPFG sensors were tested to be of good quality and sent to MST/Innoventor Inc. for packaging and installation to the gasifier. 


\subsubsection{Determination of $\mathrm{H}_{2}$ sensor operation temperature range}

$\mathrm{H}_{2}$ sensor operation temperature range has been determined based on sensitivity and selectivity for gasification gas monitoring. For both $\mathrm{SrCe}_{0.8} \mathrm{Zr}_{0.1} \mathrm{Y}_{0.1} \mathrm{O}_{3}$ (SCZY) and $\mathrm{SrCe}_{0.95} \mathrm{~Tb}_{0.05} \mathrm{O}_{3-} \quad$ (SCTb) nanofilm-coated LPFG sensors, the ideal temperature range is $500-$ $550^{\circ} \mathrm{C}$ where reasonable sensitivity can be obtained while interference form $\mathrm{H}_{2} \mathrm{~S}, \mathrm{CO}, \mathrm{CO}_{2}, \mathrm{H}_{2} \mathrm{O}$, $\mathrm{CH}_{4}$, and other coexisting gases is negligible.

The gas temperature in the gasifier exiting pipe varies from $\sim 700{ }^{\circ} \mathrm{C}$ at ash remover to $400{ }^{\circ} \mathrm{C}$ at the entrance of gas clean unit. Therefore location of any specific temperature between 400 and $700{ }^{\circ} \mathrm{C}$ can be found in the pipeline for sensor deployment and operation. To identify the best temperature for $\mathrm{H}_{2}$ sensor operation, the SCZY-LPFG and SCTb-LPFG sensor were tested

for $\mathrm{H} 2$ sensing and responses to other gases in a temperature range of 400 to $650{ }^{\circ} \mathrm{C}$. In general, the two types of sensors exhibited similar temperature-dependences of sensitivity and interferences. Figure 83 presents the SCZY-LPFG responses to $\mathrm{H} 2$ at various temperatures as a function of $\mathrm{H}_{2}$ concentration. The sensors have virtually not responses to any gas (including $\mathrm{H}_{2}$ ) at $400^{\circ} \mathrm{C}$ and very small response to $\mathrm{H}_{2}$ at $450^{\circ} \mathrm{C}$. AT $500^{\circ} \mathrm{C}$, the sensors had significant response to $\mathrm{H}_{2}$ with negligible responses to $\mathrm{H}_{2} \mathrm{O}, \mathrm{CO}, \mathrm{CO}_{2}, \mathrm{CH}_{4}$, and $\mathrm{H}_{2} \mathrm{~S}$. The magnitude of $\Delta \lambda_{\mathrm{R}}$ in response to $\mathrm{H}_{2}$ enhanced dramatically as temperature increased to above $550^{\circ} \mathrm{C}$. However, at above $>550^{\circ} \mathrm{C}$, the sensors also start to have large responses to the coexisting gases such as to $\mathrm{H}_{2} \mathrm{O}, \mathrm{CO}, \mathrm{CO}_{2}, \mathrm{CH}_{4}$, and $\mathrm{H}_{2} \mathrm{~S}$. 


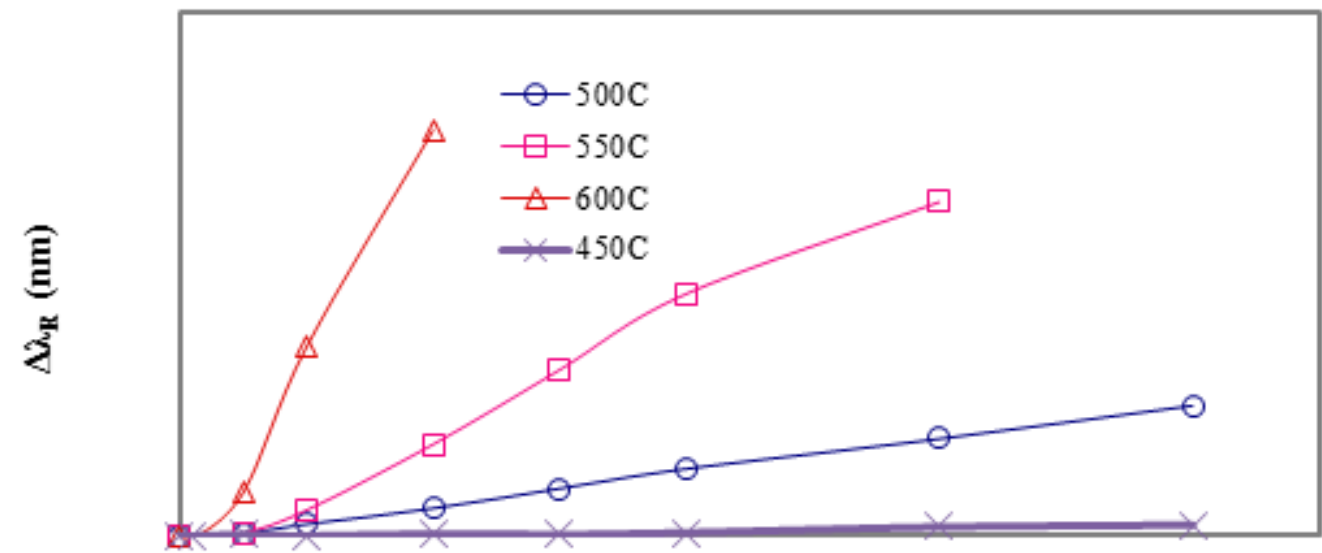

Concentration of $\mathrm{H} 2$ in N2 (\%)

Figure 83 SCZY-LPFG sensor response $\left(\mathrm{Dl}_{\mathrm{R}}\right)$ to bulk $\mathrm{H}_{2}$ in $\mathrm{N}_{2}$ at different temperature (left) and the cross-section SEM image of the sensor tested.

\subsection{High temperature optical sensing with the CDZ-LPFG}

The high temperature optical sensing tests for the CDZ (Cu-doped Zirconia) - LPFG were performed with an apparatus shown in Figure 84, which was the same as that used in our previous work [6]. The CDZ-LPFG segment of the fiber was hosted in a small chamber made of $3 / 8$ " inner diameter stainless steel tube (1/4" in outer diameter), which is placed horizontally in a tubular furnace. The two ends of the stainless tube were connected to the sample gas supply system and ventilation, respectively. The total flow rate of the sample gas was kept at a small value of $10 \mathrm{~cm}^{3} / \mathrm{min}$ at standard temperature and pressure (STP) for maintaining temperature stability and avoiding gas flow caused mechanical disturbance to the suspended fiber sensor. The actual gas temperature in the sensing location was verified by a thermocouple placed inside the tube at the same position of the LPFG segment. The transmission spectrum was obtained by sweeping from 1510 to $1640 \mathrm{~nm}$. The source light was provided by a tunable laser equipped with 
a laser power detector (Agilent 8164A) and a computer data acquisition system. A typical wavelength increment of $0.1 \mathrm{~nm}$ and a dwelling time of $1 \mathrm{~s}$ were used in the spectrum scanning. In the tests of single wavelength response, the transmission laser intensity was read at $10 \mathrm{~Hz}$ frequency.
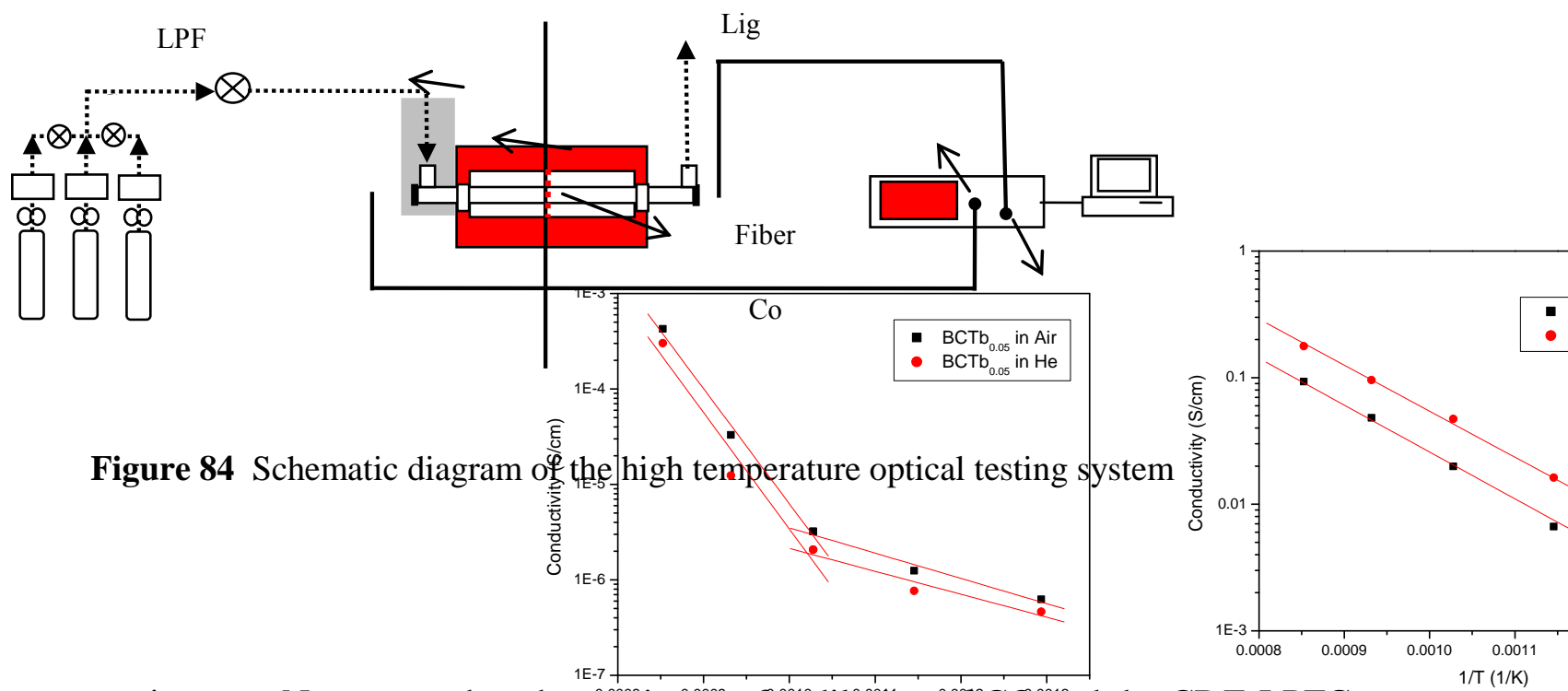

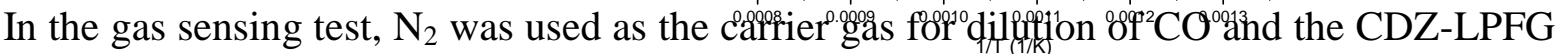

output baseline was measured in pure $\mathrm{N}_{2}$. The baseline $\lambda_{R, N 2}$ is predetermined as a function of temperature represented by the expression of $\left(\lambda_{R, N 2}\right)_{T}=\lambda_{R, N 2}^{0}+\zeta T$ because the $\lambda_{R}$ of LPFGs are known to exhibit excellent linear dependence on temperature. The $\zeta$ value was determined experimentally for each individual CDZ-LPFG as part of the calibration. The typical $\zeta$ values were around $0.11 \mathrm{~nm} /{ }^{\rho} \mathrm{C}$ with small variations among different CDZ-LPFGs. At a specific temperature, the shift of $\lambda_{R}$ in response to switching from $\mathrm{N}_{2}$ to an analyte gas " $i$ " $\left(\Delta \lambda_{R, i}\right)$, is given by:

$$
\Delta \lambda_{R, i}=\left(\lambda_{R, i}-\lambda_{R, N 2}\right)_{T}
$$


where $\lambda_{R, i}$ is the resonant wavelength in gas $i$ at temperature $T$. The response speed of the CDZLPFG in gas sensing was studied by measuring the temporal evolution of the transmission light intensity at a fixed wavelength. The response time is represented by the time required for stabilizing the output light intensity at the fixed wavelength. For each temperature, the wavelength corresponding to $\sim 60 \%$ of the peak height is specifically chosen as the fixed wavelength for operation so that high sensitivity can be obtained for detecting variation of transmission light intensity.

\subsubsection{CDZ film and properties}

The high temperature optical sensing tests for the CDZ-LPFG were performed. The CDZLPFG segment of the fiber was hosted in a small chamber made of $3 / 16^{\prime \prime}$ inner diameter (1/4" outer diameter) stainless steel tube, which was placed horizontally in a tubular furnace. The two ends of the stainless tube were connected to the sample gas supply system and ventilation, respectively. The total flow rate of the sample gas was kept at a small value of $10 \mathrm{~cm}^{3} / \mathrm{min}$ at standard temperature and pressure (STP) for maintaining temperature stability and avoiding gas flow caused mechanical disturbance to the suspended fiber sensor. The actual gas temperature in the sensing location was verified by a thermocouple placed inside the tube at the same position of the LPFG segment. The sensor spectrum was acquired using a tunable laser (Agilent 81640A) and an optical power meter (Agilent 81633A). A host computer controlled the tunable laser sweeping from 1510 to $1640 \mathrm{~nm}$ and the power meter at each wavelength to reconstruct the transmission spectrum recorded the power. A typical wavelength increment of $0.1 \mathrm{~nm}$ and a dwelling time of $1 \mathrm{~s}$ were used in the spectrum scanning. In the tests of single wavelength response, the transmission laser intensity was read at $10 \mathrm{~Hz}$ frequency. 
The polymeric precursor was examined by Attenuated Total Reflectance (ATR) FTIR in a wavenumber range from 400 to $4000 \mathrm{~cm}^{-1}$. Figure 85 presents the FTIR spectra of the metal ion-containing polyethylene glycol (Metal-PEG) in comparison with those of the ethylene glycol (EG) monomer and metal-free polyethylene glycol (PEG). For the $\mathrm{Zr}^{4+}$ and $\mathrm{Cu}^{2+}$ incorporated PEG, distinct absorptions at $650 \mathrm{~cm}^{-1}, 912 \mathrm{~cm}^{-1}, 1160 \mathrm{~cm}^{-1}$, and $1750 \mathrm{~cm}^{-1}$ are found in MetalPEG but not existent in the EG monomer and metal-free PEG. The peaks at $1160 \mathrm{~cm}^{-1}$ and 1750 $\mathrm{cm}^{-1}$ are assigned to the $\mathrm{C}=\mathrm{O}$ double bond which would not be stable in the pure $\mathrm{PEG}$ without the associating oxygen with cations. The noticeable absorptions at $650 \mathrm{~cm}^{-1}, 912 \mathrm{~cm}^{-1}$ indicate the weakly bonding between the metal ions and oxygen. The FTIR results confirm the chelated metal ion in the metal-PEG precursor that is consistent with the literature report [14].

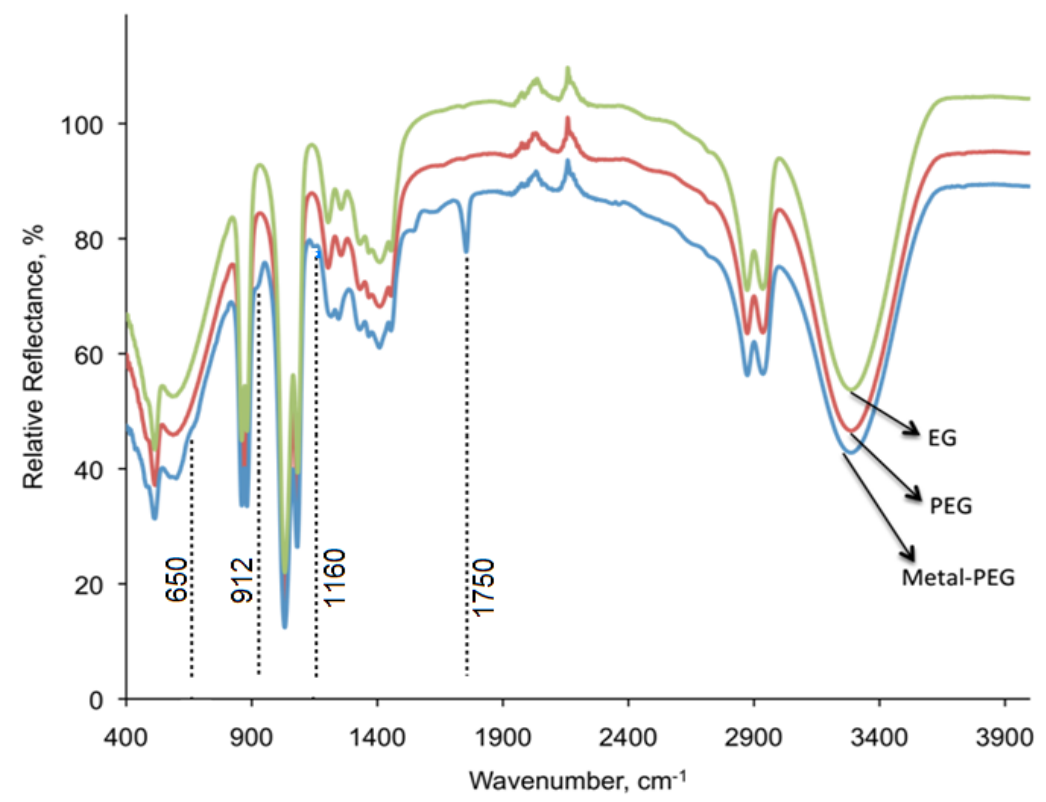

Figure 85 FTIR spectra of ethyl glycol (EG), poly ethyl glycol (PEG) metal-chaleted PEG (MetalPEG). 
Figure 86 (a) shows the SEM images of the cured polymeric precursor film on the optical fiber obtained by brush coating. The precursor film had a uniform thickness of $\sim 500 \mathrm{~nm}$ which resulted in a metal oxide film thickness of $50-60 \mathrm{~nm}$ after firing. Figure 86 (b) and Figure 86 (c) show the SEM images of the cross-section and surface of the CDZ-LPFG obtained by four cycles of coating and firing followed by a final step of annealing at $750^{\circ} \mathrm{C}$ for $30 \mathrm{~min}$. The fourtime coated CDZ film on the fiber had a thickness of $220-250 \mathrm{~nm}$. TEM examinations of the CDZ particles prepared by identical thermal treatments revealed that the CDZ grain size was 20 - $25 \mathrm{~nm}$. The CDZ grain size remained virtually unchanged after $96 \mathrm{~h}$ of further annealing at $550^{\circ} \mathrm{C}$ as shown by the insert in Figure 86 (c). The thermal stability of the CDZ-LPFG at $550^{\circ} \mathrm{C}$ was improved dramatically by the thermal treatment at $750^{\circ} \mathrm{C}$. Without this final thermal treatment, $\lambda_{\mathrm{R}}$ of the CDZ-LPFG drifted $0.4 \mathrm{~nm}$ towards shorter wavelength at $550^{\circ} \mathrm{C}$ in just two hours. In contrast, the CDZ-LPFG received 30 -min annealing at $750^{\circ} \mathrm{C}$ had no measurable drift at $550^{\circ} \mathrm{C}$ in a two-hour period of test. The $\lambda_{R}$ of the final CDZ-LPFG shifts with temperature at a rate of $\zeta=0.114 \mathrm{~nm} /{ }^{\circ} \mathrm{C}$ within a temperature range of $350-600{ }^{\circ} \mathrm{C}$.

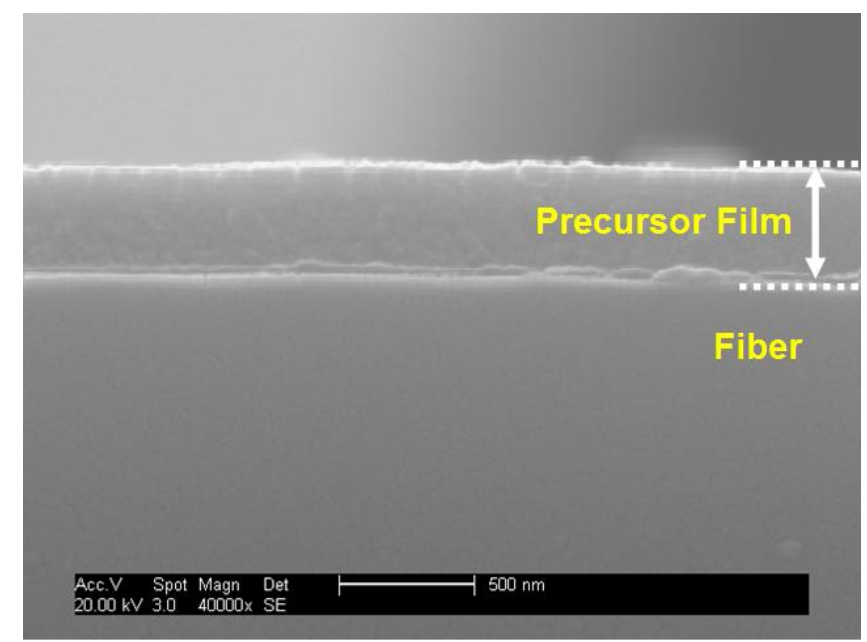

(a) 


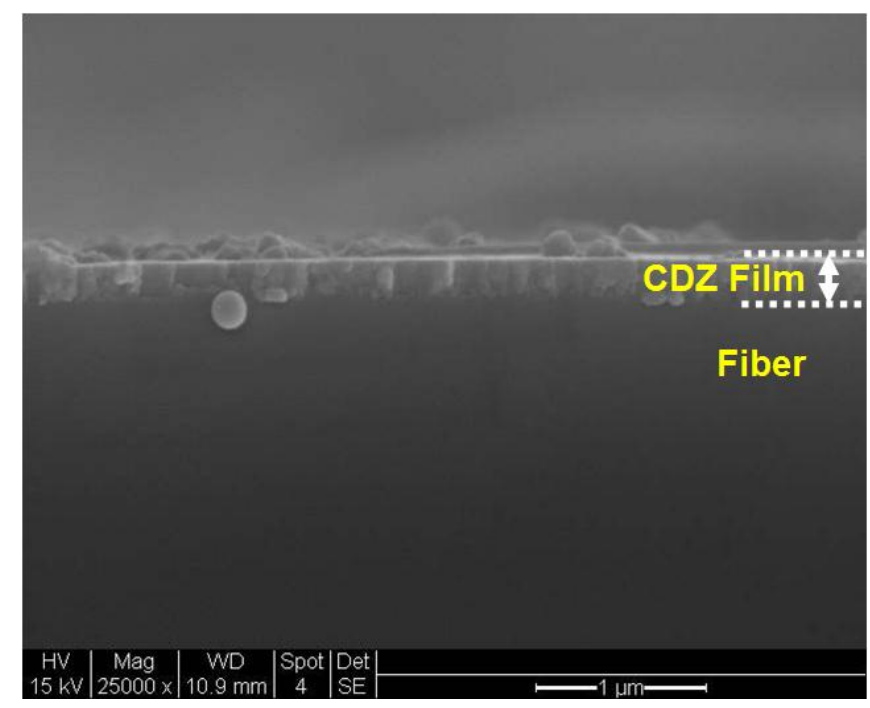

(b)

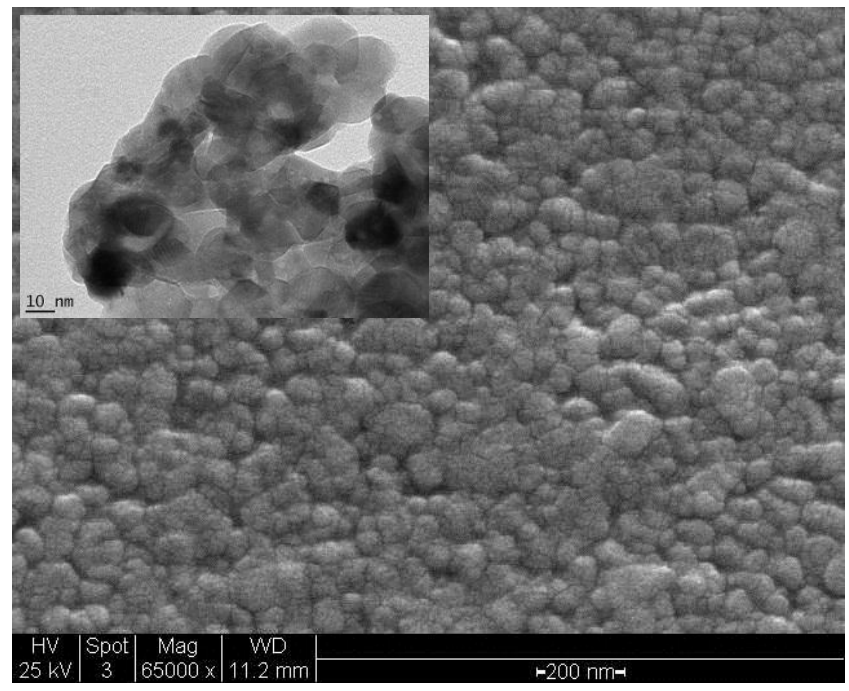

(c)

Figure 86 SEM pictures of (a) cross-section of a single time coated precursor film after drying, (b) cross-section of the four-time coated CDZ film on fiber, and (c) surface of the CDZ film on fiber with insert showing TEM picture of CDZ particles after $96 \mathrm{~h}$ of annealing at $550^{\circ} \mathrm{C}$. 
Energy dispersive X-ray spectroscopic (EDS) test confirmed that the $\mathrm{Zr}$ :Cu ratio in the synthesized oxides was $\sim 16: 84$, same as that in the starting precursor solution. The XRD pattern of the CDZ nanoparticles clearly shows a two-phase composition of tetragonal zirconia $\left(\mathrm{T}-\mathrm{ZrO}_{2}\right)$ and monoclinic zirconia $\left(\mathrm{M}-\mathrm{ZrO}_{2}\right)$. The observed two phase composition is in agreement with the literature findings on phase compositions of $\mathrm{Cu}$-doped zirconia. The formation of monoclinic phase occurs when $\mathrm{CuO}$ incorporates into pure tetragonal zirconia or Y-doped zirconia [15]. Figure 87 presents the XRD spectrum of the $16 \% \mathrm{Cu}$-doped $\mathrm{ZrO}_{2}$ where only strong peaks of T$\mathrm{ZrO}_{2}$ and $\mathrm{M}-\mathrm{ZrO}_{2}$ peaks are indentified without peaks of copper oxides. The formation of the CDZ solid solution involves $\mathrm{CuO}$ incorporation into the $\mathrm{ZrO}_{2}$ lattice that can be described by the Kroger-Vink notations as:

$\mathrm{CuO} \underset{\mathrm{ZrO}_{2}}{\circledR} \mathrm{Cu}_{\mathrm{Zr}}^{\prime \prime}+\mathrm{V}_{O}^{\bullet \bullet}+\mathrm{O}_{O}^{X}$

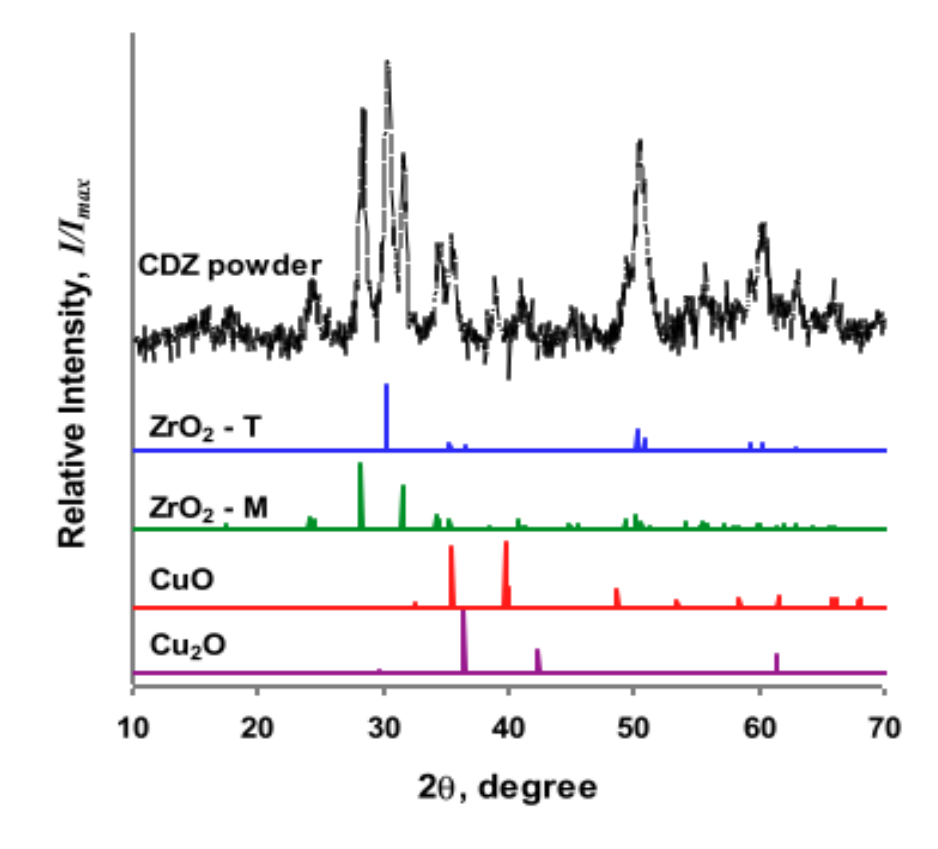

Figure $87 \mathrm{XRD}$ pattern of the $\mathrm{CDZ}$ particles together with standard patterns of $\mathrm{T}-\mathrm{ZrO} 2, \mathrm{M}-\mathrm{ZrO} 2$, $\mathrm{CuO}$ and $\mathrm{Cu}_{2} \mathrm{O}$. 
The refractive index was obtained by ellipsometry test for the CDZ nanocrystalline thin film supported on silicon wafer. The refractive index of the CDZ film $\left({ }^{n_{C D Z}}\right)$ decreases slightly and linearly from 1.53 to 1.51 and the extinction coefficient decreases from 0.27 to 0.22 as wavelength increases from 1510 to $1640 \mathrm{~nm}$.

\subsubsection{CDZ-LPFG response to CO}

Figure 88 shows the transmission spectra of the LPFG before and after CDZ film coating at room temperature and the spectrum of the CDZ-LPFG at $550^{\circ} \mathrm{C}$.

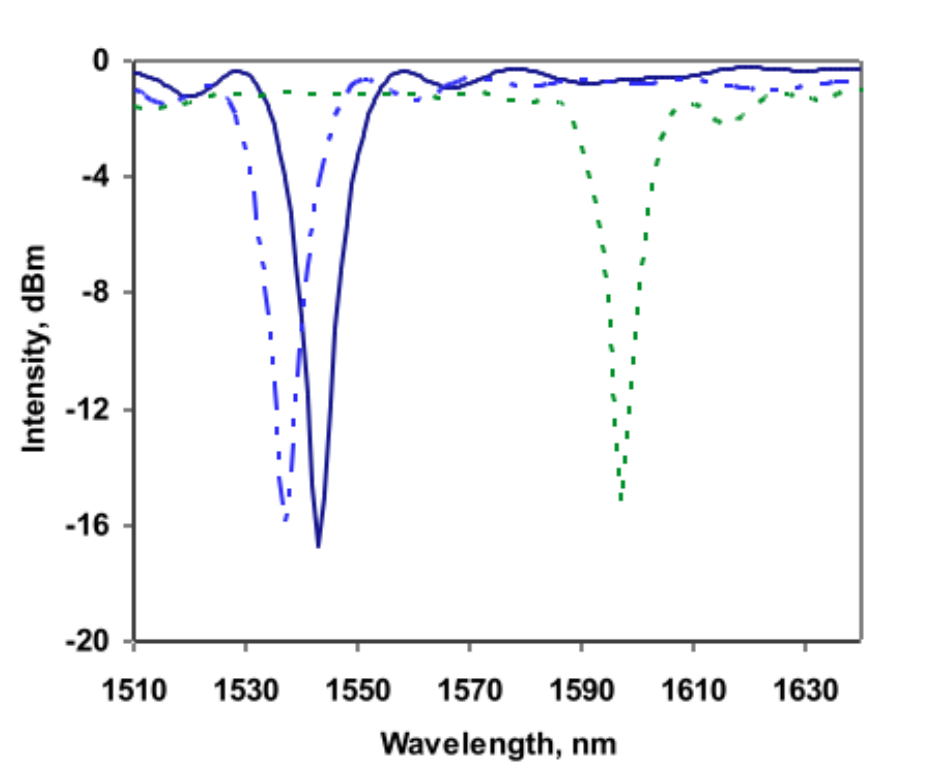

Figure 88 The transmission spectra of the LPFG before and after coating CDZ. 
The Cu-doped CDZ-LPFG was first examined for its optical response to switching between air and a gas mixture of 1,000 ppmv $\mathrm{CO}$ in $\mathrm{N}_{2}$. Before recording the response, the CDZLPFG was stabilized at $550^{\circ} \mathrm{C}$ for $72 \mathrm{~h}$, including $48 \mathrm{~h}$ in air and then $24 \mathrm{~h}$ in the $1,000 \mathrm{ppm} \mathrm{CO}$. Figure 89 (a) presents the transmission spectra in air and 1,000 ppmv $\mathrm{CO}$ in a wavelength range around the resonant peaks. The spectrum responded to the $\mathrm{CO}$ gas mixture with a red shift $\left(\Delta \lambda_{R}\right.$ $>0$ ). According to the pseudo two-layer model for the case of $n_{o v}>n_{\text {clad, }}{ }^{\text {[Hou et al., 2001] }}$ the red shift of $\lambda_{R}$ suggests that a decrease of $n_{C D Z}$ occurred in the CO-containing gas at high temperature. Figure 89 (b) shows the temporal evolution of the transmission light intensity at a fixed wavelength of $1610 \mathrm{~nm}$ during switching between the two gas flows at $550^{\circ} \mathrm{C}$. The excellent reversibility of optical responses to the two gases indicates that the CDZ-LPFG is capable of sensing CO gas. The response speed was faster for the oxidizing process $(<1 \mathrm{~min})$ than for the reverse reducing process $(\sim 8 \mathrm{~min})$.

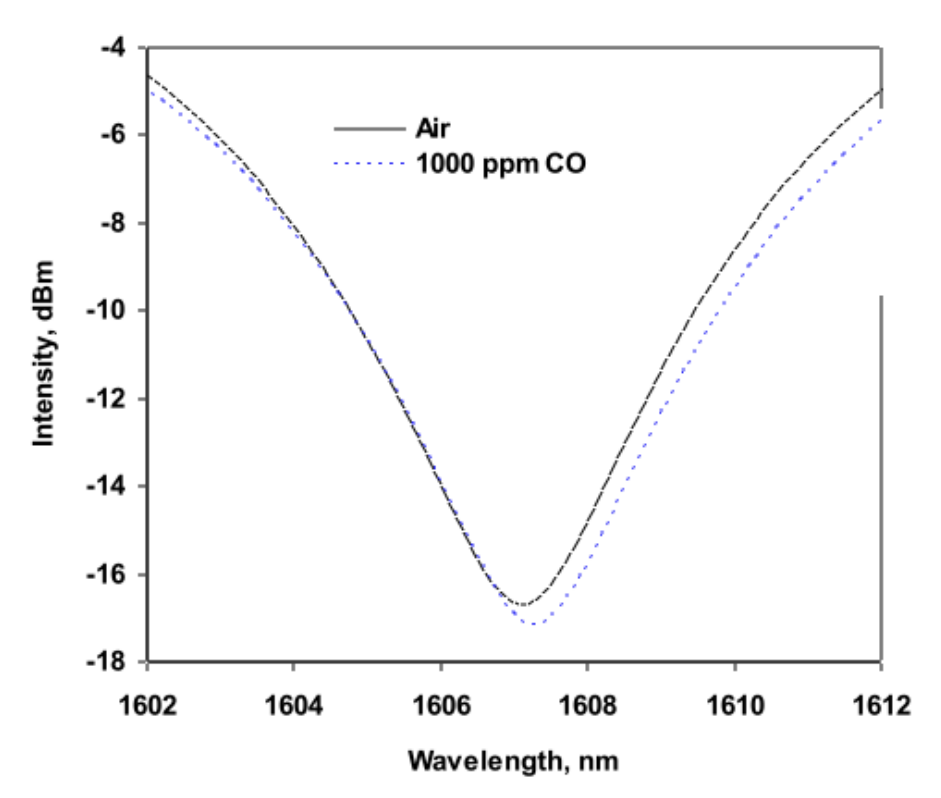

(a) 


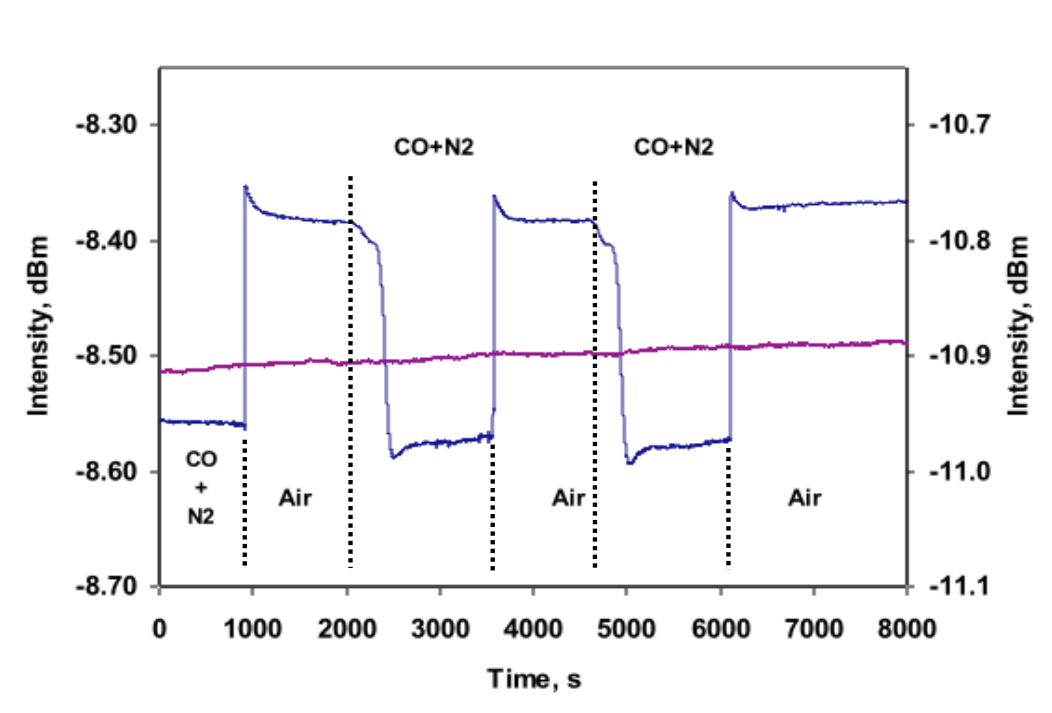

(b)

Figure 89 The CDZ-LPFG response to switching between air and 1,000 ppmv CO in N2 at 550oC: (a) transmission spectra in the two gases and (b) the single wavelength $(\lambda=1610 \mathrm{~nm})$ temporal responses to gas switching (arrows indicating gas switching points).

For comparison, an yttrium-stabilized-zirconia (YSZ) thin film coated LPFG (YSZ-LPFG) was also fabricated under the same conditions as described above. The temporal response of the YSZ-LPFG to the 1,000 ppmv CO and air was measured and the results are presented in Figure 6 (b) on the same scale of intensity. The YSZ-LPFG was virtually irresponsive to the switching between air and 1,000 ppmv $\mathrm{CO}$ containing $\mathrm{N}_{2}$ because both $\mathrm{Y}^{3+}$ and $\mathrm{Zr}^{4+}$ are not readily reduced in the low concentration $\mathrm{CO}$ at the temperature of $550^{\circ} \mathrm{C}$. This suggests that the $\mathrm{Cu}$ dopant enhances the red-ox activities for the CDZ material that is crucial to obtaining high sensitivity for low concentration $\mathrm{CO}$ detections.

The optical responses of the CDZ-LPFG to $\mathrm{CO}$ and air are results of the varying refractive index of the CDZ coating $\left(n_{C D Z}\right)$ in different atmospheres. The causes of the $n_{C D Z}$ changes are 
due primarily to the following gas-solid reactions. At high temperature, reduction of $\mathrm{Cu}^{2+}$ to $\mathrm{Cu}^{+}$ occurs in the solid phase when $\mathrm{CDZ}$ contacts $\mathrm{CO}$,

$$
\mathrm{CO}+2 \mathrm{Cu}_{\mathrm{Zr}}^{\prime \prime}\left(\mathrm{Cu}^{2+}\right)+\mathrm{O}_{O}^{X}{ }^{\circledR} 2 \mathrm{Cu}_{\mathrm{Zr}}^{\prime \prime \prime}\left(\mathrm{Cu}^{+}\right)+\mathrm{V}_{\mathrm{O}}^{\bullet \bullet}+\mathrm{CO}_{2}
$$

When the reduced $\mathrm{CDZ}$ is re-exposed to air, $\mathrm{Cu}^{+}$is oxidized back to $\mathrm{Cu}^{2+}$ as the reverse of reaction (2) happens in the solid phase,

$$
\frac{1}{2} \mathrm{O}_{2}+2 C u_{Z r}^{\prime \prime \prime}\left(C u^{+}\right)+V_{O}^{\bullet \bullet} 2 C u_{Z r}^{\prime \prime}\left(C u^{2+}\right)+O_{O}^{X}
$$

When the oxygen partial pressure is high, further oxidation may occur at the oxygen vacancies created by $\mathrm{Cu}$-doping in reaction (1), which provides the well-known oxygen ion conductivity in doped-zirconia materials,

$$
\frac{1}{2} O_{2}+V_{O}^{\bullet \bullet} O_{O}^{X}+2 h^{\bullet}
$$

The repeating pattern of the CDZ-LPFG optical output in Figure 89 indicates that reactions (2), (3) and (4) occur reversibly when switching between the 1,000 ppmv CO and air.

Experiment was also performed to examine the optical response of the CDZ-LPFG to a much higher CO concentration of 1vol\%. The optical output of the CDZ-LPFG exhibited an anomalous but still repeating pattern in response to switching between the $1 \mathrm{vol} \% \mathrm{CO}$ and air after the first cycle. As shown in Figure 90(a), in the initial contact with 1vol\% CO, the temporal response of transmission light intensity showed a trend similar to that observed in the 1,000 ppmv CO but with much slower stabilizing process. However, the single wavelength response exhibited a different repeating pattern afterwards when switching between the 1 vol\% $\mathrm{CO}$ and air. In the 1vol\% CO atmosphere, the transmission light intensity first decreased to a minimum and then increased dramatically before stabilizing. Furthermore, the decrease of transmission intensity over time was far more severe than the normal thermal drifting, suggesting that factors other than 
the normal thermal instability have caused faster drift of CDZ-LPFG spectrum in the presence of 1vol\% CO.

To investigate causes of this anomalous behavior in high concentration $\mathrm{CO}$, a fresh $\mathrm{CDZ}$ LPFG was used to record the transmission spectra at different times qualitatively corresponding to points A, B, and C in Figure 90 (a). The resultant spectra are shown in Figure 90 (b). It was found that, in the $1 \mathrm{vol} \% \mathrm{CO}$ environment, the resonant peak first shifted to longer wavelength and then swung back to shorter wavelength. This spectrum swing causes the single wavelength light transmission intensity to first decrease, then increase with time as observed in Figure 90(a).

The implication of such a two-stage shift of $\lambda_{\mathrm{R}}$ in opposite directions is that the ${ }^{n_{C D Z}}$ first decreased and then increased ${ }^{\text {[Zhu et al. 2001] }}$ with contacting time in high concentration CO gas. A silicon wafer-supported $\mathrm{CDZ}$ film was treated at $550^{\circ} \mathrm{C}$ for three hours in the high concentration $\mathrm{CO}$ and then cooled down in the same gas flow. The ${ }^{n_{C D Z}}$ of this CO-treated CDZ film was around 1.92 at room temperature in the wavelength range of $1510-1640 \mathrm{~nm}$ which was indeed much greater than that of the untreated film (1.53 1.51), though the $n_{C D Z}$ value at high temperature could be quantitatively different. 


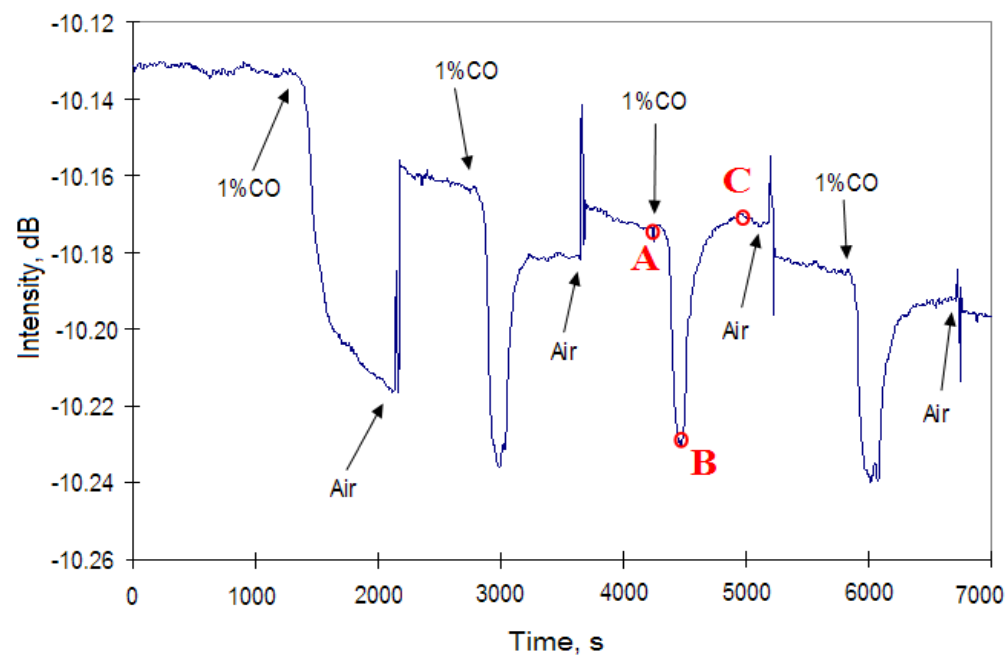

(a)

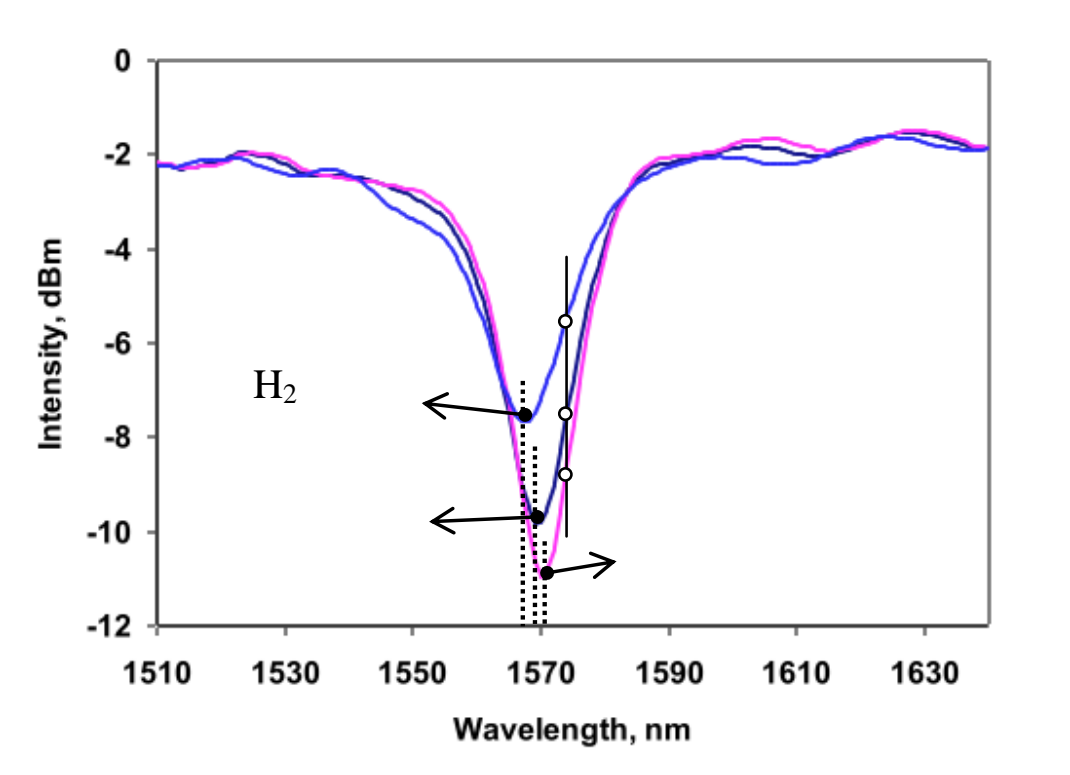

(b)

Figure 90 (a) The CDZ-LPFG single wavelength $(\lambda=1576 \mathrm{~nm})$ response to switch between air and $1 \mathrm{vol} \% \mathrm{CO}$ in $\mathrm{N} 2$ at $550 \mathrm{oC}$ (arrows indicating points of gas switch) and (b) the transmission spectra at different times in contact with 1 vol\% CO. 


\subsubsection{CDZ stability in CO}

The very different optical responses of the CDZ-LPFG to $1,000 \mathrm{ppmv}$ and $1 \mathrm{vol} \% \mathrm{CO}$ indicate that solid phase changes other than reaction (2) are involved under high concentration of CO. XRD was performed to examine the $16 \% \mathrm{Cu}$-doped $\mathrm{CDZ}$ nano crystalline particles after exposing to different concentrations of $\mathrm{CO}$ at $550^{\circ} \mathrm{C}$. The particle sample after being treated at $550^{\circ} \mathrm{C}$ in a specific gas stream was cooled to room temperature with the testing gas flowing continuously. Figure 91 shows the XRD patterns of the CDZ sample after treatments in 400 ppmv CO and air. The CDZ crystal phase composition was found to be essentially unchanged after multiple cycles of alternating treatments in 400 ppmv $\mathrm{CO}$ and air. This demonstrates that the crystalline phase of the CDZ film is quite stable in low concentration $\mathrm{CO}(<1,000 \mathrm{ppmv})$ at $550^{\circ} \mathrm{C}$

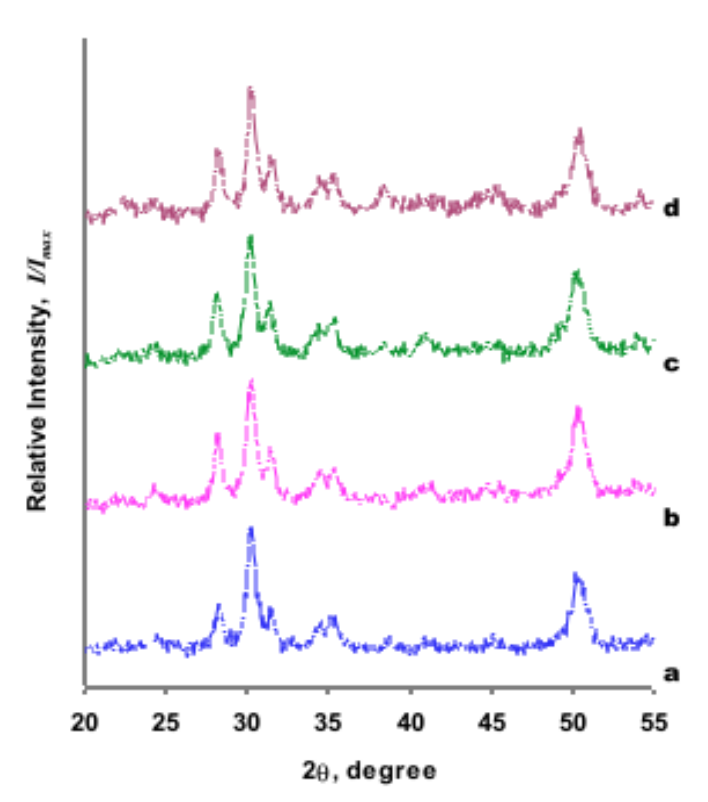

Figure $91 \mathrm{XRD}$ spectra of the $\mathrm{CDZ}$ particles after being treated at $550 \mathrm{oC}$ in: $\mathrm{a}-$ fresh sample; $\mathrm{b}-2 \mathrm{~h}$ in 400 ppmv CO; c - $2 \mathrm{~h}$ in air; $\mathrm{d}$ - after four cycles of switching between air and $400 \mathrm{ppmv} \mathrm{CO}$ with a final step of $10 \mathrm{~h}$ in $400 \mathrm{ppmv} \mathrm{CO}$. 
However, after $2 \mathrm{~h}$ of treatment in the $1 \mathrm{vol} \% \mathrm{CO}$, the characteristic peak of copper metal $\left(\mathrm{Cu}^{0}\right)$ appeared in the XRD spectrum as shown in Figure 92. This indicates that further reduction of $\mathrm{Cu}^{+}$to $\mathrm{Cu}^{0}$ occurs at $550^{\circ} \mathrm{C}$ in high concentration $\mathrm{CO}$ (e.g. 1 vol\%) and the metallic $\mathrm{Cu}$ species was segregated from the solid oxide phase to grain boundaries.

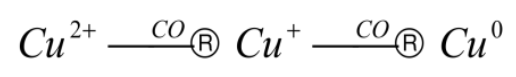

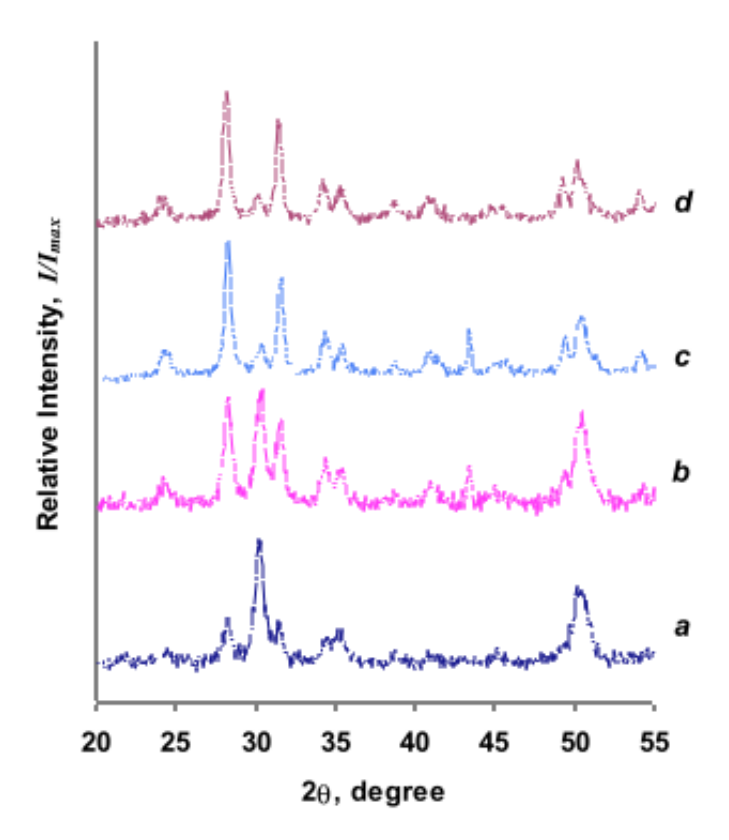

Figure $92 \mathrm{XRD}$ spectra of the CDZ particles after being treated at $550 \mathrm{oC}$ : a - fresh sample, $\mathrm{b}-$ in $1 \mathrm{vol} \% \mathrm{CO}$ for $2 \mathrm{~h}, \mathrm{c}-$ after three cycles of switching between air and $1 \mathrm{vol} \% \mathrm{CO}$ ending with $2 \mathrm{~h}$ in 1 vol\% $\mathrm{CO}, \mathrm{d}-$ after $2 \mathrm{~h}$ in air.

The $\mathrm{Cu}$ peaks disappeared after subsequent treatment in air for $2 \mathrm{~h}$ due to oxidation but the characteristic XRD peaks of copper oxides were not found, likely because of $\mathrm{CuO}$ dissolution back to the zirconia phase. The readiness of $\mathrm{CuO}$ dissolution into doped zirconia materials has been well documented in the literature. The $\mathrm{Cu}$ incorporation into the tetragonal yttrium 
stabilized $\mathrm{ZrO}_{2}$ phase has been reported to happen at $>850^{\circ} \mathrm{C}$ which causes transition to $\mathrm{M}-\mathrm{ZrO}_{2}$ phase. Our previous research also revealed that, for nanoparticles of zirconia and metal oxides, formation of solid solution can occur at temperatures lower that for coarsen particle systems . The reincorporation of $\mathrm{CuO}$ nano-clusters from the grain boundaries into the zirconia structure is possible to take place at $550^{\circ} \mathrm{C}$. The $\mathrm{Cu}$ metal reappeared after the sample was treated in the $1 \mathrm{vol} \% \mathrm{CO}$ gas again. It was also observed that the relative intensities of the $\mathrm{M}-\mathrm{ZrO}{ }_{2}$ continued to grow while those of the $\mathrm{T}-\mathrm{ZrO}_{2}$ decreased during the process of switching gas flows. This indicates that a tetragonal-to-monoclinic phase transformation was facilitated by the reduction of copper ions and segregation of copper from the crystal structure in the $1 \mathrm{vol} \% \mathrm{CO}$.

HRTEM was employed to examine the microstructure of the CDZ nanoparticles before and after the treatment in $1 \mathrm{vol} \% \mathrm{CO}$ at $550{ }^{\circ} \mathrm{C}$. The fresh $\mathrm{CDZ}$ nanoparticles were of high crystallinity and had an average grain size of $25-30 \mathrm{~nm}$ as shown by the TEM picture in Figure 93 (a). The electron diffraction pattern of the fresh CDZ in Figure 93 (b) represents a two-phase nanocrystalline mixture as expected. A single crystalline pattern for $\mathrm{M}-\mathrm{ZrO}_{2}$ is distinguishable in Figure 93 (b) because of the very small sample size. After high temperature treatment in the $1 \mathrm{vol} \% \mathrm{CO}$, areas of distorted lattice and numerous crystalline domains with size around 5 10 are seen in the CDZ nanoparticles (Figure 93 (c)). It is obvious that the large number of cation vacancies resulted from $\mathrm{Cu}^{2+}$ reduction to $\mathrm{Cu}^{0}$ has caused collapse of the crystal structures. The electron diffraction pattern of the reduced sample in Figure 93 (d) is characteristic of a polycrystalline structure because of the randomly oriented small crystalline islands. 


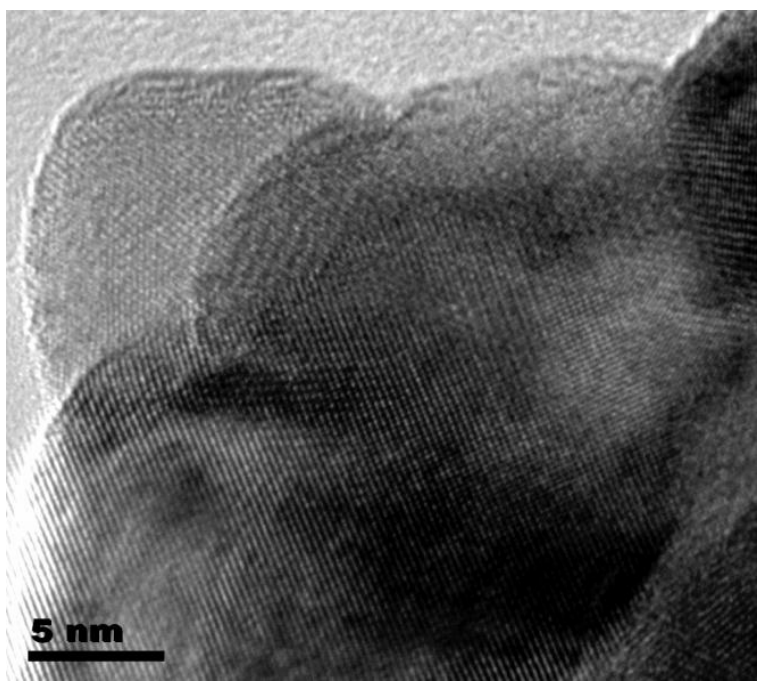

(a)

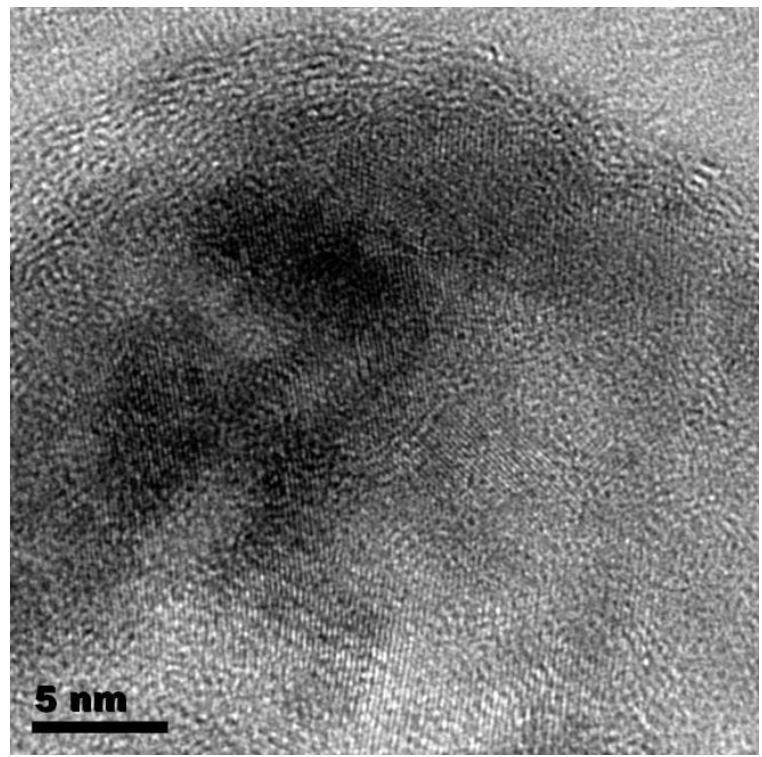

(c)

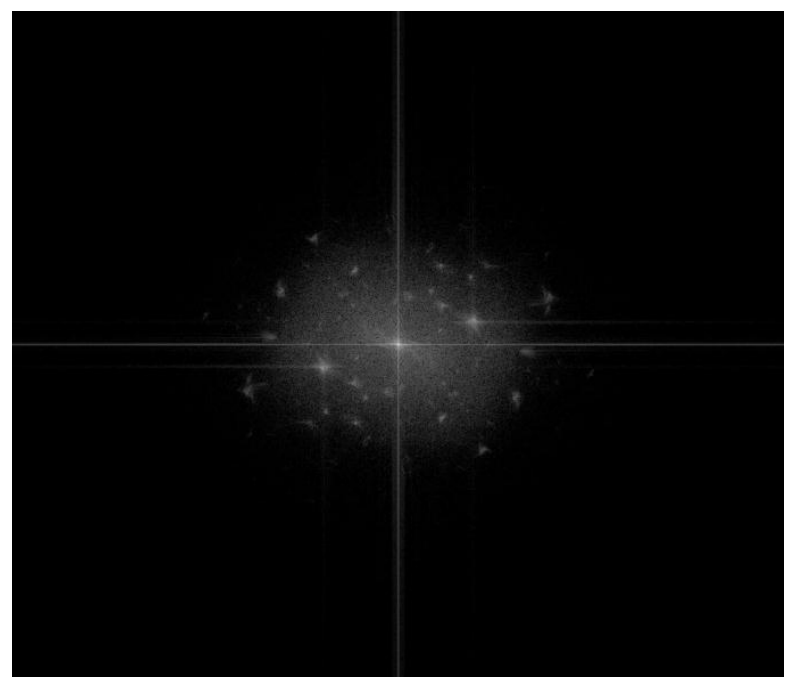

(b)

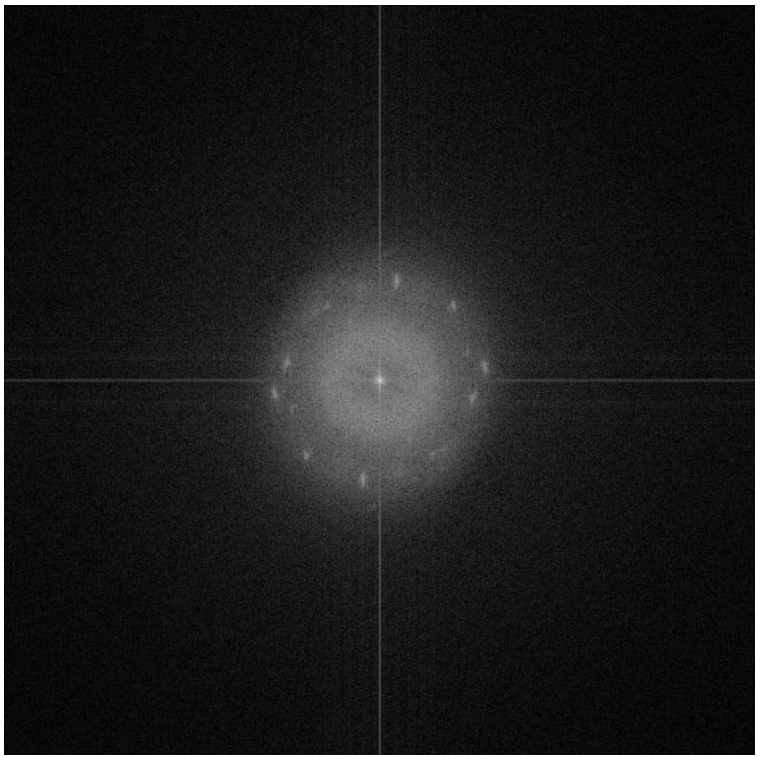

(d)

Figure 93 TEM images and electron diffraction patterns of the Cu-doped CDZ nanoparticles: (a) and (b) - fresh CDZ particle; (c) and (d) after reduction in 1vol\% CO for $10 \mathrm{~h}$. 
Although $\mathrm{Cu}$ metal nano particles were not found by TEM examination, the $\mathrm{Cu}$ migration from the center of $\mathrm{CDZ}$ nano particle to the surface in high concentration $\mathrm{CO}$ environment is evidenced by the EDX line scanning results. Figure 94 presents the distributions of $\mathrm{Zr}$ and $\mathrm{Cu}$ contents given by atomic percentages along the line through the crystallites before and after treatment in the $1 \mathrm{vol} \% \mathrm{CO}$ gas flow at $550^{\circ} \mathrm{C}$. The fresh $\mathrm{CDZ}$ particle had a uniform $\mathrm{Cu}$ content throughout the particle while the $\mathrm{Cu}$ content was much high at the edge than in the center after being exposed to 1 vol\% CO. Thus, the $16 \% \mathrm{Cu}$-doped zirconia is chemically unstable in high temperature and high concentration $\mathrm{CO}$ environments. The $\mathrm{Cu}$ migration together with the dramatic solid structure evolution is likely responsible for the overall fast drifting of the CDZLPFG transmission light intensity in 1vol\% CO. 


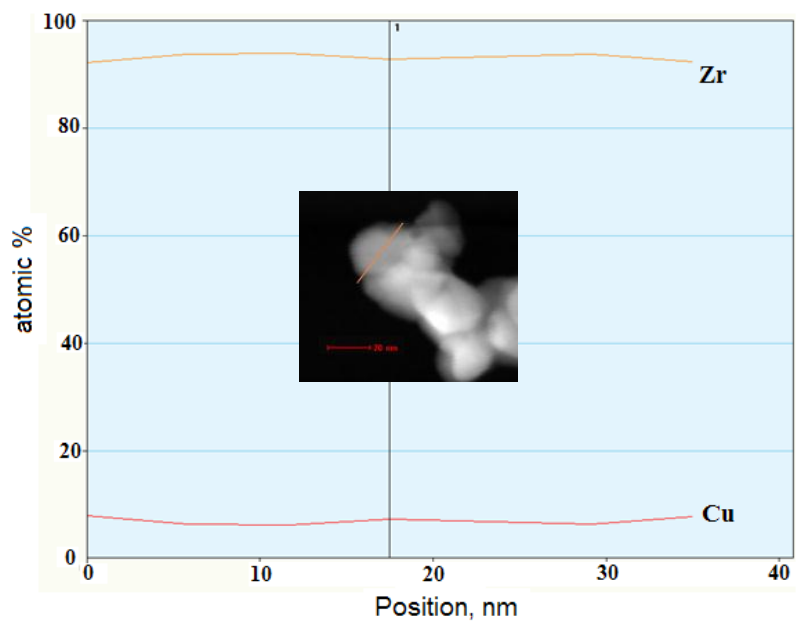

(a)

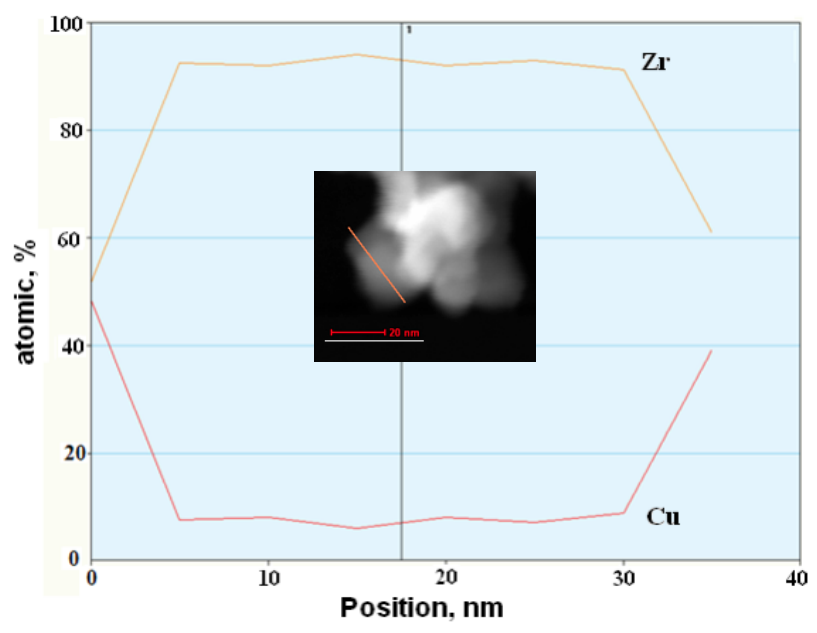

(b)

Figure $94 \mathrm{Zr}$ and $\mathrm{Cu}$ content distributions measured by EDX line scanning for (a) fresh CDZ nanoparticle and (b) CDZ nanoparticles after $3 \mathrm{~h}$ of treatment in 1vol\% CO at 550oC (inserts are TEM images showing the scanning lines position).

\subsubsection{Low concentration CO measurement}

The results of the above studies on the stability of CDZ nano particles suggest that the $16 \% \mathrm{Cu}$-doped CDZ-LPFG is potentially useful for high temperature detection of low concentration $\mathrm{CO}$ but inefficient for measurement of high concentration CO. The CDZ-LPFG 
was thus tested for its response to $250 \mathrm{ppmv} \mathrm{CO}$ in $\mathrm{N}_{2}$ in a temperature range of $350-600^{\circ} \mathrm{C}$. The CDZ-LPFG did not show appreciable response at up to $400^{\circ} \mathrm{C}$. It started to show a measurable $\Delta \lambda_{R}$ of $0.05 \mathrm{~nm}$ at $450^{\circ} \mathrm{C}$. The $\Delta \lambda_{R}$ increased drastically from $0.05 \mathrm{~nm}$ to 0.33 when temperature rose from $450^{\circ} \mathrm{C}$ to $500^{\circ} \mathrm{C}$ and then changed only slightly when temperature further rose to $550^{\circ} \mathrm{C}\left(\Delta \lambda_{R}=0.36 \mathrm{~nm}\right)$ and $600^{\circ} \mathrm{C}\left(\Delta \lambda_{R}=0.35 \mathrm{~nm}\right)$. At $650^{\circ} \mathrm{C}$, the resonant wavelength $\left(\lambda_{R}\right)$ shifted outside the instrument's operating limit of $1640 \mathrm{~nm}$. This temperature-dependence of the CDZ-LPFG response to $\mathrm{CO}$ reveals that the red-ox reactions of the doped-copper is sufficiently activated only at $>450^{\circ} \mathrm{C}$, which is much higher than the temperature required for the stepwise reduction in equation (5) to happen in pure copper oxide.

Optical sensing test was therefore performed in a $\mathrm{CO}$ concentration range from 0 to 1,000 ppmv at $550^{\circ} \mathrm{C}$ where the CDZ-LPFG has high sensitivity to $\mathrm{CO}$ but exhibits only very minor thermal drift. Figure 95 (a) presents the spectra in $\mathrm{N}_{2}$ containing $\mathrm{CO}$ of 0 ppmv, 249 ppmv, and 1036 ppmv, respectively. The transmission spectrum and thus the $\lambda_{\mathrm{R}}$ continuously shifted toward longer wavelength with increasing the $\mathrm{CO}$ concentration. The sensing results are presented in Figure 95 (b) by plotting the $\Delta \lambda_{R}$ versus CO concentration. The CDZ-LPFG reposes exhibited a strong correlation between the $\Delta \lambda_{\mathrm{R}}$ and CO-concentration that is promising for quantification of ppm-level CO concentration at high temperatures. 


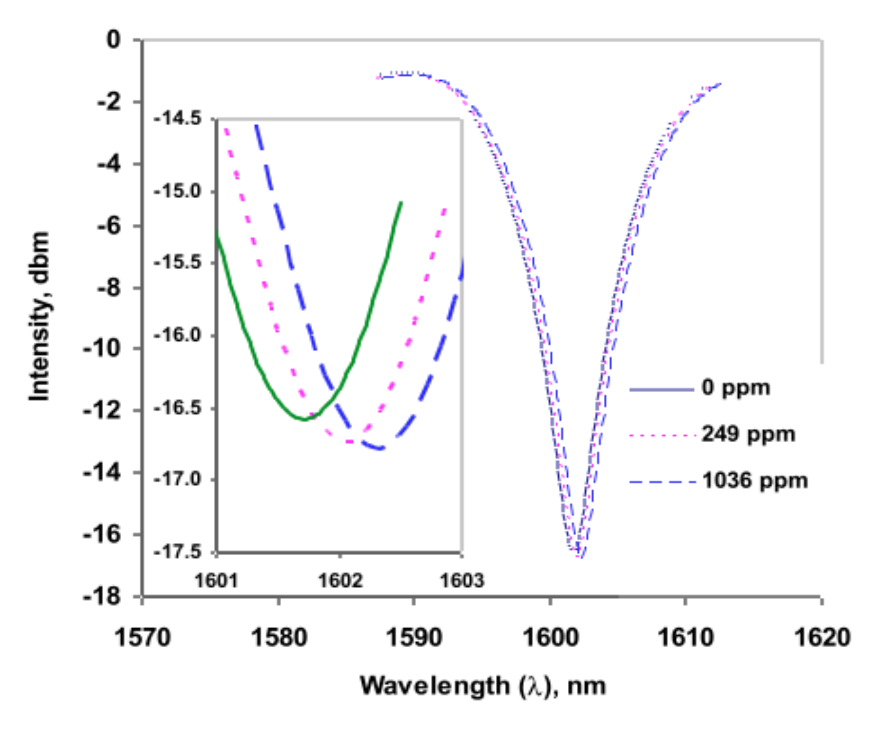

(a)

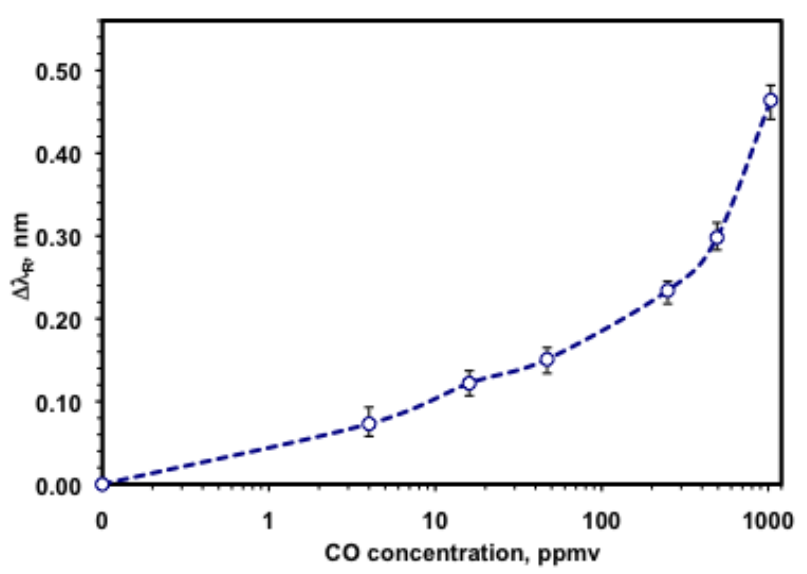

(b)

Figure 95 CDZ-LPFG transmission spectra gases of different $\mathrm{CO}$ concentrations (a) and sensor output $\Delta \lambda \mathrm{R}$ as a function of $\mathrm{CO}$ concentration at $550^{\circ} \mathrm{C}(\mathrm{b})$.

Because the crystal phase and grain size of the CDZ film are essentially unchanged in the testing condition of low CO concentrations, the $n_{C D Z}$ variation is due primarily to the population changes of the lattice elements involved in reaction (2). The changes in $\mathrm{Cu}$ dopant states 
$\left(\mathrm{Cu}^{2+} \mathrm{Cu}^{+}\right)$and associated variation in ${ }^{\left[V_{O}^{\bullet \bullet}\right]}$ affect the electrical properties [Ruiz-Morales et al., 2008] and the density of the CDZ material that consequentially varies the $n_{C D Z}$ value. Based on the reaction thermodynamics, $\left[\mathrm{Cu}^{+}\right]$and $\left[V_{O}^{\bullet \bullet}\right]$ increase with the concentration or partial pressure of the reactant $\mathrm{CO}$, the trend of growing $\lambda_{R}$ red shift with $\mathrm{CO}$ concentration implies that $n_{C D Z}$ decreases with increasing $\left[\mathrm{Cu}^{+}\right]$and $\left[V_{O}^{\bullet \bullet}\right]$.

The polymeric precursor route was employed to synthesize nano crystalline $\mathrm{Cu}$-doped zirconia (CDZ) thin films with $16 \%$ of $\mathrm{Cu}$ atomic percentage. The resultant $\mathrm{CDZ}$ nano crystalline material was a mixture containing tetragonal and monoclinic phases. The $\mathrm{CuO}$ doped in the zirconia phases possesses very high red-ox reactivity $\left(\mathrm{Cu}^{2+} \leftrightarrow \mathrm{Cu}^{+}\right)$which is reversible at high temperature. The CDZ nano crystalline thin film was coated on the LPFG fiber device for high temperature sensing of $\mathrm{CO}$. $\mathrm{CO}$ detection by the CDZ-LPFG is realized through monitoring the shift of resonant wavelength $\left(\Delta \lambda_{R}\right)$ of the LPFG which was found to be a function of $\mathrm{CO}$ concentration. The CDZ-LPFG was demonstrated to be highly sensitive for CO detection at ppm-level concentration at $500-600^{\circ} \mathrm{C}$. The high temperature CO-sensing ability of the CDZLPFG relies on the CDZ refractive index variations caused by the reversible red-ox reactions when switching between the oxidizing (air) and reducing (CO) environments. However, it was also revealed by this study that the $16 \% \mathrm{Cu}$-doped $\mathrm{CDZ}$ was not stable under high-concentration of $\mathrm{CO}$ (e.g. 1vol\% tested here) due to the over reduction of $\mathrm{Cu}^{2+} / \mathrm{Cu}^{+}$to $\mathrm{Cu}^{0}$ that led to $\mathrm{Cu}$ segregation from the CDZ crystal structure and crystal structure changes in the CDZ solid. 


\subsection{Sensor performance enhancement by zeolite overcoate}

\subsubsection{Zeolite modification and characterization}

The resultant ZSM-5 film on the fiber surface was examined by field emission scanning electron microscopy (FESEM) and the actual elemental composition of the zeolite thin film was deter- mined by energy dispersive spectroscopy (EDS). Adsorption isotherms of $\mathrm{NH} 3, \mathrm{CO}$, and CH4 were measured by a Micro- meritics ASAP 2020 unit for the ZSM-5 zeolite particles collected from the residual liquid in the synthesis vessel. Both the zeolite- coated LPFG and ZSM-5 particles were treated in a $0.1 \mathrm{M} \mathrm{NH}_{4} \mathrm{Cl}$ solution at $80^{\circ} \mathrm{C}$ for 60 min after SDA removal to exchange the extra framework Na+ ions with NH4+. The ion exchanged ZSM-5 film and particles were then dried and calcined in air at $350^{\circ} \mathrm{C}$ to convert the $\left[\equiv \mathrm{Si}-\mathrm{O}^{-}\left(\mathrm{NH}^{+}{ }_{4}\right)\right]$ to $\left[\equiv \mathrm{Si}-\mathrm{O}^{-}\right.$ $\left.\left(\mathrm{H}^{+}\right)\right]$acidic sites in the zeolite structure.

\subsubsection{Morphology and chemistry of the zeolite film}

The zeolite film had a typical morphology of ZSM-5 films with low Si/Al ratios in which the individual particles do not have smooth surface and well-defined orientation planes. Although the outer surface of the ZSM-5 films is microscopically rough, a 3-4 mm thick dense layer is clearly formed at the fiber surface. This film thickness ensures that the cladding modes are confined in the cladding so that the influence of film thickness variation on $1 \mathrm{R}$ is negligible for different sensors.

The EDS test confirmed that both the zeolite film and particles collected from residual liquid had similar average $\mathrm{Si} / \mathrm{Al}$ ratios of $22.8(1.5)$ and a $\mathrm{Na}^{+} / \mathrm{Al}^{3+}$ atomic ratios of $\sim 0.74(0.1)$. The $\mathrm{Na}^{+} / \mathrm{Al}^{3+}$ atomic ratio in the zeolite decreased to $\sim 0.1$ after the $\mathrm{NH}^{4+}$ ion exchange and firing. The 
MFI zeolite unit cell composition is $\mathrm{M}^{\mathrm{m}+}\left[\mathrm{Si}_{96-\mathrm{x}} \mathrm{Al}_{\mathrm{x}} \mathrm{O}_{192}\right]$ where $\mathrm{M}^{\mathrm{m}+}$ is the extra- $\mathrm{x} / \mathrm{m}$ 96 x x 192 framework cation compensators, which are $\mathrm{Na}^{+}$and $\mathrm{H}^{+}$in the current ZSM-5 zeolites. Thus, in each unit cell, the as-synthesized ZSM-5 contained $\sim 3.0\left[\equiv \mathrm{Si}_{-}^{-} \mathrm{O}^{-}\left(\mathrm{Na}^{+}\right)\right]$sites and $\sim 1.0\left[\equiv \mathrm{Si}-\mathrm{O}^{-}\right.$ $\left.\left(\mathrm{H}^{+}\right)\right]$site while the modified HZSM-5 contained $\sim 0.4\left[\equiv \mathrm{Si}-\mathrm{O}^{-}\left(\mathrm{Na}^{+}\right)\right]$sites and $\sim 3.6\left[\equiv \mathrm{Si}-\mathrm{O}^{-}\left(\mathrm{H}^{+}\right)\right]$ sites. The modified ZSM-5 film on LPFG and ZSM-5 zeolite particles had increased numbers of acidic sites and hence is denoted hereafter as HZ-LPFG and HZSM-5, respectively.

\subsubsection{Optical gas sensing}

The transmission spectrum and $1 \mathrm{R}$ of the Z-LPFG were measured by an apparatus same as that reported in previous works. The zeolite-coated LPFG segment of the fiber was mounted in a 4-mm- ID stainless steel tube (total chamber volume $\sim 9.6 \mathrm{~cm}^{3}$ ) which was placed in a temperature-programmable tubular furnace. The transmission spectrum covers a near IR wavelength range of $1510-1640 \mathrm{~nm}$. The source light was provided by a tunable laser equipped with a laser power detector (Agilent 8164A), and a computer data acquisition system. In operation, one end of the Z-LPFG connects to the tunable laser (light source) and the other end (output) connects to the laser power meter. The output light intensity was measured point-bypoint by the power meter to obtain the transmission spectrum and the $1 \mathrm{R}$ value. A wavelength increment of $1 \mathrm{~nm}$ and a dwelling time of $1 \mathrm{~s}$ were used in the gas sensing experiments. In all measurements, the total flow rate of the sample gas was kept at a small value of $10 \mathrm{~cm}^{3}$ (STP)/min for maintaining temperature stability and avoiding mechanical disturbance to the suspended fiber sensor.

\subsubsection{Effect of temperature on sensor performance}

The temperature-dependence of gas adsorption in zeolites varies with the adsorbing strength. Adsorption isotherms were measured for $\mathrm{NH}_{3}$ and $\mathrm{CO}_{2}$ in a temperature range of 35 to $200^{\circ} \mathrm{C}$. 
Distinct effects of zeolite modification were obtained on the adsorption of $\mathrm{NH}_{3}$ and $\mathrm{CO}_{2}$. At low temperature, physical adsorptions of $\mathrm{NH}_{3}$ is significant in both the ZSM-5 and HZSM- 5 zeolites and the total amounts of $\mathrm{NH}_{3}$ adsorption in ZSM-5 and HZSM-5 are almost the same. As temperature increases, the $\mathrm{NH}_{3}$ physisorption diminishes and the $\mathrm{NH}_{3}$ chemisorption remains strong. Hence the HZSM-5 zeolite adsorbs a larger amount of $\mathrm{NH}_{3}$ than the ZSM-5 zeolite at elevated temperature. In contrast, the extra frame work alkali metal ions enhance the adsorption of $\mathrm{CO}_{2}$ gas. Thus, replacing the $\mathrm{Na}+$ with $\mathrm{H}+$ in the zeolite reduces the adsorption of the acidic $\mathrm{CO}_{2}$. The relatively weak adsorption of $\mathrm{CO} 2$ diminished to nearly zero at $110-150^{\circ} \mathrm{C}$ in the HZSM-5 and at $\sim 200^{\circ} \mathrm{C}$ in the ZSM-5 zeolite, respectively. Meanwhile, the chemisorption of NH3 remained significant in both zeolites at $200^{\circ} \mathrm{C}$ namely $13.4 \mathrm{~cm}^{3}(\mathrm{STP}) / \mathrm{g}$ in ZSM-5 and $18.32 \mathrm{~cm}^{3}(\mathrm{STP}) / \mathrm{g}$ in HZSM-5.

Therefore, the modification of zeolite enhances the $\mathrm{NH}_{3}$ chemisorption while reducing the $\mathrm{CO}_{2}$ adsorption that, in combination, dramatically increased the $\mathrm{B} \mathrm{NH}_{3} / \mathrm{CO}_{2}$ in the HZSM-5, especially at elevated temperatures. The gas adsorption data indicate that the sensing selectivity for $\mathrm{NH}_{3}$ over $\mathrm{CO}_{2}$ can be significantly improved by the surface modification together with proper selection of operation temperature.

The above results demonstrate that the $\mathrm{NH}_{3}$ sensing selectivity by the HZ-LPFG can be greatly improved when operating at elevated temperatures. The operating temperature will also affect the speed of response and the detection sensitivity. The speed of response in gas sensing is expected to be faster at higher temperature because the endothermic desorption as well as diffusion of $\mathrm{NH}_{3}$ molecules in the micro-porous zeolite are facilitated at high temperature. Meanwhile, the $\mathrm{NH}_{3}$ adsorption in the zeolite is reduced at elevated temperature that decreases the magnitude of $\mathrm{DlR}, \mathrm{NH}_{3}$ to lower the sensitivity. 
Single wavelength sensing experiments were performed to investigate the HZ-LPFG temporal response to switching sample gas flow between pure $\mathrm{N}_{2}$ and a mixture of $612 \mathrm{ppmv}$ $\mathrm{NH}_{3}$ carried in $\mathrm{N}_{2}$. The dynamic response of transmission light intensity to the switch of gas composition was monitored at a fixed wavelength. The fixed single wavelength was specifically selected for each temperature such that the variation of the transmission light intensity would be the same if same amount of DIR occurs.

ZSM-5 zeolite films with a low Si/Al ratio of $23 \%$ have been synthesized on LPFG optical fibers. The zeolite-coated LPFG (Z-LPFG) offers high sensitivity for gas sensing by monitoring the shift of the resonant wavelength upon molecular adsorption in the zeolite. The sensing selectivity of the Z-LPFG relies on the selectivity of gas adsorption in the zeolite pores. Zeolite surface modification combined with temperature control provides an effective way to enhance the gas sensing selectivity and response speed. However, operation at high temperature compromises the sensitivity. While the sensing performance in multi-component mixtures is yet to be investigated, this study shows the feasibility of using zeolites as effective optical chemical sensing materials for construction of fiber optic sensors. The present work demonstrates that the optical response of HZ-LPFG to $\mathrm{NH}_{3}\left(\mathrm{DIR}, \mathrm{NH}_{3}\right)$ has excellent quantitative correlations with $\mathrm{NH}_{3}$ concentration and temperature. Thus with temperature and concentration $\left(\mathrm{CNH}_{3}\right)$ calibration data, a mathematic model of $\mathrm{DlR}, \mathrm{NH}_{3} 1 / 4 \mathrm{f}\left(\mathrm{T}, \mathrm{CNH}_{3}\right)$ can be readily established and built into the computer program for quantitative measurement. There exist a large number of zeolitic materials with distinct crystallographic structures, pore sizes, material chemistry, and capacities for surface modification. This offers an excellent opportunity to develop a new class of zeolite-enabled fiber optic chemical sensors for applications in many important areas such as energy production processes, environmental monitoring, and chemical and biological threat 
detection. The zeolite-fiber integrated device is also potentially useful for studying molecular sorption and diffusion mechanisms in zeolites because of its high sensitivity and temporal resolution. 


\subsection{Sensor installation and testing in an experimental gasifier}

\section{$\underline{\text { 3.5.1 Introduction }}$}

Encouraged by the results in fundamental research, the research team has been tasked to move a step further into the applied research of testing the developed sensors in a university experimental gasifier. In this new task, we have conducted research in packaging of the sensors, modification of the gasifier for sensor installation, and development of instrumentation for onsite data logging.

\subsubsection{Material testing for sensor packaging}

Placement of the long period fiber grating (LPFG) sensor inside the exhaust pipe for detection of syngas is an important task. Bare fiber cannot survive very high temperatures and pressure inside the exhaust pipe. For the sensor to work in high temperature and high pressure environment, a highly effective packaging is needed that will allow the sensing devices to work the same way as they would without the packaging. The purpose of the packaging is to provide a protective shield to the sensing device from high temperature and pressure. To successfully instal the sensor and for protecting it from high temperature and pressure environment, variety of material were tested. The material/products tested include regular tin-lead $(\mathrm{Sn}-\mathrm{Pb})$ solder, silver solder, and a ceramic adhesive (RESBOND (940 LE).

Stainless steel tube ( $(1 / 8$ " Swagelok tube) was used as the casing to encase the fiber sensor inside it. Fiber was gold coated, using the process of sputtering, for it to operate in high temperature ambient environment. Above listed products were then used to position the fiber inside the tube. 
Because of the lower temperature requirements, it was easier to achieve the molten state of $\mathrm{Sn}-\mathrm{Pb}$ solder and place it inside the tube at a desired position. A regular solder gun was used for melting and application of the solder inside the tube. But the sharp tip of the soldering gun makes the fiber vulnerable to cuts. Soldering inside the tube around the inner circumference was achieved easily but soldering around the fiber was difficult. Silver solder however was not easy to melt. A welding torch was used to melt the solder and the molten state was achieved but silver solidifies very quickly and thus could not be filled inside the tube. Brazing the silver solder with the tube was tried in a tube furnace. But its proper positioning around the fiber could not be achieved. Ceramic adhesive was most easy to work with in terms of its preparation and application inside the tube. A mixture of 100 parts of ceramic adhesive with 45 parts of a compatible agent was used as glue to make the fiber stay in place. Ceramic adhesive comes in a powder form which is an easy to use product.

\section{Experimental Results}

The most successful among the tried products was the ceramic adhesive. It can sustain very high temperatures of up to $1370^{\circ} \mathrm{C}$. It does not melt at high temperatures once properly cured. It takes 24 hours for proper curing. The application of the mixture (ceramic adhesive and the compatible agent) was also easy since its in gel like state initially and can be inserted into the tube very easily. After filling the tube to gain desired thickness of the adhesive, fiber was inserted through the mixture and the sample was left for 24 hours for the glue to settle. It was tested for temperature of up to $650^{\circ} \mathrm{C}$ successfully inside a tube furnace. Table 5 shows one of the data sets showing the state of the material at a particular temperature. Test time only reflects the time at one particular temperature and the total time reflects the total time the sample has 
been exposed to the specified temperature or less.

Table $5 *$ Temperature testing using the ceramic adhesive

\begin{tabular}{|c|l|l|l|}
\hline Temperature & Test Time & \multicolumn{1}{|c|}{ Total Time } & \multicolumn{1}{|c|}{ Result } \\
\hline $200^{\circ} \mathrm{C}$ & 15 minutes & 15 minutes & $\begin{array}{l}\text { No signs of deterioration in the } \\
\text { material. }\end{array}$ \\
\hline $400^{\circ} \mathrm{C}$ & 15 minutes & 30 minutes & $\begin{array}{l}\text { No signs of deterioration in the } \\
\text { material. }\end{array}$ \\
\hline $500^{\circ} \mathrm{C}$ & 15 minutes & 45 minutes & $\begin{array}{l}\text { No signs of deterioration in the } \\
\text { material. }\end{array}$ \\
\hline $650^{\circ} \mathrm{C}$ & 30 minutes & 75 minutes & $\begin{array}{l}\text { No signs of deterioration in the } \\
\text { material. }\end{array}$ \\
\hline
\end{tabular}

* Transition time that the furnace took to make transition from a lower temperature to a higher temperature is not included in the data summarized in table 1 and table 3 . Transition times would easily add up to at least 30 minutes for Ceramic adhesive. Transition time varied from about 5 minutes to about 15 minutes depending upon the target temperature.

Table 6 shown below presents the data for pressure testing of the ceramic adhesive sample. It was tested against the pressures of up to 500 psi. The sample tested very well against high pressure. No leaks were encountered in the sample. 
Table 6 Pressure testing using the ceramic adhesive

\begin{tabular}{|c|l|}
\hline Test Pressure & Result \\
\hline $100 \mathrm{psi}$ & No leaks in the sample. \\
\hline $300 \mathrm{psi}$ & No leaks in the sample. \\
\hline $500 \mathrm{psi}$ & No leaks in the sample. \\
\hline
\end{tabular}

Regular tin-lead solder was tested for temperature and pressure in the same way as the ceramic adhesive. Results obtained from temperature/pressure testing of the solder are tabulated in Table 7 and Table 8 respectively. At temperatures of about $250^{\circ} \mathrm{C}$, the solder started vaporizing and almost one-third of the total layer was gone. Pressure testing of the solder did not show any promising results either. There were no leaks when it was tested against 100 psi of pressure but it did not tested well for pressures above 100 psi.

Table 7 Temperature testing using regular $\mathrm{Sn}-\mathrm{Pb}$ solder

\begin{tabular}{|c|c|l|}
\hline Temperature & Test Time & Result \\
\hline $100^{\circ} \mathrm{C}$ & 10 minutes & No signs of melting. \\
\hline $200^{\circ} \mathrm{C}$ & 15 minutes & Some signs of melting. \\
\hline $250^{\circ} \mathrm{C}$ & 15 minutes & $\begin{array}{l}\text { About one-third of the thickness had } \\
\text { diminished/deteriorated. }\end{array}$ \\
\hline
\end{tabular}


Table 8 Pressure testing using regular $\mathrm{Sn}-\mathrm{Pb}$ solder

\begin{tabular}{|c|l|}
\hline Test Pressure & Result \\
\hline $100 \mathrm{psi}$ & Held find against this pressure. \\
\hline$>100 \mathrm{psi}$ & Several leaks in the sample. \\
\hline
\end{tabular}

\section{Conclusion}

Ceramic adhesive provided with the best results against high temperature and high pressure both. It was very easy to work with and due to its initial gel like state, poses no danger to the fiber as well.

\subsubsection{Design modifications in the gasifier exhaust pipe}

The placement and installation of the LPFG sensor inside the exhaust pipe is very important. Proper installation of the sensor would ensure proper gas detection and efficient overall system functionality. Figure 96 below shows the arrangement of the LPFG sensor placement.

This design takes into account the maximum exposure of LPFG sensor to the exhaust pipe environment for better detection of the syngas. Micro-vents will be made into the Swagelok tube (across the section of the fiber containing the grating) for maximum exposure the ambient environment. Swagelok connectors and reduction connectors will be used to connect shorter section of the tubes that are not uniform in shape or size to handle the fiber properly. A loop type structure has been incorporated in order to minimize any installation related strain on the fiber. As shown in the Figure, the fiber will only be fixed at one point to provide maximum 
flexibility. The modifications in the design have been communicated to Innovator Inc. for realizing the changes in the actual set-up.

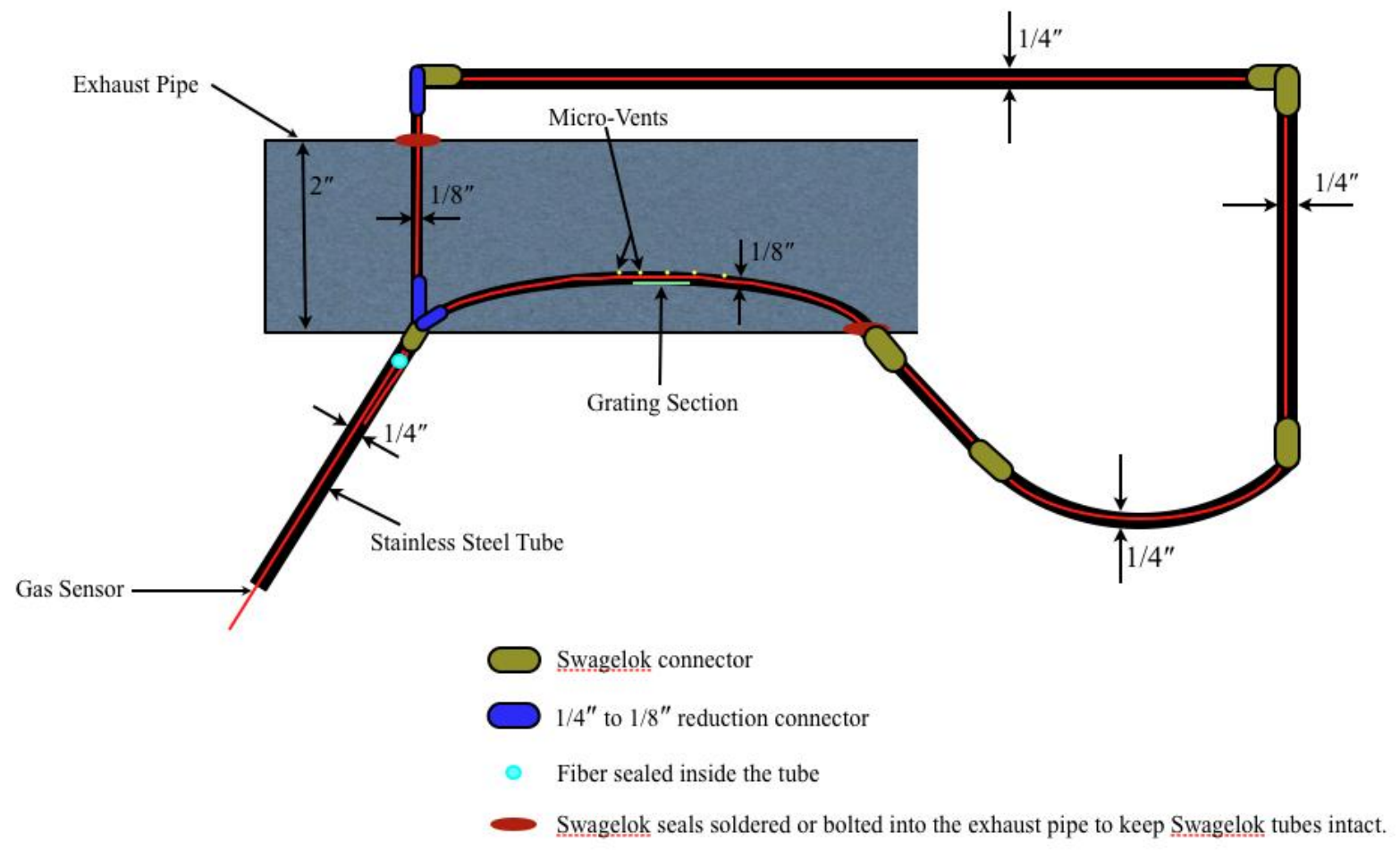

Figure 96 Schematic showing the LPFG sensor placement arrangement

\subsubsection{Design and Installation of Gas Chamber for Sensor Installation}

A chamber for the installation of Optical fiber sensor was designed together with Innoventor, Inc. at St. Louis. Various reviews were carried out to meet the designing requirements and make it feasible for manufacturing. Figure 97 shows the actual product manufactured by Innoventor, inc. The chamber is designed such that the sensor can be installed perpendicular to the gas flow in order to provide maximum exposure. The sensor is packaged inside a stainless steel swagelok tube. Minute slits are made through the tube across the length of the sensor for providing an inlet 
for gases and to make detection of the ambient environment accessible. Multiple ports are provided for installing more than one sensor at a time for multiple parameter detection.

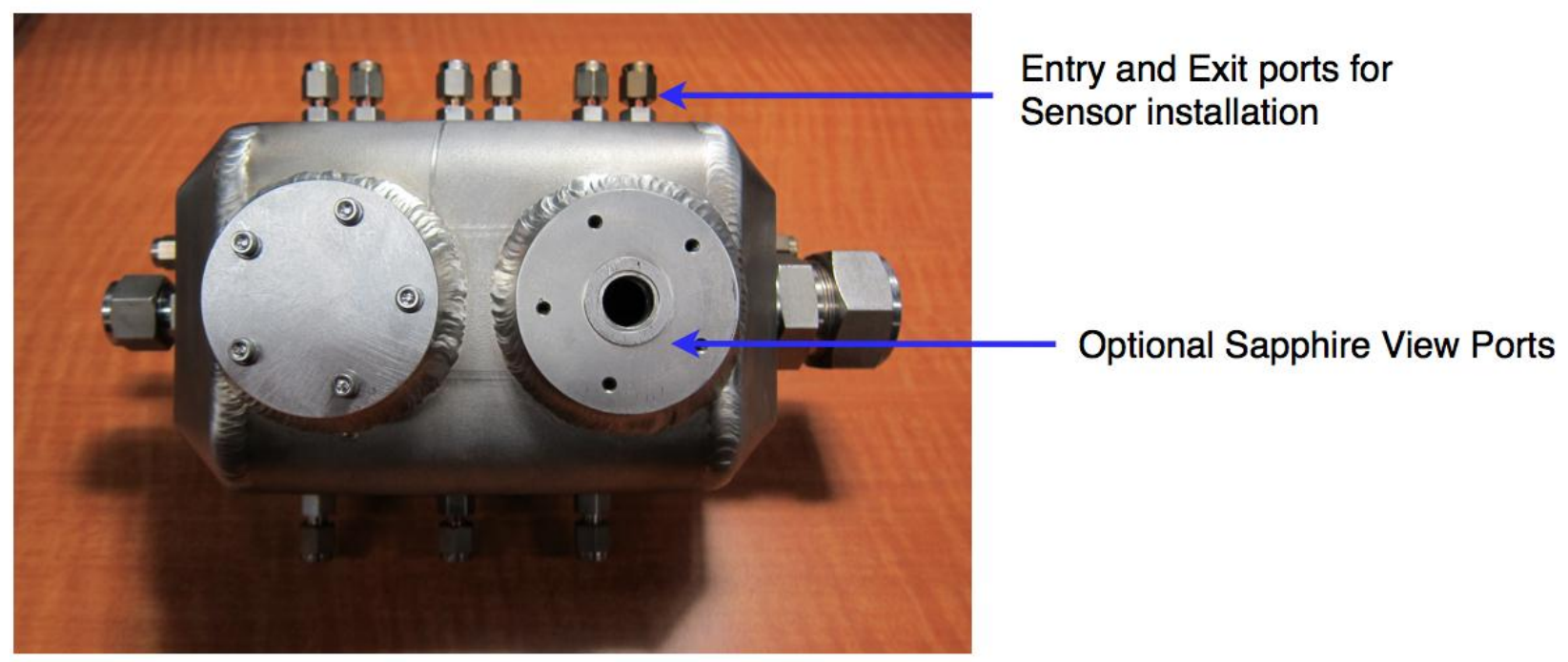

Figure 97 Chamber installation and testing, installed chamber is shown in a red circle

As shown in the Figure above, there is a provision for two view ports for in-situ monitoring of the inside environment. Sapphire see-through sheet can be installed if required for looking inside the chamber. The chamber is designed to withstand a temperature of up to $600^{\circ} \mathrm{C}$ and pressure of up to 200psi continually for hours. A stand rig is used to install the chamber at the same height as the exhaust pipe for optimal connection and feed of gas through the chamber. An inline-heater and temperature controller will be used for maintaining the temperature of the gas exiting the gasifier preventing extreme differences in the temperature inside and outside the chamber. 


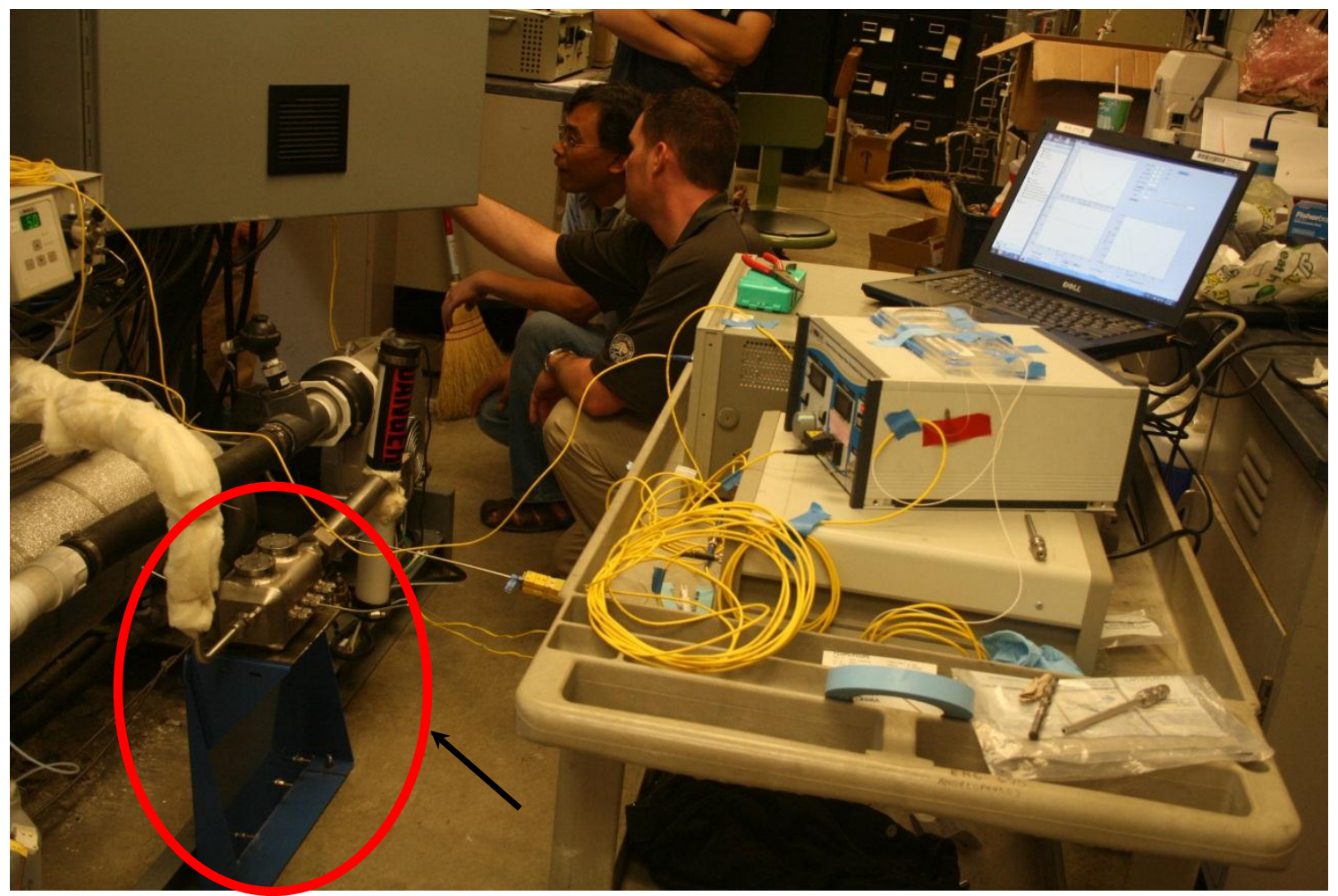

Figure 98 Chamber installation and testing, installed chamber is shown in a red circle.

The chamber for sensor installation has been tested for desired pressure and temperature levels. It was recently installed at University of Cincinnati for further testing. Sensor installation within the chamber was also tested. The chamber was designed taking into consideration that there should be minimum installation induced stress on the optical fiber sensor. The sensor was only fixed at a single entry/exit point to minimize the tension so that signal to noise ratio could be higher. Figure 99 shows the testing procedure going on for testing the chamber for high temperature and high pressure. 


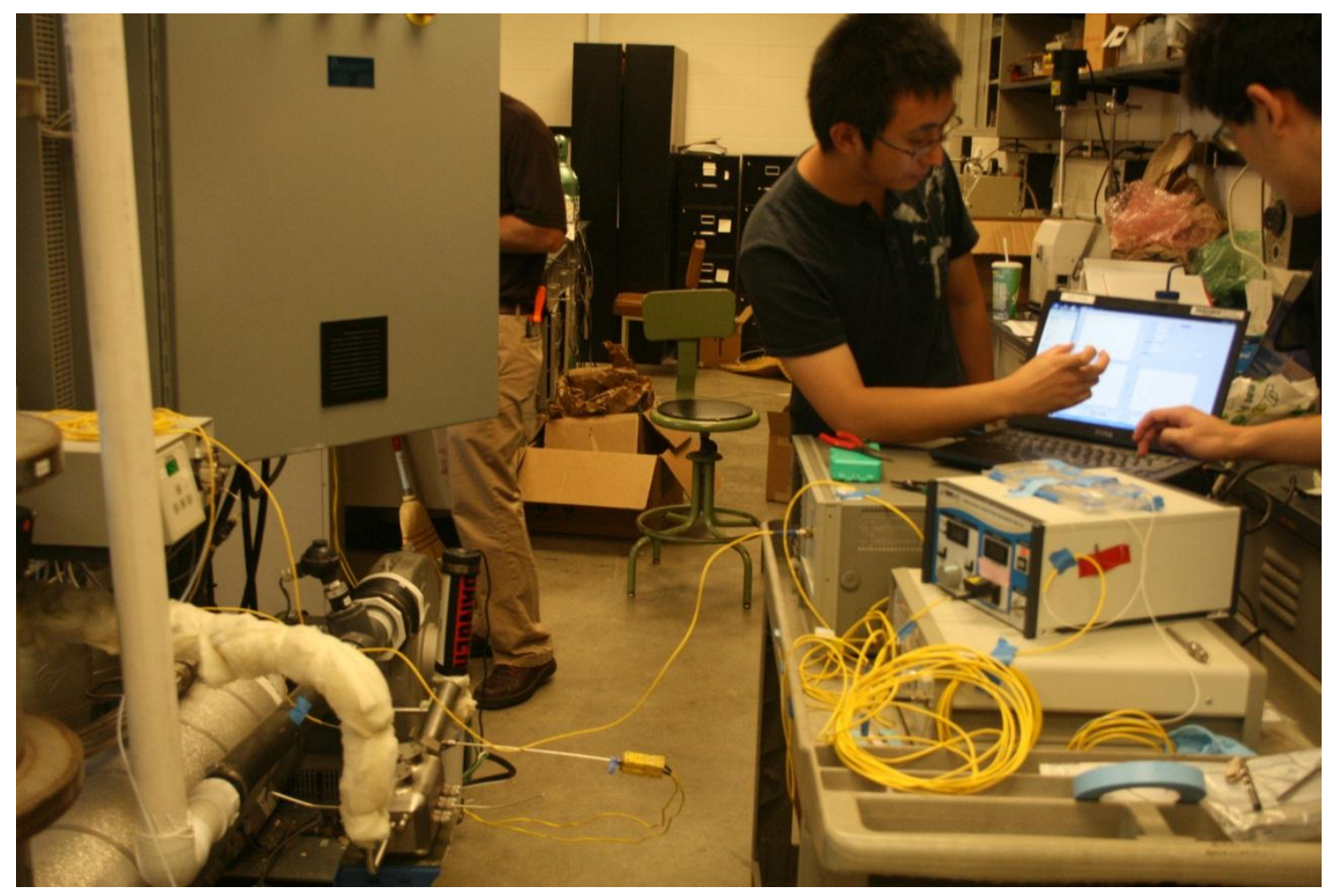

Figure 99 Picture of field testing

The gasifier has been modified with the sensor test chamber built in the gasifier's exitpipeline where the syngas temperature is around $500^{\circ} \mathrm{C}$. The sensor hosting chamber has two peek windows and eight sensor installation ports one of which is in parallel to the gas flow (for future online GC-MS measurement as sensor validation). The sensor is packaged inside a stainless steel tube $\left(1 / 8^{\prime \prime}\right.$ o.d.) with small pore openings $(\phi-1 \mathrm{~mm})$ distributed over the length of the sensing segment $(\sim 5 \mathrm{~cm})$ to allow for direct and effective contact with the syngas flow. An inline gas heater is mounted before the sensor chamber for controlled temperature variation for sensor evaluation purpose. The SCZY-LPFG sensor is oriented perpendicular to the gas flow direction together with a fiber optic temperature sensor (LPFG) for simultaneous temperature monitoring which is required for temperature compensation in gas composition measurement. A thermocouple is installed near the fiber sensors for temperature verification. Figure 100 shows 
the actual sensor equipped gasifier system.

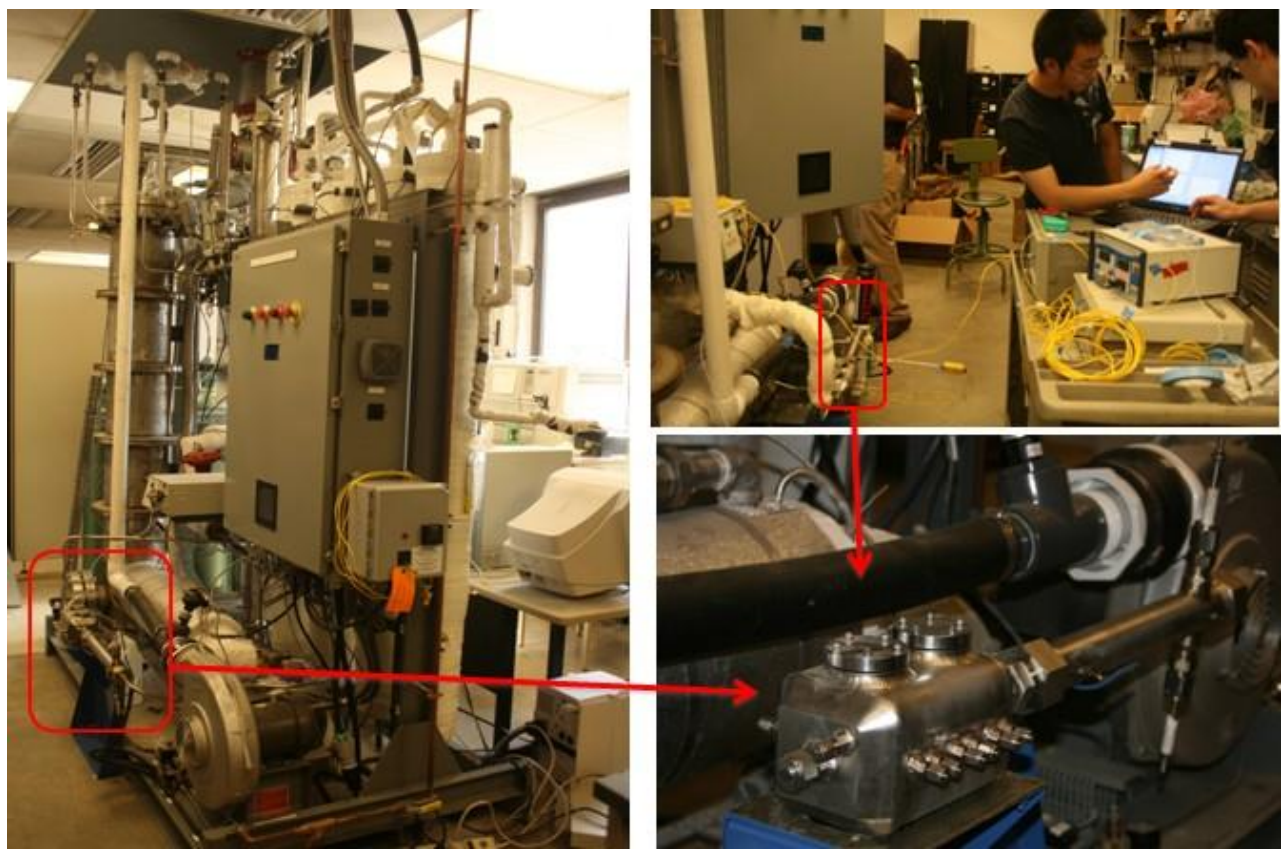

Figure 100 Pictures for the actual gasifier equipped with the sensor test unit.

\subsubsection{Development of instrumentation for on-site data logging}

Once the sensor installation is complete and the system is ready to be tested, data acquisition will be an important part of the system performance analysis. A remote log-in system will be used to prompt the initiation of data collection and also to stop the process.

The methodology and overview of the system to be used for data acquisition is shown below. Two computers (one present at the location of the data acquisition and the other at Missouri S\&T location for remote access) equipped with WebEx PCnow, NI controller, and MATLAB 2010b will be used. WebEx provides features like remote login, and screen or desktop sharing between compatible computers. After remotely logging into the system, a MATLAB graphical user interface (GUI) will be prompted to recognize and read General Purpose Interface 
Bus card (GPIB). Then to read and acquire the data from the Optical Spectrum Analyzer (OSA), commands will be sent through the GUI. This data will be stored in a folder (made in MATLAB itself) that will be accessible remotely for downloading and processing the data.

\section{Remote Log-in using WebEx}

Prompt GUI to recognize and read GPIB card

Send OSA commands to read and acquire the data

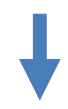

Store data in an accessible folder

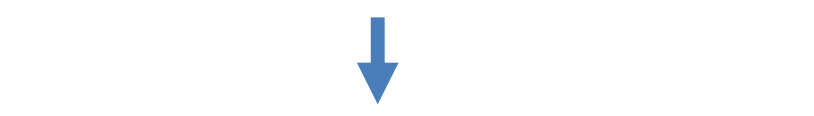

Download the data

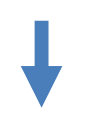

Process and analyze the data

Instrumentation for sensor data acquisition and interrogation has been completed. The system includes a white-light source made by multiplexing a conventional band (C-band, AFC BBS1550A-TS) and a long band (L-band, Highwave HWT-BS-L-P) Erbium-doped fiber (EDF) 
amplified spontaneous emission (ASE) sources together. The OSA is a HP 70952B made by Agilent. The system has been integrated and a fiber coupler/circulator circuit has been implemented for convenient sensor connection. Fully automated control of the instruments has been realized by Visual Basic programming. The system is now capable of perform fully automated sensor interrogation through remote log-on.

Both the gasifier and the sensor monitoring systems are fully controlled by computer programs (Figure 101 a and b). The Optical Spectrum Analyzer (OSA) is connected to an optical switch which has the capacity for simultaneous data acquisition from eight fiber sensors.
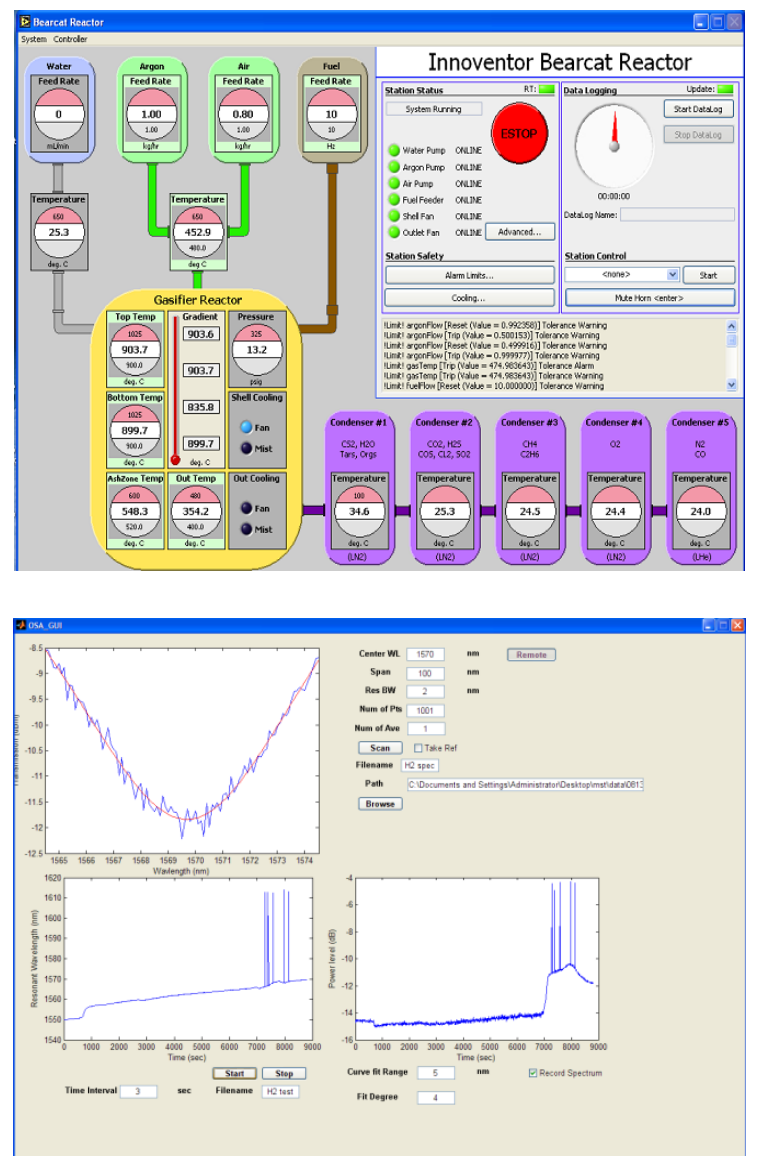

Figure 101 Screenshots of the gasifer (top) and sensor (bottom) computer operating programs. 


\section{$\underline{\text { 3.5.6 Test of } \mathrm{H}_{2}-\text { Sensor for In-Situ Monitoring of Coal Gasification Syngas }}$}

The senor tests were conducted over short time gasifier operation cycles with each cycle included temperature ramping under inert gas flow (Helium), coal feed, and dilute air feed, coalfeed termination, and cooling (shutdown). Table 9 give the gasification conditions of two separate tests and Table 10 presents the results of $\mathrm{H}_{2}$ molar fractions obtained by the SCZYLPFG sensor and GC analysis. The SCZY sensor tested has a temperature response of 0.105 $\mathrm{nm} /{ }^{\circ} \mathrm{C}$ and $\mathrm{H}_{2}$ sensitivity of $0.1830 \mathrm{~nm} / \mathrm{kPa}-\mathrm{H}_{2}$ at $500^{\circ} \mathrm{C}$. The Ohio coal used had a composition of $45 \%$ carbon, $\sim 10 \%$ sulfur, and $\sim 5 \%$ moisture. As shown in Table 10, the $\mathrm{H}_{2}$ concentrations in the syngas obtained by the sensor and GC analyses are in reasonable agreement. The differences in $\mathrm{H}_{2}$ concentration measurement between the sensor and the GC are likely caused by (1) relatively large error in temperature measurement by the accompanying fiber temperature sensor which was not calibrated in-situ and (2) the error involved in GC sampling because currently online GC-MS is not installed.

Table 9 Gasification conditions for two separate sensor tests

\begin{tabular}{|c|c|}
\hline Test No. 1 & Test No. 2 \\
\hline Gasification conditions: & Gasification conditions: \\
Gas: $56 \%$ Argon $+44 \%$ air & Gas: $56 \%$ Argon $+44 \%$ air \\
Total gas feed flow rate: $1.8 \mathrm{~kg} / \mathrm{h}$ & Total gas feed flow rate: $1.5 \mathrm{~kg} / \mathrm{h}$ \\
Coal feed rate: $1 \mathrm{~kg} / \mathrm{h}$ & Coal feed rate: $0.42 \mathrm{~kg} / \mathrm{h}$ \\
Temperature: $905 \sim 915{ }^{\circ} \mathrm{C}$ & Temperature: $905 \sim 915{ }^{\circ} \mathrm{C}$ \\
Pressure: 27.7 Psi & Pressure: 27.7 Psi \\
Sensor temperature: $503{ }^{\circ} \mathrm{C}$ & Sensor temperature: $494{ }^{\circ} \mathrm{C}$ \\
& \\
\hline
\end{tabular}


Table 10 Comparison of $\mathrm{H}_{2}$ composition by fiber sensor and $\mathrm{GC}$ analysis $(\mathrm{T}=500 \mathrm{oC}$ and $\mathrm{P}=27.7$ Psi)

\begin{tabular}{|c|c|c|c|}
\hline \multicolumn{2}{|c|}{ Test } & $\begin{array}{c}\text { Sampling } \\
\text { No. }\end{array}$ & SCZY \\
Points & sensor & \\
\hline 1 & 5 & $11.8 \%$ & $8.9 \%$ \\
\hline 2 & 7 & $7.4 \%$ & $6.0 \%$ \\
\hline
\end{tabular}

\section{Sensor Data Processing}

Figure 102 shows an example of temperature and $\mathrm{H}_{2}$ fiber sensor spectra evolution during the gasification monitoring process. Figure $\mathbf{1 0 3}$ shows the online recorded raw data of SCZYLPFG resonant wavelength $\left(\Delta \lambda_{R}\right)$ changes during the $\sim 50$ min gasifier operation together with simultaneously recorded temperature changes. Figure 104 presents the separated $\Delta \lambda_{\mathrm{R}}$ changes caused by $\mathrm{H}_{2}$ concentration and temperature variations based on the SCZY-LPFG's temperature dependence and $\mathrm{H}_{2}$ composition (partial pressure dependence). Figure 105 gives the sensor detected $\mathrm{H}_{2}$ composition in the gasifier syngas stream in terms of $\mathrm{H}_{2}$ partial pressure and mole fraction. 


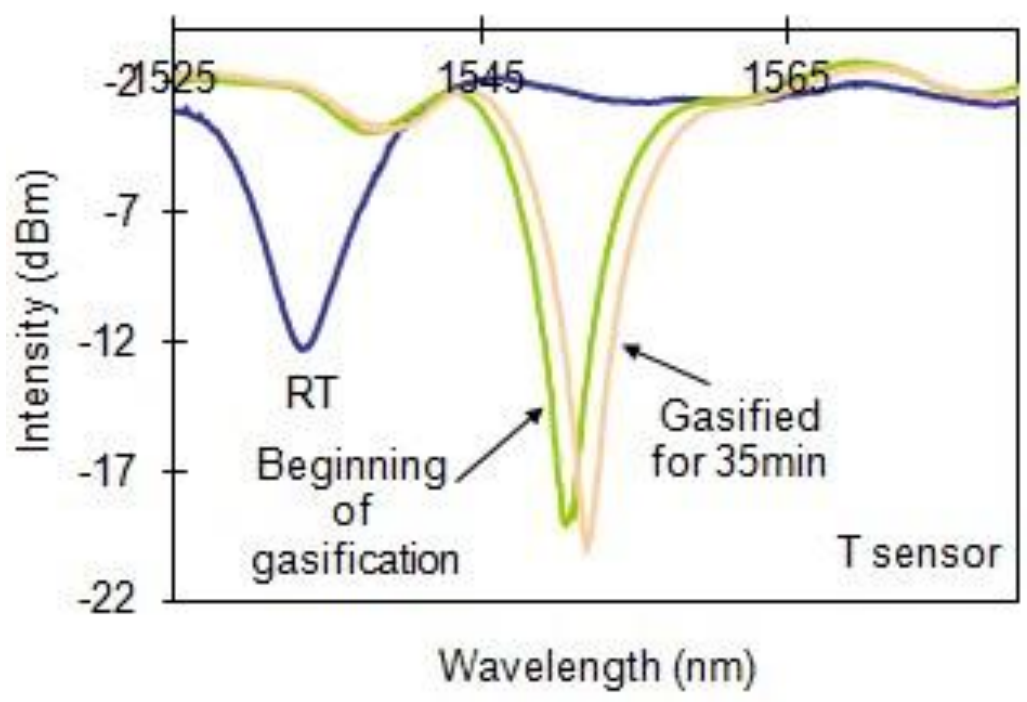

(a) Transmission spectra of the temperature sensor during tests

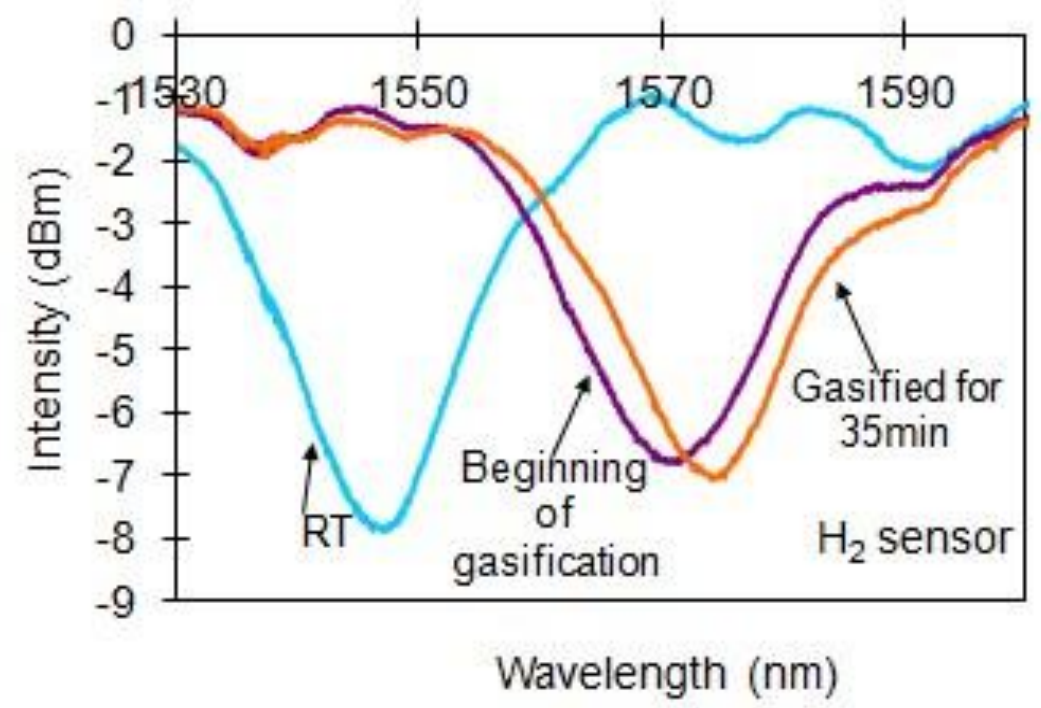

(b) Transmission spectra of the hydrogen sensor during tests

Figure 102 Transmission spectra shifts for the temperature sensor (LPFG) and the $\mathrm{H}_{2}$ sensor (SCZYLPFG) at various stages of gasifier operation (in Test No. 2) 


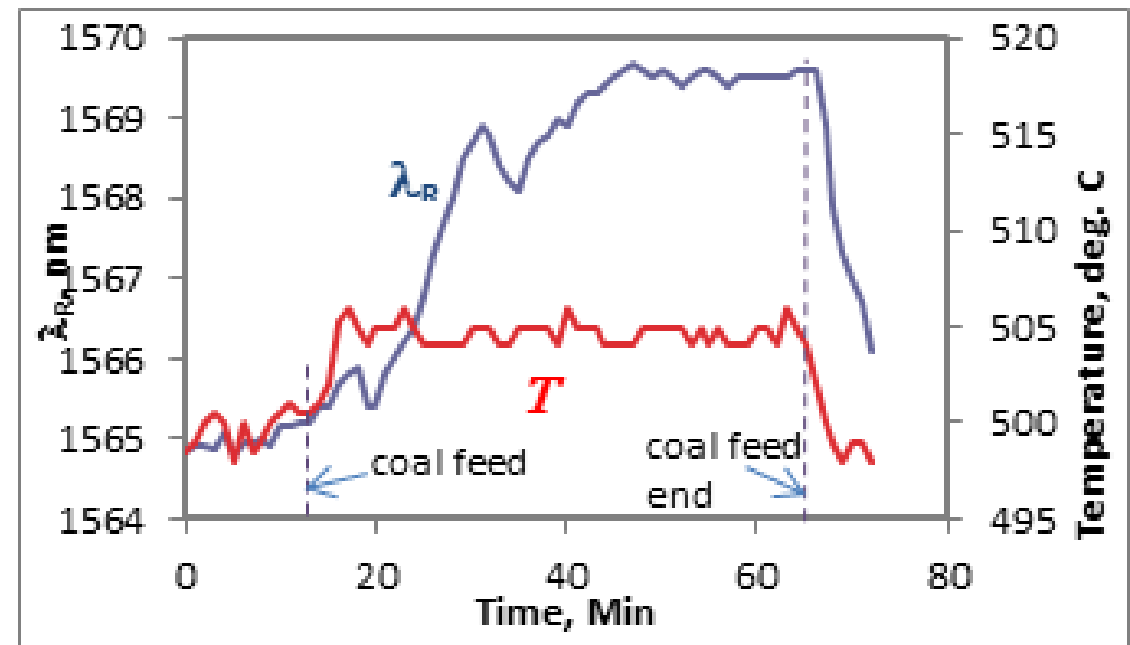

Figure 103 Online measured SCZY-LPFG resonant wavelength $\left(\lambda_{R}\right)$ and temperature as function of time during gasification:

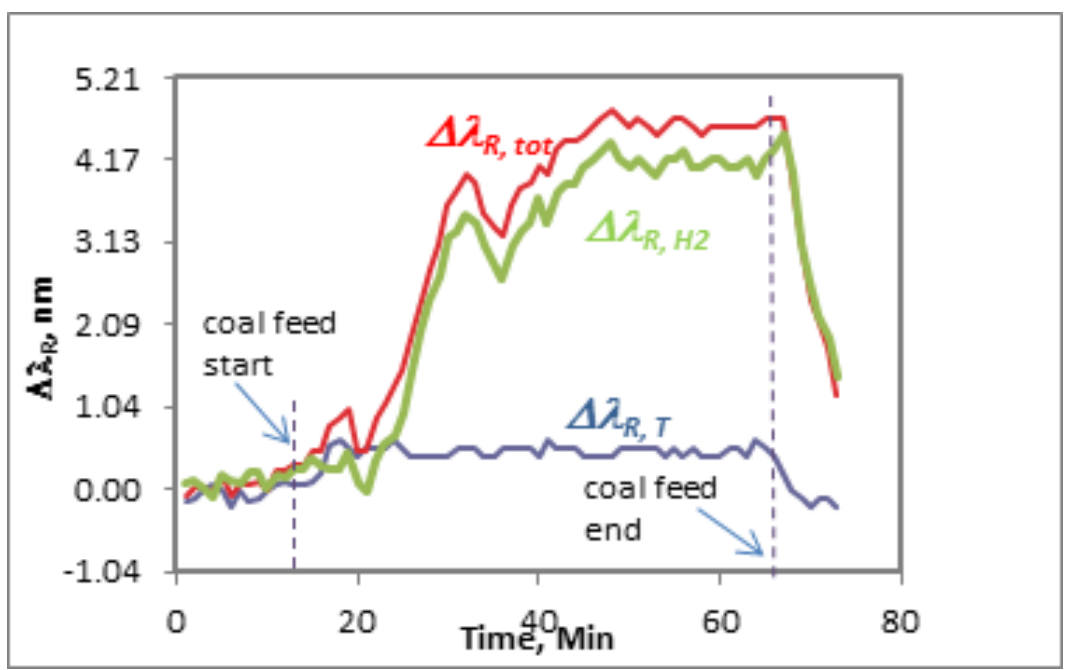

Figure 104 Wavelength shifts $\left(\Delta \lambda_{R}\right)$ of the SCZY-LPFG caused by temperature change and $\mathrm{H}_{2}$ concentration (partial pressure) change as function of operation time (online monitoring). 


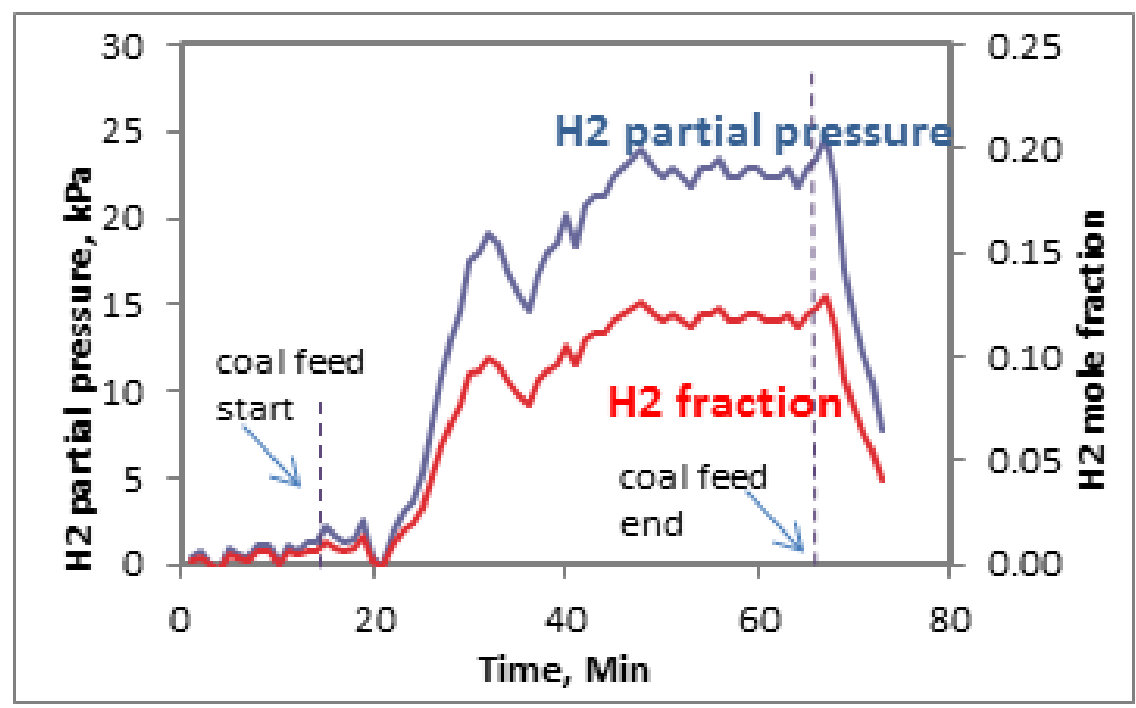

Figure 105 Hydrogen content monitored during gasification (partial pressure vs. time; and mole fraction vs. time)

\section{Conclusions}

In summary, this has been a truly successful project. The research team has completed all the technical milestones on time and within budget. The major research accomplishments are summarized below:

1) Developed a stable fiber gas sensor platform -nanocrystalline doped ceramic coated LPFG for gas sensing in high temperature and high pressure harsh environments. The sensors have been extensively studied, fabricated, characterized and demonstrated for sensing applications.

2) Identified various doped ceramic materials for optical gas sensing. The materials have been extensively studied, characterized for gas sensing in harsh environments. 
3) In addition to LPFG, other miniaturized fiber devices have also been explored and researched for harsh environment sensing. These new fiber sensor devices would contribute to future sensor development.

4) Developed methods for processing and coating nanocrystalline doped ceramic sensing materials onto LPFGs. The developed ceramic-doped sensors have been successfully demonstrated with good performance for gas sensing applications.

5) Implemented sensor instrumentation to support the sensor development, test and evaluations. The sensor instrumentation has been proven to be robust and effective to support the success of the various research tasks.

6) Demonstrated the feasibility of developed sensors in laboratory simulated conditions. The sensors have shown excellent sensitivity, good long term stability and selectivity for gas sensing.

7) Demonstrated the feasibility of the developed sensor in an experimental gasifier at the University of Cincinnati. The sensors have been successfully packaged and installed into the gasifier. The developed sensors have been successfully survived the harsh conditions and operated for a long-period time. The $\mathrm{H}_{2}$ concentrations in the syngas obtained by the sensor and GC analyses are in a very good agreement. 


\section{Journal Publications}

1. X. Lan, Q. Han, T. Wei, J. Huang, H. Xiao, "Turn-Around-Point Long-Period Fiber Gratings Fabricated by CO Laser Point-by-Point Irradiations," IEEE Photonics Technology Letters, vol. 23, pp. 1664-1666, 2011.

2. C.L. Eggen, Y.S. Lin, T. Wei, H Xiao, "Detection of Lipid Bilayer Membranes Formed on Silica Fibers by Double-Long Period Fiber Grating Laser Refractometry," Sensors \& Actuators: B. Chemical, v.150, pp.734-741, 2010.

3. Y. Han, T. Wei, H.L. Tsai, H. Xiao, "Measurement of refractive index change of optical fiber core induced by femtosecond laser scanning,” Optical Engineering, vol.49, pp.064301, 2010.

4. Y. Zhang, Y. Li, T. Wei, X. Lan, Y. Huang, G. Chen, H. Xiao, "Fringe Visibility Enhanced Extrinsic Fabry-Perot Interferometer Using a Graded Index Fiber Collimator," IEEE Photonics Journal, vol.2, pp.469-481, 2010.

5. X. Wei, T. Wei, J. Li, X. Lan, H. Xiao and Y.S. Lin, "Strontium Cobaltite Coated Optical Sensors for High Temperature Carbon Dioxide Detection," Sensors \& Actuators: B. Chemical, V.144, pp.260-266, 2010.

6. X. Tang, K. Remmel, X. Lan, J. Deng, H. Xiao, J. Dong, "Perovskite-Type Oxide Thin Film Integrated Fiber Optic Sensor for High Temperature Hydrogen Measurement," Analytical Chemistry, v.81, pp.7844-7848, 2009.

7. Y. Han, L. Wei, T. Wei, H.-L. Tsai, H. Xiao, "Surface Enhanced Raman Scattering Silica Substrate Fast Fabrication by Femtosecond Laser Pulses,” Applied Physics A, v.97, pp.721-724, 2009.

8. Y. Han, C. Lin, H. Xiao, H.-L. Tsai, "Femtosecond laser-induced silicon surface morphology in water confinement," Microsystem Technologies, v.15, pp.1045-1049, 2009.

9. T. Wei, X. Lan, and H. Xiao, "Fiber Inline Core-Cladding-Mode Mach-Zehnder Interferometer Fabricated by Two-Point CO2 Laser Irradiations," Photonic Technology Letters, v. 21, pp.669$671,2009$.

10. J. Zhang, X. Tang, J. Dong, T. Wei, H. Xiao, “A Zeolite Thin Film-Coated Long Period Fiber Grating Sensor for Measuring Trace Organic Vapors," Sensors \& Actuators: B. Chemical, v.135, pp. 420-425, 2009.

11. Y. Li , T. Wei, J.A. Montoya, S.V. Saini, X. Lan, X. Tang, J. Dong, H. Xiao, "Measurement of CO2 Laser Irradiation Induced Refractive Index Modulation in Singlemode Fiber towards LPFG 
Design and Fabrication,” Applied Optics, v. 47, pp.5296-5304, 2008.

12. J. Zhang, X. Tang, J. Dong, T. Wei, H. Xiao, "Zeolite thin film-coated long period fiber grating sensor for measuring trace chemical," Optics Express, v.16, pp.8317-8323, 2008.

13. T. Wei, Y.K. Han, H.-L. Tsai, H. Xiao, "Miniaturized fiber inline Fabry-Perot interferometer fabricated with a femtosecond laser," Optics Letters, v.33, pp.536-538, 2008. Featured in Photonics Spectra, "Tiny Fiber Fabry-Perot Created with Femtosecond Pulses: Extrinsic interferometer could detect chemicals in harsh environments," by Breck Hitz, May. 2008. Selected for Virtual Journal of Ultrafast Science, v.7, Is.5, May 2008.

14. T. Wei, Y.K. Han, Y.J. Li, H.-L. Tsai, H. Xiao, "Temperature-insensitive miniaturized fiber inline Fabry-Perot interferometer for highly sensitive refractive index measurement," Optics Express, v.16, pp.5764-5769, 2008.

15. X.T. Wei, T. Wei, H. Xiao and Y.S. Lin, "Nano-structured Pd-Long Period Fiber Grating Integrated Optical Sensor for Hydrogen Detection," Sensors \& Actuators: B. Chemical, v.134, pp.687-693, 2008.

16. N. Liu, J. Hui, C. Sun, J. Dong, L. Zhang, and H. Xiao, "Nanoporous Zeolite Thin Film-Based Fiber Intrinsic Fabry-Perot Interferometric Sensor for Detection of Dissolved Organics in Water," Sensors, v.6, pp.835, 2006.

17. J. Zhang, M. Luo, H. Xiao, J. Dong, "Interferometric Study on the Adsorption-Dependent Refractive Index of Silicalite Thin Films Grown on Optical Fibers," Chemistry of Materials, v.18, pp.4-6, 2006.

18. A. Kulkarni, J. Dong, A. Bourandas, P.A. Fuierer, H. Xiao, "Synthesis and Characterization of Nanocrystalline (Zr0.84Y0.16)O1.92-(Ce0.85Sm0.15)O1.925 Heterophase Thin Film,” Journal of Material Research Society, v.21, pp.500-504, 2006.

19. J. Zhang, J. Dong, M. Luo, H. Xiao, S. Murad, R.A. Normann, "Zeolite-fiber integrated optical chemical sensors for detection of dissolved organics in water," Langmuir, v.21, pp. 8609-8612, 2005.

20. H. Xiao, J. Zhang, J. Dong, M. Luo, R. Lee, and V. Romero, "Synthesis of MFI zeolite films on optical fibers for detection of chemical vapors," Optics Letters, v.30, p.1270-1272, 2005. Featured in Photonics Spectra, "Fiber Sensor Provides Real-Time, Continuous Monitoring: Device is effective for both liquid- and gas-phase observations," by Breck Hitz, Dec. 2005. Also featured in the MRS Bulletin, "Zeolite-coated optical fibers demonstrated as sensitive chemical vapor detectors," by C. McCormick, July, 2005. 


\section{Conference Publications}

1. T. Wei, X. Fang and H. Xiao, "Glass/Sapphire Optical Fiber inline Fabry-Perot Interferometer Fabricated using Femtosecond Laser for High Temperature Sensing Applications," Proceeding of 57th Int'1 Instrumentation Symposium, 20-24 June 2011, St. Louis, MO, USA.

2. H. Xiao and T. Wei, "High Temperature Optical Fiber Sensors (Invited)," 57th International Instrumentation Symposium, 20-24 June 2011, St. Louis, MO, USA.

3. X. Lan, B. Cheng, T. Wei, Q. Han, A. Kaur, Y. Zhang, J. Huang, X. Fang, and H. Xiao, "Zeolite thin film coated high order mode long-period fiber grating vapor sensor based on intensity measurement," Proceeding of 57th Int'1 Instrumentation Symposium, 20-24 June 2011, St. Louis, MO, USA.

4. T. Wei, X. Lan, Y. Zhang, H. Duan, H. Xiao, "Optical fiber sensors for high temperature harsh environment applications," SPIE Proceedings, vol. 7647, 2010.

5. H. Xiao, T. Wei, X. Lan, Y. Zhang, H. Duan, and Y. Han, and H-L. Tsai, "Optical Fiber Sensors for Harsh Environment Monitoring," Invited, ASCE Earth \& Space 2010 Conference, Honolulu, Hawaii, March 11-14, 2010.

6. H. Xiao, T. Wei, X. Lan, Y. Zhang, Y. Han, and H.-L. Tsai, "Optical fiber microsensors for high temperature harsh environment applications," Joint PIWG and EVI-GTI Fall Conference, October 26 through October 29, 2010, Newport News, VA.

7. G. Chen, H. Xiao, Y. Huang, Y. Zhang, Z. Zhou, "Simultaneous strain and temperature monitoring of a small-scale steel column in harsh environment with long-period fiber grating sensors," SPIE Proceedings, vol. 7647, 2010.

8. Y. Zhang, Y. Huang, G. Chen, Z. Zhou, H. Xiao, "Simultaneous measurement of strain and temperature using dual long-period fiber gratings with strain compensation," SPIE Proceedings, vol. 7682, 2010.

9. H. Duan, X. Lan, T. Wei, Y. Zhang, H. Xiao, "Characteristics and application of phase-shifted long-period fiber grating fabricated by CO2 laser," SPIE Proceedings, vol. 7682, 2010.

10. Y. Zhang, H. Xiao, T. Wei, X. Lan, H. Duan, "Side-coupled optical fiber devices for sensing applications," SPIE Proceedings, vol. 7682, 2010.

11. H. Xiao, T. Wei, X. Lan, Y. Zhang, "Optical Fiber Harsh Environment Sensors," Invited, the 14th Optoelectronics and Communications Conference (OECC-2009), Hong Kong, July 13-17, 2009.

12. H. Xiao, "Micromachined optical fiber sensors," US-China workshop on optical fiber sensor research, development and applications, Wuhan, China, July 8-10, 2009. 
13. J.A. Drallmeier, U.O. Koylu, J. Sarangapani, H. Xiao, "Implementation of Advanced Fuels and Combustion for Internal Combustion Engines," University of Missouri Energy Summit, Columbia, MO, Apr. 22-23, 2009.

14. H. Xiao, H.L. Tsai, J.A. Drallmeier, "Microphotonic Harsh Environment Sensors for Clean Fuel and Power Generation," University of Missouri Energy Summit, Columbia, MO, Apr. 22-23, 2009.

15. J. Dong, X. Tang, J. Zhang, H. Xiao, "Nanocrystalline Cu-doped zirconia film-coated longperiod fiber grating for CO monitoring at high temperature," Invited, SPIE Proceedings, vol. 7322, 2009.

16. T. Wei, Y. Han, H. Tsai, H. Xiao, "Miniaturized fiber inline Fabry-Perot interferometer for chemical sensing," SPIE Proceedings, vol. 7322, 2009.

17. T. Wei, Y. Li, Y. Zhang, X. Lan, H. Xiao, "Fiber inline core-cladding-mode Michelson interferometer fabricated by one point CO2 laser irradiation," SPIE Proceedings, vol. 7322, 2009.

18. X. Lan, T. Wei, Y. Li, Y. Zhang, X. Tang, J. Dong, H. Xiao, "Study on CO2 laser irradiationinduced mode coupling in a single-mode fiber for development of high-performance miniaturized interferometric sensors," SPIE Proceedings, vol. 7322, 2009.

19. H. Xiao, T. Wei, Xi. Lan, Y. Li, Y. Zhang, "Optical Fiber Harsh Environment Sensors for CoalDerive Energy Generation,” Invited, SPIE-OIT 2008, Beijing, China, Nov. 16-19, 2008.

20. H. Xiao, T. Wei, X.Tang, J. Dong, "Zeolite-Coated Long Period Fiber Grating Sensor for Detection of Trace Chemical Vapors," Invited, SPIE-OIT 2008, Beijing, China, Nov. 16-19, 2008.

21. H. Xiao, T. Wei, J. Montoya, X. Lan, Y. Li, X. Tang, J. Dong, "Miniaturized Chemical Sensors by Functional Integration of Nanomaterials with Highly Sensitive Photonic Devices," Materials Science and Technology 2008 Conference (MS\&T’08), Pittsburgh, PA, USA, Oct. 5-9, 2008.

22. T. Wei, J. Montoya, J. Dong, R. A. Normann, H. Xiao, "Fabrication of Long-Period Fiber Gratings by CO2 Laser Irritations for High Temperature Applications," SPIE Proceedings, vol. 6757, 2007.

23. T. Wei, J. Montoya, H. Xiao, J. Zhang, J. Dong, "Highly sensitive chemical sensors by functional integration of nanoporous zeolites with photonic devices," SPIE Proceedings, vol. 6556, 2006.

24. J. Zhang, T. Wei, H. Xiao, J. Dong, "Zeolite Thin Film-Coated Long-Period Fiber Grating Sensors for Detection of Chemical Vapors with High Sensitivity.” SPIE Proceedings, vol. 6757, 2007.

25. J. Hui, H. Xiao, N. Liu, J. Dong, "Nanoporous Zeolite-Fiber Integrated Microsensors for Highly Sensitive Point Detection of Chemical Agents,” SPIE Proceedings, vol. 6217, 2006. 
26. J. Zhang, N. Liu, J. Hui, H. Xiao, J. Dong, "Zeolite Thin Film-Based Fiber Intrinsic Fabry-Perot Interferometric Sensor for Detection of Dissolved Organics and Ions in Water", AIChE Annual meeting, San Francisco, CA, Nov. 13-17, 2006. 


\section{Supported Graduate Students}

Prof. Hai Xiao (PI)' group at Department of Electrical and Computer Engineering, Missouri University of Science and Technology: Tao Wei, John Montoya, Xinwei Lan, Amardeep Kaur, Hongbiao Duan

Prof. Junhang Dong (Co-PI), Department of Chemical and Materials Engineering University of Cincinnati: Jian Zhang, Xiling Tang, Hongmin Jiang

Prof. Jerry Lin (Co-PI), Department of Chemical and Materials Engineering Arizona State University: Xiaotong Wei 